\title{
Modelos mistos \\ para populações finitas \\ com erros de medida \\ endógenos e exógenos
}

\section{Germán Moreno Arenas}

\author{
Tese apresentada ao \\ Instituto de Matemática e Estatística \\ da Universidade de São Paulo \\ para obtenção do grau de \\ Doutor em Ciências
}

\author{
Área de Concentração: Estatística \\ Orientador: Prof. Dr. Julio da Motta Singer
}

Durante a elaboração deste trabalho o autor recebeu apoio financeiro do CNPq e da Universidad Industrial de Santander, Bucaramanga, Colômbia 


\section{Modelos mistos \\ para populações finitas \\ com erros de medida \\ endógenos e exógenos}

Este exemplar corresponde à redação

final da tese devidamente corrigida

e defendida por Germán Moreno Arenas

e aprovada pela Comissão Julgadora.

Banca Examinadora:

- Prof. Dr. Julio da Motta Singer (orientador) - IME-USP.

- Prof. Dr. Heleno Bolfarine - IME-USP.

- Prof. Dr. Mario de Castro - ICMC-USP.

- Prof. Dr. Marcel de Toledo Vieira - UFJF.

- Prof. Dr. Cristiano Ferraz - UFP. 
Dedico este trabalho às mulheres de minha vida e coração: María Luzdím (minha mãe), Alba Lucía (minha esposa), Laura Catalina e Juliana Lucía (minhas filhas).

Ao Carlos Augusto e Vladimir In Memoriam, meus sobrinhos que foram embora muito cedo. 


\section{Resumo}

Consideramos a predição ótima de valores latentes com base em dados sujeitos a erros de medida endógenos e exógenos, obtidos a partir de uma amostra aleatória de uma população finita. Consideramos o modelo misto para populações finitas (MMPF) com erros de medida exógenos e endógenos usando o enfoque proposto por Stanek III, Singer \& Lencina (2004) e Stanek III \& Singer (2004), e calculamos o melhor preditor linear não enviesado (BLUP) do valor latente da $i$-ésima unidade selecionada na amostra. Quando as variâncias endógenas são heterocedásticas, o preditor obtido sob o MMPF é diferente do preditor obtido sob o modelo misto usual, pois a constante de encolhimento depende da média das variâncias individuais. Utilizamos simulação para comparar o preditor obtido sob o modelo misto usual (utilizado conforme a interpretação usual) com o preditor obtido sob o MMPF, mostrando que apesar do primeiro ser enviesado, ele geralmente apresenta erro quadrático médio (EQM) menor (ou ligeiramente maior) do que aquele obtido sob o MMPF. Adicionalmente, mostramos como utilizar dois pacotes de software estatístico (Proc MIXED do SAS e lme(nlme) do R), construídos sob o modelo misto usual, para ajustar corretamente modelos em situações com erros exógenos e endógenos, heterocedásticos ou homocedásticos. 


\section{Abstract}

We consider optimal estimation and prediction of latent values based on data subject to endogenous and exogenous measurement errors, obtained via simple random sample from a finite population. We consider a finite population mixed model (FPMM) with endogenous and exogenous measurement errors proposed by Stanek III et al. (2004) and Stanek III \& Singer (2004) and obtained the best linear unbiased predictor (BLUP) of the latent value of the $i$-th unit selected in the sample. When the endogenous variances are heteroscedastic, the predictor obtained under the FPMM is different than the predictor obtained with the usual mixed model, because the shrinkage constant depends on the average of the individual variances. We consider simulation studies to compare the predictor obtained under the usual mixed model (used according to the usual interpretation) with the predictor obtained under the FPMM, and show that the former is biased, but usually presents smaller (or slightly larger) mean squared error (MSE) than the predictor obtained under the FPMM. Additionally, we indicate how two commonly used statistical software packages (SAS's Proc MIXED and R's lme(nlme) ) may be employed to fit mixed models in situations with heteroscedastic or homoscedastic exogenous and endogenous errors. 


\section{Agradecimentos}

Gostaria de agradecer:

À família Sobral Singer: Julio, Maria Lucia, Carolina e Alice, pela amizade e carinho oferecido às minhas três mulheres do coração: Alba Lucía, Laura Catalina e Juliana Lucía. Literalmente abriram as portas de sua casa e fizeram mais tranquila e agradável nossa permanência em São Paulo.

Ao meu orientador, mestre e amigo Julio da Motta Singer, pela grande oportunidade de ser seu orientando, pelo apoio, paciência, firmeza, tranquilidade e força transmitida durante a elaboração deste trabalho e pelos domingos de bicicleta, apesar do frio.

À minha esposa Alba Lucía, pela paciência, compreensão, ternura, experiências compartilhadas nesta grande cidade, pelo amor e apoio nos momentos mais difíceis desta etapa. Alba Lucía tu és a pessoa mais importante na minha vida.

Às irmãs Luna Sanchez, por acreditar em mim e confiar seu patrimônio no sucesso de minha comissão inicial de estudos de mestrado.

Aos professores do Departamento de Estatística do IME-USP, em especial aos professores com os quais tive o prazer de manter um contato maior: Julio, Antônio Carlos, Clélia Maria, Nelson, Heleno e Pedro.

Aos meus amigos Ricardo Monturiol e Luis Carlos Oñate, professores da Escuela de Matemáticas da Universidad Industrial de Santander UIS, por acreditar em mim e confiar seu patrimônio no sucesso de minha comissão de estudos de doutorado.

Ao meu amigo e novo irmão Alexandre Patriota, pela ajuda nas disciplinas, pelas discussões sobre meu trabalho, pelo interesse em aprender comigo algo sobre populações finitas, pela motivação e ajuda com minha tese. Certamente algumas partes deste trabalho também são dele; ah e também pelos constantes "vamos no forrô". 
Ao meu amigo e irmão negado Artur Lemonte, pelas sacanagens compartilhadas, pela motivação nos momentos em que estava mais triste e desanimado; ele sempre usou psicologia inversa, por exemplo, quando falava "por acaso tenho escrito help na minha testa", "já terminou seu trabalho de iniciação cientifica" e outras expressões que não podem ser escritas neste documento.

Ao meu amigo Rafael Braz, pela ajuda nas disciplinas e porque precisei de paciência quando falava que era "bonitão", sendo que é o patinho feio dos quatro de nossa turma.

A todos os meus amigos da Pós-Graduação, especialmente aqueles que se desprenderam de seus computadores portáteis por uma semana para fazer o processo de simulação deste trabalho, tenho um carinho especial por aqueles que compartilharam disciplinas comigo, por suportar minhas piadas sem graça, pelos momentos de distração, pelos grandes churrascos que compartilhamos juntos, pelas pizzas e cervejas às sextas-feiras. Sempre os levarei no meu coração; minha casa na Colômbia é sua casa.

Talvez, eu possa servir como testemunho para muitos meninos de favela; sair do "Barrio la Transición" de Bucaramanga, Colômbia para a grande São Paulo, parecia impossível, mas graças ao apoio financeiro dos sistemas educacionais colombiano e brasileiro consegui concretizar este propósito de ser doutor. Por isso, dedico este trabalho a todos os habitantes de favela. Crianças, vocês podem acreditar, podem chegar bem longe, ainda que seja demorado. 


\section{Sumário}

1 Introdução 1

2 Modelos mistos $\quad 10$

2.1 Modelo misto usual . . . . . . . . . . . . . . . . . . . . . . . . . 10

2.1 .1 Notação e terminologia . . . . . . . . . . . . . . . . . 10

2.1.2 BLUP e BLUE no modelo misto usual quando $k=1 \ldots \ldots 11$

2.1.3 BLUP e BLUE no modelo misto usual quando $k \neq 1 \ldots . . \ldots 13$

2.2 Modelos mistos para populações finitas . . . . . . . . . . . . . . . . 13

2.2 .1 Notação e terminologia . . . . . . . . . . . . . . . . 14

2.2.2 Modelos mistos para população finita com erros de medida endógenos e exógenos . . . . . . . . . . . . . . . . . 15

2.2.3 Cálculo do BLUP usando as variáveis $\tilde{\boldsymbol{Y}}$. . . . . . . . . . . . 19

2.2.4 Modelo misto expandido para população finita com erros de medida endógenos e exógenos . . . . . . . . . . . . . . . 23

2.2.5 Cálculo do BLUP usando as variáveis expandidas $\overleftrightarrow{\boldsymbol{Y}} \ldots \ldots$. . . . . 24

2.2.6 Unicidade do BLUP de $Y_{i}=\mu+B_{i}$ usando as variáveis aleatórias $\tilde{\boldsymbol{Y}} 29$

2.3 Discussão . . . . . . . . . . . . . . . . . . . . . . . 33

3 Comparação entre preditores obtidos sob os modelos mistos usual e para populações finitas $\quad 35$

3.1 Exemplo . . . . . . . . . . . . . . . . . . . . . 35

3.2 Simulação para $N=3$ e $n=2 \ldots \ldots \ldots \ldots \ldots$

3.3 Simulação para outros valores de $N$ e $n \ldots \ldots$. . . . . . . . . 43 
4 Aspectos computacionais $\quad \mathbf{5 0}$

4.1 Modelos com erros de medida endógenos apenas . . . . . . . . . . . . 52

4.2 Modelos com erros de medida exógenos apenas . . . . . . . . . . . . . 57

4.3 Modelos com presença simultânea de erros de medida endógenos e exógenos homocedásticos . . . . . . . . . . . . . . . . . . 60

4.4 Modelos com presença simultânea de erros de medida endógenos e exógenos heterocedásticos: análise do exemplo Seasons Study . . . . . . . . . . . . . 64

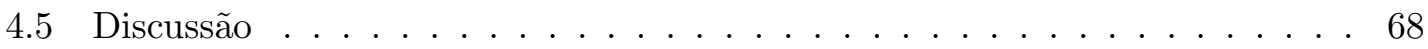

5 Conclusões $\quad 69$

$\begin{array}{ll}\text { A Detalhes sob cálculo dos BLUP } & \mathbf{7 1}\end{array}$

A.1 Valor esperado e variância das variáveis aleatórias $\overleftrightarrow{\boldsymbol{Y}}$. . . . . . . . . . . 71

A.2 Valor esperado e variância das variáveis aleatórias $\tilde{\boldsymbol{Y}}$. . . . . . . . . . . . 73

A.3 Obtenção do BLUP usando as variáveis $\stackrel{\leftrightarrow}{\boldsymbol{Y}} \ldots \ldots$. . . . . . . . . . . . . 74

A.4 Obtenção da variância de $\hat{P}$ usando as variáveis $\tilde{\boldsymbol{Y}}$. . . . . . . . . . . 76

$\begin{array}{ll}\text { B Dados do Nível de Colesterol } & 78\end{array}$

C Códigos R do Capítulo $3 \quad 83$

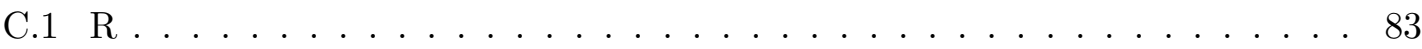

C.2 Resultados da Simulação para $N=3$ e $n=2 \ldots \ldots \ldots$. . . . . 85

$\begin{array}{lll}\text { D Resultados da Simulação para outros valores de } N \text { e } n & 93\end{array}$

E Quocientes EQM $\left[\hat{Q}_{i}^{(1)}\right] / \operatorname{EMQ}\left[\hat{Q}_{i}^{(2)}\right]$ sob erros de medida endógenos com distribuição normal

F Quocientes EQM $\left[\hat{Q}_{i}^{(1)}\right] / \operatorname{EMQ}\left[\hat{Q}_{i}^{(2)}\right]$ sob erros de medida endógenos com distribuição uniforme

G Quocientes EQM $\left[\hat{Q}_{i}^{(1)}\right] / \operatorname{EMQ}\left[\hat{Q}_{i}^{(2)}\right]$ sob erros de medida endógenos com distribuição beta simétrica

H Quocientes EQM $\left[\hat{Q}_{i}^{(1)}\right] / \operatorname{EMQ}\left[\hat{Q}_{i}^{(2)}\right]$ sob erros de medida endógenos com distribuição beta assimétrica 
I Quocientes EQM $\left[\hat{Q}_{i}^{(1)}\right] / \operatorname{EMQ}\left[\hat{Q}_{i}^{(2)}\right]$ sob erros de medida endógenos com distribuição exponencial

J Quocientes $\operatorname{EQM}\left[\hat{Q}_{i}^{(1)}\right] / \operatorname{EMQ}\left[\hat{Q}_{i}^{(2)}\right]$ sob erros de medida endógenos com distribuição gama

K Códigos $\mathrm{R}$ do Capítulo 4

Referências Bibliográficas 


\section{Lista de Tabelas}

1.1 Exemplo de dados do Seasons Study. . . . . . . . . . . . . . . . . . 3

1.2 Valores latentes e variâncias do erro de medida endógeno. . . . . . . . . . . 5

1.3 Variâncias dos erros de medida exógenos induzidas por cada examinador em cada trimestre. . . . . . . . . . . . . . . . . . . . 6

1.4 Variâncias dos erros de medida exógenos. . . . . . . . . . . . . . 7

2.1 Matrizes de definição dos parâmetros ou variáveis de interesse. . . . . . . . 25

3.1 Nível de colesterol, variâncias endógenas, constantes de encolhimento e de ponderação na população $(N=3) \ldots \ldots \ldots \ldots$

3.2 Preditor obtido a partir dos modelos mistos usuais com variâncias hete-

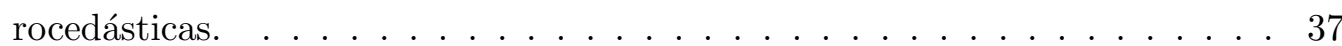

3.3 Preditor obtido a partir dos modelos mistos para populações finitas com variâncias heterocedásticas. . . . . . . . . . . . . . . 38

3.4 Número de populações (num total de 1000) em que o preditor $\hat{Q}_{i}^{(1)}$ tem menor erro quadrático médio que o preditor $\hat{Q}_{i}^{(2)} \ldots \ldots \ldots 40$

3.5 Estatísticas descritivas para os quocientes $\operatorname{EQM}\left[\hat{Q}_{i}^{(1)}\right] / \operatorname{EMQ}\left[\hat{Q}_{i}^{(2)}\right]$ sob erros de medida endógenos com distribuição Normal para $N=3$ e $n=2 \ldots$. . . . 43

3.6 Número de populações em que o preditor $\hat{Q}_{i}^{(1)}$ tem menor erro quadrático médio que o preditor $\hat{Q}_{i}^{(2)}$ de um total de 500 populações simuladas com valores latentes seguindo uma distribuição uniforme. . . . . . . . . . . . . . 46

3.7 Valor máximo do quociente $\operatorname{EQM}\left[\hat{Q}_{i}^{(1)}\right] / \operatorname{EMQ}\left[\hat{Q}_{i}^{(2)}\right]$ com valores latentes seguindo uma distribuição uniforme. . . . . . . . . . . . . . . . . . . 47

4.1 Valores latentes e variâncias endógenas do nível de colesterol. . . . . . . . . 51 
B.1 Dados do nível de colesterol. . . . . . . . . . . . . . . . . . . . . . . . . . . 79

B.2 Continuação: dados do nível de colesterol . . . . . . . . . . . . . . . . . 80

C.1 Estatísticas descritivas para os quocientes $\operatorname{EQM}\left[\hat{Q}_{i}^{(1)}\right] / \operatorname{EMQ}\left[\hat{Q}_{i}^{(2)}\right]$ sob erros de medida endógenos com distribuição Uniforme para $N=3$ e $n=2 \ldots 85$

C.2 Estatísticas descritivas para os quocientes $\operatorname{EQM}\left[\hat{Q}_{i}^{(1)}\right] / \operatorname{EMQ}\left[\hat{Q}_{i}^{(2)}\right]$ sob erros de medida endógenos com distribuição Beta simétrica para $N=3$ e $n=2$. 91

C.3 Estatísticas descritivas para os quocientes $\operatorname{EQM}\left[\hat{Q}_{i}^{(1)}\right] / \operatorname{EMQ}\left[\hat{Q}_{i}^{(2)}\right]$ sob erros de medida endógenos com distribuição Beta assimétrica para $N=3$ e $n=2 \quad 91$

C.4 Estatísticas descritivas para os quocientes $\operatorname{EQM}\left[\hat{Q}_{i}^{(1)}\right] / \operatorname{EMQ}\left[\hat{Q}_{i}^{(2)}\right]$ sob erros de medida endógenos com distribuição Exponencial para $N=3$ e $n=2$. . 91

C.5 Estatísticas descritivas para os quocientes $\operatorname{EQM}\left[\hat{Q}_{i}^{(1)}\right] / \operatorname{EMQ}\left[\hat{Q}_{i}^{(2)}\right]$ sob erros de medida endógenos com distribuição Gama para $N=3$ e $n=2 \ldots 92$

D.1 Número de populações em que o preditor $\hat{Q}_{i}^{(1)}$ tem menor erro quadrático médio que o preditor $\hat{Q}_{i}^{(2)}$ de um total de 500 populações simuladas com valores latentes seguindo uma distribuição assimétrica à esquerda . . . . . . 94

D.2 Número de populações em que o preditor $\hat{Q}_{i}^{(1)}$ tem menor erro quadrático médio que o preditor $\hat{Q}_{i}^{(2)}$ de um total de 500 populações simuladas com valores latentes seguindo uma distribuição assimétrica à direita . . . . . . 95

D.3 Valor máximo do quociente $\operatorname{EQM}\left[\hat{Q}_{i}^{(1)}\right] / \operatorname{EMQ}\left[\hat{Q}_{i}^{(2)}\right]$ com valores latentes seguindo uma distribuição assimétrica à esquerda . . . . . . . . . . . . . . . 96

D.4 Valor máximo do quociente $\operatorname{EQM}\left[\hat{Q}_{i}^{(1)}\right] / \operatorname{EMQ}\left[\hat{Q}_{i}^{(2)}\right]$ com valores latentes seguindo uma distribuição assimétrica à direita . . . . . . . . . . . . . 97 


\section{Lista de Figuras}

1.1 Dados das medições de colesterol do indivíduo número 13. . . . . . . . . . . 4

3.1 Boxplot para quocientes EQM $\left[\hat{Q}_{i}^{(1)}\right] / \operatorname{EMQ}\left[\hat{Q}_{i}^{(2)}\right]$ sob erros de medida endóge-

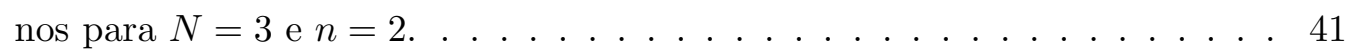

3.2 Quocientes $\operatorname{EQM}\left[\hat{Q}_{i}^{(1)}\right] / \operatorname{EMQ}\left[\hat{Q}_{i}^{(2)}\right]$ sob erros de medida endógenos com distribuição Uniforme para $N=3$ e $n=2 \ldots \ldots$. . . . . . . . . . . 42

3.3 Boxplot para o viés da média populacional quando usamos o preditor $\hat{Q}_{i}^{(1)}$ para $N=3$ e $n=2 \ldots \ldots \ldots \ldots \ldots \ldots \ldots$. . . . . . . . . . . . . . .

B.1 Dados das medições de Colesterol dos indivíduos 1, 2, 3 e 4 . . . . . . . . 81

B.2 Dados das medições de Colesterol dos indivíduos $5,6,7$ e 8 . . . . . . . . 81

B.3 Dados das medições de Colesterol dos indivíduos 9, 10, 11 e 12. . . . . . . . 82

C.1 Quocientes EQM $\left[\hat{Q}_{i}^{(1)}\right] / \operatorname{EMQ}\left[\hat{Q}_{i}^{(2)}\right]$ sob erros de medida endógenos com distribuição Normal para $N=3$ e $n=2 \ldots \ldots$. . . . . . . . 86

C.2 Quocientes $\operatorname{EQM}\left[\hat{Q}_{i}^{(1)}\right] / \operatorname{EMQ}\left[\hat{Q}_{i}^{(2)}\right]$ sob erros de medida endógenos com distribuição Beta simétrica para $N=3$ e $n=2 \ldots \ldots$. . . . . 87

C.3 Quocientes $\operatorname{EQM}\left[\hat{Q}_{i}^{(1)}\right] / \operatorname{EMQ}\left[\hat{Q}_{i}^{(2)}\right]$ sob erros de medida endógenos com distribuição Beta assimétrica para $N=3$ e $n=2 \ldots \ldots$. . . . . 88

C.4 Quocientes $\operatorname{EQM}\left[\hat{Q}_{i}^{(1)}\right] / \operatorname{EMQ}\left[\hat{Q}_{i}^{(2)}\right]$ sob erros de medida endógenos com distribuição Exponencial para $N=3$ e $n=2 \ldots \ldots$. . . . . . . . 89

C.5 Quocientes $\operatorname{EQM}\left[\hat{Q}_{i}^{(1)}\right] / \operatorname{EMQ}\left[\hat{Q}_{i}^{(2)}\right]$ sob erros de medida endógenos com distribuição Gama para $N=3$ e $n=2 \ldots \ldots$. . . . . . . . 90 
E.1 Quocientes EQM $\left[\hat{Q}_{i}^{(1)}\right] / \operatorname{EMQ}\left[\hat{Q}_{i}^{(2)}\right]$ sob erros de medida endógenos com distribuição normal, $N=50$ e $n=5 \ldots \ldots \ldots$. . . . . . . . . 99

E.2 Quocientes $\operatorname{EQM}\left[\hat{Q}_{i}^{(1)}\right] / \operatorname{EMQ}\left[\hat{Q}_{i}^{(2)}\right]$ sob erros de medida endógenos com distribuição normal, $N=50$ e $n=10 \ldots \ldots \ldots$. . . . . . . . . . . . . .

E.3 Quocientes EQM $\left[\hat{Q}_{i}^{(1)}\right] / \operatorname{EMQ}\left[\hat{Q}_{i}^{(2)}\right]$ sob erros de medida endógenos com distribuição normal, $N=50$ e $n=25 \ldots \ldots$. . . . . . . . . . . 101

E.4 Quocientes $\operatorname{EQM}\left[\hat{Q}_{i}^{(1)}\right] / \operatorname{EMQ}\left[\hat{Q}_{i}^{(2)}\right]$ sob erros de medida endógenos com dis-

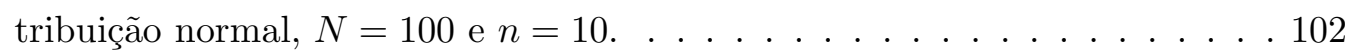

E.5 Quocientes $\operatorname{EQM}\left[\hat{Q}_{i}^{(1)}\right] / \operatorname{EMQ}\left[\hat{Q}_{i}^{(2)}\right]$ sob erros de medida endógenos com distribuição normal, $N=100$ e $n=30 \ldots \ldots$. . . . . . . . . . 103

E.6 Quocientes $\operatorname{EQM}\left[\hat{Q}_{i}^{(1)}\right] / \operatorname{EMQ}\left[\hat{Q}_{i}^{(2)}\right]$ sob erros de medida endógenos com distribuição normal, $N=100$ e $n=50 \ldots \ldots \ldots$. . . . . . . . . . 104

E.7 Quocientes $\operatorname{EQM}\left[\hat{Q}_{i}^{(1)}\right] / \operatorname{EMQ}\left[\hat{Q}_{i}^{(2)}\right]$ sob erros de medida endógenos com distribuição normal, $N=300$ e $n=30 \ldots \ldots \ldots$. . . . . . . . . . . . . .

E.8 Quocientes $\operatorname{EQM}\left[\hat{Q}_{i}^{(1)}\right] / \operatorname{EMQ}\left[\hat{Q}_{i}^{(2)}\right]$ sob erros de medida endógenos com distribuição normal, $N=300$ e $n=50 \ldots \ldots$. . . . . . . . . . 106

E.9 Quocientes EQM $\left[\hat{Q}_{i}^{(1)}\right] / \operatorname{EMQ}\left[\hat{Q}_{i}^{(2)}\right]$ sob erros de medida endógenos com distribuição normal, $N=300$ e $n=100 \ldots \ldots$. . . . . . . . . . 107

F.1 Quocientes EQM $\left[\hat{Q}_{i}^{(1)}\right] / \operatorname{EMQ}\left[\hat{Q}_{i}^{(2)}\right]$ sob erros de medida endógenos com distribuição uniforme, $N=50$ e $n=5 . \quad \ldots \ldots$. . . . . . . . . 109

F.2 Quocientes $\operatorname{EQM}\left[\hat{Q}_{i}^{(1)}\right] / \operatorname{EMQ}\left[\hat{Q}_{i}^{(2)}\right]$ sob erros de medida endógenos com distribuição uniforme, $N=50$ e $n=10 \ldots \ldots \ldots \ldots \ldots$. . . . . . . . . . . .

F.3 Quocientes $\operatorname{EQM}\left[\hat{Q}_{i}^{(1)}\right] / \operatorname{EMQ}\left[\hat{Q}_{i}^{(2)}\right]$ sob erros de medida endógenos com distribuição uniforme, $N=50$ e $n=25 \ldots \ldots \ldots$. . . . . . . . . . . . . .

F.4 Quocientes $\operatorname{EQM}\left[\hat{Q}_{i}^{(1)}\right] / \operatorname{EMQ}\left[\hat{Q}_{i}^{(2)}\right]$ sob erros de medida endógenos com distribuição uniforme, $N=100$ e $n=10 \ldots \ldots$. . . . . . . . . . 112

F.5 Quocientes $\operatorname{EQM}\left[\hat{Q}_{i}^{(1)}\right] / \operatorname{EMQ}\left[\hat{Q}_{i}^{(2)}\right]$ sob erros de medida endógenos com distribuição uniforme, $N=100$ e $n=30 \ldots \ldots \ldots \ldots \ldots \ldots \ldots$

F.6 Quocientes $\operatorname{EQM}\left[\hat{Q}_{i}^{(1)}\right] / \operatorname{EMQ}\left[\hat{Q}_{i}^{(2)}\right]$ sob erros de medida endógenos com distribuição uniforme, $N=100$ e $n=50 \ldots \ldots$. . . . . . . . . . . 114

F.7 Quocientes EQM $\left[\hat{Q}_{i}^{(1)}\right] / \operatorname{EMQ}\left[\hat{Q}_{i}^{(2)}\right]$ sob erros de medida endógenos com dis-

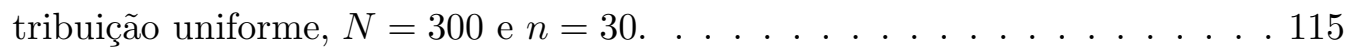


F.8 Quocientes $\operatorname{EQM}\left[\hat{Q}_{i}^{(1)}\right] / \operatorname{EMQ}\left[\hat{Q}_{i}^{(2)}\right]$ sob erros de medida endógenos com distribuição uniforme, $N=300$ e $n=50 \ldots \ldots$. . . . . . . . . . . . . . .

F.9 Quocientes $\operatorname{EQM}\left[\hat{Q}_{i}^{(1)}\right] / \operatorname{EMQ}\left[\hat{Q}_{i}^{(2)}\right]$ sob erros de medida endógenos com distribuição uniforme, $N=300$ e $n=100 \ldots \ldots 117 \ldots \ldots \ldots$

G.1 Quocientes $\operatorname{EQM}\left[\hat{Q}_{i}^{(1)}\right] / \operatorname{EMQ}\left[\hat{Q}_{i}^{(2)}\right]$ sob erros de medida endógenos com distribuição beta simétrica, $N=50$ e $n=5 \ldots$. . . . . . . . . . . . 119

G.2 Quocientes $\operatorname{EQM}\left[\hat{Q}_{i}^{(1)}\right] / \operatorname{EMQ}\left[\hat{Q}_{i}^{(2)}\right]$ sob erros de medida endógenos com distribuição beta simétrica, $N=50$ e $n=10 \ldots \ldots$. . . . . . . . . 120

G.3 Quocientes EQM $\left[\hat{Q}_{i}^{(1)}\right] / \operatorname{EMQ}\left[\hat{Q}_{i}^{(2)}\right]$ sob erros de medida endógenos com distribuição beta simétrica, $N=50$ e $n=25 \ldots \ldots \ldots$. . . . . . . . 121

G.4 Quocientes $\operatorname{EQM}\left[\hat{Q}_{i}^{(1)}\right] / \operatorname{EMQ}\left[\hat{Q}_{i}^{(2)}\right]$ sob erros de medida endógenos com distribuição beta simétrica, $N=100$ e $n=10 \ldots \ldots 122$

G.5 Quocientes EQM $\left[\hat{Q}_{i}^{(1)}\right] / \operatorname{EMQ}\left[\hat{Q}_{i}^{(2)}\right]$ sob erros de medida endógenos com distribuição beta simétrica, $N=100$ e $n=30 \ldots \ldots$. . . . . . . . 123

G.6 Quocientes $\operatorname{EQM}\left[\hat{Q}_{i}^{(1)}\right] / \operatorname{EMQ}\left[\hat{Q}_{i}^{(2)}\right]$ sob erros de medida endógenos com distribuição beta simétrica, $N=100$ e $n=50 \ldots \ldots \ldots$. . . . . . . . . 124

G.7 Quocientes EQM $\left[\hat{Q}_{i}^{(1)}\right] / \operatorname{EMQ}\left[\hat{Q}_{i}^{(2)}\right]$ sob erros de medida endógenos com distribuição beta simétrica, $N=300$ e $n=30$. . . . . . . . . . . . 125

G.8 Quocientes EQM $\left[\hat{Q}_{i}^{(1)}\right] / \operatorname{EMQ}\left[\hat{Q}_{i}^{(2)}\right]$ sob erros de medida endógenos com distribuição beta simétrica, $N=300$ e $n=50 \ldots \ldots$. . . . . . . . 126

G.9 Quocientes $\operatorname{EQM}\left[\hat{Q}_{i}^{(1)}\right] / \operatorname{EMQ}\left[\hat{Q}_{i}^{(2)}\right]$ sob erros de medida endógenos com distribuição beta simétrica, $N=300$ e $n=100$. . . . . . . . . . . . 127

H.1 Quocientes EQM $\left[\hat{Q}_{i}^{(1)}\right] / \operatorname{EMQ}\left[\hat{Q}_{i}^{(2)}\right]$ sob erros de medida endógenos com distribuição beta assimétrica, $N=50$ e $n=5$. . . . . . . . . . . . . . 129

H.2 Quocientes $\operatorname{EQM}\left[\hat{Q}_{i}^{(1)}\right] / \operatorname{EMQ}\left[\hat{Q}_{i}^{(2)}\right]$ sob erros de medida endógenos com distribuição beta assimétrica, $N=50$ e $n=10 \ldots \ldots$. . . . . . . . 130

H.3 Quocientes $\operatorname{EQM}\left[\hat{Q}_{i}^{(1)}\right] / \operatorname{EMQ}\left[\hat{Q}_{i}^{(2)}\right]$ sob erros de medida endógenos com distribuição beta assimétrica, $N=50$ e $n=25 \ldots \ldots$. . . . . . . . . 131

H.4 Quocientes $\operatorname{EQM}\left[\hat{Q}_{i}^{(1)}\right] / \operatorname{EMQ}\left[\hat{Q}_{i}^{(2)}\right]$ sob erros de medida endógenos com distribuição beta assimétrica, $N=100$ e $n=10 \ldots \ldots$. . . . . . . . . 132 
H.5 Quocientes EQM $\left[\hat{Q}_{i}^{(1)}\right] / \operatorname{EMQ}\left[\hat{Q}_{i}^{(2)}\right]$ sob erros de medida endógenos com distribuição beta assimétrica, $N=100$ e $n=30 \ldots \ldots 133$

H.6 Quocientes $\operatorname{EQM}\left[\hat{Q}_{i}^{(1)}\right] / \operatorname{EMQ}\left[\hat{Q}_{i}^{(2)}\right]$ sob erros de medida endógenos com distribuição beta assimétrica, $N=100$ e $n=50 \ldots \ldots \ldots$. . . . . . . 134

H.7 Quocientes EQM $\left[\hat{Q}_{i}^{(1)}\right] / \operatorname{EMQ}\left[\hat{Q}_{i}^{(2)}\right]$ sob erros de medida endógenos com distribuição beta assimétrica, $N=300$ e $n=30 \ldots \ldots 135$

H.8 Quocientes EQM $\left[\hat{Q}_{i}^{(1)}\right] / \operatorname{EMQ}\left[\hat{Q}_{i}^{(2)}\right]$ sob erros de medida endógenos com distribuição beta assimétrica, $N=300$ e $n=50 \ldots \ldots 136$

H.9 Quocientes $\operatorname{EQM}\left[\hat{Q}_{i}^{(1)}\right] / \operatorname{EMQ}\left[\hat{Q}_{i}^{(2)}\right]$ sob erros de medida endógenos com distribuição beta assimétrica, $N=300$ e $n=100 \ldots \ldots$. . . . . . . 137

I.1 Quocientes $\operatorname{EQM}\left[\hat{Q}_{i}^{(1)}\right] / \operatorname{EMQ}\left[\hat{Q}_{i}^{(2)}\right]$ sob erros de medida endógenos com distribuição exponencial, $N=50$ e $n=5 \ldots \ldots \ldots$. . . . . . . . . . . .

I.2 Quocientes $\operatorname{EQM}\left[\hat{Q}_{i}^{(1)}\right] / \operatorname{EMQ}\left[\hat{Q}_{i}^{(2)}\right]$ sob erros de medida endógenos com distribuição exponencial, $N=50$ e $n=10 \ldots \ldots$. . . . . . . . . . 140

I.3 Quocientes $\operatorname{EQM}\left[\hat{Q}_{i}^{(1)}\right] / \operatorname{EMQ}\left[\hat{Q}_{i}^{(2)}\right]$ sob erros de medida endógenos com distribuição exponencial, $N=50$ e $n=25$. . . . . . . . . . . . . 141

I.4 Quocientes EQM $\left[\hat{Q}_{i}^{(1)}\right] / \operatorname{EMQ}\left[\hat{Q}_{i}^{(2)}\right]$ sob erros de medida endógenos com distribuição exponencial, $N=100$ e $n=10 . \ldots \ldots$. . . . . . . . . 142

I.5 Quocientes EQM $\left[\hat{Q}_{i}^{(1)}\right] / \operatorname{EMQ}\left[\hat{Q}_{i}^{(2)}\right]$ sob erros de medida endógenos com distribuição exponencial, $N=100$ e $n=30 \ldots \ldots$. . . . . . . . . 143

I.6 Quocientes $\operatorname{EQM}\left[\hat{Q}_{i}^{(1)}\right] / \operatorname{EMQ}\left[\hat{Q}_{i}^{(2)}\right]$ sob erros de medida endógenos com distribuição exponencial, $N=100$ e $n=50 . \ldots \ldots \ldots$. . . . . . . . 144

I.7 Quocientes $\operatorname{EQM}\left[\hat{Q}_{i}^{(1)}\right] / \operatorname{EMQ}\left[\hat{Q}_{i}^{(2)}\right]$ sob erros de medida endógenos com distribuição exponencial, $N=300$ e $n=30 \ldots \ldots$. . . . . . . . . 145

I.8 Quocientes $\operatorname{EQM}\left[\hat{Q}_{i}^{(1)}\right] / \operatorname{EMQ}\left[\hat{Q}_{i}^{(2)}\right]$ sob erros de medida endógenos com distribuição exponencial, $N=300$ e $n=50 \ldots \ldots$. . . . . . . . . 146

I.9 Quocientes $\operatorname{EQM}\left[\hat{Q}_{i}^{(1)}\right] / \operatorname{EMQ}\left[\hat{Q}_{i}^{(2)}\right]$ sob erros de medida endógenos com distribuição exponencial, $N=300$ e $n=100 \ldots \ldots$. . . . . . . . . . 147

J.1 Quocientes $\operatorname{EQM}\left[\hat{Q}_{i}^{(1)}\right] / \operatorname{EMQ}\left[\hat{Q}_{i}^{(2)}\right]$ sob erros de medida endógenos com distribuição gama, $N=50$ e $n=5 \ldots$. . . . . . . . . . . . . 149 
J.2 Quocientes EQM $\left[\hat{Q}_{i}^{(1)}\right] / \operatorname{EMQ}\left[\hat{Q}_{i}^{(2)}\right]$ sob erros de medida endógenos com distribuição gama, $N=50$ e $n=10 \ldots \ldots$. . . . . . . . . . . . . . . .

J.3 Quocientes $\operatorname{EQM}\left[\hat{Q}_{i}^{(1)}\right] / \operatorname{EMQ}\left[\hat{Q}_{i}^{(2)}\right]$ sob erros de medida endógenos com distribuição gama, $N=50$ e $n=25 \ldots \ldots \ldots \ldots 1 . \ldots \ldots \ldots 1$

J.4 Quocientes EQM $\left[\hat{Q}_{i}^{(1)}\right] / \operatorname{EMQ}\left[\hat{Q}_{i}^{(2)}\right]$ sob erros de medida endógenos com distribuição gama, $N=100$ e $n=10 \ldots \ldots$. . . . . . . . . . . 152

J.5 Quocientes EQM $\left[\hat{Q}_{i}^{(1)}\right] / \operatorname{EMQ}\left[\hat{Q}_{i}^{(2)}\right]$ sob erros de medida endógenos com distribuição gama, $N=100$ e $n=30 \ldots \ldots \ldots$. . . . . . . . . . . . . .

J.6 Quocientes EQM $\left[\hat{Q}_{i}^{(1)}\right] / \operatorname{EMQ}\left[\hat{Q}_{i}^{(2)}\right]$ sob erros de medida endógenos com distribuição gama, $N=100$ e $n=50$. . . . . . . . . . . . . . . . 154

J.7 Quocientes $\operatorname{EQM}\left[\hat{Q}_{i}^{(1)}\right] / \operatorname{EMQ}\left[\hat{Q}_{i}^{(2)}\right]$ sob erros de medida endógenos com distribuição gama, $N=300$ e $n=30$. . . . . . . . . . . . . . . 155

J.8 Quocientes $\operatorname{EQM}\left[\hat{Q}_{i}^{(1)}\right] / \operatorname{EMQ}\left[\hat{Q}_{i}^{(2)}\right]$ sob erros de medida endógenos com dis-

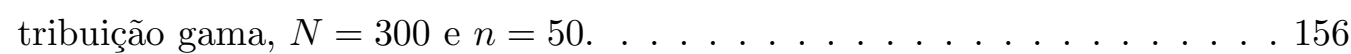

J.9 Quocientes $\operatorname{EQM}\left[\hat{Q}_{i}^{(1)}\right] / \operatorname{EMQ}\left[\hat{Q}_{i}^{(2)}\right]$ sob erros de medida endógenos com distribuição gama, $N=300$ e $n=100 \ldots \ldots \ldots 157 \ldots \ldots \ldots$ 


\section{Capítulo 1}

\section{Introdução}

Predizer o valor latente (valor esperado) de uma ou mais características de unidades amostrais em que são realizadas algumas medições é um dos objetivos da análise estatística. Algumas vezes, essas medições são afetadas por diferentes fontes de variabilidade, gerando erros de medida [Cochran (1977)], também chamados de erros de observação por Sukhatme (1984). Erros de medida podem ser provenientes de duas fontes. A primeira está associada à variabilidade natural da resposta em torno de um valor fixo (valor latente), chamada de variabilidade inerente por Buonaccorsi (2006), ou erro de resposta por Särndal, Swensson \& Wretman (1992). A segunda está associada às condições de medição; e corresponde à variabilidade das medidas em torno do valor latente, gerada, por exemplo, pelos instrumentos de medição ou pela intervenção de examinadores. Para diferenciar claramente os dois tipos de erros de medida, chamaremos o primeiro de erro de medida endógeno e o segundo de erro de medida exógeno.

Erros de medida endógenos podem acontecer mesmo que a medição seja feita com absoluta precisão (erro de medida exógeno nulo). A despesa familiar mensal com alimentos, por exemplo, pode variar de mês a mês em torno de um valor latente, mas pode ser medida sem erro em cada mês. A medição da altura de um indivíduo num determinado instante por diferentes observadores pode servir como exemplo de uma situação onde existe apenas erro de medida exógeno. Medidas do nível de colesterol, por outro lado, variam com o tempo e, além disso, dependem da precisão dos instrumentos e dos produtos químicos usados para produzi-las e, portanto, podem estar sujeitas aos dois tipos de erros simultaneamente. Existem diferentes estratégias para diminuir esses tipos de erros. Em exames médicos, por 
exemplo, protocolos de medição são especificados para diminuir a variabilidade endógena e, além disso, sempre se procura utilizar instrumentos mais precisos e produtos químicos que produzem menores erros de medida exógenos. Nosso interesse é propor métodos estatísticos adequados para analisar dados em que os dois tipos de erros estão presentes.

Como exemplo, podemos considerar um subconjunto de indivíduos da pesquisa Seasons Study [Merriam, Ockene, Hebert, Rosal \& Matthews (1999)], cujo principal objetivo era avaliar padrões sazonais dos níveis de vários tipos de gorduras no corpo humano, como o colesterol total; além disso, pretendia-se estabelecer qual é o impacto da dieta alimentícia, do peso, da exercitação e exposição à luz solar na variação do nível de gorduras. A resposta (nível de colesterol para este trabalho) é obtida mediante uma análise de sangue, preferivelmente depois de 12 horas de jejum para poder reduzir o efeito imediato produzido pelos alimentos. Nesse estudo, o nível de colesterol em cada indivíduo foi medido pelo mesmo examinador três vezes num período curto de tempo (com no máximo duas semanas de diferença). As três medidas foram repetidas quatro vezes em diferentes trimestres, sem ter necessariamente o mesmo examinador do período anterior. Reproduzimos uma parte dos dados na Tabela 1.1. Nas Tabelas B.1 e B.2 do Apêndice B, apresentamos os dados completos para $N=13$ indivíduos, que para efeito ilustrativo, representarão a população alvo neste trabalho.

Se denotamos o nível latente de colesterol para o indivíduo $s$ no dia $d$ por $y_{s d}$, a $r$-ésima medição do nível de colesterol no sangue do indivíduo $s$ no dia $d$ pode ser representada por $Y_{s d r}=y_{s d}+\epsilon_{s d r}$. Se assumimos que $\epsilon_{s d r}$ é uma variável aleatória com valor esperado igual a zero e variância $\sigma_{s d}^{2}$, correspondente à soma da variabilidade natural do colesterol com a variabilidade do erro de medida induzido pela condição de medição (examinador, por exemplo), temos que $y_{s d}$ é o valor esperado do nível de colesterol no dia $d$ para o indivíduo $s$.

O nível latente de colesterol para o indivíduo $s$, denotado por $y_{s}$, é o valor esperado num determinado período de tempo fixo, ${ }^{1} s=1, \ldots, N$. A média populacional dos níveis latentes de colesterol individuais é $\mu=N^{-1} \sum_{s=1}^{N} y_{s}$ e sua variância populacional é $\gamma^{2}=$ $(N-1)^{-1} \sum_{s=1}^{N}\left(y_{s}-\mu\right)^{2}$.

Suponhamos que a variabilidade das três medidas de cada trimestre seja devida ao erro de medida (exógeno) introduzido pelo examinador. Se o indivíduo $s$ foi medido no

\footnotetext{
${ }^{1}$ Podemos definir diferentes períodos de tempo, por exemplo, 365 dias, 12 meses ou 4 trimestres. Por simplicidade, supomos aqui que o valor latente é o valor esperado do nível de colesterol durante 4 trimestres.
} 
Tabela 1.1: Exemplo de dados do Seasons Study.

\begin{tabular}{|c|c|c|c|c|}
\hline Nome & Data & Examinador & Colesterol & Trimestre \\
\hline 1 & $13 / 05 / 1996$ & CS & 180,6 & 1 \\
\hline 1 & $15 / 05 / 1996$ & CS & 206,9 & 1 \\
\hline 1 & $17 / 05 / 1996$ & CS & 234,8 & 1 \\
\hline 1 & $12 / 08 / 1996$ & CS & 171,3 & 2 \\
\hline 1 & $16 / 08 / 1996$ & CS & 174,3 & 2 \\
\hline 1 & $20 / 08 / 1996$ & CS & 185,7 & 2 \\
\hline 1 & 09/11/1996 & $\mathrm{SU}$ & 288,0 & 3 \\
\hline 1 & $13 / 11 / 1996$ & SU & 342,9 & 3 \\
\hline 1 & $17 / 11 / 1996$ & $\mathrm{SU}$ & 278,4 & 3 \\
\hline 1 & $13 / 02 / 1997$ & SU & 276,2 & 4 \\
\hline 1 & $21 / 02 / 1997$ & $\mathrm{SU}$ & 305,3 & 4 \\
\hline 1 & $23 / 02 / 1997$ & $\mathrm{SU}$ & 260,5 & 4 \\
\hline$\vdots$ & $\vdots$ & $\vdots$ & $\vdots$ & $\vdots$ \\
\hline 13 & 01/03/1996 & $\mathrm{SU}$ & 166,1 & 1 \\
\hline 13 & 04/03/1996 & $\mathrm{SU}$ & 118,1 & 1 \\
\hline 13 & 09/03/1996 & $\mathrm{SU}$ & 142,0 & 1 \\
\hline 13 & $20 / 05 / 1996$ & KL & 110,9 & 2 \\
\hline 13 & $21 / 05 / 1996$ & KL & 115,7 & 2 \\
\hline 13 & $24 / 05 / 1996$ & $\mathrm{KL}$ & 99,2 & 2 \\
\hline 13 & $26 / 08 / 1996$ & $\mathrm{KL}$ & 182,0 & 3 \\
\hline 13 & $28 / 08 / 1996$ & KL & 104,5 & 3 \\
\hline 13 & $29 / 08 / 1996$ & $\mathrm{KL}$ & 118,5 & 3 \\
\hline 13 & $22 / 11 / 1996$ & $\mathrm{SU}$ & 107,1 & 4 \\
\hline 13 & $24 / 11 / 1996$ & SU & 192,9 & 4 \\
\hline 13 & $27 / 11 / 1996$ & SU & 89,1 & 4 \\
\hline
\end{tabular}

trimestre $q$ pelo $i$-ésimo examinador, representamos a resposta observada por $y_{s q}+\tilde{W}_{i}$, em que $y_{s q}$ é o nível médio de colesterol para o indivíduo $s$ no trimestre $q$ e $\tilde{W}_{i}$ representa o erro de medida exógeno, com valor esperado igual a zero e variância $\tilde{\sigma}_{i}^{2}$. Para nosso 
exemplo podemos escrever a resposta correspondente como

$$
Y_{s q i}=y_{s q}+\tilde{W}_{i}=y_{s}+W_{s q}+\tilde{W}_{i}=\mu+\beta_{s}+W_{s q}+\tilde{W}_{i},
$$

em que $\beta_{s}=y_{s}-\mu$ e $W_{s q}$ representa o erro de medida endógeno, que assumimos ter valor esperado igual a zero e variância $\sigma_{s}^{2}$.

Na Figura 1.1 apresentamos os dados do indivíduo 13; ele foi medido nos trimestres 1 e 4 pelo examinador $S U$ e nos trimestres 2 e 3 pelo examinador $K L$. Os dados estão representados por "o". O símbolo "+" representa o nível latente de colesterol nesse trimestre. Nas Figuras B.1, B.2 e B.3 do Apêndice B estão representados os dados correspondentes aos outros indivíduos da população alvo. ${ }^{2}$

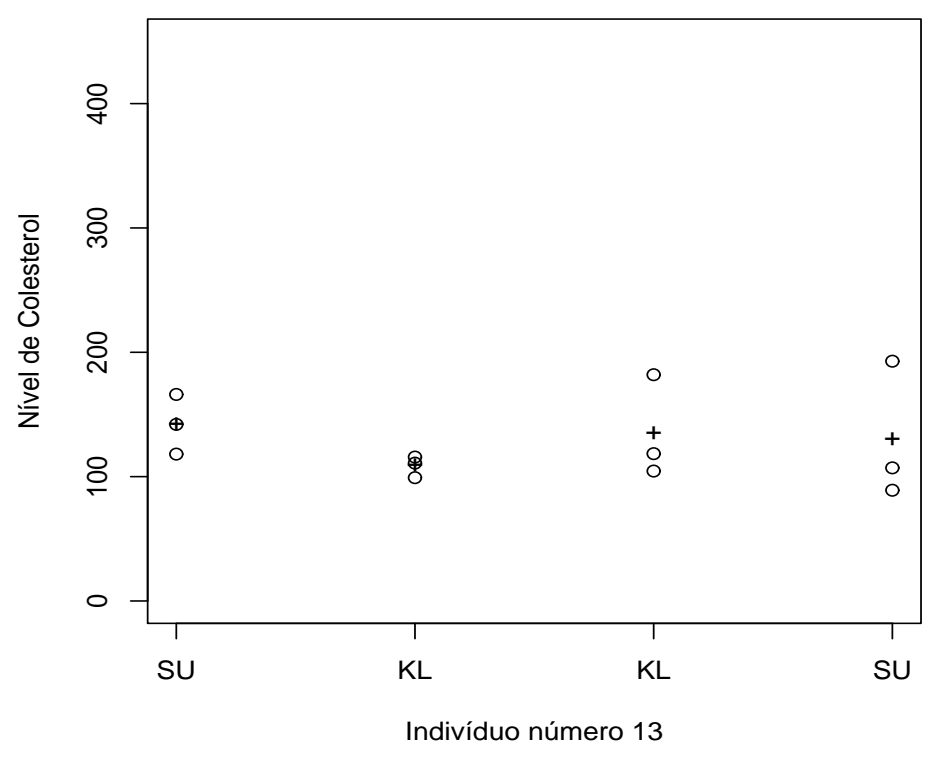

Figura 1.1: Dados das medições de colesterol do indivíduo número 13.

As medidas feitas nos quatro primeiros trimestres contêm a informação para obtermos as variâncias $\gamma^{2}, \sigma_{s}^{2}, s=1, \ldots, 13$ e $\tilde{\sigma}_{i}^{2}, i=1,2,3$. Na Tabela 1.2 apresentamos os valores latentes trimestrais, $y_{s q}, s=1, \ldots, 13, q=1,2,3,4$ (sendo a média das três medidas em cada trimestre) e as variâncias endógenas para cada indivíduo, $\sigma_{s}^{2}=\sum_{q=1}^{4}\left(y_{s q}-y_{s}\right)^{2} / 3$.

\footnotetext{
${ }^{2}$ Para efeito de ilustração, assumimos que os valores representados por "+" são os verdadeiros valores latentes.
} 
Tabela 1.2: Valores latentes e variâncias do erro de medida endógeno.

\begin{tabular}{c|c|c|c|c|c}
\hline $\begin{array}{c}\text { Indivíduo } \\
s\end{array}$ & $\begin{array}{c}\text { Trimestre } 1 \\
y_{s 1}\end{array}$ & $\begin{array}{c}\text { Trimestre } 2 \\
y_{s 2}\end{array}$ & $\begin{array}{c}\text { Trimestre } 3 \\
y_{s}\end{array}$ & $\begin{array}{c}\text { Trimestre } 4 \\
y_{s}\end{array}$ & $\begin{array}{c}\text { Variância } \\
\text { endógena } \sigma_{s}^{2}\end{array}$ \\
\hline 1 & 207,4 & 177,1 & 303,1 & 280,7 & 3545,1 \\
2 & 301,6 & 235,9 & 230,6 & 284,8 & 1250,1 \\
3 & 112,1 & 163,4 & 130,3 & 212,2 & 1932,9 \\
4 & 251,8 & 183,4 & 302,5 & 192,9 & 3083,2 \\
5 & 253,1 & 189,2 & 168,7 & 197,8 & 1299,4 \\
6 & 260,8 & 264,0 & 327,9 & 268,8 & 1013,8 \\
7 & 282,7 & 319,7 & 206,8 & 264,5 & 2216,9 \\
8 & 201,5 & 257,4 & 171,6 & 163,2 & 1815,2 \\
9 & 212,5 & 280,2 & 374,2 & 346,5 & 5222,3 \\
10 & 268,8 & 212,2 & 237,4 & 232,5 & 548,9 \\
11 & 159,3 & 93,2 & 165,9 & 148,7 & 1099,4 \\
12 & 189,4 & 228,2 & 244,1 & 199,4 & 639,1 \\
13 & 142,1 & 108,6 & 135 & 129,7 & 207,8 \\
\hline
\end{tabular}

Na Tabela 1.3 apresentamos as variâncias dos erros de medida exógenos (induzidos pelo examinador) em cada trimestre. As médias dessas variâncias por examinador correspondem às variâncias dos erros de medida exógenos e estão apresentadas na Tabela 1.4 .

Para efeitos de ilustração, tomamos esses valores como as verdadeiras variâncias $\gamma^{2}$, $\sigma_{s}^{2}$ e $\tilde{\sigma}_{i}^{2}$. Nosso interesse está centrado nos seguintes problemas:

i) estimar o nível latente de colesterol para cada indivíduo de uma amostra selecionada aleatoriamente da população sob investigação;

ii) estimar o nível latente de colesterol para os indivíduos que não foram selecionados na amostra;

iii) estimar outras combinações lineares dos níveis latentes de colesterol como a média ou total populacional.

Em geral, centros médicos (clínicas, hospitais, laboratórios, etc.) oferecem somente 
Tabela 1.3: Variâncias dos erros de medida exógenos induzidas por cada examinador em cada trimestre.

\begin{tabular}{|c|c|c|c|c|}
\hline Indivíduo & Trimestre 1 & Trimestre 2 & Trimestre 3 & Trimestre 4 \\
\hline \multirow[t]{2}{*}{1} & 734,6 & 57,7 & 1211,1 & 516,7 \\
\hline & CS & CS & $\mathrm{SU}$ & $\mathrm{SU}$ \\
\hline \multirow[t]{2}{*}{2} & 353,0 & 650,3 & 484,8 & 1220,7 \\
\hline & CS & $\mathrm{KL}$ & $\mathrm{KL}$ & $\mathrm{SU}$ \\
\hline \multirow[t]{2}{*}{3} & 3313,9 & 457,3 & 415,4 & 2151,7 \\
\hline & $\mathrm{KL}$ & $\mathrm{KL}$ & $\mathrm{SU}$ & CS \\
\hline \multirow[t]{2}{*}{4} & 992,6 & 3012,4 & 8527,0 & 2906,8 \\
\hline & CS & CS & $\mathrm{KL}$ & $\mathrm{CS}$ \\
\hline \multirow[t]{2}{*}{5} & 5185,2 & 1220,1 & 169,9 & 374,8 \\
\hline & $\mathrm{KL}$ & KL & $\mathrm{SU}$ & CS \\
\hline \multirow[t]{2}{*}{6} & 238,0 & 1550,4 & 470,1 & 833,7 \\
\hline & $\mathrm{CS}$ & CS & $\mathrm{SU}$ & $\mathrm{SU}$ \\
\hline \multirow[t]{2}{*}{7} & 412,7 & 934,8 & 1071,1 & 995,7 \\
\hline & CS & $\mathrm{CS}$ & CS & $\mathrm{SU}$ \\
\hline \multirow[t]{2}{*}{8} & 3869,1 & 1885,9 & 1596,2 & 250,9 \\
\hline & $\mathrm{SU}$ & $\mathrm{KL}$ & $\mathrm{SU}$ & $\mathrm{SU}$ \\
\hline \multirow[t]{2}{*}{9} & 241,0 & 1668,1 & 2009,1 & 9870,0 \\
\hline & $\mathrm{KL}$ & $\mathrm{KL}$ & $\mathrm{SU}$ & $\mathrm{SU}$ \\
\hline \multirow[t]{2}{*}{10} & 1192,9 & 1246,8 & 132,1 & 474,5 \\
\hline & KL & $\mathrm{SU}$ & CS & $\mathrm{CS}$ \\
\hline \multirow[t]{2}{*}{11} & 552,9 & 65,9 & 93,3 & 1073,9 \\
\hline & $\mathrm{SU}$ & $\mathrm{KL}$ & $\mathrm{SU}$ & $\mathrm{SU}$ \\
\hline \multirow[t]{2}{*}{12} & 1044,2 & 3346,6 & 3004,6 & 1410,7 \\
\hline & $\mathrm{SU}$ & $\mathrm{KL}$ & $\mathrm{SU}$ & $\mathrm{SU}$ \\
\hline \multirow[t]{2}{*}{13} & 576,0 & 72,0 & 1705,8 & 3076,7 \\
\hline & $\mathrm{SU}$ & $\mathrm{KL}$ & $\mathrm{KL}$ & $\mathrm{SU}$ \\
\hline
\end{tabular}


Tabela 1.4: Variâncias dos erros de medida exógenos.

\begin{tabular}{c|c}
\hline Examinador & Variância do erro de medida exógeno \\
\hline $\mathrm{CS}$ & $\tilde{\sigma}_{C S}^{2}=1012,8$ \\
$\mathrm{SU}$ & $\tilde{\sigma}_{S U}^{2}=1623,3$ \\
$\mathrm{KL}$ & $\tilde{\sigma}_{K L}^{2}=2001,1$ \\
\hline
\end{tabular}

a leitura individual, $Y_{s d r}$, como estimador do nível latente de colesterol do indivíduo. Stanek III, Well \& Ockene (1999) compararam o comportamento do melhor estimador linear não enviesado (Best Linear Unbiased Estimator, BLUE) e o melhor preditor linear não enviesado (Best Linear Unbiased Preditor, BLUP) do nível latente de colesterol dos indivíduos selecionados na amostra em diferentes cenários de simulação e mostraram empiricamente que em certos casos é possível incluir informação adicional de outros indivíduos com o intuito de melhorar a estimação. Utilizando a notação semelhante desses autores, a resposta do indivíduo medido no trimestre $q$ pelo $i$-ésimo examinador é expressa como

$$
Y_{q i}=Y_{i}+W_{q i}+\tilde{W}_{i}=\mu+B_{i}+W_{q i}+\tilde{W}_{i}
$$

em que $Y_{i}$ é o valor latente do indivíduo medido pelo $i$-ésimo examinador, $W_{q i}$ representa o erro de medida endógeno do indivíduo medido pelo $i$-ésimo examinador, e $\tilde{W}_{i}$ representa o erro de medida exógeno associado ao $i$-ésimo examinador. O modelo (1.2) é diferente do modelo (1.1), dado que substituimos o índice $s$ que identifica o indivíduo pelo índice $i$ que identifica o examinador e $\beta_{s}$ por $B_{i}$. No modelo (1.1), $\beta_{s}$ é um efeito fixo e no modelo (1.2) $B_{i}$ é um efeito aleatório porque não conhecemos qual unidade amostral é selecionada para ser entrevistada pelo $i$-ésimo examinador. Formalmente, $B_{i}=\sum_{s=1}^{N} U_{i s} \beta_{s}$, em que $U_{i s}$ são variáveis aleatórias indicadoras, que tomam o valor 1 se o indivíduo $s$ é selecionado pelo $i$-ésimo examinador e zero caso contrário.

Os métodos usuais para estimar valores latentes são baseados em modelos lineares mistos (ver Goldberger (1962), Robinson (1991), McCulloch \& Searle (2001), entre outros). Para usar essa metodologia, usamos o modelo (1.2) na forma $Y_{q i}=\mu+B_{i}+E_{q i}$, em que $E_{q i}=W_{q i}+\tilde{W}_{i}$ agrupa os erros de medida, e nesse caso não é possível separar e identificar os dois tipos de erros (exógeno e endógeno).

Stanek III et al. (2004) e Stanek III \& Singer (2004) definiram modelos mistos para populações finitas (MMPF) que permitem distinguir as duas fontes de variabilidade, mas 
não as incluíram simultaneamente. Esses autores mostraram que o melhor preditor linear não enviesado (BLUP) para o valor latente de uma unidade amostral observada consiste da média amostral mais o desvio entre o valor observado na unidade amostral e a média amostral multiplicado por uma constante de encolhimento (shrinkage constant). Quando o erro de medida é associado exclusivamente às condições de medição (erros exógenos), a constante de encolhimento depende das variâncias associadas com cada posição amostral (e.g., com cada examinador). Quando o erro de medida é associado à variabilidade endógena, a constante de encolhimento depende da média populacional das variâncias endógenas das unidades. Nosso alvo é desenvolver um preditor do valor latente individual que leve em conta sua própria variabilidade endógena. Nesse contexto, nossos objetivos especificos são:

1. Descrever a limitação dos modelos mistos usuais quando as respostas incluem erros de medida endógenos e exógenos e apresentar uma solução usando o modelo misto para populações finitas (MMPF) proposto por Stanek III \& Singer (2004).

2. No contexto de amostragem aleatória simples, obter estimadores e/ou preditores para combinações lineares de variáveis aleatórias que definem modelos com erros de medida exógenos e endógenos e discutir suas propriedades.

No Capítulo 2 descrevemos o modelo misto usual e apresentamos a metodologia para a obtenção de preditores ou estimadores ótimos em função de diferentes estruturas de covariância adotadas. Também definimos o modelo misto para populações finitas com presença simultânea de erros de medida endógenos e exógenos, desenvolvemos estimadores/preditores usando uma metodologia equivalente àquela dos modelos mistos usuais e mostramos sua relação com os preditores obtidos a partir de modelos mistos usuais com diferentes estruturas de covariância. Para obter um preditor do valor latente que leve em conta a variabilidade endógena individual, consideramos o modelo misto expandido para populações finitas apresentado por Stanek III et al. (2004), em que tanto os indivíduos quanto sua posição na amostra são representadas. Expressamos os parâmetros ou variáveis aleatórias de interesse como combinações lineares destas novas variáveis, e com base nesse modelo, cujas suposições envolvem apenas aquelas referentes a um processo de amostragem aleatória simples e os primeiros e segundos momentos da distribuição dos erros de medida, obtemos um preditor ótimo para o valor latente de unidades amostrais selecionadas da 
população finita e mostramos suas limitações. No Capítulo 3 apresentamos a comparação do BLUP obtido a partir do modelo misto para população finita com o BLUP obtido a partir do modelo misto usual. Mostramos que mesmo não sendo BLUP, o preditor obtido a partir do modelo misto usual apresenta erro quadrático médio menor do que o BLUP baseado no MMPF.

No Capítulo 4 finalizamos o trabalho mostrando como utilizar dois pacotes de software estatístico (Proc MIXED do $\mathrm{SAS}^{3}$ e lme(nlme) do $\mathrm{R}^{4}$ ), que são construídos sob o modelo misto usual, para ajustar corretamente modelos em situações com erros exógenos ou endógenos heterocedásticos e homocedásticos, erros exógenos e endógenos heterocedásticos e homocedásticos, erros exógenos heterocedásticos e endógenos homocedásticos, erros exógenos homocedásticos e endógenos heterocedásticos. Também apresentamos uma análise dos dados do exemplo Seasons Study e descrevemos alguns resultados e propriedades do preditor obtido a partir do MMPF.

\footnotetext{
${ }^{3}$ www.sas.com

${ }^{4}$ www.r-project.org
} 


\section{Capítulo 2}

\section{Modelos mistos}

\subsection{Modelo misto usual}

Os modelos lineares mistos têm alta relevância em diversas aplicações estatísticas, não só porque constituem alternativas flexíveis para incluir simultaneamente tanto efeitos fixos quanto efeitos aleatórios, mas também porque permitem analisar dados com diferentes estruturas de dependência, dados não balanceados ${ }^{1}$ e dados com distribuições diferentes. Diversas aplicações dos modelos mistos podem ser encontradas em Robinson (1991), Verbeke \& Molenberghs (2000), Diggle, Heagerty, Liang \& Zeger (2002), Demidenko (2004) e Fitzmaurice, Davidian, Verbeke \& Molenberghs (2008), entre outros.

\subsubsection{Notação e terminologia}

Consideramos uma população (universo ou população alvo) finita ou infinita, definida como o conjunto de unidades sobre o qual desejamos obter informação. Uma amostra é um subconjunto de elementos (unidades amostrais) retirados da população cujas características serão medidas e utilizadas para fazer inferências.

Seja $Y_{i k}$ uma variável aleatória correspondente à $k$-ésima medição da resposta de interesse para a $i$-ésima unidade amostral, $i=1, \ldots, n$ e $k=1, \ldots, n_{i}$. O vetor de variáveis aleatórias associadas à $i$-ésima unidade amostral é $\boldsymbol{Y}_{i}=\left(Y_{i 1}, Y_{i 2}, \ldots, Y_{i n_{i}}\right)^{\top}$. O modelo misto usual para $k$-ésima medida da $i$-ésima unidade selecionada na amostra é

$$
Y_{i k}=\mu+B_{i}+E_{i k}, \quad i=1, \ldots, n, \quad k=1, \ldots, n_{i},
$$

\footnotetext{
${ }^{1}$ Quando as unidades amostrais não são medidas necessariamente o mesmo número de vezes.
} 
em que $\mu$ é a resposta média populacional, $B_{i}$ representa o efeito aleatório correspondente à $i$-ésima unidade selecionada, ou seja, é o desvio entre o valor latente da unidade selecionada e $\mu$, e $E_{i k}$ é o erro de medida. Se $W_{i k}$ representa o erro de medida endógeno e $\tilde{W}_{i k}$ o erro de medida exógeno, então $E_{i k}=W_{i k}+\tilde{W}_{i k}$ e esses erros estarão confundidos. Se assumimos que não existe erro exógeno, então $E_{i k}$ representa o erro de medida endógeno. Por outro lado, se assumimos que não existe erro de medida endógeno, então $E_{i k}$ representa o erro de medida exógeno.

Supondo que só temos uma medição para cada unidade amostral ( $k=1$ para todo $i)$, o índice $k$ pode ser eliminado. Em forma matricial podemos escrever o modelo (2.1) como

$$
\boldsymbol{Y}=\mathbf{1}_{n} \mu+\boldsymbol{I}_{n} \boldsymbol{B}+\boldsymbol{E}
$$

em que $\mathbf{1}_{n}$ é um vetor $n$-dimensional com todos os elementos iguais a $1, \boldsymbol{I}_{n}$ é a matriz identidade de ordem $n, \boldsymbol{B}=\left(B_{1}, \ldots, B_{n}\right)^{\top}$, com $\mathbb{E}(\boldsymbol{B})=\mathbf{0}$ e $\mathbb{V}(\boldsymbol{B})=\gamma^{2} \boldsymbol{I}_{n}$, e $\boldsymbol{E}=$ $\left(E_{1}, \ldots, E_{n}\right)^{\top}, \operatorname{com} \mathbb{E}(\boldsymbol{E})=\mathbf{0}$ e $\mathbb{V}(\boldsymbol{E})=\boldsymbol{\Sigma}$.

\subsubsection{BLUP e BLUE no modelo misto usual quando $k=1$}

Seguindo a notação de Robinson (1991) os modelos mistos podem ser expressos na forma

$$
\boldsymbol{Y}=\boldsymbol{X} \boldsymbol{\alpha}+Z \boldsymbol{B}+\boldsymbol{E}
$$

em que $\boldsymbol{Y}$ é um vetor de $n$ variáveis aleatórias observáveis, $\boldsymbol{\alpha}$ é um vetor de $p$ parâmetros desconhecidos representando os efeitos fixos, $\boldsymbol{X}$ e $\boldsymbol{Z}$ são matrizes conhecidas, e $\boldsymbol{B}$ e $\boldsymbol{E}$ são vetores $q$ e $n$-dimensionais, respectivamente, de variáveis aleatórias não observáveis tais que $\mathbb{E}(\boldsymbol{B})=\mathbb{E}(\boldsymbol{E})=\mathbf{0}$,

$$
\mathbb{V}\left(\begin{array}{l}
B \\
E
\end{array}\right)=\left(\begin{array}{ll}
\Gamma & 0 \\
0 & \Sigma
\end{array}\right),
$$

com $\boldsymbol{\Gamma}$ e $\boldsymbol{\Sigma}$ indicando matrizes definidas positivas conhecidas. Para este modelo $\mathbb{E}(\boldsymbol{Y})=$ $\boldsymbol{X} \boldsymbol{\alpha}$ e $\mathbb{V}(\boldsymbol{Y})=\boldsymbol{Z} \boldsymbol{\Gamma} \boldsymbol{Z}^{\top}+\boldsymbol{\Sigma}$. Note que o modelo (2.2) pode ser expresso como (2.3) com $\boldsymbol{X}=\mathbf{1}_{n}, \boldsymbol{\alpha}=\mu$ e $\boldsymbol{Z}=\boldsymbol{I}_{n}$. Consequentemente, $\mathbb{E}(\boldsymbol{Y})=\mathbf{1}_{n} \mu$ e $\mathbb{V}(\boldsymbol{Y})=\gamma^{2} \boldsymbol{I}_{n}+\boldsymbol{\Sigma}$.

O melhor preditor linear não enviesado (BLUP) da variável aleatória $\boldsymbol{B}$ deve ser uma combinação linear dos elementos de $\boldsymbol{Y}$, não enviesado e com o menor erro quadrático médio na classe de estimadores lineares não enviesados. Quando o alvo é o vetor dos efeitos fixos $\boldsymbol{\alpha}$, ele é o melhor estimador linear não enviesado (BLUE). 
Seguindo o desenvolvimento de Goldberger (1962), o BLUE de $\boldsymbol{\alpha}$ e o BLUP de $\boldsymbol{B}$ são, respectivamente, as soluções $\hat{\boldsymbol{\alpha}}$ e $\hat{\boldsymbol{B}}$ para as seguintes equações simultâneas:

$$
\begin{aligned}
\boldsymbol{X}^{\top} \boldsymbol{\Sigma}^{-1} \boldsymbol{X} \hat{\boldsymbol{\alpha}}+\boldsymbol{X}^{\top} \boldsymbol{\Sigma}^{-1} \boldsymbol{Z} \hat{\boldsymbol{B}} & =\boldsymbol{X}^{\top} \boldsymbol{\Sigma}^{-1} \boldsymbol{Y}, \\
\boldsymbol{Z}^{\top} \boldsymbol{\Sigma}^{-1} \boldsymbol{X} \hat{\boldsymbol{\alpha}}+\left(\boldsymbol{Z}^{\top} \boldsymbol{\Sigma}^{-1} \boldsymbol{Z}+\boldsymbol{\Gamma}^{-1}\right) \hat{\boldsymbol{B}} & =\boldsymbol{Z}^{\top} \boldsymbol{\Sigma}^{-1} \boldsymbol{Y},
\end{aligned}
$$

que podem ser expressas como

$$
\left(\begin{array}{cc}
\boldsymbol{X}^{\top} \boldsymbol{\Sigma}^{-1} \boldsymbol{X} & \boldsymbol{X}^{\top} \boldsymbol{\Sigma}^{-1} \boldsymbol{Z} \\
\boldsymbol{Z}^{\top} \boldsymbol{\Sigma}^{-1} \boldsymbol{X} & \boldsymbol{Z}^{\top} \boldsymbol{\Sigma}^{-1} \boldsymbol{Z}+\boldsymbol{\Gamma}^{-1}
\end{array}\right)\left(\begin{array}{c}
\hat{\boldsymbol{\alpha}} \\
\hat{\boldsymbol{B}}
\end{array}\right)=\left(\begin{array}{c}
\boldsymbol{X}^{\top} \boldsymbol{\Sigma}^{-1} \boldsymbol{Y} \\
\boldsymbol{Z}^{\top} \boldsymbol{\Sigma}^{-1} \boldsymbol{Y}
\end{array}\right)
$$

Usando o mesmo processo apresentado em Robinson (1991), podemos mostrar que o BLUP de qualquer combinação linear da forma $\boldsymbol{k}_{1}^{\top} \boldsymbol{\alpha}+\boldsymbol{k}_{2}^{\top} \boldsymbol{B}$, com $\boldsymbol{k}_{1}$ e $\boldsymbol{k}_{2}$ denotando vetores de constantes conhecidas, é $\boldsymbol{k}_{1}^{\top} \hat{\boldsymbol{\alpha}}+\boldsymbol{k}_{2}^{\top} \hat{\boldsymbol{B}}$, em que $\hat{\boldsymbol{\alpha}}$ e $\hat{\boldsymbol{B}}$ são as soluções do sistema de equações (2.6). Por exemplo, suponha que desejamos predizer

$$
Q=\boldsymbol{x}_{0}^{\top} \boldsymbol{\alpha}+\boldsymbol{z}_{0}^{\top} \boldsymbol{B}
$$

em que $\boldsymbol{x}_{0}^{\top}$ e $\boldsymbol{z}_{0}^{\top}$ são vetores de constantes conhecidas. Então, o BLUP de $Q$ deve

- ser linear na amostra, i.e., da forma $P=\boldsymbol{c}^{\top} \boldsymbol{Y}$,

- ser não enviesado, i.e., $\boldsymbol{c}^{\top} \boldsymbol{X} \boldsymbol{\alpha}=\boldsymbol{x}_{0}^{\top} \boldsymbol{\alpha}$,

- ter mínimo erro quadrático médio, i.e., deve ser tal que minimize

$$
\mathbb{V}(P-Q)=\boldsymbol{c}^{\top} \boldsymbol{\Omega} \boldsymbol{c}-2 \boldsymbol{c}^{\top} \boldsymbol{Z} \boldsymbol{\Sigma} \boldsymbol{c}+\boldsymbol{z}_{0}^{\top} \boldsymbol{\Sigma} \boldsymbol{z}_{0}
$$

em que $\boldsymbol{\Omega}=\mathbb{V}(\boldsymbol{Y})$.

Admitindo a existência de todas as matrizes inversas envolvidas (supostamente conhecidas), o resultado da minimização da variância corresponde a

$$
\begin{aligned}
\hat{\boldsymbol{\alpha}} & =\left(\boldsymbol{X}^{\top} \boldsymbol{\Omega}^{-1} \boldsymbol{X}\right)^{-1} \boldsymbol{X}^{\top} \boldsymbol{\Omega}^{-1} \boldsymbol{Y}, \\
\hat{\boldsymbol{B}} & =\boldsymbol{\Gamma} \boldsymbol{Z}^{\top} \boldsymbol{\Omega}^{-1}(\boldsymbol{Y}-\boldsymbol{X} \hat{\boldsymbol{\alpha}}), \\
\hat{Q} & =\boldsymbol{x}_{0}^{\top} \hat{\boldsymbol{\alpha}}+\boldsymbol{z}_{0}^{\top} \hat{\boldsymbol{B}} .
\end{aligned}
$$

Dado o modelo (2.2), suponhamos, por exemplo, que desejamos predizer o nível de colesterol para a $i$-ésima unidade amostral (i.e., para a unidade amostral selecionada na 
$i$-ésima posição na amostra), ou seja com $\boldsymbol{x}_{0}=1$ e $\boldsymbol{z}_{0}=\boldsymbol{e}_{i}$, em que $\boldsymbol{e}_{i}$ é um vetor $n$-dimensional com $i$-ésimo elemento igual a 1 e todos os outros iguais a zero.

Se $\boldsymbol{\Omega}=\gamma^{2} \boldsymbol{I}_{n}+\boldsymbol{\Sigma}$ e $\boldsymbol{\Sigma}=\bigoplus_{i=1}^{n} \sigma_{i}^{2}$, o preditor resultante de $Q_{i}$ é

$$
\hat{Q}_{i}=\hat{\mu}+k_{i}\left(Y_{i}-\hat{\mu}\right),
$$

em que $\hat{\mu}=\hat{\boldsymbol{\alpha}}=\sum_{i=1}^{n} w_{i} Y_{i} / \sum_{i=1}^{n} w_{i}, w_{i}=\left(\gamma^{2}+\sigma_{i}^{2}\right)^{-1}$ e $k_{i}=\gamma^{2} /\left(\gamma^{2}+\sigma_{i}^{2}\right)$. Quando a estrutura de covariância proposta é $\boldsymbol{\Omega}=\gamma^{2} \boldsymbol{I}_{n}+\boldsymbol{\Sigma}$ e $\boldsymbol{\Sigma}=\sigma^{2} \boldsymbol{I}_{n}$, obtemos

$$
\hat{Q}_{i}=\bar{Y}+k\left(Y_{i}-\bar{Y}\right)
$$

em que $\hat{\mu}=\hat{\boldsymbol{\alpha}}=\bar{Y}=\sum_{i=1}^{n} Y_{i} / n$ e $k=\gamma^{2} /\left(\gamma^{2}+\sigma^{2}\right)$.

\subsubsection{BLUP e BLUE no modelo misto usual quando $k \neq 1$}

Para medições desbalanceadas, i.e. com $n_{i}$ medições na $i$-ésima unidade amostral, $i=1, \ldots, n, \operatorname{com} \boldsymbol{\Gamma}=\gamma^{2} \boldsymbol{I}_{n}, \boldsymbol{\Omega}=\gamma^{2} \bigoplus_{i=1}^{n}\left(\mathbf{1}_{n_{i}} \mathbf{1}_{n_{i}}^{\top}\right)+\bigoplus_{i=1}^{n} \sigma_{i}^{2} \boldsymbol{I}_{n_{i}}$, o preditor resultante é

$$
\hat{Q}_{i}=\hat{\mu}+k_{i}\left(\bar{Y}_{i}-\hat{\mu}\right), \quad \bar{Y}_{i}=\sum_{j=1}^{n_{i}} Y_{i j} / n_{i},
$$

em que $\hat{\mu}=\sum_{i=1}^{n} w_{i} \bar{Y}_{i} / \sum_{i=1}^{n} w_{i}, w_{i}=\left(\gamma^{2}+\sigma_{i}^{2} / n_{i}\right)^{-1}$ e $k_{i}=\gamma^{2} /\left(\gamma^{2}+\sigma_{i}^{2} / n_{i}\right)$. Quando a estrutura de covariância é $\boldsymbol{\Omega}=\gamma^{2} \bigoplus_{i=1}^{n}\left(\mathbf{1}_{n_{i}} \mathbf{1}_{n_{i}}^{\top}\right)+\sigma^{2} \boldsymbol{I}_{r}$, em que $M=\sum_{i=1}^{n} n_{i}$, obtemos

$$
\hat{Q}_{i}=\bar{Y}+k w_{i}\left(\bar{Y}_{i}-\bar{Y}\right) \text {, }
$$

em que $\bar{Y}=\sum_{i=1}^{n} w_{i} \bar{Y}_{i} / \sum_{i=1}^{n} w_{i}, \quad w_{i}=\left(1+1 / n_{i}\right)^{-1}$ e $k=\gamma^{2} /\left(\gamma^{2}+\sigma^{2}\right)$. Assim, os resultados da Seção 2.1.2 são caso particular quando $n_{i}=1, \forall i$.

$\mathrm{Na}$ próxima seção mostramos a relação que essas estruturas de covariância têm com os modelos mistos para populações finitas.

\subsection{Modelos mistos para populações finitas}

Nos modelos mistos usuais é possível calcular o BLUE de $\mu$ e o BLUP de $\mu+B_{i}$, o valor latente da $i$-ésima unidade selecionada na amostra. Sob esse enfoque, as unidades não são identificáveis, e consequentemente não é possível estimar o valor $y_{s}$, isto é, o valor latente da unidade com etiqueta $s$ na população. Além disso, as estruturas de covariância 
não estão relacionadas com os erros de medida e sua escolha, em geral, não leva em conta nem características físicas do processo de seleção das unidades amostrais nem aspéctos inerentes ao processo de medida.

Para contornar essa deficiência, usamos a proposta de Stanek III et al. (2004) para representar simultaneamente no modelo as etiquetas das unidades populacionais e suas posições na amostra, adicionando também os erros de medida endógeno e exógeno. Para obtenção de preditores e estimadores usamos a estratégia básica de Scott \& Smith (1969), Royall (1970), Bolfarine \& Zacks (1992) e Stanek III et al. (2004), que consiste em separar as variáveis aleatórias em dois conjuntos, um representando a amostra e outro representando as unidades não amostradas, que chamaremos de remanescentes. Com base nessa separação, escrevemos o preditor como combinação linear das variáveis aleatórias amostrais, usamos os métodos padrão de otimização para obter o BLUP de $\mu+B_{i}$ e o BLUE de $y_{s}$ e mostramos sua relação com as estruturas de covariância adotadas nos modelos (2.10) e (2.11).

\subsubsection{Notação e terminologia}

Definimos uma população finita como uma coleção $\mathfrak{S}$ de $N$ elementos distintos (unidades) para as quais algumas características de interesse podem ser observadas. Consideramos que as $N$ unidades da população finita são identificadas por etiquetas $s=1, \ldots, N$. Vamos associar à unidade $s$, o elemento $y_{s}$ do vetor $\boldsymbol{y}=\left(y_{1}, \ldots, y_{N}\right)^{\top}$, além disso, assumimos que ao selecionar a unidade $s$, observamos também o valor da variável de interesse, $y_{s}$, como definido no Capítulo 1. Definimos a amostra $\mathfrak{s}$ como um subconjunto ordenado de $\mathfrak{S}$. O número total de unidades amostrais em $\mathfrak{s}$ será chamado de tamanho amostral e denotado por $n$. As $n$ posições na amostra serão identificadas por $i=1, \ldots, n$.

Para ilustrar estas definições, consideremos uma população $\mathfrak{S}=\{1,2,3,4,5\}$ composta por 5 unidades da qual selecionamos uma amostra aleatória simples de tamanho $n=3$ sem reposição. Uma amostra em que as unidades com etiquetas 1,4 e 5 foram selecionadas, respectivamente, na terceira, segunda e primeira posição na amostra, pode ser representada como $\mathfrak{s}=(5,4,1)^{\top}$. Por exemplo, seja $\boldsymbol{y}=(23,12,17,16,20)^{\top}$ os valores observáveis da característica de interesse, então para a amostra $\mathfrak{s}=(5,4,1)^{\top}$ a informação 
amostral é

$$
\mathfrak{I}=\left(\begin{array}{ll}
5 & 20 \\
4 & 16 \\
1 & 23
\end{array}\right)
$$

que pode ser obtido pelo produto das matrizes

$$
\mathfrak{I}_{(n \times 2)}=\left(\begin{array}{ccccc}
0 & 0 & 0 & 0 & 1 \\
0 & 0 & 0 & 1 & 0 \\
1 & 0 & 0 & 0 & 0
\end{array}\right)\left(\begin{array}{cc}
1 & 23 \\
2 & 12 \\
3 & 17 \\
4 & 16 \\
5 & 20
\end{array}\right)=\mathfrak{u}_{(n \times N)} \boldsymbol{Z}_{(N \times 2)}
$$

com

$$
\boldsymbol{Z}=\left(\begin{array}{ll}
1 & 23 \\
2 & 12 \\
3 & 17 \\
4 & 16 \\
5 & 20
\end{array}\right)
$$

e u é uma realização da matriz aleatória

$$
\mathfrak{U}=\left(\begin{array}{lllll}
U_{11} & U_{12} & U_{13} & U_{14} & U_{15} \\
U_{21} & U_{22} & U_{23} & U_{24} & U_{25} \\
U_{31} & U_{32} & U_{33} & U_{34} & U_{35}
\end{array}\right)_{(n \times N)}
$$

com $U_{i s}$ igual a 1 quando a unidade $s$ está na posição $i$ da amostra, e zero caso contrário. Com essa idéia, descrevemos a seguir o modelo de permutação aleatória e o modelo misto para populações finitas.

\subsubsection{Modelos mistos para população finita com erros de medida endóge- nos e exógenos}

Consideramos permutações aleatórias da população finita e atribuimos a cada permutação a mesma probabilidade. Denotamos por $\boldsymbol{Y}=\left(Y_{1}, Y_{2}, \ldots, Y_{N}\right)^{\top}$, o vetor aleatório dos valores latentes permutados. Associamos os valores $\boldsymbol{y}=\left(y_{1}, \ldots, y_{N}\right)^{\top}$ e $\boldsymbol{Y}$ incluindo variáveis aleatórias indicadoras, $U_{i s}$, que tomam o valor $1 \mathrm{com}$ probabilidade $1 / N$ se a 
unidade $s$ é selecionada na posição $i$ da permutação, e zero caso contrário. Então o modelo de permutação aleatória é definido como

$$
\boldsymbol{Y}=\boldsymbol{U} \boldsymbol{y}, \quad \text { com } \quad \boldsymbol{U}=\left(\begin{array}{cccc}
U_{11} & U_{12} & \ldots & U_{1 N} \\
U_{21} & U_{22} & \ldots & U_{2 N} \\
\vdots & \vdots & \ddots & \vdots \\
U_{N 1} & U_{N 2} & \ldots & U_{N N}
\end{array}\right)_{(N \times N)} \text { i.e., } Y_{i}=\sum_{s=1}^{N} U_{i s} y_{s}
$$

Note que a matriz $\mathfrak{U}$ contêm as primeiras $n$ linhas da matriz $\boldsymbol{U}$, representando exclusivamente as unidades selecionadas e a ordem de seleção, já a matriz $\boldsymbol{U}$ contem a informação de todas as unidades permutadas.

Usando combinações lineares da forma $\boldsymbol{L}^{\top} \boldsymbol{Y}$, podemos definir diferentes parâmetros populacionais ou variáveis aleatórias de interesse. Por exemplo,

i. se $\boldsymbol{L}=N^{-1} \mathbf{1}_{N}$, temos $\boldsymbol{L}^{\top} \boldsymbol{Y}=N^{-1} \sum_{i=1}^{N} Y_{i}=\mu$, a média populacional;

ii. se $\boldsymbol{L}=\mathbf{1}_{N}$, temos $\boldsymbol{L}^{\top} \boldsymbol{Y}=\sum_{i=1}^{N} Y_{i}=\tau$, o total populacional;

iii. se $\boldsymbol{L}=\boldsymbol{e}_{i}, \operatorname{com} \boldsymbol{e}_{i}$ denotando um vetor $N$-dimensional com $i$-ésimo elemento igual a 1 e todos os outros iguais a zero, temos $\boldsymbol{L}^{\top} \boldsymbol{Y}=\boldsymbol{e}_{i}^{\top} \boldsymbol{Y}=Y_{i}=\mu+B_{i}$, a variável aleatória que representa o valor latente da unidade na $i$-ésima posição das unidades permutadas.

Nosso objetivo é obter estimadores de $\mu$ e $T$ e preditores do valor latente da unidade selecionada na $i$-ésima posição da amostra num modelo em que há erro de medida endógeno e exógeno.

O modelo com erros de medida exógeno e endógeno para a resposta na $i$-ésima posição é definido como

$$
\tilde{Y}_{i}=\sum_{s=1}^{N} U_{i s}\left(y_{s}+W_{s}\right)+\tilde{W}_{i},
$$

em que $\tilde{Y}_{i}$ é a resposta observada na $i$-ésima posição, $W_{s}$ representa o erro de medida endógeno associado à unidade $s$ e $\tilde{W}_{i}$ representa o erro de medida exógeno associado à condição de medida $i, i=1, \ldots, n$.

Fazendo $y_{s}=\mu+\beta_{s}$, de forma que $\beta_{s}$ represente o desvio entre o valor latente da 
unidade $s$ nomeadamente $y_{s}$ e $\mu=N^{-1} \sum_{s=1}^{N} y_{s}$ e partindo de (2.18) podemos escrever

$$
\begin{aligned}
\tilde{Y}_{i} & =\sum_{s=1}^{N} U_{i s}\left(\mu+\beta_{s}+W_{s}\right)+\tilde{W}_{i} \\
& =\mu+\sum_{s=1}^{N} U_{i s} \beta_{s}+\sum_{s=1}^{N} U_{i s} W_{s}+\tilde{W}_{i} \\
& =\mu+B_{i}+W_{i}^{*}+\tilde{W}_{i} \\
& =\mu+B_{i}+E_{i},
\end{aligned}
$$

em que $B_{i}=\sum_{s=1}^{N} U_{i s} \beta_{s}, i=1, \ldots, N$, são efeitos aleatórios e correspondem ao desvio do valor latente da unidade que ficou na $i$-ésima posição da permutação e $\mu, W_{i}^{*}=$ $\sum_{s=1}^{N} U_{i s} W_{s}$ é o erro de medida endógeno associado à unidade que ficou na $i$-ésima posição da permutação e $E_{i}=W_{i}^{*}+\tilde{W}_{i}$. Aqui, $E_{i}$ representa a soma dos dois erros. O modelo (2.19) não distingue estes dois erros de medida $\left(W_{i}^{*}\right.$ e $\left.\tilde{W}_{i}\right)$, pois $i$ indexa apenas a posição na amostra. Além disso, note que não é possível obter a expressão (2.18) a partir de (2.19).

Como $\sum_{s=1}^{N} U_{i s}=1$ para todo $i=1, \ldots, N$, e em cada linha da matriz $\boldsymbol{U}$ existe um único valor de $U_{i s}=1$ com todos os outros iguais a zero, segue que $\tilde{W}_{i}=\sum_{s=1}^{N} U_{i s} \tilde{W}_{i}$. Então, definimos o modelo misto para populações finitas (MMPF) com erros de medida endógenos e exógenos como

$$
\tilde{\boldsymbol{Y}}=\left(\begin{array}{c}
\mu+\sum_{s=1}^{N} U_{1 s} \beta_{s}+\sum_{s=1}^{N} U_{1 s}\left(W_{s}+\tilde{W}_{1}\right) \\
\mu+\sum_{s=1}^{N} U_{2 s} \beta_{s}+\sum_{s=1}^{N} U_{2 s}\left(W_{s}+\tilde{W}_{2}\right) \\
\vdots \\
\mu+\sum_{s=1}^{N} U_{N s} \beta_{s}+\sum_{s=1}^{N} U_{N s}\left(W_{s}+\tilde{W}_{N}\right)
\end{array}\right)=\boldsymbol{X} \boldsymbol{\alpha}+\boldsymbol{Z} \boldsymbol{B}+\boldsymbol{E}^{*}
$$

em que $\boldsymbol{X}=\mathbf{1}_{N}, \boldsymbol{\alpha}=\mu, \boldsymbol{Z}=\boldsymbol{I}_{N}, \boldsymbol{B}=\left(B_{1}, \ldots, B_{N}\right)^{\top}$ e $\boldsymbol{E}^{*}=\left(E_{1}^{*}, \ldots, E_{N}^{*}\right)^{\top}$. O modelo (2.20) difere do modelo (2.2), pois aqui representamos todas as unidades da população permutadas, e não apenas as unidades amostradas.

Utilizando $\xi_{1}$ para indexar a esperança com relação à medida de probabilidade induzida pelas permutações aleatórias, equivalente ao processo de amostragem aleatória 
simples, temos

- $\mathbb{E}_{\xi_{1}}\left(U_{i s}\right)=\frac{1}{N}, i=1, \ldots, N$ e $s=1, \ldots, N$,

- $\mathbb{V}_{\xi_{1}}\left(U_{i s}\right)=\frac{1}{N}\left(1-\frac{1}{N}\right), i=1, \ldots, N$ e $s=1, \ldots, N$,

- $\mathbb{C} \mathbb{V}_{\xi_{1}}\left(U_{i s}, U_{i^{*} s^{*}}\right)=-\frac{1}{N^{2}}, i=i^{*}$ e $s \neq s^{*}$,

- $\mathbb{C} \mathbb{V}_{\xi_{1}}\left(U_{i s}, U_{i^{*} s^{*}}\right)=-\frac{1}{N^{2}}, i \neq i^{*}$ e $s=s^{*}$,

- $\mathbb{C} \mathbb{V}_{\xi_{1}}\left(U_{i s}, U_{i^{*} s^{*}}\right)=\frac{1}{N^{2}(N-1)}, i \neq i^{*}$ e $s \neq s^{*}$,

- $\mathbb{E}_{\xi_{1}}\left[\operatorname{vec}\left(\boldsymbol{U}^{\top}\right)\right]=\frac{1}{N}\left(\mathbf{1}_{N} \otimes \mathbf{1}_{N}\right)$,

- $\mathbb{V}_{\xi_{1}}\left[\operatorname{vec}\left(\boldsymbol{U}^{\top}\right)\right]=\frac{1}{N-1} \boldsymbol{P}_{N} \otimes \boldsymbol{P}_{N}$,

em que $\boldsymbol{P}_{N}=\boldsymbol{I}_{N}-\frac{1}{N} \boldsymbol{J}_{N}, \quad \boldsymbol{J}_{N}=\mathbf{1}_{N} \mathbf{1}_{N}^{\top}, \operatorname{vec}(\boldsymbol{A})$ é o vetor formado pelo empilhamento das colunas da matriz $\boldsymbol{A}$ e $\otimes$ denota o produto de Kronecker [ver Harville (1997)].

Utilizando $\xi_{2}$ para indexar esperança, variância e covariância com relação ao erro de medida endógeno e $\xi_{3}$ para esperança, variância e covariância com relação ao erro de medida exógeno, vamos considerar as seguintes suposições:

- $\mathbb{E}_{\xi_{2}}\left(W_{s}\right)=0, s=1, \ldots, N$,

- $\mathbb{V}_{\xi_{2}}\left(W_{s}\right)=\sigma_{s}^{2}, s=1, \ldots, N$,

- $\mathbb{C V}_{\xi_{2}}\left(W_{s}, W_{s^{*}}\right)=0, s=1, \ldots, N$ e $s^{*}=1, \ldots, N$, e $s \neq s^{*}$,

- $\mathbb{E}_{\xi_{3}}\left(\tilde{W}_{i}\right)=0, i=1, \ldots, N$,

- $\mathbb{V}_{\xi_{3}}\left(\tilde{W}_{i}\right)=\tilde{\sigma}_{i}^{2}, i=1, \ldots, N$,

- $\mathbb{C V}_{\xi_{3}}\left(\tilde{W}_{i}, \tilde{W}_{i^{*}}\right)=0, i=1, \ldots, N, i^{*}=1, \ldots, N$, e $i \neq i^{*}$,

- $\mathbb{E}_{\xi_{2} \xi_{3} \mid \xi_{1}}\left(W_{s}+\tilde{W}_{i}\right)=0, s=1, \ldots, N$ e $i=1, \ldots, N$,

- $\mathbb{V}_{\xi_{2} \xi_{3} \mid \xi_{1}}\left(W_{s}+\tilde{W}_{i}\right)=\sigma_{s}^{2}+\tilde{\sigma}_{i}^{2}, s=1, \ldots, N$ e $i=1, \ldots, N$.

Consequentemente,

$$
\mathbb{E}_{\xi_{1} \xi_{2} \xi_{3}}(\tilde{\boldsymbol{Y}})=\mathbf{1}_{N} \mu
$$

$\mathrm{e}$

$$
\mathbb{V}_{\xi_{1} \xi_{2} \xi_{3}}(\tilde{\boldsymbol{Y}})=\gamma^{2} \boldsymbol{P}_{N}+\bar{\sigma}^{2} \boldsymbol{I}_{N}+\bigoplus_{i=1}^{N} \tilde{\sigma}_{i}^{2},
$$

em que $\bar{\sigma}^{2}=\frac{1}{N} \sum_{s=1}^{N} \sigma_{s}^{2}$ e $\gamma^{2}=\frac{1}{N-1} \sum_{s=1}^{N}\left(y_{s}-\mu\right)^{2}$. Veja Apêndice A.2 para detalhes. 


\subsubsection{Cálculo do BLUP usando as variáveis $\tilde{Y}$}

Consideramos a estrategia de Scott \& Smith (1969), Royall (1970), Bolfarine \& Zacks (1992) e Valliant, Dorfman \& Royall (2000) que consiste em particionar o vetor de variáveis aleatórias $\tilde{\boldsymbol{Y}}$ em dois conjuntos. Sem perda de generalidade tomamos os elementos nas primeiras $n$ posições de $\tilde{\boldsymbol{Y}}$ como a amostra e aquelas nas restantes $(N-n)$ posições como a parte não amostrada ou unidades remanescentes, i.e., consideramos o vetor particionado

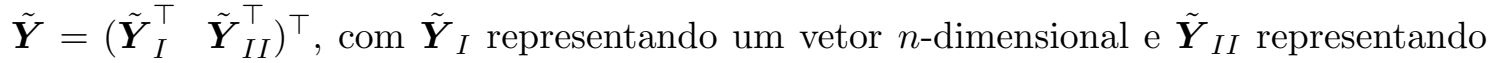
um vetor $(N-n)$-dimensional.

As quantidades de interesse (como aquelas definidas em i, ii e iii) podem ser escritas

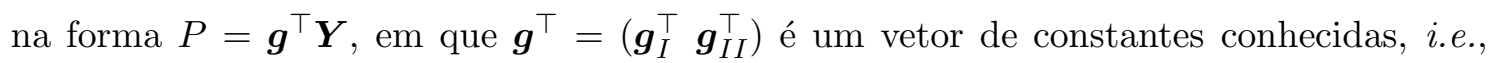
$P=\boldsymbol{g}_{I}^{\top} \boldsymbol{Y}_{I}+\boldsymbol{g}_{I I}^{\top} \boldsymbol{Y}_{I I}$. O BLUP de $P$ deve ser linear na amostra, i.e., $\hat{P}=\left(\boldsymbol{g}_{I}^{\top}+\boldsymbol{a}^{\top}\right) \tilde{\boldsymbol{Y}}_{I}$, não enviesado e minimizar $\mathbb{V}_{\xi_{1} \xi_{2} \xi_{3}}(\hat{P}-P)$. Inicialmente, observemos que

$$
\hat{P}-P=\left(\left(\boldsymbol{g}_{I}^{\top}+\boldsymbol{a}^{\top}\right),-\boldsymbol{g}_{I}^{\top},-\boldsymbol{g}_{I I}^{\top}\right)\left(\begin{array}{c}
\tilde{\boldsymbol{Y}}_{I} \\
\boldsymbol{Y}_{I} \\
\boldsymbol{Y}_{I I}
\end{array}\right)
$$

$\mathrm{e}$

$$
\mathbb{E}_{\xi_{1} \xi_{2} \xi_{3}}(\hat{P}-P)=\left(\left(\boldsymbol{g}_{I}^{\top}+\boldsymbol{a}^{\top}\right),-\boldsymbol{g}_{I}^{\top},-\boldsymbol{g}_{I I}^{\top}\right)\left(\begin{array}{c}
\boldsymbol{X}_{I} \\
\boldsymbol{X}_{I} \\
\boldsymbol{X}_{I I}
\end{array}\right) \mu,
$$

em que $\boldsymbol{X}_{I}=\mathbf{1}_{n}, \boldsymbol{X}_{I I}=\mathbf{1}_{N-n} \mathrm{e}$

$$
\mathbb{V}_{\xi_{1} \xi_{2} \xi_{3}}\left(\begin{array}{c}
\tilde{\boldsymbol{Y}}_{I} \\
\tilde{\boldsymbol{Y}}_{I} \\
\boldsymbol{Y}_{I I}
\end{array}\right)=\left(\begin{array}{ccc}
\tilde{\boldsymbol{V}}_{I} & \boldsymbol{V}_{I} & \boldsymbol{V}_{I, I I} \\
\boldsymbol{V}_{I} & \boldsymbol{V}_{I} & \boldsymbol{V}_{I, I I} \\
\boldsymbol{V}_{I I, I} & \boldsymbol{V}_{I I, I} & \boldsymbol{V}_{I I}
\end{array}\right)
$$

$\operatorname{com}\left(\begin{array}{cc}\boldsymbol{V}_{I} & \boldsymbol{V}_{I, I I} \\ \boldsymbol{V}_{I I, I} & \boldsymbol{V}_{I I}\end{array}\right)=\mathbb{V}_{\xi_{1} \xi_{2} \xi_{3}}\left(\begin{array}{c}\boldsymbol{Y}_{I} \\ \boldsymbol{Y}_{I I}\end{array}\right)$ e $\tilde{\boldsymbol{V}}_{I}=\mathbb{V}_{\xi_{1} \xi_{2} \xi_{3}}\left(\tilde{\boldsymbol{Y}}_{I}\right)$

A restrição de não enviesamento é $\mathbb{E}_{\xi_{1} \xi_{2} \xi_{3}}(\hat{P}-P)=\left(\boldsymbol{a}^{\top}-\boldsymbol{g}_{I I}^{\top}\right)\left(\begin{array}{c}\boldsymbol{X}_{I} \\ \boldsymbol{X}_{I I}\end{array}\right) \mu=0$, e para que esta expressão seja zero para todo $\mu$, devemos ter $\boldsymbol{a}^{\top} \boldsymbol{X}_{I}-\boldsymbol{g}_{I I}^{\top} \boldsymbol{X}_{I I}=0$.

Utilizando multiplicadores de Lagrange, nosso objetivo é minimizar

$$
f(\boldsymbol{a}, \lambda)=\mathbb{V}_{\xi_{1} \xi_{2} \xi_{3}}(\hat{P}-P)+2 \lambda\left(\boldsymbol{a}^{\top} \boldsymbol{X}_{I}-\boldsymbol{g}_{I I}^{\top} \boldsymbol{X}_{I I}\right)
$$


em relação a $\boldsymbol{a}$ e $\lambda$. Diferenciando $f(\boldsymbol{a}, \lambda)$ com relação a $\boldsymbol{a}$ e $\lambda$, e igualando essas derivadas a zero obtemos o BLUP de P, nomeadamente

$$
\hat{P}=\boldsymbol{g}_{I}^{\top}\left[\boldsymbol{X}_{I} \hat{\boldsymbol{\alpha}}+\boldsymbol{V}_{I} \tilde{\boldsymbol{V}}_{I}^{-1}\left(\tilde{\boldsymbol{Y}}_{I}-\boldsymbol{X}_{I} \hat{\boldsymbol{\alpha}}\right)\right]+\boldsymbol{g}_{I I}^{\top}\left[\boldsymbol{X}_{I I} \hat{\boldsymbol{\alpha}}+\boldsymbol{V}_{I I I I} \tilde{\boldsymbol{V}}_{I}^{-1}\left(\tilde{\boldsymbol{Y}}_{I}-\boldsymbol{X}_{I} \hat{\boldsymbol{\alpha}}\right)\right],
$$

em que

$$
\hat{\boldsymbol{\alpha}}=\left(\boldsymbol{X}_{I}^{\top} \tilde{\boldsymbol{V}}_{I}^{-1} \boldsymbol{X}_{I}\right)^{-1} \boldsymbol{X}_{I}^{\top} \tilde{\boldsymbol{V}}_{I}^{-1} \tilde{\boldsymbol{Y}}_{I}
$$

Os detalhes são apresentados no Apêndice A.3.

Como um dos nossos objetivos é estimar o valor latente para a unidade na $i$-ésima posição da amostra, usamos $\boldsymbol{g}^{\top}=\boldsymbol{e}_{i}^{\top}=\left(\begin{array}{ll}\boldsymbol{g}_{I}^{\top} & \boldsymbol{g}_{I I}^{\top}\end{array}\right)^{\top}=\left(\begin{array}{ll}\boldsymbol{e}_{i I}^{\top} & \boldsymbol{e}_{i I I}^{\top}\end{array}\right)^{\top} ; \operatorname{logo}, P_{i}=\boldsymbol{e}_{i I}^{\top} \boldsymbol{Y}_{I}+$ $\boldsymbol{e}_{i I I}^{\top} \boldsymbol{Y}_{I I}$ reduz-se a $Y_{i}=\mu+B_{i}$. Então

$$
\tilde{\boldsymbol{Y}}=\left[\begin{array}{c}
\tilde{\boldsymbol{Y}}_{I} \\
\tilde{\boldsymbol{Y}}_{I I}
\end{array}\right]=\left[\begin{array}{c}
\tilde{Y}_{1} \\
\vdots \\
\tilde{Y}_{n} \\
\tilde{Y}_{n+1} \\
\vdots \\
\tilde{Y}_{N}
\end{array}\right]
$$

com

$$
\mathbb{E}_{\xi_{1} \xi_{2} \xi_{3}}\left[\begin{array}{c}
\tilde{\boldsymbol{Y}}_{I} \\
\tilde{\boldsymbol{Y}}_{I I}
\end{array}\right]=\left[\begin{array}{c}
\mathbf{1}_{n} \\
\mathbf{1}_{N-n}
\end{array}\right] \mu=\left[\begin{array}{c}
\boldsymbol{X}_{I} \\
\boldsymbol{X}_{I I}
\end{array}\right] \mu
$$

$\mathrm{e}$

$$
\mathbb{V}_{\xi_{1} \xi_{2} \xi_{3}}\left[\begin{array}{c}
\tilde{\boldsymbol{Y}}_{I} \\
\tilde{\boldsymbol{Y}}_{I I}
\end{array}\right]=\left[\begin{array}{cc}
\tilde{\boldsymbol{V}}_{I} & \tilde{\boldsymbol{V}}_{I, I I} \\
\tilde{\boldsymbol{V}}_{I I, I} & \tilde{\boldsymbol{V}}_{I I}
\end{array}\right],
$$

em que

$$
\begin{aligned}
{\left[\begin{array}{cc}
\tilde{\boldsymbol{V}}_{I} & \tilde{\boldsymbol{V}}_{I, I I} \\
\tilde{\boldsymbol{V}}_{I I, I} & \tilde{\boldsymbol{V}}_{I I}
\end{array}\right] } & =\gamma^{2}\left[\begin{array}{cc}
\boldsymbol{I}_{n}-\frac{1}{N} \boldsymbol{J}_{n} & -\frac{1}{N} \boldsymbol{J}_{n \times(N-n)} \\
-\frac{1}{N} \boldsymbol{J}_{(N-n) \times n} & \boldsymbol{I}_{N-n}-\frac{1}{N} \boldsymbol{J}_{N-n}
\end{array}\right] \\
& +\bar{\sigma}^{2}\left[\begin{array}{cc}
\boldsymbol{I}_{n} & \mathbf{0}_{n \times(N-n)} \\
\mathbf{0}_{(N-n) \times n} & \boldsymbol{I}_{N-n}
\end{array}\right]+\left[\begin{array}{cc}
\bigoplus_{i=1}^{n} \tilde{\sigma}_{i}^{2} & \mathbf{0}_{n \times(N-n)} \\
\mathbf{0}_{(N-n) \times n} & \bigoplus_{i=n+1}^{N} \tilde{\sigma}_{i}^{2}
\end{array}\right] .
\end{aligned}
$$


Da equação (2.31), $X_{I}=\mathbf{1}_{n}$ e $X_{I I}=\mathbf{1}_{N-n}$. De (2.33), temos

$$
\begin{aligned}
\tilde{V}_{I} & =\gamma^{2}\left[\boldsymbol{I}_{n}-\frac{1}{N} \boldsymbol{J}_{n}\right]+\bar{\sigma}^{2} \boldsymbol{I}_{n}+\bigoplus_{i=1}^{n} \tilde{\sigma}_{i}^{2}, \\
V_{I} & =\gamma^{2}\left[\boldsymbol{I}_{n}-\frac{1}{N} \boldsymbol{J}_{n}\right], \\
V_{I I, I} & =-\frac{\gamma^{2}}{N} \boldsymbol{J}_{(N-n) \times n} .
\end{aligned}
$$

Dado que

$$
\tilde{V}_{I}^{-1}=\bigoplus_{i=1}^{n}\left(\gamma^{2}+\bar{\sigma}^{2}+\tilde{\sigma}_{i}^{2}\right)^{-1}+\frac{\gamma^{2}}{N-\gamma^{2} L} \boldsymbol{m m}^{\top},
$$

em que $L=\sum_{i=1}^{n}\left(\gamma^{2}+\bar{\sigma}^{2}+\tilde{\sigma}_{i}^{2}\right)^{-1}$ e $\boldsymbol{m}$ é um vetor $n$-dimensional com a $i$-ésima componente igual a $\left(\gamma^{2}+\bar{\sigma}^{2}+\tilde{\sigma}_{i}^{2}\right)^{-1}$, então, de $(2.29)$, temos

$$
\hat{\alpha}=\sum_{i=1}^{n} \frac{k_{i} \tilde{Y}_{i}}{\sum_{i=1}^{n} k_{i}}=\overline{\bar{Y}}
$$

com $k_{i}=\left(\gamma^{2}+\bar{\sigma}^{2}+\tilde{\sigma}_{i}^{2}\right)^{-1}$. Consequentemente o BLUP de $P_{i}$ é

$$
\hat{P}_{i}=\left\{\begin{array}{ll}
\overline{\bar{Y}}+\frac{\gamma^{2}}{\gamma^{2}+\bar{\sigma}^{2}+\tilde{\sigma}_{i}^{2}}\left(\tilde{Y}_{i}-\overline{\bar{Y}}\right), & i \leq n \\
\overline{\bar{Y}}, & i>n
\end{array} .\right.
$$

Quando não há nem erros de medida endógenos nem exógenos, $\tilde{\sigma}_{i}^{2}=0, i=1, \ldots, n$, e $\sigma_{s}^{2}=0, s=1, \ldots, N$, temos $\overline{\bar{Y}}=\frac{1}{n} \sum_{i=1}^{n} Y_{i}$, e o BLUP de $P_{i}$ é

$$
\hat{P}_{i}=\left\{\begin{array}{ll}
Y_{i}, & i \leq n \\
\overline{\bar{Y}}, & i>n
\end{array} .\right.
$$

Quando não há variabilidade endógena mas há variabilidade exógena, $\sigma_{s}^{2}=0, s=$ $1, \ldots, N, \bar{\sigma}^{2}=0$. Então $k_{i}=\left(\gamma^{2}+\tilde{\sigma}_{i}^{2}\right)^{-1}$ e o BLUP de $P_{i}$ reduz-se a

$$
\hat{P}_{i}=\left\{\begin{array}{ll}
\overline{\bar{Y}}+\frac{\gamma^{2}}{\gamma^{2}+\tilde{\sigma}_{i}^{2}}\left(\tilde{Y}_{i}-\overline{\bar{Y}}\right), & i \leq n \\
\overline{\bar{Y}}, & i>n
\end{array} .\right.
$$

Quando não há variabilidade exógena mas há variabilidade endógena, $\tilde{\sigma}_{i}^{2}=0, i=$ $1, \ldots, n$, o que implica $k_{1}=\cdots=k_{N}=\gamma^{2}+\bar{\sigma}^{2}$, então $\overline{\bar{Y}}=\frac{1}{n} \sum_{i=1}^{n} \tilde{Y}_{i}$ e o BLUP de $P_{i}$ reduz-se a

$$
\hat{P}_{i}=\left\{\begin{array}{ll}
\overline{\bar{Y}}+\frac{\gamma^{2}}{\gamma^{2}+\bar{\sigma}^{2}}\left(\tilde{Y}_{i}-\overline{\bar{Y}}\right), & i \leq n \\
\overline{\bar{Y}}, & i>n
\end{array} .\right.
$$


As expressões (2.38) e (2.39) são similares apenas no caso homocedástico às expressões (2.10) e (2.11) obtidas sob os modelos mistos usuais com erros heterocedásticos e com erros homocedásticos, respectivamente. A diferença é que aqui as estruturas de covariância estão explicitamente relacionadas com os erros de medida exógenos ou endógenos e a escolha do modelo deve levar em conta as características físicas do processo de medida. Pelos resultados anteriores, o preditor obtido sobre o MMPF com erro de medida exclusivamente exógeno heterocedástico é similar ao preditor obtido sobre os modelos mistos usuais com erros de medida heterocedásticos (2.10). O preditor dos modelos mistos usuais com erros de medida homocedásticos (2.11) com $k=\gamma^{2} /\left(\gamma^{2}+a^{2}\right)$ é similar ao preditor obtido do modelo misto para populações finitas com

- erros de medida endógenos homocedásticos em que $a^{2}=\sigma_{1}^{2}=\cdots=\sigma_{N}^{2}$,

- erros de medida endógenos heterocedásticos em que $a^{2}=\bar{\sigma}^{2}$,

- erros de medida exógenos homocedásticos em que $a^{2}=\tilde{\sigma}_{1}^{2}=\cdots=\tilde{\sigma}_{n}^{2}$.

Quando existe erro de medida exclusivamente endógeno, o termo $E_{i k}$ no modelo misto usual (2.1) representa essa fonte de variabilidade. Supondo que as variâncias endógenas são heterocedásticas o preditor é diferente daquele obtido com o MMPF, pois a constante de encolhimento depende das médias das variâncias individuais. Isso mostra que o BLUP obtido sob o MMPF não envolve a identificabilidade das unidades amostrais. Note que não é possível definir o valor latente para a unidade com etiqueta $j$ na população, $y_{j}=\mu+\beta_{j}$, como combinação linear das variáveis aleatórias $\boldsymbol{Y}$. Além disso, se a unidade com etiqueta $j$ tem variabilidade endógena, seria natural esperar que o preditor de $y_{j}$ dependesse de $\sigma_{j}^{2}$. Para tentar contornar esses problemas vamos considerar um modelo mais geral, com variáveis expandidas. 


\subsubsection{Modelo misto expandido para população finita com erros de me- dida endógenos e exógenos}

Usando a notação similar àquela empregada por Stanek III et al. (2004) definimos um novo conjunto de variáveis aleatórias expandidas $\ddot{\boldsymbol{Y}}=\left(\ddot{\boldsymbol{Y}}_{1}^{\top}, \ldots, \ddot{\boldsymbol{Y}}_{N}^{\top}\right)^{\top}$, com

$$
\ddot{\boldsymbol{Y}}_{i}=\left(\begin{array}{c}
U_{i 1} y_{1} \\
U_{i 2} y_{2} \\
\vdots \\
U_{i N} y_{N}
\end{array}\right) \text { e } \ddot{\boldsymbol{Y}}=v e c\left(\begin{array}{cccc}
U_{11} y_{1} & U_{21} y_{1} & \ldots & U_{N 1} y_{1} \\
U_{12} y_{2} & U_{22} y_{2} & \ldots & U_{N 2} y_{2} \\
\vdots & \vdots & \ddots & \vdots \\
U_{1 N} y_{N} & U_{2 N} y_{N} & \ldots & U_{N N} y_{N}
\end{array}\right)
$$

que também pode ser expresso como

$$
\ddot{\boldsymbol{Y}}=\left(\boldsymbol{I}_{N} \otimes \bigoplus_{s=1}^{N} y_{s}\right) \operatorname{vec}\left(\boldsymbol{U}^{\top}\right) .
$$

Os diferentes parâmetros populacionais ou variáveis aleatórias de interesse podem ser expressos como combinações lineares da forma $\boldsymbol{L}^{\top} \ddot{\boldsymbol{Y}}$. Por exemplo,

i. se $\boldsymbol{L}=N^{-1}\left(\mathbf{1}_{N} \otimes \mathbf{1}_{N}\right)$, temos $\boldsymbol{L}^{\top} \ddot{\boldsymbol{Y}}=N^{-1} \mathbf{1}_{N}^{\top} \boldsymbol{Y}=\mu$,

ii. se $\boldsymbol{L}=\left(\mathbf{1}_{N} \otimes \mathbf{1}_{N}\right)$, temos $\boldsymbol{L}^{\top} \ddot{\boldsymbol{Y}}=\mathbf{1}_{N}^{\top} \boldsymbol{Y}=T$,

iii. se $\boldsymbol{L}=\left(\boldsymbol{e}_{i} \otimes \mathbf{1}_{N}\right)$, temos $\boldsymbol{L}^{\top} \ddot{\boldsymbol{Y}}=\boldsymbol{e}_{i}^{\top} \boldsymbol{Y}=Y_{i}=\mu+B_{i}$,

iv. se $\boldsymbol{L}=\left(\mathbf{1}_{N} \otimes \boldsymbol{e}_{j}\right)$, temos $\boldsymbol{L}^{\top} \ddot{\boldsymbol{Y}}=\mu+\beta_{j}$, isto é, o valor latente para a unidade com etiqueta $j$ na população.

Note que nos casos (i), (ii) e (iii) é possível expressar os parâmetros ou variáveis aleatórias correspondentes usando as variáveis expandidas $\ddot{\boldsymbol{Y}}$ ou as variáveis aleatórias $\boldsymbol{Y}$. No caso (iv), o parâmetro individual $y_{j}$ só pode ser expresso com as variáveis aleatórias expandidas $\ddot{\boldsymbol{Y}}$.

Definimos o modelo misto expandido com erros de medida endógeno e exógeno como

$$
\overleftrightarrow{\boldsymbol{Y}}=\operatorname{vec}\left(\begin{array}{cccc}
U_{11}\left(y_{1}+W_{1}+\tilde{W}_{1}\right) & U_{21}\left(y_{1}+W_{1}+\tilde{W}_{2}\right) & \ldots & U_{N 1}\left(y_{1}+W_{1}+\tilde{W}_{N}\right) \\
U_{12}\left(y_{2}+W_{2}+\tilde{W}_{1}\right) & U_{22}\left(y_{2}+W_{2}+\tilde{W}_{2}\right) & \ldots & U_{N 2}\left(y_{2}+W_{2}+\tilde{W}_{N}\right) \\
\vdots & \vdots & \ddots & \vdots \\
U_{1 N}\left(y_{N}+W_{N}+\tilde{W}_{1}\right) & U_{2 N}\left(y_{N}+W_{N}+\tilde{W}_{2}\right) & \ldots & U_{N N}\left(y_{N}+W_{N}+\tilde{W}_{N}\right)
\end{array}\right)
$$


ou como

$$
\overleftrightarrow{\boldsymbol{Y}}=\left[\boldsymbol{I}_{N} \otimes \bigoplus_{s=1}^{N}\left(y_{s}+W_{s}\right)+\bigoplus_{i=1}^{N} \tilde{W}_{i} \otimes \boldsymbol{I}_{N}\right] \operatorname{vec}\left(\boldsymbol{U}^{\top}\right)=\boldsymbol{X} \mu+\boldsymbol{B}+\boldsymbol{E}^{*}
$$

em que $\boldsymbol{X}=\operatorname{vec}\left(\boldsymbol{U}^{\top}\right), \boldsymbol{B}$ é um vetor $N^{2}$-dimensional com componentes $B_{i s}=U_{i s} \beta_{s}$, $i=1, \ldots, N, \quad s=1, \ldots, N$ e o termo $\boldsymbol{E}^{*}=\left[\boldsymbol{I}_{N} \otimes \bigoplus_{s=1}^{N} W_{s}+\bigoplus_{i=1}^{N} \tilde{W}_{i} \otimes \boldsymbol{I}_{N}\right] \operatorname{vec}\left(\boldsymbol{U}^{\top}\right)$ corresponde à soma dos dois tipos de erros de medida.

Utilizando (2.21) e (2.22), obtemos

$$
\mathbb{E}_{\xi_{1} \xi_{2} \xi_{3}}(\overleftrightarrow{\boldsymbol{Y}})=\left[\boldsymbol{I}_{N} \otimes\left(\bigoplus_{s=1}^{N} y_{s}\right)\right] \frac{1}{N}\left(\mathbf{1}_{N} \otimes \mathbf{1}_{N}\right)=\frac{1}{N}\left(\mathbf{1}_{N} \otimes \boldsymbol{y}\right)
$$

e

$$
\mathbb{V}_{\xi_{1} \xi_{2} \xi_{3}}(\overleftrightarrow{\boldsymbol{Y}})=\boldsymbol{P}_{N} \otimes \Delta+\boldsymbol{I}_{N} \otimes\left(\frac{1}{N} \bigoplus_{s=1}^{N} \sigma_{s}^{2}\right)+\left(\frac{1}{N} \bigoplus_{i=1}^{N} \tilde{\sigma}_{i}^{2}\right) \otimes \boldsymbol{I}_{N}
$$

em que $\Delta=\frac{1}{N-1}\left(\bigoplus_{s=1}^{N} y_{s}\right) \boldsymbol{P}_{N}\left(\bigoplus_{s=1}^{N} y_{s}\right)$. Veja detalhes no apêndice A.1.

As expressões (2.42) e (2.43) envolvem tanto os parâmetros individuais $\left\{y_{1}, y_{2}, \ldots, y_{N}\right\}$ quanto as variâncias endógenas $\left\{\sigma_{1}^{2}, \sigma_{2}^{2}, \ldots, \sigma_{N}^{2}\right\}$ e não simplesmente a média populacional $\mu$ e a média das variâncias dos erros de medida endógenos $\bar{\sigma}^{2}$, como no caso das variáveis $\tilde{Y}$. Isto sugere que o modelo misto expandido contém mais informação e explicita a presença individual das variâncias endógenas. Por outro lado, tanto em (2.24) quanto em (2.43) as variâncias dos erros de medida exógenos, $\tilde{\sigma}_{i}^{2}$, aparecem de forma explicita, indicando que essa fonte de variabilidade é externa dos indivíduos.

\subsubsection{Cálculo do BLUP usando as variáveis expandidas $\stackrel{\leftrightarrow}{Y}$}

De forma similar à $\tilde{\boldsymbol{Y}}$, o vetor $\stackrel{\leftrightarrow}{\boldsymbol{Y}}$ pode ser dividido como $\stackrel{\leftrightarrow}{\boldsymbol{Y}}=\left(\stackrel{\leftrightarrow}{\boldsymbol{Y}}_{I}^{\top} \stackrel{\leftrightarrow}{\boldsymbol{Y}}_{I I}^{\top}\right)^{\top}$, sendo $\stackrel{\leftrightarrow}{\boldsymbol{Y}}_{I}$ um vetor $n N$-dimensional representando a amostra, e $\overleftrightarrow{\boldsymbol{Y}}_{I I}$ um vetor $N(N-n)$-dimensional representando a parte não amostrada. Os parâmetros ou variáveis aleatórias de interesse tem a forma $P=\boldsymbol{g}_{I}^{\top} \ddot{\boldsymbol{Y}}_{I}+\boldsymbol{g}_{I I}^{\top} \ddot{\boldsymbol{Y}}_{I I}$, com os vetores $\boldsymbol{g}_{I}$ e $\boldsymbol{g}_{I I}$ apresentados na Tabela 2.1.

Para preditores lineares da forma $\hat{P}=\left(\boldsymbol{g}_{I}^{\top}+\boldsymbol{a}^{\top}\right) \overleftrightarrow{\boldsymbol{Y}}_{I}$ temos

$$
\hat{P}-P=\left[\left(\boldsymbol{g}_{I}^{\top}+\boldsymbol{a}^{\top}\right),-\boldsymbol{g}_{I}^{\top},-\boldsymbol{g}_{I I}^{\top}\right]\left(\begin{array}{c}
\stackrel{\leftrightarrow}{Y}_{I} \\
\ddot{\boldsymbol{Y}}_{I} \\
\ddot{\boldsymbol{Y}}_{I I}
\end{array}\right),
$$


Tabela 2.1: Matrizes de definição dos parâmetros ou variáveis de interesse.

\begin{tabular}{c|c|c}
\hline Parâmetro/variável & $\boldsymbol{g}_{I}^{\top}$ & $\boldsymbol{g}_{I I}^{\top}$ \\
\hline$T$ & $\left(\mathbf{1}_{n}^{\top} \otimes \mathbf{1}_{N}^{\top}\right)$ & $\left(\mathbf{1}_{N-n}^{\top} \otimes \mathbf{1}_{N}^{\top}\right)$ \\
$\mu$ & $N^{-1}\left(\mathbf{1}_{n}^{\top} \otimes \mathbf{1}_{N}^{\top}\right)$ & $N^{-1}\left(\mathbf{1}_{N-n}^{\top} \otimes \mathbf{1}_{N}^{\top}\right)$ \\
$Y_{i}=\mu+B_{i}$ & $\left(\boldsymbol{e}_{i I}^{\top} \otimes \mathbf{1}_{N}^{\top}\right)$ & $\left(\boldsymbol{e}_{i I I}^{\top} \otimes \mathbf{1}_{N}^{\top}\right)$ \\
$y_{j}=\mu+\beta_{j}$ & $\left(\mathbf{1}_{n}^{\top} \otimes \boldsymbol{e}_{j}^{\top}\right)$ & $\left(\mathbf{1}_{N-n}^{\top} \otimes \boldsymbol{e}_{j}^{\top}\right)$ \\
\hline \multicolumn{1}{c}{} \\
$\mathbb{E}_{\xi_{1} \xi_{2} \xi_{3}}(\hat{P}-P)=\left[\left(\boldsymbol{g}_{I}^{\top}+\boldsymbol{a}^{\top}\right),-\boldsymbol{g}_{I}^{\top},-\boldsymbol{g}_{I I}^{\top}\right]\left(\begin{array}{c}\boldsymbol{X}_{I} \\
\boldsymbol{X}_{I} \\
\boldsymbol{X}_{I I}\end{array}\right) \boldsymbol{y}$,
\end{tabular}

em que $\boldsymbol{X}_{I}=\frac{1}{N}\left(\mathbf{1}_{n} \otimes \boldsymbol{I}_{N}\right), \boldsymbol{X}_{I I}=\frac{1}{N}\left(\mathbf{1}_{N-n} \otimes \boldsymbol{I}_{N}\right)$ e

$$
\mathbb{V}_{\xi_{1} \xi_{2} \xi_{3}}\left(\begin{array}{c}
\overleftrightarrow{\boldsymbol{Y}}_{I} \\
\ddot{\boldsymbol{Y}}_{I} \\
\ddot{\boldsymbol{Y}}_{I I}
\end{array}\right)=\left(\begin{array}{ccc}
\overleftrightarrow{\boldsymbol{V}}_{I} & \ddot{\boldsymbol{V}}_{I} & \ddot{\boldsymbol{V}}_{I, I I} \\
\ddot{\boldsymbol{V}}_{I} & \ddot{\boldsymbol{V}}_{I} & \ddot{\boldsymbol{V}}_{I, I I} \\
\ddot{\boldsymbol{V}}_{I I, I} & \ddot{\boldsymbol{V}}_{I I, I} & \ddot{\boldsymbol{V}}_{I I}
\end{array}\right)
$$

em que

$$
\left(\begin{array}{cc}
\ddot{\boldsymbol{V}}_{I} & \ddot{\boldsymbol{V}}_{I, I I} \\
\ddot{\boldsymbol{V}}_{I I, I} & \ddot{\boldsymbol{V}}_{I I}
\end{array}\right)=\mathbb{V}_{\xi_{1} \xi_{2} \xi_{3}}\left(\begin{array}{c}
\ddot{\boldsymbol{Y}}_{I} \\
\ddot{\boldsymbol{Y}}_{I I}
\end{array}\right)
$$

$\mathrm{e} \stackrel{\leftrightarrow}{\boldsymbol{V}}_{I}=\mathbb{V}_{\xi_{1} \xi_{2} \xi_{3}}\left(\overleftrightarrow{\boldsymbol{Y}}_{I}\right)$

A restrição de não enviesamento é $\mathbb{E}_{\xi_{1} \xi_{2} \xi_{3}}(\hat{P}-P)=\left(\begin{array}{ll}\boldsymbol{a}^{\top} & -\boldsymbol{g}_{I I}^{\top}\end{array}\right)\left(\begin{array}{c}\boldsymbol{X}_{I} \\ \boldsymbol{X}_{I I}\end{array}\right) \boldsymbol{y}=0$ e para que esta expressão seja zero para todo $\boldsymbol{y}$, devemos ter $\boldsymbol{a}^{\top} \boldsymbol{X}_{I}-\boldsymbol{g}_{I I}^{\top} \boldsymbol{X}_{I I}=0$.

Utilizando multiplicadores de Lagrange, o BLUP pode ser obtido por meio da minimização de $\mathbb{V}_{\xi_{1} \xi_{2} \xi_{3}}(\hat{P}-P)$ e é dado por

$$
\hat{P}=\boldsymbol{g}_{I}^{\top}\left[\boldsymbol{X}_{I} \hat{\boldsymbol{\alpha}}+\ddot{\boldsymbol{V}}_{I} \stackrel{\leftrightarrow}{\boldsymbol{V}}_{I}^{-1}\left(\overleftrightarrow{\boldsymbol{Y}}_{I}-\boldsymbol{X}_{I} \hat{\boldsymbol{\alpha}}\right)\right]+\boldsymbol{g}_{I I}^{\top}\left[\boldsymbol{X}_{I I} \hat{\boldsymbol{\alpha}}+\ddot{\boldsymbol{V}}_{I I, I} \stackrel{\leftrightarrow}{\boldsymbol{V}}_{I}^{-1}\left(\overleftrightarrow{\boldsymbol{Y}}_{I}-\boldsymbol{X}_{I} \hat{\boldsymbol{\alpha}}\right)\right]
$$

em que $\hat{\boldsymbol{\alpha}}=\left(\boldsymbol{X}_{I}^{\top} \stackrel{\leftrightarrow}{\boldsymbol{V}}_{I}^{-1} \boldsymbol{X}_{I}\right)^{-1} \boldsymbol{X}_{I}^{\top} \stackrel{\leftrightarrow}{\boldsymbol{V}}_{I}^{-1} \overleftrightarrow{\boldsymbol{Y}}_{I}$. Os detalhes são apresentados no Apêndice A.3.

Para tornar (2.47) operacional precisamos obter a inversa de $\overleftrightarrow{\boldsymbol{V}}_{I}$. De (2.43) temos

$$
\stackrel{\leftrightarrow}{\boldsymbol{V}}_{I}=\left(\boldsymbol{I}_{n}-\frac{1}{N} \boldsymbol{J}_{n}\right) \otimes \Delta+\boldsymbol{I}_{n} \otimes\left(\frac{1}{N} \bigoplus_{s=1}^{N} \sigma_{s}^{2}\right)+\left(\frac{1}{N} \bigoplus_{i=1}^{n} \tilde{\sigma}_{i}^{2}\right) \otimes \boldsymbol{I}_{N}
$$


e para calcular sua inversa vamos inicialmente supor que $\tilde{\sigma}_{i}^{2}=0, i=1, \ldots, n$. Como

$$
\Delta=\frac{1}{N-1}\left(\bigoplus_{s=1}^{N} y_{s}\right)\left(\boldsymbol{I}_{N}-\frac{1}{N} \boldsymbol{J}_{N}\right)\left(\bigoplus_{s=1}^{N} y_{s}\right)=\frac{1}{N-1}\left(\bigoplus_{s=1}^{N} y_{s}^{2}-\frac{1}{N} \boldsymbol{y} \boldsymbol{y}^{\top}\right),
$$

obtemos

$$
\overleftrightarrow{\boldsymbol{V}}_{I}=\frac{1}{N-1}\left(\boldsymbol{I}_{n}-\frac{1}{N} \boldsymbol{J}_{n}\right) \otimes\left(\bigoplus_{s=1}^{N} y_{s}^{2}-\frac{1}{N} \boldsymbol{y} \boldsymbol{y}^{\top}\right)+\boldsymbol{I}_{n} \otimes\left(\frac{1}{N} \bigoplus_{s=1}^{N} \sigma_{s}^{2}\right),
$$

que pode ser escrita como $\stackrel{\leftrightarrow}{V}_{I}=\boldsymbol{I}_{n} \otimes \boldsymbol{A}-\boldsymbol{J}_{n} \otimes \boldsymbol{B}$, em que

$$
\begin{aligned}
\boldsymbol{A} & =\frac{1}{N-1}\left[\bigoplus_{s=1}^{N}\left(y_{s}^{2}+\frac{N-1}{N} \sigma_{s}^{2}\right)-\frac{1}{N} \boldsymbol{y} \boldsymbol{y}^{\top}\right], \\
\boldsymbol{B} & =\frac{1}{N(N-1)}\left[\bigoplus_{s=1}^{N} y_{s}^{2}-\frac{1}{N} \boldsymbol{y} \boldsymbol{y}^{\top}\right],
\end{aligned}
$$

para permitir a utilização dos seguintes teoremas sobre inversa de matrizes, no cálculo de sua inversa.

Teorema 2.2.1. Se existe a inversa da matriz $\boldsymbol{A}$ e de $(n \boldsymbol{B}-\boldsymbol{A})$, e $\boldsymbol{G}=\boldsymbol{I}_{n} \otimes \boldsymbol{A}-\boldsymbol{J}_{n} \otimes \boldsymbol{B}$, então $\boldsymbol{G}^{-1}=\boldsymbol{I}_{n} \otimes \boldsymbol{A}^{-1}-\boldsymbol{J}_{n} \otimes \boldsymbol{A}^{-1} \boldsymbol{B}(n \boldsymbol{B}-\boldsymbol{A})^{-1}$.

Teorema 2.2.2. Seja $\boldsymbol{R}$ uma matriz quadrada de ordem $N \times N, \boldsymbol{s}$ e $\boldsymbol{u}$ vetores $N$ dimensionais; se existe $\boldsymbol{R}^{-1}$, então $\left(\boldsymbol{R}+\boldsymbol{s} \boldsymbol{u}^{\top}\right)^{-1}=\boldsymbol{R}^{-1}-\left(1+\boldsymbol{u}^{\top} \boldsymbol{R}^{-1} \boldsymbol{s}\right)^{-1} \boldsymbol{R}^{-1} \boldsymbol{s} \boldsymbol{u}^{\top} \boldsymbol{R}^{-1}$.

Fazendo $\boldsymbol{R}=\bigoplus_{s=1}^{N}\left(y_{s}^{2}+\frac{N-1}{N} \sigma_{s}^{2}\right), \boldsymbol{s}=-N^{-1} \boldsymbol{y}, \boldsymbol{u}^{\top}=\boldsymbol{y}^{\top}$ e aplicando o Teorema 2.2 .2 , obtemos

$$
\boldsymbol{A}^{-1}=(N-1)\left(\bigoplus_{s=1}^{N} \frac{k_{s}}{y_{s}^{2}}\right)\left[\boldsymbol{I}_{N}+\boldsymbol{y} \boldsymbol{y}^{\top}\left(\bigoplus_{s=1}^{N} \frac{k_{s}}{N(1-\bar{k}) y_{s}^{2}}\right)\right]
$$

em que $k_{s}=y_{s}^{2}\left[y_{s}^{2}+\frac{N-1}{N} \sigma_{s}^{2}\right]^{-1}$. Aplicando novamente o Teorema 2.2.2, obtemos

$$
(n \boldsymbol{B}-\boldsymbol{A})^{-1}=\frac{N(N-1)}{n-N}\left(\bigoplus_{s=1}^{N} \frac{(N-n) k_{s}}{y_{s}^{2}\left(N-n k_{s}\right)}\right)\left[\boldsymbol{I}_{N}+\boldsymbol{y} \boldsymbol{y}^{\top}\left(\bigoplus_{s=1}^{N} \frac{(N-n) k_{s}}{N\left(N-n k_{s}\right) y_{s}^{2} M}\right)\right]
$$

em que $M=\sum_{s=1}^{N}\left\{\left(1-k_{s}\right) /\left(N-n k_{s}\right)\right\}$. 
De (2.43) temos

$$
\begin{aligned}
\ddot{\boldsymbol{V}}_{I} & =\left(\boldsymbol{I}_{n}-\frac{1}{N} \boldsymbol{J}_{n}\right) \otimes \Delta, \\
\ddot{\boldsymbol{V}}_{I I, I} & =-\frac{1}{N} \boldsymbol{J}_{(N-n) \times n} \otimes \Delta .
\end{aligned}
$$

De (2.45) temos $\boldsymbol{X}_{I}^{\top}=\frac{1}{N}\left(\mathbf{1}_{n}^{\top} \otimes \boldsymbol{I}_{N}\right)$, e dado que $\mathbf{1}_{n}^{\top} \boldsymbol{P}_{n}=0$, usamos a expressão alternativa $\stackrel{\leftrightarrow}{\boldsymbol{V}}_{I}^{-1}=\boldsymbol{P}_{n} \otimes \boldsymbol{A}^{-1}+\frac{1}{n} \boldsymbol{J}_{n} \otimes \boldsymbol{A}^{-1}\left[\boldsymbol{I}_{N}-n \boldsymbol{B}(n \boldsymbol{B}-A)^{-1}\right]$, para mostrar que

$$
\begin{aligned}
\boldsymbol{X}_{I}^{\top} \stackrel{\leftrightarrow}{\boldsymbol{V}}_{I}^{-1} \boldsymbol{X}_{I} & =\frac{n}{N^{2}} \boldsymbol{A}^{-1}\left[\boldsymbol{I}_{N}-n \boldsymbol{B}(n \boldsymbol{B}-A)^{-1}\right] \\
& =\frac{n(N-1)}{N^{2}}\left[\bigoplus_{s=1}^{N} \frac{N k_{s}}{y_{s}^{2}\left(N-n k_{s}\right)}+\frac{N-n}{M} \boldsymbol{m m}^{\top}\right]
\end{aligned}
$$

em que $\boldsymbol{m}$ é um vetor $N$-dimensional com $s$-ésima componente igual a $\frac{N}{(N-1)} \frac{y_{s}\left(1-k_{s}\right)}{\sigma_{s}^{2}\left(N-n k_{s}\right)}$, e que

$$
\begin{gathered}
\left(\boldsymbol{X}_{I}^{\top} \stackrel{\leftrightarrow}{\boldsymbol{V}}_{I}^{-1} \boldsymbol{X}_{I}\right)^{-1}=\frac{N^{2}}{n(N-1)}\left[\bigoplus_{s=1}^{N} \frac{y_{s}^{2}\left(N-n k_{s}\right)}{N k_{s}}-\frac{N-n}{N^{2}} \boldsymbol{y} \boldsymbol{y}^{\top}\right] \\
\left(\boldsymbol{X}_{I}^{\top} \overleftrightarrow{\boldsymbol{V}}_{I}^{-1} \boldsymbol{X}_{I}\right)^{-1} \boldsymbol{X}_{I}^{\top} \overleftrightarrow{\boldsymbol{V}}_{I}^{-1}=\frac{N}{n}\left(\mathbf{1}_{n}^{\top} \otimes \boldsymbol{I}_{N}\right) \\
\overleftrightarrow{\boldsymbol{V}}_{I}^{-1}-\left(\boldsymbol{X}_{I}^{\top} \overleftrightarrow{\boldsymbol{V}}_{I}^{-1} \boldsymbol{X}_{I}\right)^{-1} \boldsymbol{X}_{I}^{\top} \overleftrightarrow{\boldsymbol{V}}_{I}^{-1}=(N-1) \boldsymbol{P}_{n} \otimes\left[\bigoplus_{s=1}^{N} \frac{k_{s}}{y_{s}^{2}}+\frac{1}{N(1-\bar{k})} \boldsymbol{r r} \boldsymbol{r}^{\top}\right]
\end{gathered}
$$

em que $\boldsymbol{r}$ é um vetor $N$-dimensional com a $s$-ésima componente igual a $r_{s}=\frac{N}{N-1} \frac{y_{s}\left(1-k_{s}\right)}{\sigma_{s}^{2}}$. Consequentemente de (2.48)-(2.59) o BLUP de $P$ é

$\hat{P}=\left\{\boldsymbol{g}_{I}^{\top}-\frac{N-1}{N} \boldsymbol{g}_{I}^{\top} \boldsymbol{P}_{n}^{\top} \otimes\left[\bigoplus_{s=1}^{N} \frac{\sigma_{s}^{2} k_{s}}{y_{s}^{2}}+\frac{1}{N(1-\bar{k})} \boldsymbol{r}^{*} \boldsymbol{r}^{\top}\right]+\boldsymbol{g}_{I I}^{\top}\left(\frac{1}{n} \boldsymbol{J}_{(N-n) \times n} \otimes \boldsymbol{I}_{N}\right)\right\} \overleftrightarrow{\boldsymbol{Y}}_{I}$,

em que $\boldsymbol{r}^{*}$ é um vetor $N$-dimensional com a $s$-ésima componente igual a $r_{s}^{*}=\frac{N}{N-1} y_{s}(1-$ $\left.k_{s}\right)$.

Usando a expressão (2.60) podemos concluir que:

i) Os estimadors para a média e para o total populacional são respectivamente,

$$
\hat{\mu}=\frac{1}{n} \mathbf{1}_{N n}^{\top} \stackrel{\leftrightarrow}{\boldsymbol{Y}}_{I}=\frac{1}{n} \sum_{i=1}^{n} \sum_{s=1}^{N} U_{i s}\left(y_{s}+W_{s}+\tilde{W}_{i}\right)=\frac{1}{n} \sum_{i=1}^{n} \tilde{Y}_{i} \quad \text { e } \hat{T}=N \hat{\mu}
$$


ii) O estimador do parâmetro individual $y_{j}$ é

$$
\hat{y}_{j}=\frac{N}{n}\left(\mathbf{1}_{n}^{\top} \otimes \boldsymbol{e}_{j}^{\top}\right) \stackrel{\leftrightarrow}{\boldsymbol{Y}}_{I}=\frac{N}{n} \sum_{i=1}^{n} U_{i j}\left(y_{j}+W_{j}+\tilde{W}_{i}\right)
$$

ou seja, $\hat{y}_{j}$ estima o parâmetro com $\frac{N}{n}\left(y_{j}+W_{j}+\tilde{W}_{i}\right)$ quando a unidade $j$ é observada na amostra e com zero em caso contrário; este estimador é do tipo Horvitz-Thompson.

iii) O preditor do valor latente da unidade selecionada na $i$-ésima posição da amostra é

$$
\hat{Y}_{i}=\left\{\boldsymbol{e}_{i I}^{\top} \otimes \mathbf{1}_{N}^{\top}-\boldsymbol{e}_{i I}^{\top} \boldsymbol{P}_{n}^{\top} \otimes\left(x_{1}, x_{2}, \ldots, x_{N}\right)\right\} \overleftrightarrow{\boldsymbol{Y}}_{I}, \quad \text { se } \quad i \leq n
$$

em que

$$
x_{s}=\left(1-k_{s}\right)\left[1+\frac{y_{s}}{\sigma_{s}^{2}} T\right] \text { e } T=\frac{\sum_{s=1}^{N} y_{s}\left(1-k_{s}\right)}{(N-1)(1-\bar{k})}
$$

$\mathrm{ou}$

$$
\hat{Y}_{i}=\frac{1}{n} \sum_{i=1}^{n} \sum_{s=1}^{N} U_{i s}\left(y_{s}+W_{s}+\tilde{W}_{i}\right)=\frac{1}{n} \sum_{i=1}^{n} \tilde{Y}_{i} \text { se } i>n .
$$

Apesar de o preditor (2.61) levar em conta a variabilidade endógena do individuo, $\sigma_{s}^{2}$, note que para fins práticos sua aplicação é restringida porque também se requer o conhecimento a priori de todos os parâmetros individuais $y_{s}, s=1, \ldots, N$, para calcular o valor da constante $T$. Salientamos que mesmo observando a todos os indivíduos da população, o valor do parâmetro individual não será conhecido dado que as medidas são feitas com os dois tipos de erros de medida.

Os coeficientes do estimador/preditor (2.61) mudam para cada população, mostrando que não existe um estimador linear, não enviesado e com variância uniformemente mínima para qualquer população com valores latentes $\boldsymbol{y}$, obtendo assim uma generalização do resultado de Godambe (1955), que mostrou que para populações finitas não existe um estimador não enviesado com variância uniformemente mínima para o total populacional na classe geral de estimadores lineares, i.e., em que os coeficientes atribuídos às respostas observadas dependem tanto da unidade quanto da ordem de seleção na amostra selecionada, ou seja, exatamente a informação contida no vetor $\overleftrightarrow{\boldsymbol{Y}}_{I}$. 


\subsubsection{Unicidade do BLUP de $Y_{i}=\mu+B_{i}$ usando as variáveis aleatórias $\tilde{\boldsymbol{Y}}$}

Quando usamos as variáveis aleatórias $\tilde{\boldsymbol{Y}}$ para obter o BLUP de $P_{i}=\mu+B_{i}$, usamos o critério de otimalidade que consiste em minimizar o erro quadrático médio dos preditores lineares não enviesados. Rao \& Bellhouse (1978) estabelecem condições sob as quais um estimador é ótimo na classe dos estimadores lineares não enviesados. ${ }^{2}$ Vamos verificar se $\hat{P}_{i}$ satisfaz essas condições que são expressas no seguinte teorema.

Teorema 2.2.3. [Teorema 1.1 de Rao $\&$ Bellhouse (1978)] Um estimador $\hat{P}_{i}$ na classe $C$ de estimadores lineares $\xi_{1} \xi_{2} \xi_{3}$-não enviesados de $P_{i}$ baseado em $\tilde{\boldsymbol{Y}}_{I}$ é ótimo para $P_{i}$ se e somente se para todo estimador $\hat{e}_{d}$ de zero, é tal que

$$
\mathbb{E}_{\xi_{1} \xi_{2} \xi_{3}}\left[\left(\hat{P}_{i}-P_{i}\right) \hat{e}_{d}\right]=0 .
$$

Definimos $C_{0}$ como a classe de preditores lineares $\hat{e_{d}}=\boldsymbol{b}^{\top}\left[\left(\boldsymbol{I}_{N} \otimes \boldsymbol{P}_{N}\right) \overleftrightarrow{\boldsymbol{Y}}_{I}\right] \xi_{1} \xi_{2} \xi_{3^{-}}$ não enviesados de zero, i.e., tais que $\mathbb{E}_{\xi_{1} \xi_{2} \xi_{3}}\left(\hat{e}_{d}\right)=0$. Identificamos as restrições lineares necessárias para que $\hat{P}_{i}$ seja não enviesado de $P_{i}$, e para que $\hat{e}_{d}$ seja um predictor linear não enviesado de zero. Em seguida, usamos essas restrições para determinar quando $\mathbb{E}_{\xi_{1} \xi_{2} \xi_{3}}\left[\left(\hat{P}_{i}-P_{i}\right) \hat{e}_{d}\right]$ é igual a zero.

Como o preditor de $P_{i}$ é linear na amostra, temos $\hat{P}_{i}=\left(\boldsymbol{e}_{i I}^{\top}+\boldsymbol{a}^{\top}\right) \tilde{\boldsymbol{Y}}_{I}$. Além disso, como $\hat{P}_{i}$ é não enviesado, então $\boldsymbol{a}$ deve satisfazer $\mathbb{E}_{\xi_{1} \xi_{2} \xi_{3}}\left[\left(\boldsymbol{e}_{i I}^{\top}+\boldsymbol{a}^{\top}\right) \tilde{\boldsymbol{Y}}_{I}-\boldsymbol{e}_{i I}^{\top} \boldsymbol{Y}_{I}-\boldsymbol{e}_{i I I}^{\top} \boldsymbol{Y}_{I I}\right]=0$, implicando que

$$
\boldsymbol{a}^{\top} \mathbf{1}_{n}-\boldsymbol{e}_{i I I}^{\top} \mathbf{1}_{N-n}=\mathbf{0} .
$$

Para que $\hat{e}_{d}$ seja não enviesado para o valor zero, é precisso que $\frac{1}{N} \boldsymbol{b}^{\top}\left(\mathbf{1}_{n} \otimes \boldsymbol{P}_{N}\right) \boldsymbol{y}=0$ para todo $\boldsymbol{y}$; isto implica que

$$
\left(\mathbf{1}_{n}^{\top} \otimes \boldsymbol{P}_{N}\right) \boldsymbol{b}=\mathbf{0}
$$

Logo,

$$
\begin{aligned}
\mathbb{E}_{\xi_{1} \xi_{2} \xi_{3}}\left[\left(\hat{P}_{i}-P_{i}\right) \hat{e}_{d}\right] & =\mathbb{E}_{\xi_{1} \xi_{2} \xi_{3}}\left[\left(\left(\boldsymbol{e}_{i I}^{\top}+\boldsymbol{a}^{\top}\right) \tilde{\boldsymbol{Y}}_{I}-\boldsymbol{e}_{i I}^{\top} \boldsymbol{Y}_{I}-\boldsymbol{e}_{i I I}^{\top} \boldsymbol{Y}_{I I}\right) \overleftrightarrow{\boldsymbol{Y}}_{I}^{\top}\left(\boldsymbol{I}_{n} \otimes \boldsymbol{P}_{N}\right) \boldsymbol{b}\right] \\
= & {\left[\left(\boldsymbol{e}_{i I}^{\top}+\boldsymbol{a}^{\top}\right)\left|-\boldsymbol{e}_{i I}^{\top}\right|-\boldsymbol{e}_{i I I}^{\top}\right] \mathbb{E}_{\xi_{1} \xi_{2} \xi_{3}}\left[\left(\begin{array}{c}
\tilde{\boldsymbol{Y}}_{I} \\
\boldsymbol{Y}_{I} \\
\boldsymbol{Y}_{I I}
\end{array}\right) \stackrel{\leftrightarrow}{\boldsymbol{Y}}_{I}^{\top}\right]\left(\boldsymbol{I}_{n} \otimes \boldsymbol{P}_{N}\right) \boldsymbol{b} . }
\end{aligned}
$$

\footnotetext{
2 "Ótimo" no sentido de que tem o menor erro quadrático médio.
} 
Dado que

$$
\left(\begin{array}{c}
\tilde{\boldsymbol{Y}}_{I} \\
\boldsymbol{Y}_{I} \\
\boldsymbol{Y}_{I I}
\end{array}\right)=\left(\begin{array}{ccc}
\boldsymbol{I}_{n} \otimes \mathbf{1}_{N}^{\top} & \mathbf{0}_{n \times n N} & \mathbf{0}_{n \times N(N-n)} \\
\mathbf{0}_{n \times n N} & \boldsymbol{I}_{n} \otimes \mathbf{1}_{N}^{\top} & \mathbf{0}_{n \times N(N-n)} \\
\mathbf{0}_{(N-n) \times n N} & \mathbf{0}_{(N-n) \times n N} & \boldsymbol{I}_{N-n} \otimes \mathbf{1}_{N}^{\top}
\end{array}\right)\left(\begin{array}{c}
\overleftrightarrow{\boldsymbol{Y}}_{I} \\
\ddot{\boldsymbol{Y}}_{I} \\
\ddot{\boldsymbol{Y}}_{I I}
\end{array}\right)=\boldsymbol{M}\left(\begin{array}{c}
\overleftrightarrow{\boldsymbol{Y}}_{I} \\
\ddot{\boldsymbol{Y}}_{I} \\
\ddot{\boldsymbol{Y}}_{I I}
\end{array}\right)
$$

temos que

$$
\mathbb{E}_{\xi_{1} \xi_{2} \xi_{3}}\left[\left(\begin{array}{c}
\tilde{\boldsymbol{Y}}_{I} \\
\boldsymbol{Y}_{I} \\
\boldsymbol{Y}_{I I}
\end{array}\right) \stackrel{\leftrightarrow}{\boldsymbol{Y}}_{I}^{\top}\right]=\boldsymbol{M} \mathbb{E}_{\xi_{1} \xi_{2} \xi_{3}}\left[\left(\begin{array}{c}
\overleftrightarrow{\boldsymbol{Y}}_{I} \\
\ddot{\boldsymbol{Y}}_{I} \\
\ddot{\boldsymbol{Y}}_{I I}
\end{array}\right) \stackrel{\leftrightarrow}{\boldsymbol{Y}}_{I}^{\top}\right]
$$

Lembrando que $\mathbb{V}[\boldsymbol{x}]=\mathbb{E}\left[\boldsymbol{x} \boldsymbol{x}^{\top}\right]-\mathbb{E}[\boldsymbol{x}] \mathbb{E}\left[\boldsymbol{x}^{\top}\right]$, então

$$
\mathbb{E}_{\xi_{1} \xi_{2} \xi_{3}}\left[\left(\begin{array}{c}
\overleftrightarrow{\boldsymbol{Y}}_{I} \\
\ddot{\boldsymbol{Y}}_{I} \\
\ddot{\boldsymbol{Y}}_{I I}
\end{array}\right) \stackrel{\boldsymbol{Y}}{I}^{\top}\right]
$$

são as primeiras $n N$ colunas da expressão

$$
\mathbb{V}_{\xi_{1} \xi_{2} \xi_{3}}\left(\begin{array}{c}
\overleftrightarrow{\boldsymbol{Y}}_{I} \\
\ddot{\boldsymbol{Y}}_{I} \\
\ddot{\boldsymbol{Y}}_{I I}
\end{array}\right)+\mathbb{E}_{\xi_{1} \xi_{2} \xi_{3}}\left(\begin{array}{c}
\overleftrightarrow{\boldsymbol{Y}}_{I} \\
\ddot{\boldsymbol{Y}}_{I} \\
\ddot{\boldsymbol{Y}}_{I I}
\end{array}\right) \mathbb{E}_{\xi_{1} \xi_{2} \xi_{3}}\left[\left(\begin{array}{c}
\overleftrightarrow{\boldsymbol{Y}}_{I} \\
\ddot{\boldsymbol{Y}}_{I} \\
\ddot{\boldsymbol{Y}}_{I I}
\end{array}\right)\right]
$$

Da expressão (A.13) sabemos que

$$
\mathbb{V}_{\xi_{1} \xi_{2} \xi_{3}}\left[\left(\begin{array}{c}
\overleftrightarrow{\boldsymbol{Y}}_{I} \\
\ddot{\boldsymbol{Y}}_{I} \\
\ddot{\boldsymbol{Y}}_{I I}
\end{array}\right)\right]=\left(\begin{array}{ccc}
\stackrel{\boldsymbol{V}}{I}_{I} & \boldsymbol{V}_{I} & \boldsymbol{V}_{I, I I} \\
\boldsymbol{V}_{I} & \boldsymbol{V}_{I} & \boldsymbol{V}_{I, I I} \\
\boldsymbol{V}_{I I, I} & \boldsymbol{V}_{I I, I} & \boldsymbol{V}_{I I}
\end{array}\right),
$$

com

$$
\begin{aligned}
\boldsymbol{V}_{I} & =\left(\boldsymbol{I}_{n}-\frac{1}{N} \boldsymbol{J}_{n}\right) \otimes \Delta \\
\boldsymbol{V}_{I, I I} & =-\frac{1}{N} \boldsymbol{J}_{n \times(N-n)} \otimes \Delta \\
\boldsymbol{V}_{I I} & =\left(\boldsymbol{I}_{N-n}-\frac{1}{N} \boldsymbol{J}_{N-n}\right) \otimes \Delta \\
\overleftrightarrow{\boldsymbol{V}}_{I} & =\boldsymbol{V}_{I}+\frac{1}{N} \boldsymbol{I}_{n} \otimes\left(\bigoplus_{s=1}^{N} \sigma_{s}^{2}\right)+\frac{1}{N}\left(\bigoplus_{i=1}^{n} \tilde{\sigma}_{i}^{2}\right) \otimes \boldsymbol{I}_{N} .
\end{aligned}
$$


Adicionalmente, como

$$
\mathbb{E}_{\xi_{1} \xi_{2} \xi_{3}}\left(\begin{array}{c}
\overleftrightarrow{\boldsymbol{Y}}_{I} \\
\ddot{\boldsymbol{Y}}_{I} \\
\ddot{\boldsymbol{Y}}_{I I}
\end{array}\right)=\left(\begin{array}{c}
\frac{1}{N}\left(\mathbf{1}_{n} \otimes \boldsymbol{y}\right) \\
\frac{1}{N}\left(\mathbf{1}_{n} \otimes \boldsymbol{y}\right) \\
\frac{1}{N}\left(\mathbf{1}_{N-n} \otimes \boldsymbol{y}\right)
\end{array}\right)
$$

então

$$
\mathbb{E}_{\xi_{1} \xi_{2} \xi_{3}}\left[\left(\begin{array}{c}
\stackrel{\leftrightarrow}{\boldsymbol{Y}}_{I} \\
\ddot{\boldsymbol{Y}}_{I} \\
\ddot{\boldsymbol{Y}}_{I I}
\end{array}\right) \overleftrightarrow{\boldsymbol{Y}}_{I}^{\top}\right]=\left(\begin{array}{c}
\stackrel{\boldsymbol{V}}{I}^{\top} \\
\boldsymbol{V}_{I} \\
\boldsymbol{V}_{I I, I}
\end{array}\right)+\frac{1}{N^{2}}\left(\begin{array}{c}
\mathbf{1}_{n} \mathbf{1}_{n}^{\top} \otimes \boldsymbol{y} \boldsymbol{y}^{\top} \\
\mathbf{1}_{n} \mathbf{1}_{n}^{\top} \otimes \boldsymbol{y} \boldsymbol{y}^{\top} \\
\mathbf{1}_{N-n} \mathbf{1}_{n}^{\top} \otimes \boldsymbol{y} \boldsymbol{y}^{\top}
\end{array}\right)=\left(\begin{array}{c}
A \\
B \\
C
\end{array}\right)
$$

em que

$$
\begin{aligned}
A & =\left(\boldsymbol{I}_{n}-\frac{1}{N} \boldsymbol{J}_{n}\right) \otimes \Delta+\frac{1}{N} \boldsymbol{I}_{n} \otimes\left(\bigoplus_{s=1}^{N} \sigma_{s}^{2}\right)+\frac{1}{N}\left(\bigoplus_{i=1}^{n} \tilde{\sigma}_{i}^{2}\right) \otimes \boldsymbol{I}_{N}+\frac{1}{N^{2}}\left(\boldsymbol{J}_{n} \otimes \boldsymbol{y} \boldsymbol{y}^{\top}\right), \\
B & =\left(\boldsymbol{I}_{n}-\frac{1}{N} \boldsymbol{J}_{n}\right) \otimes \Delta+\frac{1}{N^{2}}\left(\boldsymbol{J}_{n} \otimes \boldsymbol{y} \boldsymbol{y}^{\top}\right), \\
C & =-\frac{1}{N} \boldsymbol{J}_{(N-n) \times n} \otimes \Delta+\frac{1}{N^{2}}\left(\boldsymbol{J}_{n} \otimes \boldsymbol{y} \boldsymbol{y}^{\top}\right) .
\end{aligned}
$$

Assim temos

$$
\mathbb{E}_{\xi_{1} \xi_{2} \xi_{3}}\left[\left(\begin{array}{c}
\tilde{\boldsymbol{Y}}_{I} \\
\boldsymbol{Y}_{I} \\
\boldsymbol{Y}_{I I}
\end{array}\right) \stackrel{\leftrightarrow}{\boldsymbol{Y}}_{I}^{\top}\right]=\left(\begin{array}{c}
D \\
E \\
F
\end{array}\right)
$$

em que

$$
\begin{aligned}
D & =\left(\boldsymbol{I}_{n}-\frac{1}{N} \boldsymbol{J}_{n}\right) \otimes \mathbf{1}_{N}^{\top} \Delta+\frac{1}{N} \boldsymbol{I}_{n} \otimes \mathbf{1}_{N}^{\top}\left(\bigoplus_{s=1}^{N} \sigma_{s}^{2}\right)+\frac{1}{N}\left(\bigoplus_{i=1}^{n} \tilde{\sigma}_{i}^{2}\right) \otimes \mathbf{1}_{N}^{\top} \\
& +\frac{1}{N^{2}}\left(\boldsymbol{J}_{n} \otimes \mathbf{1}_{N}^{\top} \boldsymbol{y} \boldsymbol{y}^{\top}\right), \\
E & =\left(\boldsymbol{I}_{n}-\frac{1}{N} \boldsymbol{J}_{n}\right) \otimes \mathbf{1}_{N}^{\top} \Delta+\frac{1}{N^{2}}\left(\boldsymbol{J}_{n} \otimes \mathbf{1}_{N}^{\top} \boldsymbol{y} \boldsymbol{y}^{\top}\right), \\
F & =-\frac{1}{N} \boldsymbol{J}_{(N-n) \times n} \otimes \mathbf{1}_{N}^{\top} \Delta+\frac{1}{N^{2}}\left(\boldsymbol{J}_{n} \otimes \mathbf{1}_{N}^{\top} \boldsymbol{y} \boldsymbol{y}^{\top}\right) .
\end{aligned}
$$

Agora, determinamos o valor de $\mathbb{E}_{\xi_{1} \xi_{2} \xi_{3}}\left[\left(\hat{P}_{i}-P_{i}\right) \hat{e}_{d}\right]$ sujeito às restrições $(2.63)$ e (2.64). De (2.65) temos

$$
\mathbb{E}_{\xi_{1} \xi_{2} \xi_{3}}\left[\left(\hat{P}_{i}-P_{i}\right) \hat{e}_{d}\right]=\left(\left(\boldsymbol{e}_{i I}^{\top}+\boldsymbol{a}^{\top}\right)\left|-\boldsymbol{e}_{i I}^{\top}\right|-\boldsymbol{e}_{i I I}^{\top}\right)\left(\begin{array}{c}
D \\
E \\
F
\end{array}\right)\left(\boldsymbol{I}_{n} \otimes \boldsymbol{P}_{N}\right) \boldsymbol{b} .
$$


Aplicando (2.63) e (2.64) segue que

$$
\begin{aligned}
\left(\boldsymbol{J}_{n} \otimes \mathbf{1}_{N}^{\top} \boldsymbol{y} \boldsymbol{y}^{\top}\right)\left(\boldsymbol{I}_{n} \otimes \boldsymbol{P}_{N}\right) \boldsymbol{b} & =\left(\mathbf{1}_{n} \otimes \mathbf{1}_{N}^{\top} \boldsymbol{y} \boldsymbol{y}^{\top}\right)\left(\mathbf{1}_{n}^{\top} \otimes \boldsymbol{P}_{N}\right) \boldsymbol{b}=0, \\
\left(\boldsymbol{J}_{(N-n) \times n} \otimes \mathbf{1}_{N}^{\top} \boldsymbol{y} \boldsymbol{y}^{\top}\right)\left(\boldsymbol{I}_{n} \otimes \boldsymbol{P}_{N}\right) \boldsymbol{b} & =\left(\mathbf{1}_{N-n} \otimes \mathbf{1}_{N}^{\top} \boldsymbol{y} \boldsymbol{y}^{\top}\right)\left(\mathbf{1}_{n}^{\top} \otimes \boldsymbol{P}_{N}\right) \boldsymbol{b}=0, \\
\left(\boldsymbol{J}_{n} \otimes \mathbf{1}_{N}^{\top} \Delta\right)\left(\boldsymbol{I}_{n} \otimes \boldsymbol{P}_{N}\right) \boldsymbol{b} & =\left(\mathbf{1}_{n} \otimes \mathbf{1}_{N}^{\top} \Delta\right)\left(\mathbf{1}_{n}^{\top} \otimes \boldsymbol{P}_{N}\right) \boldsymbol{b}=0, \\
\left(\boldsymbol{J}_{(N-n) \times n} \otimes \mathbf{1}_{N}^{\top} \Delta\right)\left(\boldsymbol{I}_{n} \otimes \boldsymbol{P}_{N}\right) \boldsymbol{b} & =\left(\mathbf{1}_{N-n} \otimes \mathbf{1}_{N}^{\top} \Delta\right)\left(\mathbf{1}_{n}^{\top} \otimes \boldsymbol{P}_{N}\right) \boldsymbol{b}=0, \\
\left(\left(\oplus_{i=1}^{n} \tilde{\sigma}_{i}^{2}\right) \otimes \mathbf{1}_{N}^{\top}\right)\left(\boldsymbol{I}_{n} \otimes \boldsymbol{P}_{N}\right) \boldsymbol{b} & =\left(\left(\oplus_{i=1}^{n} \tilde{\sigma}_{i}^{2}\right) \otimes \mathbf{1}_{N}^{\top} \boldsymbol{P}_{N}\right) \boldsymbol{b}=0 .
\end{aligned}
$$

Uma vez que $\mathbf{1}_{N}^{\top} \boldsymbol{P}_{N}=0$, obtemos,

$$
\begin{aligned}
\mathbb{E}_{\xi_{1} \xi_{2} \xi_{3}}\left[\left(\hat{P}_{i}-P_{i}\right) \hat{e}_{d}\right] & =\left(\boldsymbol{e}_{i I}^{\top}\right)\left\{\frac{1}{N} \boldsymbol{I}_{n} \otimes \mathbf{1}_{N}^{\top}\left(\bigoplus_{s=1}^{N} \sigma_{s}^{2}\right) \boldsymbol{P}_{N}\right\} \boldsymbol{b} \\
& +\left(\boldsymbol{a}^{\top}\right)\left\{\boldsymbol{I}_{n} \otimes \mathbf{1}_{N}^{\top} \Delta \boldsymbol{P}_{N}+\frac{1}{N} \boldsymbol{I}_{n} \otimes \mathbf{1}_{N}^{\top}\left(\bigoplus_{s=1}^{N} \sigma_{s}^{2}\right) \boldsymbol{P}_{N}\right\} \boldsymbol{b} .
\end{aligned}
$$

Quando $i \leq n$, temos $\boldsymbol{e}_{i I I}=\mathbf{0}$, e então $\boldsymbol{a}^{\top}=\mathbf{0}$; consequentemente

$$
\mathbb{E}_{\xi_{1} \xi_{2} \xi_{3}}\left[\left(\hat{P}_{i}-P_{i}\right) \hat{e}_{d}\right]=\left(\boldsymbol{e}_{i I}^{\top}\right)\left\{\frac{1}{N} \boldsymbol{I}_{n} \otimes \mathbf{1}_{N}^{\top}\left(\bigoplus_{s=1}^{N} \sigma_{s}^{2}\right) \boldsymbol{P}_{N}\right\} \boldsymbol{b} .
$$

$\mathbb{E}_{\xi_{1} \xi_{2} \xi_{3}}\left[\left(\hat{P}_{i}-P_{i}\right) \hat{e}_{d}\right]=0$ se e somente se $\left(\bigoplus_{s=1}^{N} \sigma_{s}^{2}\right)=k \boldsymbol{I}_{N}$, i.e., $\hat{P}_{i}$ será ótimo quando $\sigma_{s}^{2}=k, s=1, \ldots, N$. Sabemos que $\mathbb{V}_{\xi_{1} \xi_{2} \xi_{3}}(\tilde{\boldsymbol{Y}})=\gamma^{2} \boldsymbol{P}_{N}+\bar{\sigma}^{2} \boldsymbol{I}_{N}+\bigoplus_{i=1}^{N} \tilde{\sigma}_{i}^{2}$; logo quando usamos as variáveis $\tilde{\boldsymbol{Y}}$ temos $k=\bar{\sigma}^{2}=N^{-1} \sum_{s=1}^{N} \sigma_{s}^{2}$. Portanto, o preditor é ótimo para $i \leq n$.

Quando $i>n$, temos $\boldsymbol{e}_{i I}=\mathbf{0}, \boldsymbol{a}^{\top} \mathbf{1}_{n}=1, \boldsymbol{a}^{\top} \neq \mathbf{0}$, e

$$
\mathbb{E}_{\xi_{1} \xi_{2} \xi_{3}}\left[\left(\hat{P}_{i}-P_{i}\right) \hat{e}_{d}\right]=\left(\boldsymbol{a}^{\top}\right)\left\{\boldsymbol{I}_{n} \otimes \mathbf{1}_{N}^{\top} \Delta \boldsymbol{P}_{N}+\frac{1}{N} \boldsymbol{I}_{n} \otimes \mathbf{1}_{N}^{\top}\left(\bigoplus_{s=1}^{N} \sigma_{s}^{2}\right) \boldsymbol{P}_{N}\right\} \boldsymbol{b} .
$$

Então, $\mathbb{E}_{\xi_{1} \xi_{2} \xi_{3}}\left[\left(\hat{P}_{i}-P_{i}\right) \hat{e}_{d}\right]=0$ se e somente se $\Delta=m \boldsymbol{P}_{N}$ e $\left(\bigoplus_{s=1}^{N} \sigma_{s}^{2}\right)=k \boldsymbol{I}_{N}$. Sabemos que $\mathbb{V}_{\xi_{1} \xi_{2} \xi_{3}}(\tilde{\boldsymbol{Y}})=\gamma^{2} \boldsymbol{P}_{N}+\bar{\sigma}^{2} \boldsymbol{I}_{N}+\bigoplus_{i=1}^{N} \tilde{\sigma}_{i}^{2}$. De forma equivalente, quando usamos as variáveis $\tilde{\boldsymbol{Y}}$, temos $m=\gamma^{2}$ e $k=\bar{\sigma}^{2}$, portanto, $\hat{P}_{i}$ é ótimo para $i>n$.

Então, $\mathbb{E}_{\xi_{1} \xi_{2} \xi_{3}}\left[\left(\hat{P}_{i}-P_{i}\right) \hat{e}_{d}\right]=0$ para todo $i$, portanto $\hat{P}_{i}$ é ótimo para todo $i$, i.e., $\hat{P}_{i}$ é o preditor linear não enviesado que tem o menor erro quadrático médio nessa classe de preditores. 


\subsection{Discussão}

O BLUP para $Y_{i}=\mu+B_{i}$ obtido a partir das variáveis aleatórias $\tilde{\boldsymbol{Y}}$ é ótimo, mas não leva em conta a informação da variabilidade endógena individual $\sigma_{s}^{2}$. Por outro lado, o BLUP para $Y_{i}=\mu+B_{i}$ obtido a partir das variáveis aleatórias expandidas $\overleftrightarrow{\boldsymbol{Y}}$ usa a informação da variabilidade endógena individual $\sigma_{s}^{2}$, mas depende do conhecimento de todos os valores latentes $y_{1}, \ldots, y_{N}$ e por isso não tem utilidade prática.

Quando usamos o modelo misto expandido com erros de medida endógeno e exógeno, o estimador do valor latente da unidade com etiqueta $s$ na população é $\frac{N}{n}\left(y_{s}+W_{s}+\tilde{W}_{i}\right)$ quando a unidade $s$ é observada na posição $i$ da amostra e zero em caso contrário. Este estimador não parece ser atrativo, porque apesar de que os erros de medida endógenos e exógenos sejam nulos $\left(W_{s}=\tilde{W}_{i}=0\right)$ o estimador para as unidades observadas $\left(\frac{N}{n} y_{s}\right)$ é diferente do verdadeiro valor latente $y_{s}$. Para unidades não observadas o estimador assume um valor nulo, porque o conhecimento de um parâmetro individual não contribui para o conhecimento dos demais parâmetros.

Por outro lado, o modelo misto para populações finitas com erros de medida endógeno e exógeno é gerado a partir das variáveis aleatórias indicadoras $U_{i s}$; com elas é possível determinar se a unidade com etiqueta $s$ na população está ou não na amostra, isto é, se $s \in \mathfrak{S}=\left\{s_{1}, \ldots, s_{n}\right\}$, então

$$
\sum_{s=1}^{N} s U_{i s}=s_{i}, \text { para algum } i,
$$

por conseguinte, depois de obter a amostra, o BLUP do valor latente da unidade na $i$-ésima posição é

$$
\hat{Y}_{s_{i}}=\overline{\bar{Y}}+\frac{\gamma^{2}}{\gamma^{2}+\bar{\sigma}^{2}+\tilde{\sigma}_{i}^{2}}\left(\tilde{Y}_{s_{i}}-\overline{\bar{Y}}\right), s_{i} \in\left\{s_{1}, \ldots, s_{n}\right\},
$$

ou $\hat{Y}_{s_{i}}=\overline{\bar{Y}}$ para $s_{i} \notin \mathfrak{S}=\left\{s_{1}, \ldots, s_{n}\right\}$. Isto é, o preditor (2.69) contém a etiqueta da unidade da população seleccionada na $i$-ésima posição.

Neste capítulo, mostramos que os resultados de Stanek III et al. (2004) e Stanek III \& Singer (2004) podem ser estendidos para acomodar erros de medida endógenos e exógenos simultaneamente no modelo misto para populações finitas. O modelo misto para populações finitas pode ser empregado tanto para encontrar estimadores de uma média populacional ou de um total populacional, quanto para predizer o valor latente da unidade 
na $i$-ésima posição numa permutação ou da unidade com etiqueta $s$ na população. Os estimadores/preditores são obtidos via métodos padrão para encontrar preditores lineares não enviesados de variância mínima. 


\section{Capítulo 3}

\section{Comparação entre preditores obtidos sob os modelos mistos usual e para populações finitas}

O objetivo deste capítulo é comparar preditores dos valores latentes das unidades amostrais obtidos a partir do modelo misto usual e do modelo misto para populações finitas com relação ao erro quadrático médio numa situação em que há erros de medida endógenos heterocedásticos.

\section{$3.1 \quad$ Exemplo}

Inicialmente, consideramos uma situação análoga àquela apresentada em Singer, Stanek III, Lencina, González, Li \& San Martino (2009). Por simplicidade, consideramos uma população de $N=3$ indivíduos da qual selecionamos uma amostra aleatória simples de tamanho $n=2$, fazendo uma única medição do nível de colesterol em cada unidade amostral. Desejamos predizer o valor latente de colesterol de cada indivíduo na amostra usando tanto o preditor obtido a partir do modelo misto usual $\left(\hat{Q}_{i}^{(1)}\right)$ quanto o preditor obtido a partir do MMPF $\left(\hat{Q}_{i}^{(2)}\right)$. A Tabela 3.1 contém os valores latentes $y_{s}$, as variâncias do erro de medida endógeno, $\sigma_{s}^{2}$, que assumimos conhecidas e as constantes de encolhimento $k$ e $k_{s}, s=1,2,3$, necessárias para o cálculo dos dois preditores. Assumimos que o erro de medida pode ter somente dois possíveis valores igualmente prováveis e dados por $\pm \sigma_{s}$. 
Tabela 3.1: Nível de colesterol, variâncias endógenas, constantes de encolhimento e de ponderação na população $(N=3)$.

\begin{tabular}{lclcc}
\hline Nome & $\begin{array}{c}\text { Nível de colesterol* } \\
\text { (valor latente) }\end{array}$ & Variância & $\begin{array}{c}\text { Constante de } \\
\text { encolhimento }\end{array}$ & $\begin{array}{c}\text { Constante de } \\
\text { Ponderação }\end{array}$ \\
\hline Alba & $y_{1}=10$ & $\sigma_{1}^{2}=1$ & $k_{1}=0,950$ & $w_{1}=0,491$ \\
Juliana & $y_{2}=3$ & $\sigma_{2}^{2}=100$ & $k_{2}=0,160$ & $w_{2}=0,082$ \\
Laura & $y_{3}=2$ & $\sigma_{3}^{2}=4$ & $k_{3}=0,826$ & $w_{3}=0,427$ \\
\hline & $\mu=5$ & & & \\
& $\gamma^{2}=19$ & $\bar{\sigma}^{2}=35$ & $k=0,352$ & \\
\hline
\end{tabular}

*Nível de colesterol depois de subtraídas 200 unidades.

Para todas as possíveis 24 amostras, ${ }^{1}$ a resposta observada $y_{s} \pm \sigma_{s}$, os valores preditos $\hat{Q}_{i}^{(1)}$ e o quadrado da diferença entre o valor latente e o valor predito, $\left(\hat{Q}_{i}^{(1)}-y_{i}\right)^{2}, i=1,2$, são apresentados na Tabela 3.2. A média dos valores correspondentes às 24 amostras está apresentada na última linha.

Lembramos aqui que o preditor obtido a partir do modelo misto usual,

$$
\hat{Q}_{i}^{(1)}=\hat{\mu}+\frac{\gamma^{2}}{\left(\gamma^{2}+\sigma_{i}^{2}\right)}\left(Y_{i}-\hat{\mu}\right)
$$

em que $\hat{\mu}=\sum_{i=1}^{n} w_{i} Y_{i} / \sum_{i=1}^{n} w_{i}, w_{i}=\left(\gamma^{2}+\sigma_{i}^{2}\right)^{-1}$ e $k_{i}=\gamma^{2} /\left(\gamma^{2}+\sigma_{i}^{2}\right)$, foi utilizado conforme a interpretação usual (preditor do colesterol do $i$-ésimo indivíduo selecionado). Essa forma de interpretar esse preditor não é correta, pois $\sigma_{i}^{2}$ é constante em relação à posição na amostra $(i)$ mas varia de acordo com o indivíduo selecionado nessa posição. Como consequência, a média dos valores preditos por (3.1) para as 24 possíveis amostras é 5,8 , o que indica que esse preditor é enviesado pois a média populacional é 5,0 . Isto mostra que o preditor $\hat{Q}_{i}^{(1)}$ calculado segundo a interpretação usual não é o BLUP.

Na Tabela 3.3, apresentamos novamente a resposta observada $y_{s} \pm \sigma_{s}$, os valores preditos em cada posição usando o preditor

$$
\hat{Q}_{i}^{(2)}=\bar{Y}+\frac{\gamma^{2}}{\gamma^{2}+\bar{\sigma}^{2}}\left(Y_{i}-\bar{Y}\right)
$$

\footnotetext{
${ }^{1}$ As 6 combinações de dois indivíduos com as 4 possíveis combinações de erros de medida, $\pm \sigma_{s_{1}} \pm \sigma_{s_{2}}$
} 
Tabela 3.2: Preditor obtido a partir dos modelos mistos usuais com variâncias heterocedásticas.

\begin{tabular}{|c|c|c|c|c|c|c|c|c|}
\hline \multirow{2}{*}{$\frac{\text { Amostra }}{1}$} & \multirow{2}{*}{$\frac{y_{s_{1}}}{\text { lba }(10)}$} & \multirow{2}{*}{$\frac{y_{s_{2}}}{\text { Juliana }(3)}$} & \multicolumn{2}{|c|}{$Y_{i}=y_{s_{i}} \pm \sigma_{s_{i}}$} & \multicolumn{2}{|c|}{$\hat{Q}_{i}^{(1)}$} & \multicolumn{2}{|c|}{$\left(\hat{Q}_{i}^{(1)}-y_{s_{i}}\right)^{2}$} \\
\hline & & & 11 & 13 & 11,0 & 11,6 & 1,03 & 73,29 \\
\hline 2 & Alba (10) & Juliana (3) & 11 & -7 & 10,9 & 5,9 & 0,76 & 8,70 \\
\hline 3 & Alba (10) & Juliana (3) & 9 & 13 & 9,0 & 10,1 & 0,94 & 50,73 \\
\hline 4 & Alba (10) & Juliana (3) & 9 & -7 & 8,9 & 4,5 & 1,24 & 2,28 \\
\hline 5 & Alba (10) & Laura (2) & 11 & 4 & 10,8 & 4,7 & 0,70 & 7,03 \\
\hline 6 & Alba (10) & Laura (2) & 11 & 0 & 10,7 & 1,0 & 0,55 & 0,95 \\
\hline 7 & Alba (10) & Laura (2) & 9 & 4 & 8,9 & 4,5 & 1,25 & 6,08 \\
\hline 8 & Alba (10) & Laura (2) & 9 & 0 & 8,8 & 0,8 & 1,46 & 1,35 \\
\hline 9 & Juliana (3) & Alba (10) & 13 & 11 & 11,6 & 11,0 & 73,29 & 1,03 \\
\hline 10 & Juliana (3) & Alba (10) & 13 & 9 & 10,1 & 9,0 & 50,73 & 0,94 \\
\hline 11 & Juliana (3) & Alba (10) & -7 & 11 & 5,9 & 10,9 & 8,70 & 0,76 \\
\hline 12 & Juliana (3) & Alba (10) & -7 & 9 & 4,5 & 8,9 & 2,28 & 1,24 \\
\hline 13 & Juliana (3) & Laura (2) & 13 & 4 & 6,7 & 4,3 & 13,41 & 5,08 \\
\hline 14 & Juliana (3) & Laura (2) & 13 & 0 & 3,8 & 0,4 & 0,71 & 2,67 \\
\hline 15 & Juliana (3) & Laura (2) & -7 & 4 & 0,7 & 3,7 & 5,08 & 2,86 \\
\hline 16 & Juliana (3) & Laura (2) & -7 & 0 & $-2,1$ & $-0,2$ & 25,71 & 4,83 \\
\hline 17 & Laura (2) & Alba (10) & 4 & 11 & 4,7 & 10,8 & 7,03 & 0,70 \\
\hline 18 & Laura (2) & Alba (10) & 4 & 9 & 4,5 & 8,9 & 6,08 & 1,25 \\
\hline 19 & Laura (2) & Alba (10) & 0 & 11 & 1,0 & 10,7 & 0,95 & 0,55 \\
\hline 20 & Laura (2) & Alba (10) & 0 & 9 & 0,8 & 8,8 & 1,35 & 1,46 \\
\hline 21 & Laura (2) & Juliana (3) & 4 & 13 & 4,3 & 6,7 & 5,08 & 13,41 \\
\hline 22 & Laura (2) & Juliana (3) & 4 & -7 & 3,7 & 0,7 & 2,86 & 5,08 \\
\hline 23 & Laura (2) & Juliana (3) & 0 & 13 & 0,4 & 3,8 & 2,67 & 0,71 \\
\hline \multirow[t]{2}{*}{24} & Laura (2) & Juliana (3) & 0 & -7 & $-0,2$ & $-2,1$ & 4,83 & 25,71 \\
\hline & \multicolumn{2}{|c|}{ Médias } & 5,0 & 5,0 & 5,8 & 5,8 & 9,11 & 9,11 \\
\hline
\end{tabular}

(*) $y_{s_{i}}$ representa o valor latente do indivíduo na $i$-ésima posição, $i=1,2$. 
Tabela 3.3: Preditor obtido a partir dos modelos mistos para populações finitas com variâncias heterocedásticas.

\begin{tabular}{|c|c|c|c|c|c|c|c|c|}
\hline \multirow{2}{*}{$\frac{\text { Amostra }}{1}$} & \multirow{2}{*}{$\frac{y_{s_{1}}}{\text { lba }(10)}$} & \multirow{2}{*}{$\frac{y_{s_{2}}}{\text { Juliana }(3)}$} & \multicolumn{2}{|c|}{$Y_{i}=y_{s_{i}} \pm \sigma_{s_{i}}$} & \multicolumn{2}{|c|}{$\hat{Q}_{i}^{(2)}$} & \multicolumn{2}{|c|}{$\left(\hat{Q}_{i}^{(2)}-y_{s_{i}}\right)^{2}$} \\
\hline & & & 11 & 13 & 11,6 & 12,4 & 2,72 & 87,46 \\
\hline 2 & Alba (10) & Juliana (3) & 11 & -7 & 5,2 & $-1,2$ & 23,36 & 17,36 \\
\hline 3 & Alba (10) & Juliana (3) & 9 & 13 & 10,3 & 11,7 & 0,09 & 75,75 \\
\hline 4 & Alba (10) & Juliana (3) & 9 & -7 & 3,8 & $-1,8$ & 38,26 & 23,18 \\
\hline 5 & Alba (10) & Laura (2) & 11 & 4 & 8,7 & 6,3 & 1,61 & 18,22 \\
\hline 6 & Alba (10) & Laura (2) & 11 & 0 & 7,4 & 3,6 & 6,58 & 2,45 \\
\hline 7 & Alba (10) & Laura (2) & 9 & 4 & 7,4 & 5,6 & 6,87 & 13,11 \\
\hline 8 & Alba (10) & Laura (2) & 9 & 0 & 6,1 & 2,9 & 15,34 & 0,84 \\
\hline 9 & Juliana (3) & Alba (10) & 13 & 11 & 12,4 & 11,6 & 87,46 & 2,72 \\
\hline 10 & Juliana (3) & Alba (10) & 13 & 9 & 11,7 & 10,3 & 75,75 & 0,09 \\
\hline 11 & Juliana (3) & Alba (10) & -7 & 11 & $-1,2$ & 5,2 & 17,36 & 23,36 \\
\hline 12 & Juliana (3) & Alba (10) & -7 & 9 & 4,5 & 8,9 & 2,28 & 1,24 \\
\hline 13 & Juliana (3) & Laura (2) & 13 & 4 & 10,1 & 6,9 & 50,17 & 24,17 \\
\hline 14 & Juliana (3) & Laura (2) & 13 & 0 & 8,8 & 4,2 & 33,49 & 4,90 \\
\hline 15 & Juliana (3) & Laura (2) & -7 & 4 & $-3,4$ & 0,4 & 41,41 & 2,45 \\
\hline 16 & Juliana (3) & Laura (2) & -7 & 0 & $-4,7$ & $-2,3$ & 59,78 & 18,22 \\
\hline 17 & Laura (2) & Alba (10) & 4 & 11 & 6,3 & 8,7 & 18,22 & 1,61 \\
\hline 18 & Laura (2) & Alba (10) & 4 & 9 & 5,6 & 7,4 & 13,11 & 6,87 \\
\hline 19 & Laura (2) & Alba (10) & 0 & 11 & 3,6 & 7,4 & 2,45 & 6,58 \\
\hline 20 & Laura (2) & Alba (10) & 0 & 9 & 2,9 & 6,1 & 0,84 & 15,34 \\
\hline 21 & Laura (2) & Juliana (3) & 4 & 13 & 6,9 & 10,1 & 24,17 & 50,17 \\
\hline 22 & Laura (2) & Juliana (3) & 4 & -7 & 0,4 & $-3,4$ & 2,45 & 41,41 \\
\hline 23 & Laura (2) & Juliana (3) & 0 & 13 & 4,2 & 8,8 & 4,90 & 33,49 \\
\hline \multirow[t]{2}{*}{24} & Laura (2) & Juliana (3) & 0 & -7 & $-2,3$ & $-4,7$ & 18,22 & 59,78 \\
\hline & \multicolumn{2}{|c|}{ Médias } & 5,0 & 5,0 & 5,0 & 5,0 & 23,66 & 23,66 \\
\hline
\end{tabular}

(*) $y_{s_{i}}$ representa o valor latente do indivíduo na $i$-ésima posição, $i=1,2$. 
em que $\bar{Y}=\sum_{i=1}^{n} Y_{i} / n$ e $k=\gamma^{2} /\left(\gamma^{2}+\sigma^{2}\right)$. A média para as 24 amostras está calculada na última linha. A média dos valores preditos para as 24 possíveis amostras é 5,0 e é igual à média populacional, ou seja, $\hat{Q}_{i}^{(2)}$ é o BLUP. No entanto, o erro quadrático médio é 23,66 e corresponde a 2,6 vezes o erro quadrático médio $(9,11)$ obtido quando usamos o preditor $\hat{Q}_{i}^{(1)}$ calculado conforme a interpretação usual. Na realidade, o não enviesamento (em média) não é uma propriedade desejável neste caso, pois seria mais interessante que os preditores fossem não-enviesados para cada valor latente (ver discussão em Robinson (1991), por exemplo). Por esse motivo há interesse em saber se o preditor $\hat{Q}_{i}^{(1)}$ calculado conforme a interpretação usual apresenta erros quadráticos médios (EQM) menores em situações mais gerais. Essencialmente queremos avaliar qual dos dois preditores, (3.1) ou (3.2) comporta-se melhor na presença de variabilidade endógena heterocedástica.

\subsection{Simulação para $N=3$ e $n=2$}

A fim de comparar os EQM dos preditores (3.1) e (3.2) realizou-se um estudo de simulação ${ }^{2}$ com as seguintes etapas:

1) Definimos 36 cenários de simulação, mantendo fixos os valores latentes $(10,3,2)$ dos três indivíduos apresentados na Tabela 3.1 e modificando a relação (6 níveis) entre a média das variâncias dos erros de medida endógenos e a variância dos valores latentes e a distribuição dos erros de medida endógenos (6 distribuições). As variâncias dos erros de medida endógenos foram geradas tais que sua média correspondessem a um dos seguintes seis múltiplos de $\gamma^{2}=19,4,1,1 / 4,1 / 19$ e 1/99. Como consequência, a constante de encolhimento $k=\gamma^{2}\left(\gamma^{2}+\bar{\sigma}^{2}\right)^{-1}$ do preditor $\hat{Q}_{i}^{(2)}$ assume os valores $0,05,0,20,0,50,0,80,0,95$ e 0,99; em particular, $k=1$ corresponde à situação sem erro de medida endógeno. Para cada valor de $\bar{\sigma}^{2} / \gamma^{2}$ consideramos seis diferentes distribuições de probabilidade para os erros de medida endógenos: Normal, Uniforme, Beta simétrica, Beta assimétrica, Exponencial ou Gama.

2) Para cada um dos 36 cenários descritos acima, geramos 1.000 conjuntos de variâncias de erros de medida, $\sigma_{s}^{2}, s=1,2,3$, satisfazendo as relações indicadas.

3) Para cada uma das 36.000 situações descritas acima geramos 10.000 réplicas adicio-

\footnotetext{
${ }^{2} \mathrm{O}$ código escrito no programa $\mathrm{R}$ encontra-se no Apêndice $\mathrm{C}$
} 
nando os erros de medida em cada elemento das 24 possíveis amostras de tamanho $n=2$ da população finita.

4) Para cada uma das 36.000 situações descritas acima calculamos os dois preditores, seus viéses e seus respectivos EQM.

Em cada caso calculamos o quociente entre $\operatorname{EQM}\left[\hat{Q}_{i}^{(1)}\right]$ e $\operatorname{EQM}\left[\hat{Q}_{i}^{(2)}\right]$. Valores deste quociente maiores que 1 indicam que o EQM do preditor $\hat{Q}_{i}^{(1)}$ é maior que o EQM do preditor $\hat{Q}_{i}^{(2)}$. Quando o valor deste quociente está próximo de 1, os dois preditores produzem EQM muito próximos, quando está perto de zero o preditor $\hat{Q}_{i}^{(1)}$ produz EQM muito menor que o EQM produzido pelo preditor $\hat{Q}_{i}^{(2)}$. O coeficiente de variação das variâncias endógenas é utilizado como índice de heterocedasticidade. Quando esse índice está perto de zero, as variâncias endógenas estão próximas da variância média $\bar{\sigma}^{2}$, logo as constantes de encolhimento $k_{i}$ do preditor $\hat{Q}_{i}^{(1)}$ estão próximas da constante de encolhimento $k$ do preditor $\hat{Q}_{i}^{(2)}$. Este índice cresce quando as variâncias endógenas são mais dispersas. Também calculamos o número de situações em que o preditor $\hat{Q}_{i}^{(1)}$ tem menor EQM que o preditor $\hat{Q}_{i}^{(2)}$.

Tabela 3.4: Número de populações (num total de 1000) em que o preditor $\hat{Q}_{i}^{(1)}$ tem menor erro quadrático médio que o preditor $\hat{Q}_{i}^{(2)}$.

\begin{tabular}{c|c|c|c|c|c|c}
\hline $\bar{\sigma}^{2} / \gamma^{2}$ & Normal & Uniforme & Beta simétrica & Beta assimétrica & Exponencial & Gama \\
\hline 19 & 991 & 999 & 1000 & 995 & 992 & 992 \\
4 & 989 & 988 & 985 & 998 & 980 & 980 \\
1 & 892 & 911 & 905 & 997 & 903 & 899 \\
$1 / 4$ & 779 & 777 & 749 & 991 & 778 & 762 \\
$1 / 19$ & 690 & 706 & 738 & 991 & 737 & 756 \\
$1 / 99$ & 689 & 700 & 718 & 989 & 712 & 713 \\
\hline Total & 5030 & 5081 & 5095 & 5961 & 5102 & 5102 \\
\hline
\end{tabular}

O estudo de simulação mostrou que o preditor $\hat{Q}_{i}^{(1)}$ tem erro quadrático médio menor que o preditor $\hat{Q}_{i}^{(2)}$ em aproximadamente $85 \%$ das situações simuladas quando a distribuição do erro de medida endógeno é Normal, Uniforme, Beta simétrica, Exponencial e Gama. Quando o erro de medida endógeno tem distribuição Beta assimétrica esse valor é aproximadamente $99 \%$ (veja Tabela 3.4 ). Nos casos em que o preditor $\hat{Q}_{i}^{(1)}$ tem EQM 
Figura 3.1: Boxplot para quocientes $\operatorname{EQM}\left[\hat{Q}_{i}^{(1)}\right] / \operatorname{EMQ}\left[\hat{Q}_{i}^{(2)}\right]$ sob erros de medida endóge$\operatorname{nos}$ para $N=3$ e $n=2$.
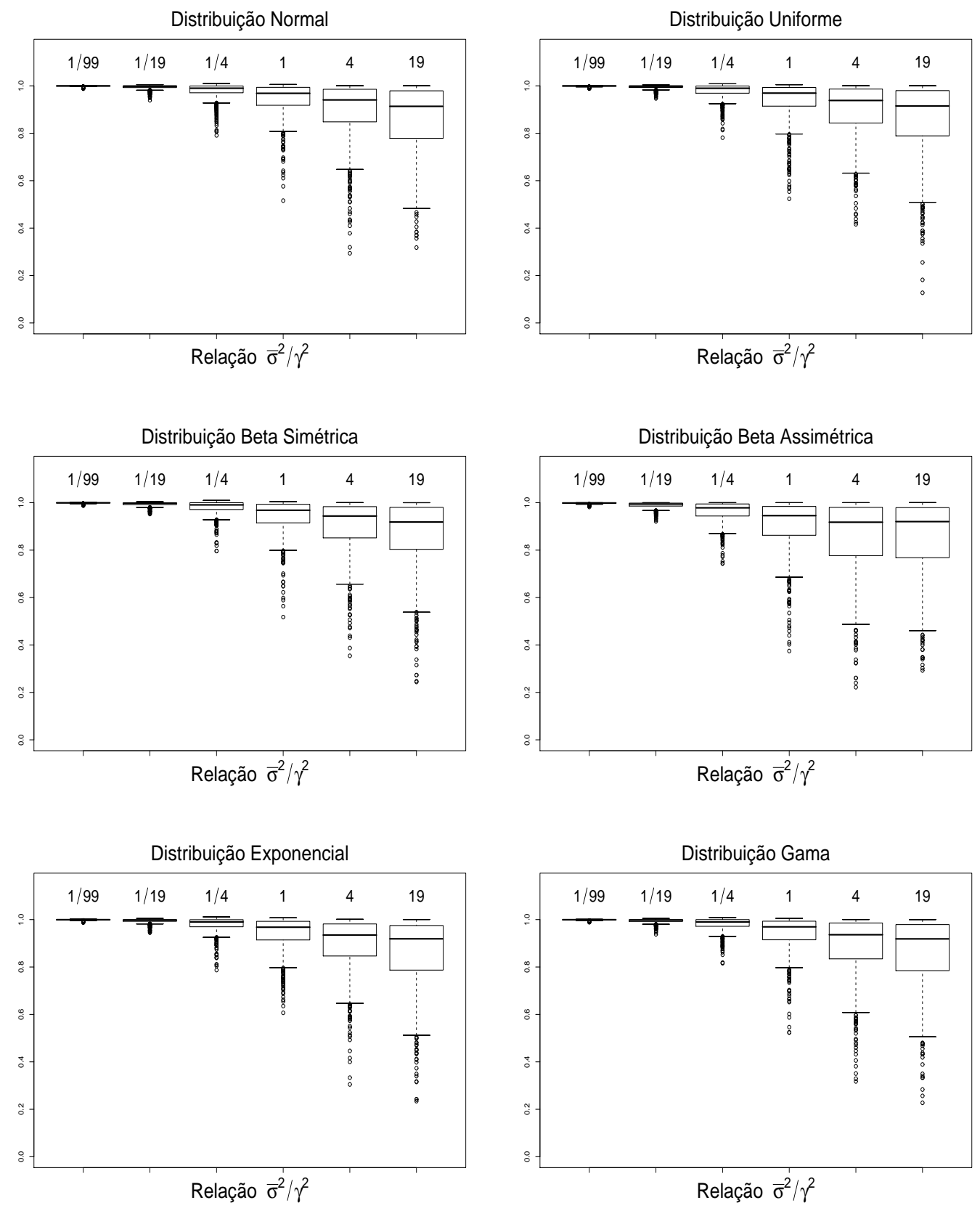
Figura 3.2: Quocientes $\operatorname{EQM}\left[\hat{Q}_{i}^{(1)}\right] / \operatorname{EMQ}\left[\hat{Q}_{i}^{(2)}\right]$ sob erros de medida endógenos com distribuição Uniforme para $N=3$ e $n=2$.
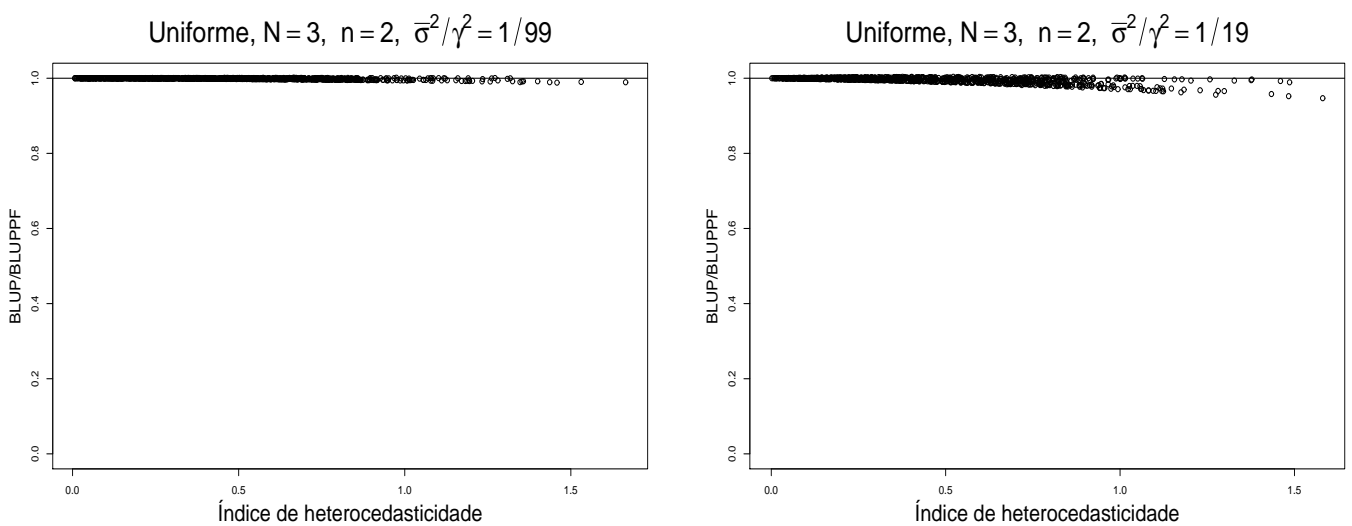

Uniforme, $\mathrm{N}=3, \mathrm{n}=2, \bar{\sigma}^{2} / \gamma^{2}=1 / 4$

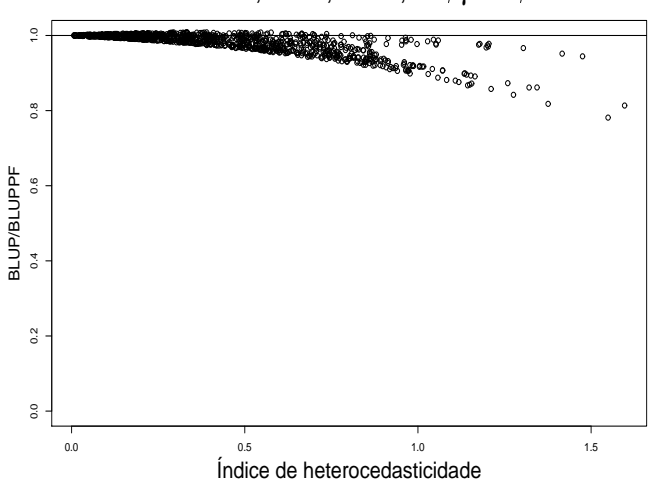

Uniforme, $\mathrm{N}=3, \mathrm{n}=2, \bar{\sigma}^{2} / \mathrm{r}^{2}=1$

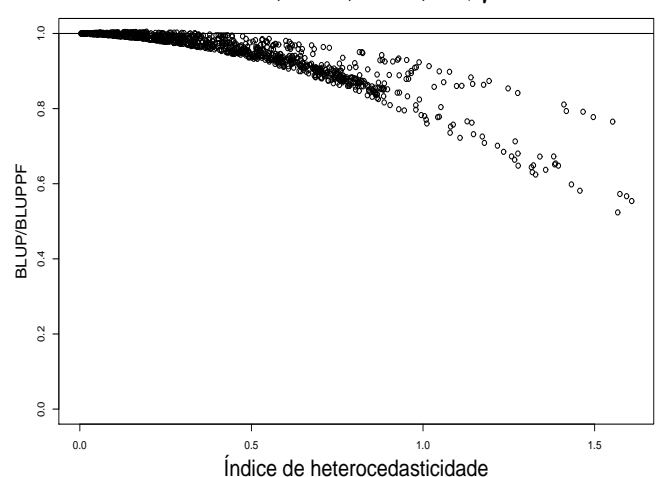

Uniforme, $\mathrm{N}=3, \mathrm{n}=2, \bar{\sigma}^{2} / \gamma^{2}=4$
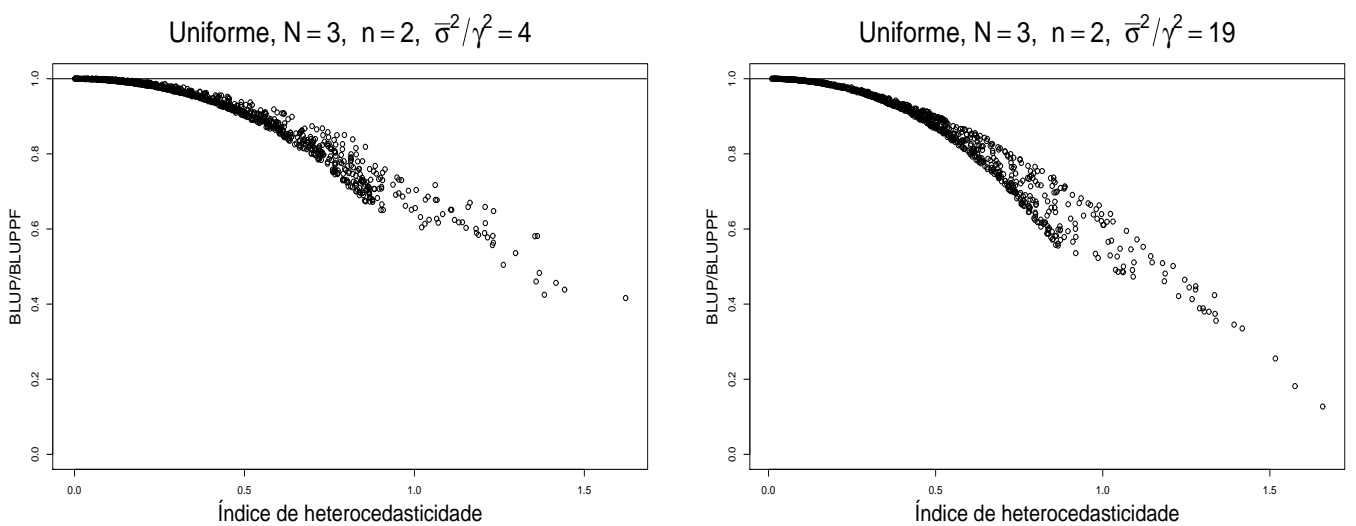
Tabela 3.5: Estatísticas descritivas para os quocientes $\operatorname{EQM}\left[\hat{Q}_{i}^{(1)}\right] / \operatorname{EMQ}\left[\hat{Q}_{i}^{(2)}\right]$ sob erros de medida endógenos com distribuição Normal para $N=3$ e $n=2$.

\begin{tabular}{c|c|c|c|c|c|c}
\hline $\bar{\sigma}^{2} / \gamma^{2}$ & Mínimo & $Q_{1}$ & Mediana & Média & $Q_{3}$ & Máximo \\
\hline 19 & 0,318 & 0,779 & 0,914 & 0,865 & 0,979 & 1,000 \\
4 & 0,294 & 0,848 & 0,941 & 0,899 & 0,985 & 1,000 \\
1 & 0,516 & 0,918 & 0,969 & 0,947 & 0,994 & 1,006 \\
$1 / 4$ & 0,791 & 0,971 & 0,990 & 0,980 & 0,999 & 1,010 \\
$1 / 19$ & 0,939 & 0,993 & 0,998 & 0,995 & 1,000 & 1,005 \\
$1 / 99$ & 0,988 & 0,999 & 1,000 & 0,999 & 1,000 & 1,001 \\
\hline
\end{tabular}

maior que o EQM do preditor $\hat{Q}_{i}^{(2)}$, por exemplo, quando a distribuição do erro de medida endógeno é Normal, a diferença não foi superior ao $1 \%$ (veja Tabela (3.5). Resultados similares podem ser verificados para as outras distribuições dos erros de medida endógenos (veja Tabelas C.1-C.5 no Apêndice C.2).

Independentemente da forma das distribuições dos erros de medida, quanto menor o quociente entre a média das variâncias dos erros de medida endógenos e a variância dos valores latentes $\left(\bar{\sigma}^{2} / \gamma^{2}\right)$, menores são as diferenças entre os EQM dos preditores $\hat{Q}_{i}^{(1)}$ e $\hat{Q}_{i}^{(2)}$ (veja Figura 3.1); quando essa relação é pequena os dois preditores tem o EQM muito próximos (veja Figura 3.2 e Figuras C.1-C.5 no Apêndice C.2). Na realidade, quanto maior seja a relação $\bar{\sigma}^{2} / \gamma^{2}$ o preditor $\hat{Q}_{i}^{(1)}$ produz estimativas dos valores latentes menos enviesados para cada indivíduo.

Apesar de o preditor obtido a partir do modelo misto usual $\left(\hat{Q}_{i}^{(1)}\right.$, utilizado conforme a interpretação usual) ser enviesado em média (veja Figura 3.3), ele apresenta um erro quadrático médio menor que o do preditor $\left(\hat{Q}_{i}^{(2)}\right)$ obtido a partir do MMPF. Quando $\hat{Q}_{i}^{(1)}$ não gera o menor erro quadrático médio, os dois preditores geram EQM muito próximos.

\subsection{Simulação para outros valores de $N$ e $n$}

Na maioria das situações simuladas para $N=3$ e $n=2$ o preditor $\hat{Q}_{i}^{(1)}$ baseado no modelo misto usual e calculado conforme a interpretação usual apresenta erros quadráticos médios (EQM) menores que o preditor $\hat{Q}_{i}^{(2)}$ obtido a partir do modelo misto para populações finitas. A seguir avaliamos qual dos dois preditores, comporta-se melhor em 
Figura 3.3: Boxplot para o viés da média populacional quando usamos o preditor $\hat{Q}_{i}^{(1)}$ para $N=3$ e $n=2$.
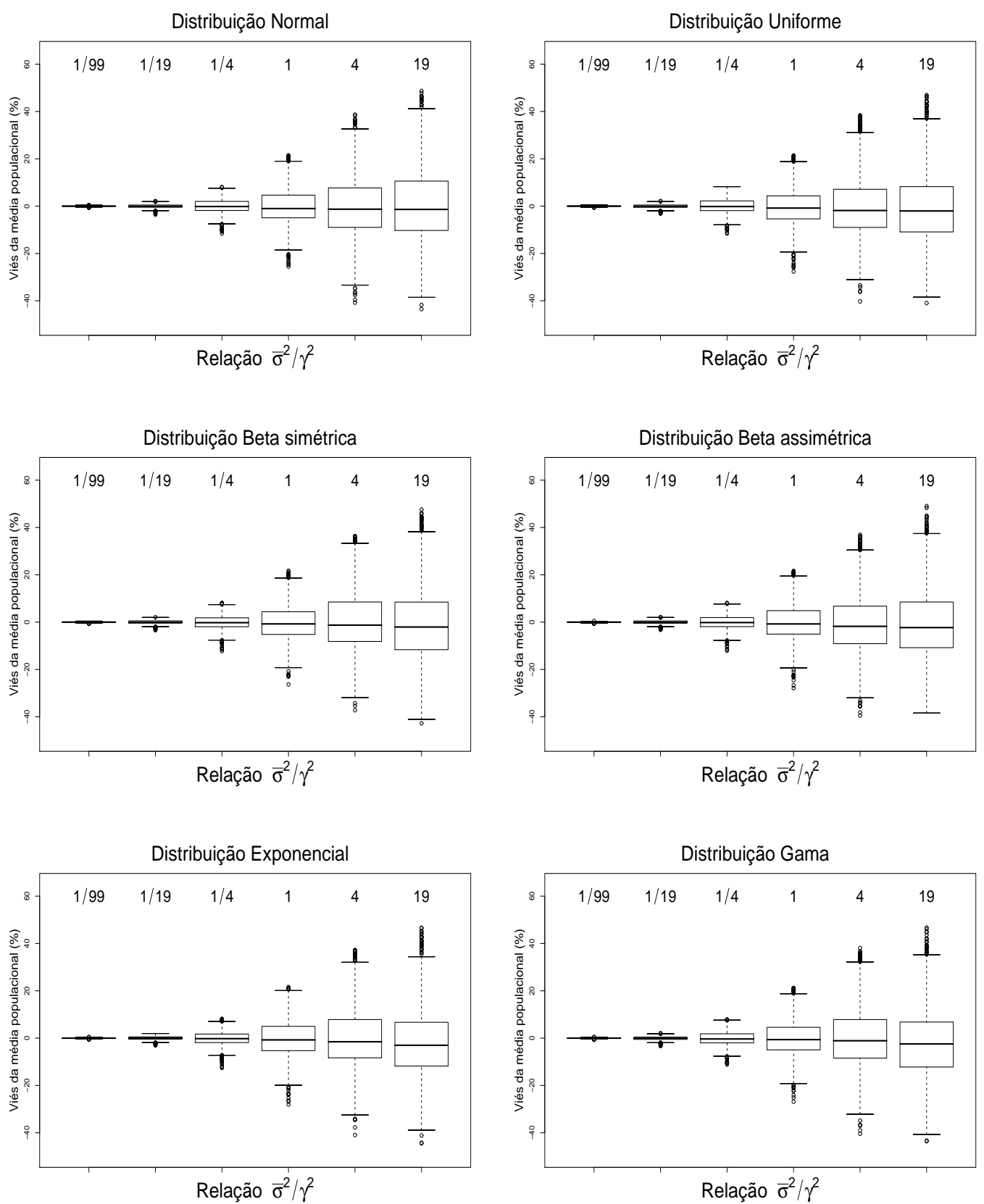
termos de EQM na presença de variabilidade endógena heterocedástica em situações mais gerais com relação ao tamanho de população, ao tamanho amostral e à distribuição dos valores latentes.

Consideramos 972 situações diferindo com relação ao tamanho populacional (3 tamanhos), ao tamanho amostral (3 tamanhos amostrais para cada tamanho populacional), à forma da distribuição dos valores latentes (3 distribuições), ao quociente entre a média das variâncias dos erros de medida endógenos e a variância dos valores latentes (6 níveis) e a distribuição dos erros de medida endógenos (6 distribuições), descritas da seguinte forma.

1) Definimos 3 tamanhos para a população finita, a saber, $N=50$, 100 e 300 .

2) Para cada tamanho populacional usamos três tamanhos amostrais diferentes, ou seja, $n=\{5,10,25\},\{10,30,50\}$ e $\{30,50,100\}$, respectivamente.

3) Consideramos 3 tipos de distribuição para gerar os valores latentes das unidades da população finita, fixando a média populacional em 180: uma distribuição uniforme no intervalo $(60,300)$, uma distribuição assimétrica à esquerda usando a transformação $Y=252 X, \operatorname{com} X \sim \operatorname{Beta}(5,2)$ e $\mathbb{E}(X)=5 / 7$, e uma distribuição assimétrica à direita usando a transformação $Y=100+280 X$, com $X \sim \operatorname{Beta}(2,5)$ e $\mathbb{E}(X)=2 / 7$.

4) Para cada uma das 27 combinações de tamanho de população, tamanho da amostra e forma da distribuição dos valores latentes, definimos 36 cenários de simulação, mantendo fixos os valores latentes dos indivíduos da população e modificando o quociente entre a média das variâncias dos erros de medida endógenos e a variância dos valores latentes e a distribuição dos erros de medida endógenos.

5) Para cada uma das 972 situações descritas acima, geramos 500 conjuntos de variâncias de erros de medida endógenos, $\sigma_{s}^{2}, s=1,2, \ldots, N$, satisfazendo as condições acima.

6) Para cada uma das $972 \times 500=486.000$ situações descritas acima, geramos 1.000 amostras independentes de tamanho $n$ via amostragem aleatória simples. Geramos 100 réplicas adicionando os erros de medida endógenos em cada elemento das 1.000 amostras de tamanho $n$.

7) Para cada uma das 486.000 situações descritas acima calculamos os dois preditores, seus viéses e seus respectivos EQM. 
Tabela 3.6: Número de populações em que o preditor $\hat{Q}_{i}^{(1)}$ tem menor erro quadrático médio que o preditor $\hat{Q}_{i}^{(2)}$ de um total de 500 populações simuladas com valores latentes seguindo uma distribuição uniforme.

\begin{tabular}{|c|c|c|c|c|c|c|c|c|}
\hline & & & \multicolumn{6}{|c|}{ Distribuição dos erros de medida endógenos } \\
\hline$N$ & $n$ & $\bar{\sigma}^{2} / \gamma^{2}$ & Normal & Uniforme & Beta Simétrica & Beta Assimétrica & Exponencial & Gama \\
\hline \multirow{6}{*}{50} & \multirow{6}{*}{5} & 19 & 500 & 500 & 500 & 500 & 500 & 500 \\
\hline & & 4 & 500 & 500 & 500 & 500 & 499 & 500 \\
\hline & & 1 & 500 & 494 & 498 & 496 & 498 & 498 \\
\hline & & $1 / 4$ & 495 & 494 & 493 & 494 & 497 & 486 \\
\hline & & $1 / 19$ & 480 & 481 & 478 & 475 & 476 & 465 \\
\hline & & $1 / 99$ & 437 & 452 & 444 & 450 & 446 & 436 \\
\hline \multirow{6}{*}{50} & \multirow{6}{*}{10} & 19 & 500 & 500 & 500 & 500 & 500 & 500 \\
\hline & & 4 & 500 & 500 & 500 & 500 & 500 & 500 \\
\hline & & 1 & 498 & 497 & 496 & 498 & 499 & 496 \\
\hline & & $1 / 4$ & 490 & 489 & 492 & 494 & 492 & 487 \\
\hline & & $1 / 19$ & 481 & 478 & 484 & 483 & 470 & 474 \\
\hline & & $1 / 99$ & 458 & 460 & 453 & 452 & 460 & 464 \\
\hline \multirow{6}{*}{50} & \multirow{6}{*}{25} & 19 & 500 & 500 & 500 & 500 & 500 & 500 \\
\hline & & 4 & 500 & 499 & 499 & 499 & 498 & 500 \\
\hline & & 1 & 491 & 497 & 497 & 495 & 494 & 499 \\
\hline & & $1 / 4$ & 488 & 488 & 487 & 496 & 493 & 485 \\
\hline & & $1 / 19$ & 489 & 480 & 486 & 475 & 480 & 482 \\
\hline & & $1 / 99$ & 468 & 471 & 461 & 461 & 462 & 458 \\
\hline \multirow{6}{*}{100} & \multirow{6}{*}{10} & 19 & 500 & 500 & 500 & 500 & 500 & 500 \\
\hline & & 4 & 500 & 500 & 500 & 500 & 500 & 500 \\
\hline & & 1 & 500 & 500 & 499 & 500 & 498 & 498 \\
\hline & & $1 / 4$ & 500 & 499 & 498 & 494 & 496 & 499 \\
\hline & & $1 / 19$ & 495 & 488 & 493 & 492 & 491 & 497 \\
\hline & & $1 / 99$ & 468 & 470 & 474 & 474 & 475 & 474 \\
\hline \multirow{6}{*}{100} & \multirow{6}{*}{30} & 19 & 500 & 500 & 500 & 500 & 500 & 500 \\
\hline & & 4 & 500 & 500 & 500 & 500 & 500 & 500 \\
\hline & & 1 & 500 & 498 & 497 & 499 & 498 & 498 \\
\hline & & $1 / 4$ & 498 & 498 & 500 & 499 & 499 & 499 \\
\hline & & $1 / 19$ & 499 & 495 & 496 & 495 & 495 & 490 \\
\hline & & $1 / 99$ & 483 & 482 & 486 & 484 & 485 & 489 \\
\hline \multirow{6}{*}{100} & \multirow{6}{*}{50} & 19 & 500 & 500 & 500 & 500 & 500 & 500 \\
\hline & & 4 & 500 & 500 & 500 & 500 & 500 & 500 \\
\hline & & 1 & 499 & 499 & 498 & 498 & 499 & 500 \\
\hline & & $1 / 4$ & 499 & 499 & 499 & 497 & 498 & 499 \\
\hline & & $1 / 19$ & 498 & 493 & 493 & 498 & 492 & 497 \\
\hline & & $1 / 99$ & 488 & 490 & 487 & 490 & 485 & 489 \\
\hline \multirow{6}{*}{300} & \multirow{6}{*}{30} & 19 & 500 & 500 & 500 & 500 & 500 & 500 \\
\hline & & 4 & 500 & 500 & 500 & 500 & 500 & 500 \\
\hline & & 1 & 500 & 500 & 500 & 500 & 500 & 500 \\
\hline & & $1 / 4$ & 500 & 500 & 500 & 500 & 500 & 500 \\
\hline & & $1 / 19$ & 500 & 500 & 499 & 500 & 499 & 500 \\
\hline & & $1 / 99$ & 496 & 497 & 496 & 495 & 496 & 497 \\
\hline \multirow{6}{*}{300} & \multirow{6}{*}{50} & 19 & 500 & 500 & 500 & 500 & 500 & 500 \\
\hline & & 4 & 500 & 500 & 500 & 500 & 500 & 500 \\
\hline & & 1 & 500 & 500 & 500 & 500 & 499 & 500 \\
\hline & & $1 / 4$ & 500 & 500 & 499 & 500 & 500 & 500 \\
\hline & & $1 / 19$ & 500 & 500 & 500 & 500 & 500 & 499 \\
\hline & & $1 / 99$ & 500 & 498 & 499 & 498 & 500 & 500 \\
\hline & & 19 & 500 & 500 & 500 & 500 & 500 & 500 \\
\hline & & 4 & 500 & 499 & 500 & 500 & 500 & 500 \\
\hline 300 & 100 & 1 & 500 & 500 & 500 & 500 & 500 & 500 \\
\hline & & $1 / 4$ & 500 & 500 & 500 & 500 & 500 & 500 \\
\hline & & $1 / 19$ & 500 & 500 & 499 & 500 & 500 & 500 \\
\hline & & $1 / 99$ & 500 & 500 & 500 & 500 & 499 & 500 \\
\hline
\end{tabular}


Tabela 3.7: Valor máximo do quociente $\operatorname{EQM}\left[\hat{Q}_{i}^{(1)}\right] / \operatorname{EMQ}\left[\hat{Q}_{i}^{(2)}\right]$ com valores latentes seguindo uma distribuição uniforme.

\begin{tabular}{|c|c|c|c|c|c|c|c|c|}
\hline & & & \multicolumn{6}{|c|}{ Distribuição dos erros de medida endógenos } \\
\hline$N$ & $n$ & $\bar{\sigma}^{2} / \gamma^{2}$ & Normal & Uniforme & Beta Simétrica & Beta Assimétrica & Exponencial & Gama \\
\hline \multirow{6}{*}{50} & \multirow{6}{*}{5} & 19 & 0.977 & 0.974 & 0.967 & 0.974 & 0.984 & 0.986 \\
\hline & & 4 & 0.980 & 0.984 & 0.992 & 0.987 & 1.002 & 0.995 \\
\hline & & 1 & 0.996 & 1.011 & 1.008 & 1.002 & 1.019 & 1.024 \\
\hline & & $1 / 4$ & 1.015 & 1.005 & 1.028 & 1.011 & 1.006 & 1.032 \\
\hline & & $1 / 19$ & 1.011 & 1.027 & 1.025 & 1.025 & 1.166 & 1.036 \\
\hline & & $1 / 99$ & 1.081 & 1.035 & 1.026 & 1.036 & 1.068 & 1.020 \\
\hline \multirow{6}{*}{50} & \multirow{6}{*}{10} & 19 & 0.976 & 0.985 & 0.985 & 0.953 & 0.970 & 0.971 \\
\hline & & 4 & 0.992 & 0.988 & 0.996 & 0.985 & 0.991 & 0.991 \\
\hline & & 1 & 1.008 & 1.030 & 1.029 & 1.020 & 1.007 & 1.022 \\
\hline & & $1 / 4$ & 1.012 & 1.012 & 1.027 & 1.013 & 1.014 & 1.015 \\
\hline & & $1 / 19$ & 1.030 & 1.023 & 1.016 & 1.012 & 1.013 & 1.018 \\
\hline & & $1 / 99$ & 1.025 & 1.023 & 1.036 & 1.046 & 1.033 & 1.021 \\
\hline \multirow{6}{*}{50} & \multirow{6}{*}{25} & 19 & 0.982 & 0.979 & 0.982 & 0.977 & 0.989 & 0.991 \\
\hline & & 4 & 0.998 & 1.004 & 1.001 & 1.002 & 1.018 & 1.000 \\
\hline & & 1 & 1.025 & 1.009 & 1.015 & 1.018 & 1.018 & 1.009 \\
\hline & & $1 / 4$ & 1.045 & 1.020 & 1.018 & 1.036 & 1.010 & 1.027 \\
\hline & & $1 / 19$ & 1.046 & 1.072 & 1.020 & 1.033 & 1.033 & 1.030 \\
\hline & & $1 / 99$ & 1.022 & 1.044 & 1.017 & 1.033 & 1.028 & 1.051 \\
\hline \multirow{6}{*}{100} & \multirow{6}{*}{10} & 19 & 0.966 & 0.977 & 0.971 & 0.967 & 0.981 & 0.963 \\
\hline & & 4 & 0.983 & 0.985 & 0.979 & 0.980 & 0.994 & 0.986 \\
\hline & & 1 & 0.997 & 0.999 & 1.011 & 0.996 & 1.010 & 1.006 \\
\hline & & $1 / 4$ & 0.997 & 1.041 & 1.010 & 1.013 & 1.009 & 1.007 \\
\hline & & $1 / 19$ & 1.008 & 1.071 & 1.007 & 1.013 & 1.005 & 1.051 \\
\hline & & $1 / 99$ & 1.017 & 1.066 & 1.008 & 1.008 & 1.022 & 1.014 \\
\hline \multirow{6}{*}{100} & \multirow{6}{*}{30} & 19 & 0.980 & 0.984 & 0.978 & 0.976 & 0.976 & 0.984 \\
\hline & & 4 & 0.996 & 0.997 & 0.994 & 0.997 & 0.994 & 0.986 \\
\hline & & 1 & 0.999 & 1.014 & 1.020 & 1.013 & 1.017 & 1.011 \\
\hline & & $1 / 4$ & 1.001 & 1.001 & 0.998 & 1.001 & 1.001 & 1.001 \\
\hline & & $1 / 19$ & 1.002 & 1.016 & 1.004 & 1.004 & 1.004 & 1.009 \\
\hline & & $1 / 99$ & 1.003 & 1.014 & 1.017 & 1.004 & 1.026 & 1.004 \\
\hline \multirow{6}{*}{100} & \multirow{6}{*}{50} & 19 & 0.982 & 0.980 & 0.992 & 0.992 & 0.981 & 0.988 \\
\hline & & 4 & 0.991 & 0.997 & 0.996 & 0.992 & 0.995 & 0.999 \\
\hline & & 1 & 1.000 & 1.005 & 1.003 & 1.012 & 1.001 & 0.990 \\
\hline & & $1 / 4$ & 1.004 & 1.005 & 1.001 & 1.002 & 1.008 & 1.002 \\
\hline & & $1 / 19$ & 1.001 & 1.004 & 1.015 & 1.003 & 1.002 & 1.005 \\
\hline & & $1 / 99$ & 1.006 & 1.004 & 1.003 & 1.020 & 1.010 & 1.003 \\
\hline \multirow{6}{*}{300} & \multirow{6}{*}{30} & 19 & 0.980 & 0.984 & 0.989 & 0.979 & 0.982 & 0.977 \\
\hline & & 4 & 0.989 & 0.985 & 0.986 & 0.987 & 0.988 & 0.989 \\
\hline & & 1 & 0.990 & 0.990 & 0.992 & 0.989 & 0.988 & 0.986 \\
\hline & & $1 / 4$ & 0.996 & 0.995 & 0.995 & 0.992 & 0.994 & 0.996 \\
\hline & & $1 / 19$ & 1.000 & 0.999 & 1.000 & 0.998 & 1.000 & 0.999 \\
\hline & & $1 / 99$ & 1.000 & 1.000 & 1.000 & 1.013 & 1.001 & 1.000 \\
\hline \multirow{6}{*}{300} & \multirow{6}{*}{50} & 19 & 0.992 & 0.986 & 0.986 & 0.981 & 0.986 & 0.988 \\
\hline & & 4 & 0.993 & 0.990 & 0.992 & 0.990 & 0.991 & 0.991 \\
\hline & & 1 & 0.990 & 0.987 & 0.985 & 0.988 & 1.001 & 0.993 \\
\hline & & $1 / 4$ & 0.996 & 0.999 & 1.001 & 0.999 & 0.992 & 0.993 \\
\hline & & $1 / 19$ & 0.999 & 0.999 & 0.998 & 0.998 & 0.997 & 1.000 \\
\hline & & $1 / 99$ & 1.000 & 1.000 & 1.000 & 1.002 & 1.000 & 1.000 \\
\hline & & 19 & 0.989 & 0.986 & 0.993 & 0.990 & 0.988 & 0.991 \\
\hline & & 4 & 0.993 & 0.989 & 0.996 & 0.988 & 0.991 & 0.990 \\
\hline 300 & 100 & 1 & 0.991 & 0.999 & 0.988 & 0.990 & 0.994 & 0.990 \\
\hline & & $1 / 4$ & 0.992 & 0.997 & 0.997 & 0.991 & 0.995 & 0.996 \\
\hline & & $1 / 19$ & 0.999 & 0.999 & 0.999 & 0.999 & 0.999 & 0.997 \\
\hline & & $1 / 99$ & 1.000 & 1.000 & 1.000 & 1.000 & 1.002 & 1.000 \\
\hline
\end{tabular}


Para cada cenário simulado, calculamos o quociente entre $E Q M\left[\hat{Q}_{i}^{(1)}\right]$ e $E M Q\left[\hat{Q}_{i}^{(2)}\right]$, o índice de heterocedasticidade das variâncias endógenas e o número de situações em que o preditor $\hat{Q}_{i}^{(1)}$ tem menor EQM que o preditor $\hat{Q}_{i}^{(2)}$. Foi usado o programa $\mathrm{R}$ para as simulações e o tempo de procesamento de cada cénario foi em média de 4 horas, usando computadores com processador Intel Core Duo T2250 de $1.73 \mathrm{GHz} / 2 \mathrm{MB}$ de Cache.

O estudo de simulação mostrou que o preditor $\hat{Q}_{i}^{(1)}$ tem erro quadrático médio menor que o preditor $\hat{Q}_{i}^{(2)}$ em aproximadamente $96 \%$ das 486.000 situações simuladas; $99 \%$ das 162.000 situações quando os valores latentes foram gerados com distribuição uniforme, 92\% das 162.000 situações quando os valores latentes foram gerados com distribuição assimétrica à esquerda e 97\% das 162.000 situações quando os valores latentes foram gerados com distribuição assimétrica à direita (veja Tabela 3.6 e Tabelas D.1 e D.2 no Apêndice D).

Nos casos em que o preditor $\hat{Q}_{i}^{(1)}$ tem o EQM maior que o EQM do preditor $\hat{Q}_{i}^{(2)}$, em média, essa diferença não foi superior a $2 \%$ quando os valores latentes são gerados com distribuição uniforme, nem superior a $7 \%$ quando os valores latentes foram gerados com distribuição assimétrica (veja Tabela 3.7 e Tabelas D.3 e D.4 no Apêndice D).

Independentemente da forma das distribuições dos erros de medida endógenos e dos valores latentes, quanto maior é o quociente entre a média das variâncias dos erros de medida endógenos e a variância dos valores latentes, maiores são as diferenças entre os EQM de $\hat{Q}_{i}^{(1)}$ e $\hat{Q}_{i}^{(2)}$ (veja Figuras E.1-J.9 nos Apêndices E-J). Quando essa relação é pequena $\left(\bar{\sigma}^{2} / \gamma^{2}=1 / 19\right.$ ou $\left.\bar{\sigma}^{2} / \gamma^{2}=1 / 99\right)$ os dois preditores tem os EQM muito próximos.

O número de situações em que o preditor $\hat{Q}_{i}^{(1)}$ apresenta menor EQM com relação ao preditor $\hat{Q}_{i}^{(2)}$ é maior quando os valores latentes foram gerados com distribuição uniforme do que quando foram gerados com distribuição assimétrica à esquerda ou assimétrica à direita.

Quanto maior é o tamanho da população finita, o preditor $\hat{Q}_{i}^{(1)}$ apresenta menores EQM com relação ao preditor $\hat{Q}_{i}^{(2)}$. Quanto maior é o tamanho da amostra com relação ao tamanho da população, o preditor $\hat{Q}_{i}^{(1)}$ apresenta menores EQM com relação ao preditor $\hat{Q}_{i}^{(2)}$.

Independentemente do tamanho da população, do tamanho da amostra, da forma das distribuições dos erros de medida endógenos e dos valores latentes, do quociente entre a média das variâncias dos erros de medida endógenos e a variância dos valores latentes e 
apesar de o preditor obtido a partir do modelo misto usual $\left(\hat{Q}_{i}^{(1)}\right.$, utilizado conforme a interpretação usual) ser enviesado em média, ele apresenta menor erro quadrático médio que o preditor $\left(\hat{Q}_{i}^{(2)}\right)$ obtido a partir do MMPF na maioria das situações simuladas. Quando $\hat{Q}_{i}^{(1)}$ não gera o menor erro quadrático médio, em média, essa diferença é menor do que $7 \%$. 


\section{Capítulo 4}

\section{Aspectos computacionais}

Em aplicações práticas, é possível ajustar modelos mistos a dados sujeitos a erros de medida endógenos e exógenos, homocedásticos ou heterocedásticos, utilizando rotinas desenvolvidas com base no modelo misto usual e implementadas nos Software estatísticos como SAS (proc MIXED) e R (nlme). Neste capítulo, apresentamos códigos computacionais desenvolvidos com essa finalidade.

O BLUP do valor latente da $i$-ésima unidade selecionada na amostra, obtido a partir do modelo misto para populações finitas com presença simultanea dos dois erros de medida (2.20), é

$$
\hat{Y}_{i}=\overline{\bar{Y}}+\frac{\gamma^{2}}{\gamma^{2}+\bar{\sigma}^{2}+\tilde{\sigma}_{i}^{2}}\left(\tilde{Y}_{i}-\overline{\bar{Y}}\right),
$$

em que $\tilde{\sigma}_{i}^{2}$ é a variância do erro de medida exógeno referente à $i$-ésima condição de medida e $\bar{\sigma}^{2}$ é a média das variâncias individuais dos erros de medida endógenos. Quando não há variabilidade endógena, isto é, quando $\bar{\sigma}^{2}=0$, o preditor é

$$
\hat{Y}_{i}=\overline{\bar{Y}}+\frac{\gamma^{2}}{\gamma^{2}+\tilde{\sigma}_{i}^{2}}\left(\tilde{Y}_{i}-\overline{\bar{Y}}\right) .
$$

Quando não há variabilidade exógena, isto é, quando $\tilde{\sigma}_{i}^{2}=0, i=1, \ldots, n$, o preditor é

$$
\hat{Y}_{i}=\overline{\bar{Y}}+\frac{\gamma^{2}}{\gamma^{2}+\bar{\sigma}^{2}}\left(\tilde{Y}_{i}-\overline{\bar{Y}}\right) .
$$

O modelo misto usual para a $k$-ésima medida da $i$-ésima unidade selecionada na amostra é escrito como

$$
Y_{i k}=\mu+B_{i}+E_{i k}, \quad i=1, \ldots, n, \quad k=1, \ldots, n_{i}, \quad B \stackrel{i i d}{\sim} N\left(0, \gamma^{2}\right)
$$


em que $E_{i k}$ é o erro de medida. O BLUP de $Y_{i}=\mu+B_{i}$ depende da estrutura de covariância proposta para o modelo (4.4); no caso de erros de medida heterocedásticos, $E_{i k} \stackrel{i i d}{\sim} N\left(0, \sigma_{i}^{2}\right)$ e no caso de erros de medida homocedásticos, $E_{i k} \stackrel{i i d}{\sim} N\left(0, \sigma^{2}\right)$. Os preditores de $Y_{i}=\mu+B_{i}$ sob esse modelo têm a forma (4.2) e (4.3) respectivamente. Nesse contexto é possível adaptar as sub-rotinas computacionais disponíveis nos pacotes destinados a análise estatística a fim de acomodar diferentes características de erros de medida endógenos e exógenos.

Como ilustração, consideramos o conjunto de dados apresentado no Capítulo 1 . Na Tabela 4.1 apresentamos as médias dos níveis de colesterol de cada indivíduo observados nos quatro trimestres e as tomamos como sendo o valor latente de cada indivíduo; as variâncias correspondentes, calculadas como $\sigma_{s}^{2}=\frac{1}{3} \sum_{q=1}^{4}\left(y_{s q}-y_{s}\right)^{2}, s=1, \ldots, 13$, são consideradas como as verdadeiras variâncias dos erros endógenos.

Tabela 4.1: Valores latentes e variâncias endógenas do nível de colesterol.

\begin{tabular}{c|c|c}
\hline Indivíduo & Valor latente & Variância endógena \\
$s$ & $y_{s}$ & $\sigma_{s}^{2}$ \\
\hline 1 & 242,1 & 3545,1 \\
2 & 263,2 & 1250,1 \\
3 & 154,5 & 1932,9 \\
4 & 232,6 & 3083,2 \\
5 & 202,2 & 1299,4 \\
6 & 280,4 & 1013,8 \\
7 & 268,4 & 2216,9 \\
8 & 198,4 & 1815,2 \\
9 & 303,4 & 5222,3 \\
10 & 237,7 & 548,9 \\
11 & 141,8 & 1099,4 \\
12 & 215,3 & 639,1 \\
13 & 128,8 & 207,8 \\
\hline
\end{tabular}

Admitimos que os diferentes examinadores correspondem a diferentes condições de avaliação e consequentemente, que os erros de medida associados a eles são erros de medida exógenos. As variâncias correspondentes são aquelas apresentadas na Tabela 1.4. Pode-se notar que tanto os erros de medida endógenos quanto os erros de medida exógenos são heterocedásticos. A variância populacional dos valores latentes é $\gamma^{2}=2939,9$ e a média 
das variâncias endógenas é $\bar{\sigma}^{2}=1836,5$.

\subsection{Modelos com erros de medida endógenos apenas}

Os preditores obtidos sob os modelos mistos usuais e MMPF são diferentes na presença de erros de medida endógenos heterocedásticos. No entanto, o preditor obtido sob o MMPF tem uma forma semelhante àquela do preditor obtido sob o modelo misto usual com erros de medida homocedásticos.

Podemos estimar as duas variâncias do modelo (4.3), $\gamma^{2}$ e $\bar{\sigma}^{2}$, e calcular os BLUP para os valores latentes correspondentes aos 13 indivíduos usando o seguinte código escrito no PROC MIXED do SAS ou com a função lme da sub-rotina nlme do R.

\section{- Código SAS}

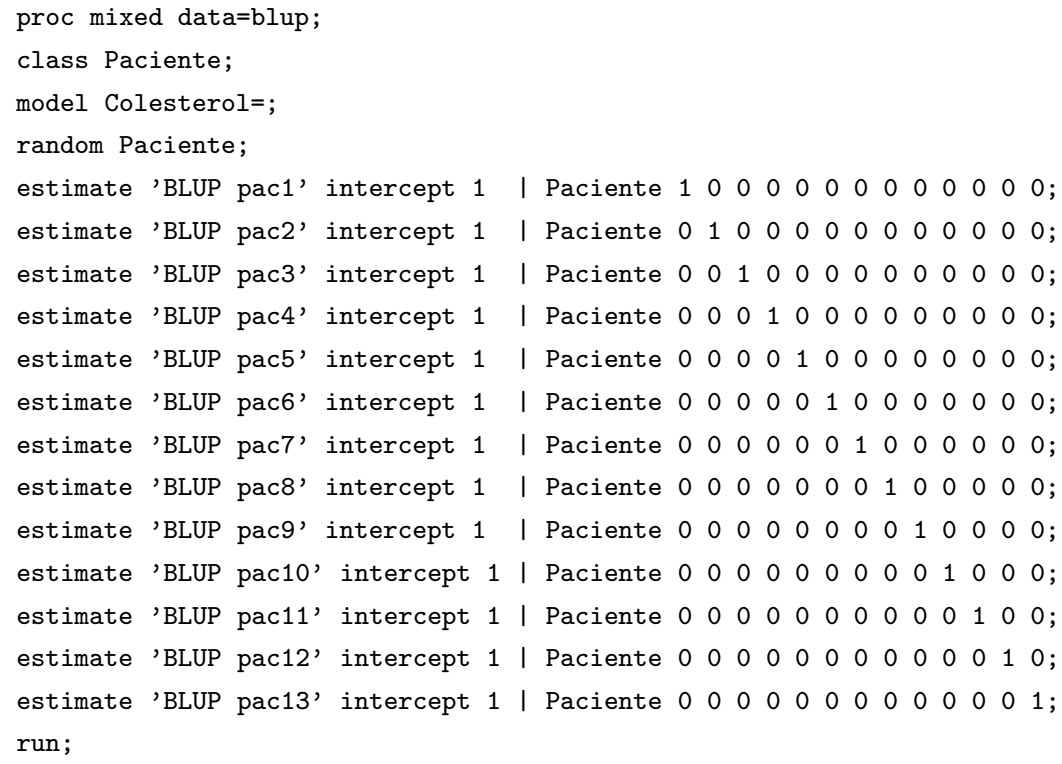

\section{- Código R}

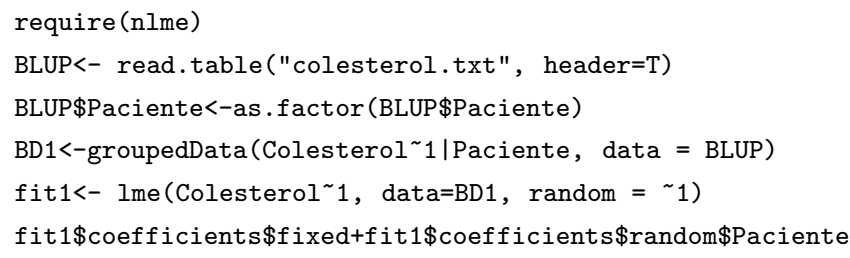


Os dados: a partir dos valores latentes e das variâncias endógenas apresentadas na Tabela 4.1 geramos um conjunto de 156 observações (13 grupos de 12 observações cada). Cada grupo de 12 observações foi obtido da seguinte forma: dividimos o intervalo $[0,1]$ em 13 intervalos de igual tamanho e obtivemos os percentis da distribuição normal correspondentes ao limite superior de cada intervalo, transformamos esses valores tal que sua média coincidisse com o valor latente, $y_{s}$, e sua variância com a variância endógena correspondente, $\sigma_{s}^{2}, s=1,2, \ldots, 13$.

O conjunto de dados no SAS deve ser apresentado em variáveis e observações. Para criar as variáveis usamos a palavra INPUT, aqui as variáveis foram chamadas Paciente, Trim, Rep, Entrev e Colesterol. A declaração DATALINES indica ao SAS que as observações são completadas linha por linha. Por exemplo, temos o conjunto de dados BLUP com algumas observações.

\section{- Dados no SAS}

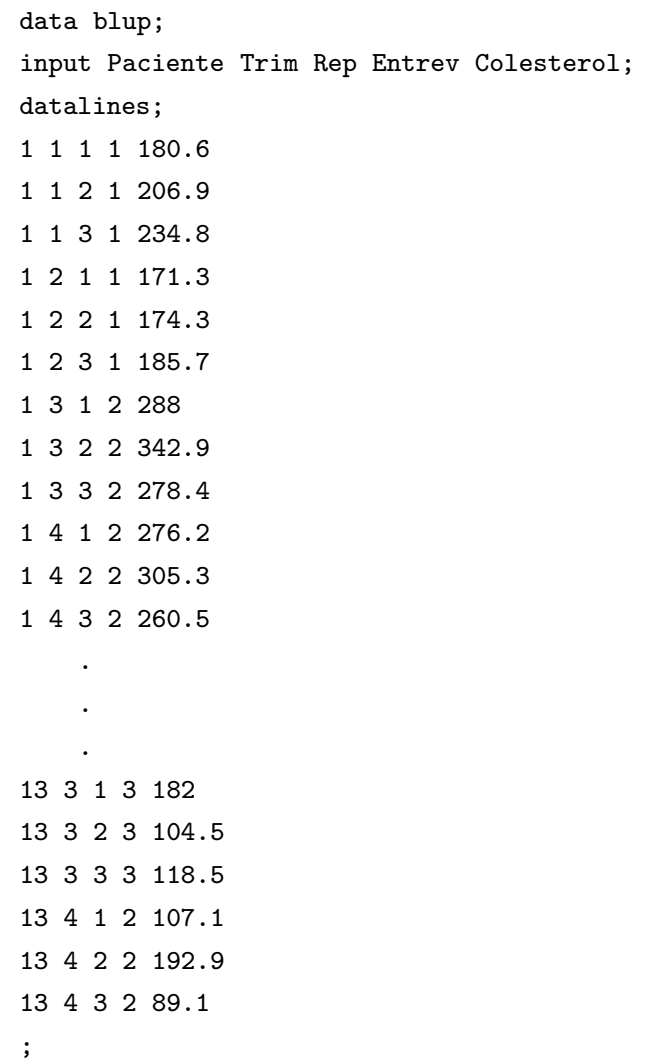




\section{- Resultados SAS}

The Mixed Procedure

Model Information

Data Set WORK. BLUP

Dependent Variable Colesterol

Covariance Structure Variance Components

Estimation Method REML

Residual Variance Method Profile

Fixed Effects SE Method Model-Based

Degrees of Freedom Method Containment

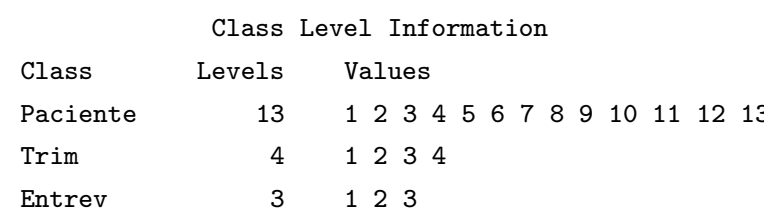

Dimensions

Covariance Parameters

Columns in $\mathrm{X}$

2

1

Columns in $\mathrm{Z}$

Subjects 1

Max Obs Per Subject 156

Number of Observations

Number of Observations Read 156

Number of Observations Used 156

Number of Observations Not Used 0

\begin{tabular}{rrrrr} 
& \multicolumn{2}{c}{ Iteration History } & \\
Iteration & Evaluations & -2 Res Log Like & Criterion \\
0 & 1 & 1746.21425159 & \\
1 & 1 & 1645.30973001 & 0.00000000
\end{tabular}

Covariance Parameter

Estimates

Cov Parm Estimate

Paciente 2788.25

Residual $\quad 1836.47$

Fit Statistics

-2 Res Log Likelihood 1645.3

AIC (smaller is better) 1649.3

AICC (smaller is better) 1649.4

BIC (smaller is better) 1650.4 


$\begin{array}{lrrrrr}\text { Label } & \text { Estimate } & \text { St.Error } & \text { DF } & \text { t Value } & \text { Pr }>|t| \\ \text { BLUP pac1 } & 240.99 & 12.0702 & 12 & 19.97 & <.0001 \\ \text { BLUP pac2 } & 260.99 & 12.0702 & 12 & 21.62 & <.0001 \\ \text { BLUP pac3 } & 157.94 & 12.0702 & 12 & 13.09 & <.0001 \\ \text { BLUP pac4 } & 231.98 & 12.0702 & 12 & 19.22 & <.0001 \\ \text { BLUP pac5 } & 203.16 & 12.0702 & 12 & 16.83 & <.0001 \\ \text { BLUP pac6 } & 277.29 & 12.0702 & 12 & 22.97 & <.0001 \\ \text { BLUP pac7 } & 265.92 & 12.0702 & 12 & 22.03 & <.0001 \\ \text { BLUP pac8 } & 199.56 & 12.0702 & 12 & 16.53 & <.0001 \\ \text { BLUP pac9 } & 299.10 & 12.0702 & 12 & 24.78 & <.0001 \\ \text { BLUP pac10 } & 236.81 & 12.0702 & 12 & 19.62 & <.0001 \\ \text { BLUP pac11 } & 145.90 & 12.0702 & 12 & 12.09 & <.0001 \\ \text { BLUP pac12 } & 215.58 & 12.0702 & 12 & 17.86 & <.0001 \\ \text { BLUP pac13 } & 133.58 & 12.0702 & 12 & 11.07 & <.0001\end{array}$

\section{- Resultados R}

Linear mixed-effects model fit by REML Data: BD1

Log-restricted-likelihood: -822.6549

Fixed: Colesterol 1

(Intercept)

220.6769

Random effects:

Formula: 1 | Paciente

(Intercept) Residual

StdDev: $\quad 52.8038642 .85405$

Number of Observations: 156

Number of Groups: 13

$>$ fit 1 \$coefficients\$fixed+fit1\$coefficients\$random\$Paciente

(Intercept)

$13 \quad 133.5805$

$11 \quad 145.9041$

$3 \quad 157.9433$

$12 \quad 215.5798$

$5 \quad 203.1614$

$8 \quad 199.5591$

$10 \quad 236.8143$

$2 \quad 260.9875$

$4 \quad 231.9796$

$6 \quad 277.2925$

1240.9853

$7 \quad 265.9169$

$9 \quad 299.0958$ 


\section{- Comentários sobre os resultados SAS e R}

- Nos dois programas, as estimativas dos componentes de variância são iguais e calculadas por meio do método de máxima verossimilhança restrita (REML), que é o método padrão (default). A estimativa da variância populacional dos valores latentes é $\hat{\gamma}^{2}=2788,25=(52,80386)^{2}$, sendo $94,8 \%$ da verdadeira variância $\gamma^{2}$. A estimativa da variância média dos erros de medida endógenos é $\hat{\bar{\sigma}}^{2}=1836,47=(42,85405)^{2}$, sendo $99,9 \%$ da verdadeira variância $\bar{\sigma}^{2}$. No SAS, os BLUP para os valores latentes correspondentes aos 13 indivíduos são dadas conjuntamente com seus desvios padrão; já no R somente temos o BLUP para cada indivíduo.

- Também geramos 1000 conjuntos de 156 observações (13 grupos de 12 observações cada). Cada grupo de 12 observações foi gerado a partir da distribuição normal com média $y_{s}$ e variância endógena correspondente $\sigma_{s}^{2}, s=$ $1,2, \ldots, 13$. Ajustamos o modelo misto usual e obtivemos que a média (dos 1000 conjuntos) da estimativa da variância populacional dos valores latentes é $\hat{\gamma}^{2}=2921,52$ e a média da estimativa da variância dos erros de medida endógenos é $\hat{\bar{\sigma}}^{2}=1822,89$ (Veja o código R no Apêndice K), sub-estimando em média os verdadeiros valores das variâncias. O desvío porcentual dessas estimativas relativamente às verdadeiras variâncias são - $-0,63 \%$ e - $0,74 \%$ respectivamente.

Também pode-se verificar que a estimativa da variância dos erros de medida endógenos é igual à média das variâncias endógenas, estimando essas variâncias individuais (Tabela 4.1) com as seguintes opções do PROC MIXED do SAS.

\section{- Código SAS:}

proc mixed;

class Paciente Trim Entrev;

model Colesterol=;

random Paciente;

repeated / group=Paciente;

\section{- Resultados SAS:}




$\begin{array}{llr}\text { Cov Parm } & \text { Group } & \begin{array}{r}\text { Estimate } \\ \text { Paciente }\end{array} \\ \text { Residual } & \text { Paciente } 1 & 3521.82 \\ \text { Residual } & \text { Paciente } 2 & 1249.01 \\ \text { Residual } & \text { Paciente } 3 & 1937.89 \\ \text { Residual } & \text { Paciente } 4 & 3062.85 \\ \text { Residual } & \text { Paciente } 5 & 1295.81 \\ \text { Residual } & \text { Paciente } 6 & 1014.78 \\ \text { Residual } & \text { Paciente } 7 & 2215.33 \\ \text { Residual } & \text { Paciente } 8 & 1808.72 \\ \text { Residual } & \text { Paciente } 9 & 5304.09 \\ \text { Residual } & \text { Paciente } 10 & 548.25 \\ \text { Residual } & \text { Paciente } 11 & 1103.19 \\ \text { Residual } & \text { Paciente } 12 & 638.11 \\ \text { Residual } & \text { Paciente } 13 & 208.04\end{array}$

Dado que estamos numa situação balanceada (os 13 indivíduos medidos 12 vezes cada), a média das estimativas das variâncias endógenas individuais é a estimativa da variância dos erros de medida endógenos, $\hat{\bar{\sigma}}^{2}=\frac{1}{13} \sum_{s=1}^{13} \hat{\sigma}_{s}^{2}$. Para situações desbalanceadas, deve se usar uma média ponderada pelo número de medições feitas em cada indivíduo.

\subsection{Modelos com erros de medida exógenos apenas}

Os preditores obtidos sob os modelos misto usual e MMPF são iguais na presença de erros de medida exógenos heterocedásticos. Podemos calcular as estimativas das variâncias exógenas e a variância populacional dos valores latentes sob o modelo misto usual com erros heterocedásticos usando os seguintes códigos escritos no PROC MIXED do SAS ou com a função lme da sub-rotina nlme do R.

\section{- Código SAS}

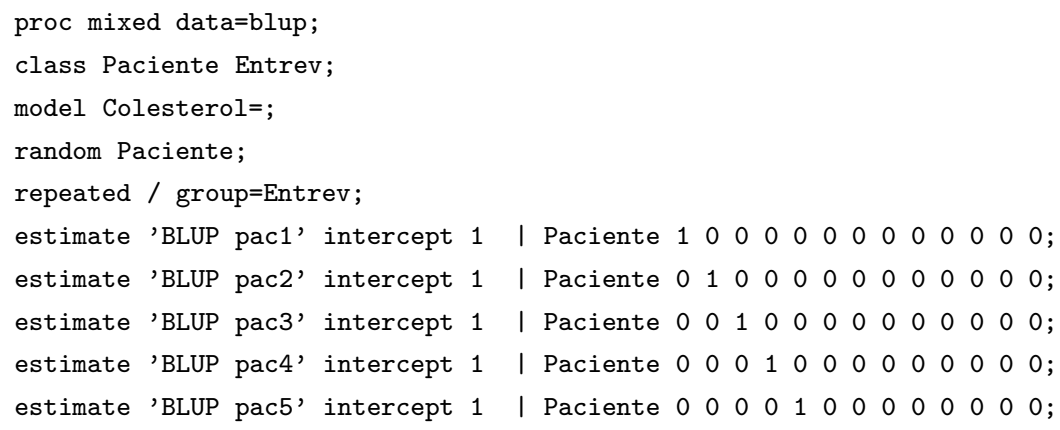




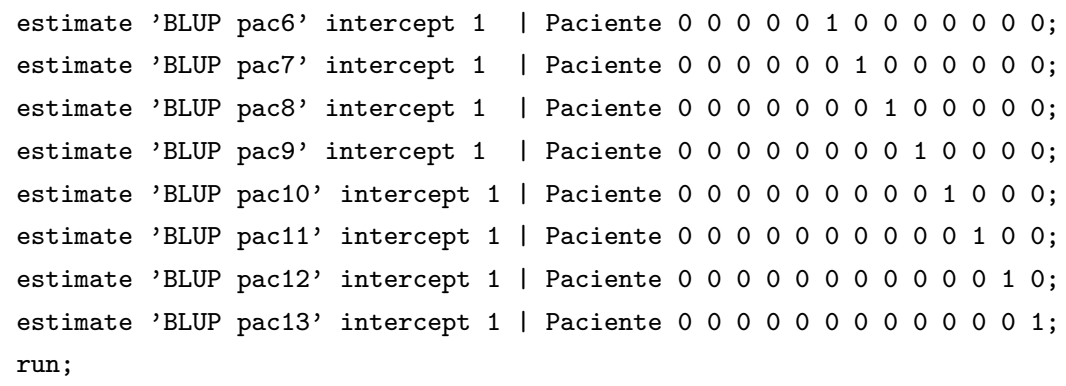

\section{- Código R}

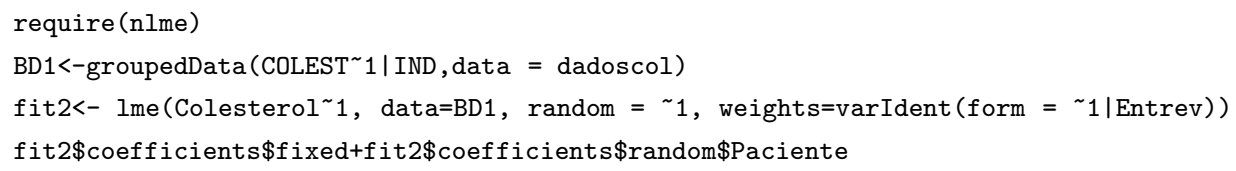

A partir dos valores latentes apresentados na Tabela 4.1 e das variâncias exógenas apresentadas na Tabela 1.4 geramos um conjunto de 156 observações. Cada grupo de 12 observações foi obtida como a soma do valor latente (da Tabela 4.1) e de um erro de medida exógeno obtido da seguinte forma: dividimos o intervalo [0,1] em 13 intervalos de igual tamanho e obtivemos os percentis correspondentes ao limite superior de cada intervalo da distribuição normal; transformamos esses dados tal que sua média seja zero e sua variância coincida com a variância exógena, $\tilde{\sigma}_{i}^{2}, i=1,2,3$, sendo estes nossos erros exógenos. Somamos a cada valor latente $y_{s}, s=1,2, \ldots, 13$, um conjunto dos erros exógenos gerados anteriormente considerando uma ordem particular, neste caso, por exemplo, admitimos que os indivíduos com rótulo $s=\{1,2,3, \ldots, 12,13\}$ foram avaliados pelos examinadores, na sequencia $\{C S, K L, K L, C S, K L, S U, C S, S U, C S, C S, S U, S U, K L\}$.

\section{- Resultados SAS:}

\begin{tabular}{lcc} 
& \multicolumn{2}{c}{ Covariance Parameter Estimates } \\
Cov Parm & Group & Estimate \\
Paciente & & 2797.15 \\
Residual & Entrev 1 & 1012.35 \\
Residual & Entrev 2 & 1623.10 \\
Residual & Entrev 3 & 2004.53 \\
\multicolumn{3}{c}{ Fit Statistics } \\
-2 Res Log Likelihood & 1610.9 \\
AIC (smaller is better) & 1618.9
\end{tabular}




$\begin{array}{ll}\text { AICC (smaller is better) } & 1619.2 \\ \text { BIC (smaller is better) } & 1621.2\end{array}$

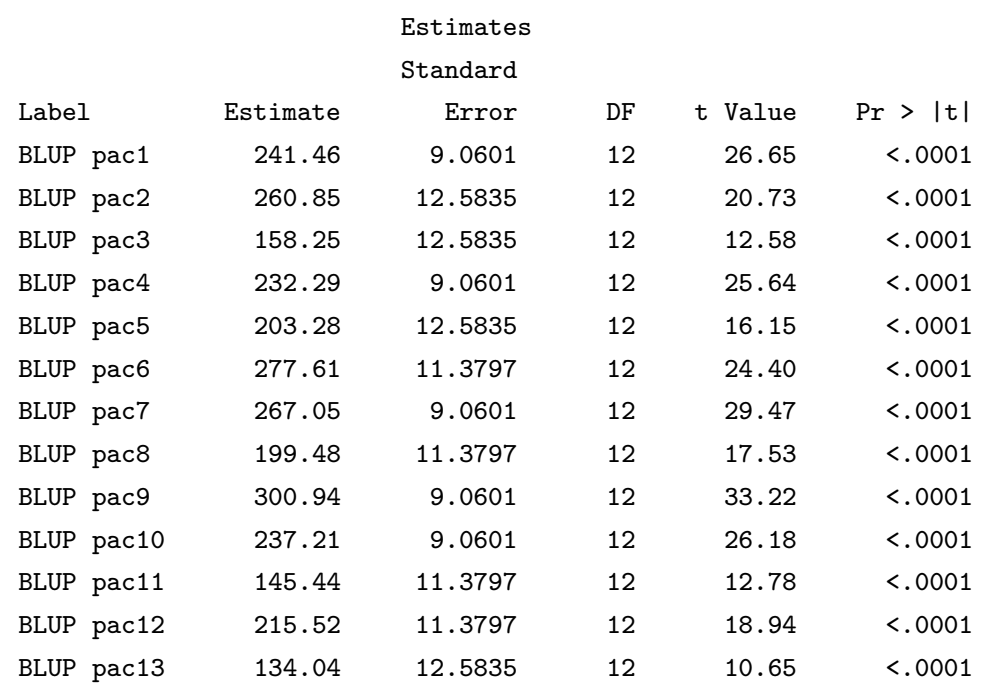

\section{- Resultados R:}

Linear mixed-effects model fit by REML

Data: BD2

Log-restricted-likelihood: -805.4476

Fixed: colesterol 1

(Intercept)

221.0324

Random effects:

Formula: 1 | Paciente

(Intercept) Residual

StdDev: $\quad 52.8880744 .77196$

Variance function:

Structure: Different standard deviations per stratum

Formula: 1 | Entrev

Parameter estimates:

3

$2 \quad 1$

$1.0000000 \quad 0.89984190 .7106595$

Number of Observations: 156

Number of Groups: 13

$>$ fit $2 \$$ coefficients $\$$ fixed+fit $2 \$$ coefficients $\$$ random $\$$ Paciente

(Intercept)

$13 \quad 134.0370$

$11 \quad 145.4387$

$3 \quad 158.2494$ 


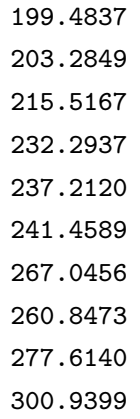

\section{- Comentários sobre os resultados SAS e R}

As estimativas dos componentes de variância foram calculadas com o método REML. A estimativa da variância populacional é $\hat{\gamma}^{2}=2797,15=(52,88807)^{2}$, sendo $95,1 \%$ da verdadeira variância dos valores latentes, $\gamma^{2}$. Os valores das estimativas das variâncias exógenas são fornecidas diretamente no SAS, já no R existe uma forma diferente de representação, ${ }^{1}$ elas são $\hat{\tilde{\sigma}}_{C S}^{2}=1012,4=(0,7106595 \times 44,77196)^{2}$, $\hat{\tilde{\sigma}}_{S U}^{2}=1623,1=(0,8998419 \times 44,77196)^{2}$ e $\hat{\tilde{\sigma}}_{K L}^{2}=2004,5=(1,0000 \times 44,77196)^{2}$, sendo 99,9\%, 99,9\% e 100,2\% respectivamente das verdadeiras variâncias exógenas.

\subsection{Modelos com presença simultânea de erros de medida endógenos e exógenos homocedásticos}

Podemos ajustar modelos em situações em que há de erros de medida endógenos e exógenos homocedásticos simultaneamente notando que o modelo misto usual para $k$ ésima medida da $i$-ésima unidade selecionada na amostra medida com a $j$-ésima condição de avaliação pode ser escrito como

$$
\begin{gathered}
Y_{i j k}=\mu+B_{i}+E_{i k}+\epsilon_{i j k}, \quad i=1, \ldots, n, \quad k=1, \ldots, n_{i}, \quad j=1,2, \ldots, p, \\
B_{i} \stackrel{i i d}{\sim} N\left(0, \gamma^{2}\right), \quad E_{i k} \stackrel{i i d}{\sim} N\left(0, \bar{\sigma}^{2}\right), \text { e } \epsilon_{i j k} \stackrel{i i d}{\sim} N\left(0, \tilde{\sigma}^{2}\right),
\end{gathered}
$$

em que $E_{i k}$ representa o erro de medida endógeno com variância homocedástica $\left(\bar{\sigma}^{2}\right)$ e $\epsilon_{i j k}$ representa o erro de medida exógeno com variância homocedástica $\left(\tilde{\sigma}^{2}\right)$.

\footnotetext{
${ }^{1}$ No processo de estimação das variâncias de cada condição de avaliação no R usa-se a expressão $\mathbb{V}\left(E_{i j}\right)=$ $\sigma^{2} \delta_{i}^{2} \operatorname{com} \delta_{1}=1$ e $\delta_{l}, l=2, \ldots, n$, representando a razão entre a $l$-ésima condição e a primeira condição selecionada.
} 
Nesta Seção usamos o conjunto de dados do Seasons Study, supondo que os erros de medida exógenos são homocedásticos. Podemos estimar as variâncias $\gamma^{2}, \bar{\sigma}^{2}$ e $\tilde{\sigma}^{2}$, e calcular os BLUP para os valores latentes correspondentes aos 13 indivíduos usando os seguintes códigos escritos no PROC MIXED do SAS ou com a função lme da sub-rotina nlme do R.

\section{- Código SAS}

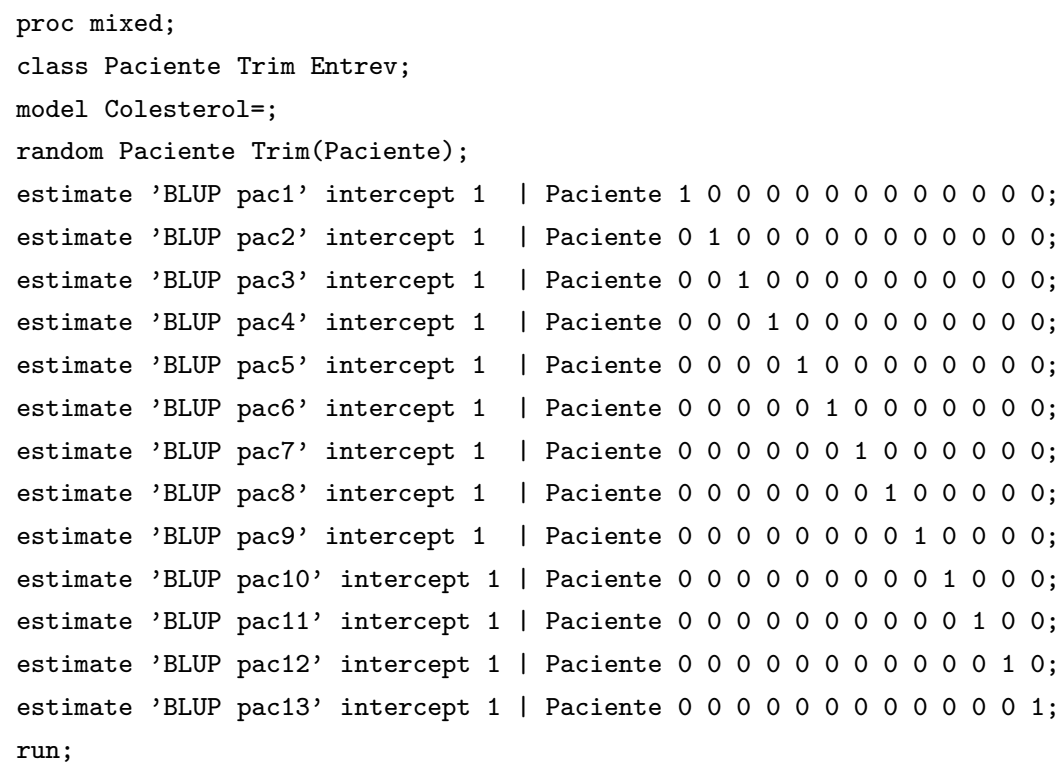

\section{- Código R}

require (nlme)

BLUP<- read.table("colesterol.txt", header $=T$ )

BLUP\$Paciente<-as.factor (BLUP\$Paciente)

BD2<-groupedData (Colesterol Entrev|Paciente $/$ Trim, data $=$ BLUP)

fit3<- lme(Colesterol 1 , data=BD2, random $=\sim 1$ )

fit3\$coefficients\$fixed+fit3\$coefficients\$random\$Paciente

\section{- Resultados SAS:}

The Mixed Procedure

Model Information

$\begin{array}{ll}\text { Data Set } & \text { WORK.BLUP } \\ \text { Dependent Variable } & \text { Colesterol } \\ \text { Covariance Structure } & \text { Variance Components }\end{array}$




\begin{tabular}{|c|c|c|c|c|}
\hline \multirow{2}{*}{\multicolumn{3}{|c|}{$\begin{array}{l}\text { Estimation Method } \\
\text { Residual Variance Method }\end{array}$}} & & REML \\
\hline & & & & Profile \\
\hline \multicolumn{3}{|c|}{ Fixed Effects SE Method } & & Model-Based \\
\hline \multirow[t]{2}{*}{ Degrees of } & Freedom $\mathrm{N}$ & lethod & & Containment \\
\hline & Class & Level & $\operatorname{Inf}$ & ormation \\
\hline Class & Levels & Valu & des & \\
\hline Paciente & 13 & 12 & 34 & $\begin{array}{lllllllll}5 & 6 & 7 & 8 & 9 & 10 & 11 & 12 & 13\end{array}$ \\
\hline Trim & 4 & 12 & 34 & \\
\hline Entrev & 3 & 12 & & \\
\hline
\end{tabular}

Dimensions

Covariance Parameters

3
1
65
1
156

Number of Observations

Number of Observations Read 156

Number of Observations Used 156

Number of Observations Not Used 0

Iteration History

$\begin{array}{rrrr}\text { Iteration } & \text { Evaluations } & -2 \text { Res Log Like } & \text { Criterion } \\ 0 & 1 & 1770.01717225 & \\ 1 & 1 & 1670.92679345 & 0.00000000\end{array}$

Covariance Parameter

$$
\text { Estimates }
$$

$\begin{array}{lr}\text { Cov Parm } & \text { Estimate } \\ \text { Paciente } & 2480.78 \\ \text { Trim(Paciente) } & 1317.72 \\ \text { Residual } & 1556.19\end{array}$

Fit Statistics

-2 Res Log Likelihood

1670.9

AIC (smaller is better)

1676.9

AICC (smaller is better)

1677.1

BIC (smaller is better)

1678.6

\begin{tabular}{lrrrrr}
\multicolumn{2}{c}{ Estimates } \\
Label & Estimate & Error & DF & t Value & Pr $>\mid$ t $\mid$ \\
BLUP pac1 & 238.73 & 19.8225 & 12 & 12.04 & $<.0001$ \\
BLUP pac2 & 256.57 & 19.8225 & 12 & 12.94 & $<.0001$
\end{tabular}




$\begin{array}{lrrrrr}\text { BLUP pac3 } & 164.83 & 19.8225 & 12 & 8.32 & <.0001 \\ \text { BLUP pac4 } & 230.77 & 19.8225 & 12 & 11.64 & <.0001 \\ \text { BLUP pac5 } & 205.10 & 19.8225 & 12 & 10.35 & <.0001 \\ \text { BLUP pac6 } & 271.04 & 19.8225 & 12 & 13.67 & <.0001 \\ \text { BLUP pac7 } & 260.98 & 19.8225 & 12 & 13.17 & <.0001 \\ \text { BLUP pac8 } & 201.91 & 19.8225 & 12 & 10.19 & <.0001 \\ \text { BLUP pac9 } & 290.44 & 19.8225 & 12 & 14.65 & <.0001 \\ \text { BLUP pac10 } & 235.04 & 19.8225 & 12 & 11.86 & <.0001 \\ \text { BLUP pac11 } & 154.11 & 19.8225 & 12 & 7.77 & <.0001 \\ \text { BLUP pac12 } & 216.10 & 19.8225 & 12 & 10.90 & <.0001 \\ \text { BLUP pac13 } & 143.18 & 19.8225 & 12 & 7.22 & <.0001\end{array}$

\section{- Resultados R:}

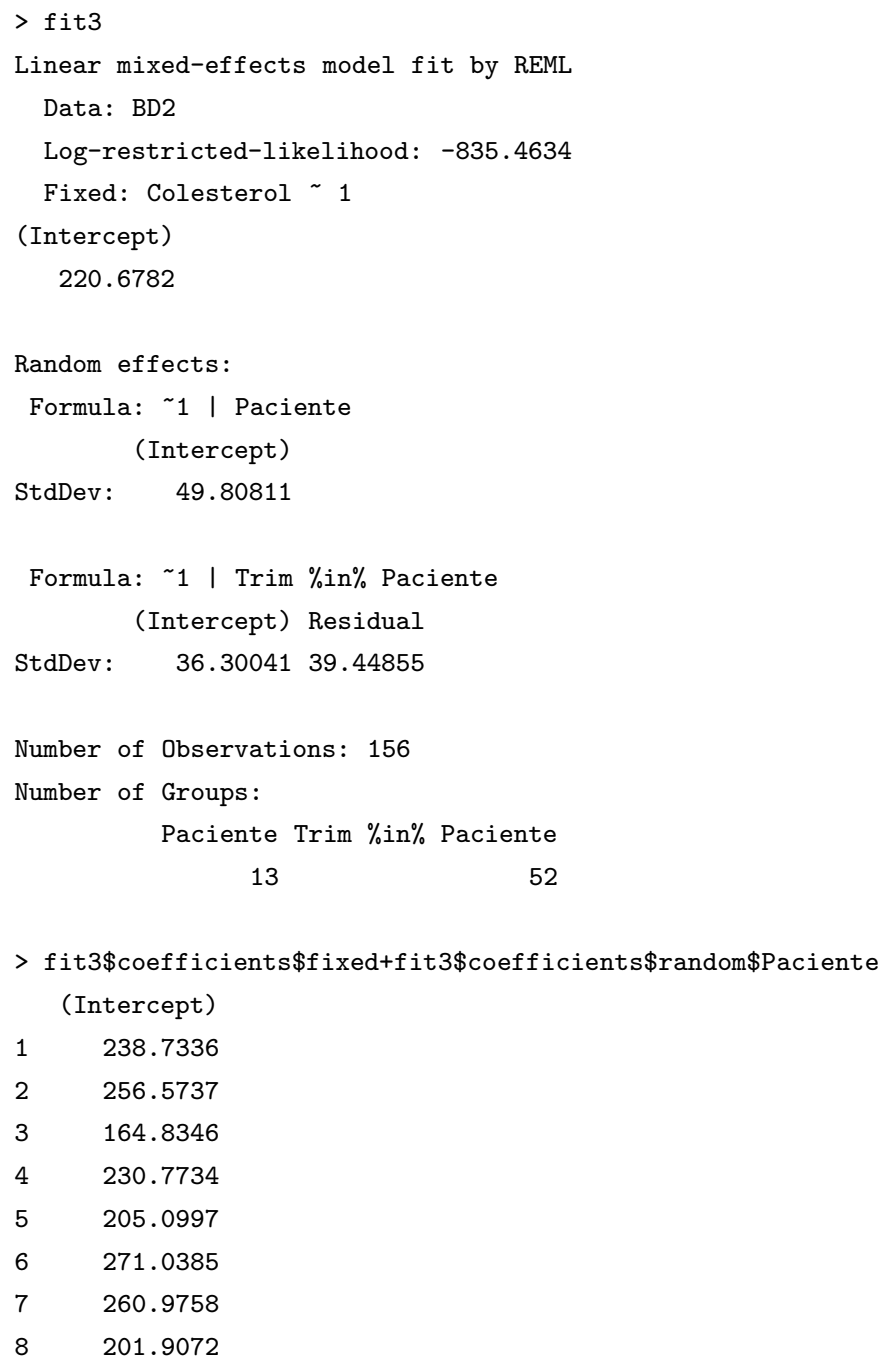




$\begin{array}{ll}9 & 290.4397 \\ 10 & 235.0418 \\ 11 & 154.1108 \\ 12 & 216.1047 \\ 13 & 143.1831\end{array}$

\section{- Comentários sobre os resultados SAS e R}

As estimativas dos componentes de variância foram calculadas com o método REML. A estimativa da variância populacional dos valores latentes é $\hat{\gamma}^{2}=(49,80811)^{2}=$ 2480,78, a estimativa da variância média dos erros de medida endógenos é $\hat{\bar{\sigma}}^{2}=$ $(36,30041)^{2}=1317,72$ e a estimativa da variância dos erros de medida exógenos é $\hat{\tilde{\sigma}}^{2}=(39,44855)^{2}=1556,19$.

\subsection{Modelos com presença simultânea de erros de medida endógenos e exógenos heterocedásticos: análise do exemplo Seasons Study}

Para ajustar modelos com presença simultânea de erros de medida exógenos heterocedásticos e endógenos homocedásticos ou heterocedásticos, podemos mudar no modelo

misto usual (4.5) a condição $\epsilon_{i j k} \stackrel{i i d}{\sim} N\left(0, \tilde{\sigma}^{2}\right)$ pela nova condição $\epsilon_{i j k} \stackrel{i i d}{\sim} N\left(0, \tilde{\sigma}_{j}^{2}\right)$ para $j=1,2, \ldots, p$, em que $p$ é o número de diferentes condições de avaliação.

Nas Seções (4.1) e (4.2), utilizamos o mesmo conjunto de dados do Seasons Study como base para a simulação num modelo com erro de medida endógeno ou exógeno apenas. Na Seção (4.3), ele foi utilizado supondo presença simultânea dos dois tipos de erros de medida, com erros de medida exógenos homocedásticos. Mas pode-se notar que estamos diante de uma situação em que há presença simultânea dos dois tipos de erros de medida e, além disso, os erros de medida exógenos são heterocedásticos.

Podemos estimar as variâncias $\gamma^{2}, \bar{\sigma}^{2}$ e $\tilde{\sigma}_{j}^{2}, \quad j=1,2,3$, e calcular os BLUP para os valores latentes correspondentes aos 13 indivíduos usando o seguinte código escrito no PROC MIXED do SAS ou com a função lme da sub-rotina nlme do R. 


\section{- Código SAS}

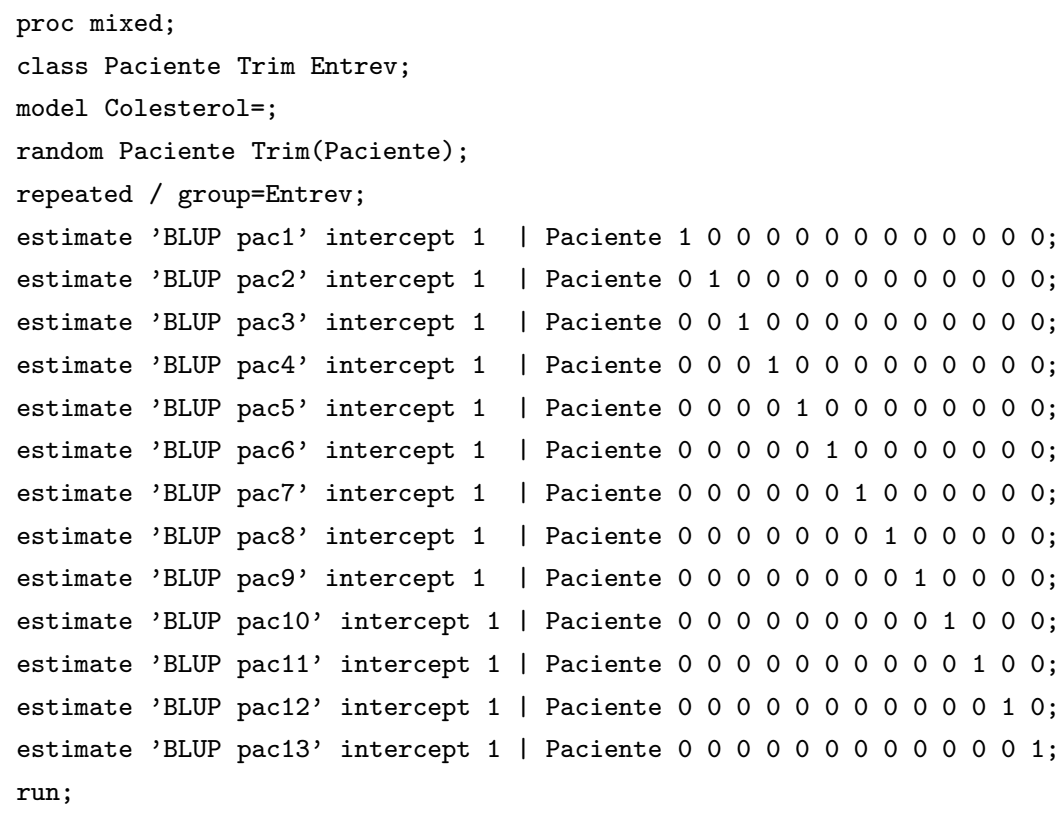

\section{- Código R}

BD4<-groupedData(Colesterol Entrev|Paciente $/$ Trim, data $=$ BLUP)

fit4<- lme(Colesterol $\sim 1$, data=BD4, random $=\sim 1$, weights=varIdent (form $=\sim 1 \mid$ Entrev))

fit 4 coefficients $\$$ fixed + fit 4 \$coefficients $\$$ random $\$$ Paciente

\section{- Resultados SAS:}

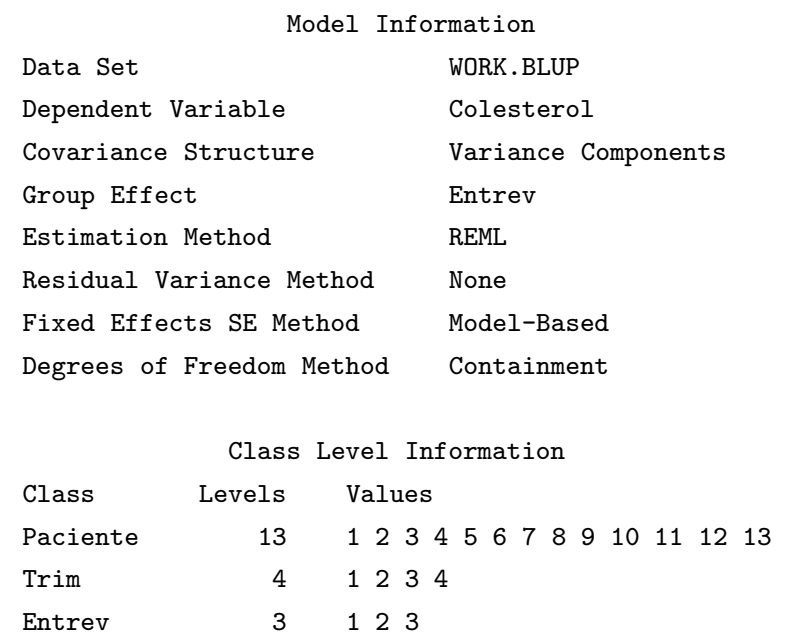




\section{Dimensions}

Covariance Parameters

Columns in $\mathrm{X}$

\section{5}

Columns in Z 65

Subjects

Max Obs Per Subject

Number of Observations

Number of Observations Read 156

Number of Observations Used 156

Number of Observations Not Used 0

$\begin{array}{rrrr}\text { Iteration } & \text { Evaluations } & -2 \text { Res Log Like } & \text { Criterion } \\ 0 & 1 & 1770.01717225 & \\ 1 & 2 & 1680.58242291 & 0.00000007 \\ 2 & 1 & 1669.51971858 & 0.00597302 \\ 3 & 1 & 1669.07828109 & 0.00186756 \\ 4 & 1 & 1667.53022074 & 0.00030372 \\ 5 & 1 & 1667.29540806 & 0.00001315 \\ 6 & 1 & 1667.28603627 & 0.00000003 \\ 7 & 1 & 1667.28601531 & 0.00000000\end{array}$

Covariance Parameter Estimates

$\begin{array}{llr}\text { Cov Parm } & \text { Group } & \text { Estimate } \\ \text { Paciente } & & 2455.09 \\ \text { Trim(Paciente) } & & 1312.78 \\ \text { Residual } & \text { Entrev 1 } & 1017.84 \\ \text { Residual } & \text { Entrev 2 } & 1592.82 \\ \text { Residual } & \text { Entrev 3 } & 2055.11\end{array}$

Fit Statistics

-2 Res Log Likelihood

1667.3

AIC (smaller is better) 1677.3

AICC (smaller is better) 1677.7

BIC (smaller is better) 1680.1

$\begin{array}{lrrrrr} & \text { Estimates } & & & \\ \text { Label } & \text { Estimate } & \text { Error } & \text { DF } & \text { t Value } & \text { Pr }>\mid \text { t } \mid \\ \text { BLUP pac1 } & 236.52 & 19.3672 & 12 & 12.21 & <.0001 \\ \text { BLUP pac2 } & 258.36 & 19.9226 & 12 & 12.97 & <.0001 \\ \text { BLUP pac3 } & 167.01 & 19.9226 & 12 & 8.38 & <.0001 \\ \text { BLUP pac4 } & 228.16 & 19.2911 & 12 & 11.83 & <.0001 \\ \text { BLUP pac5 } & 203.71 & 19.9226 & 12 & 10.22 & <.0001\end{array}$




$\begin{array}{llllrl}\text { BLUP pac6 } & 270.52 & 19.3672 & 12 & 13.97 & <.0001 \\ \text { BLUP pac7 } & 261.48 & 19.1427 & 12 & 13.66 & <.0001 \\ \text { BLUP pac8 } & 201.03 & 20.0064 & 12 & 10.05 & <.0001 \\ \text { BLUP pac9 } & 291.75 & 20.1760 & 12 & 14.46 & <.0001 \\ \text { BLUP pac10 } & 234.47 & 19.5209 & 12 & 12.01 & <.0001 \\ \text { BLUP pac11 } & 155.26 & 20.0064 & 12 & 7.76 & <.0001 \\ \text { BLUP pac12 } & 215.91 & 20.0064 & 12 & 10.79 & <.0001 \\ \text { BLUP pac13 } & 144.07 & 20.1760 & 12 & 7.14 & <.0001\end{array}$

\section{- Resultados R:}

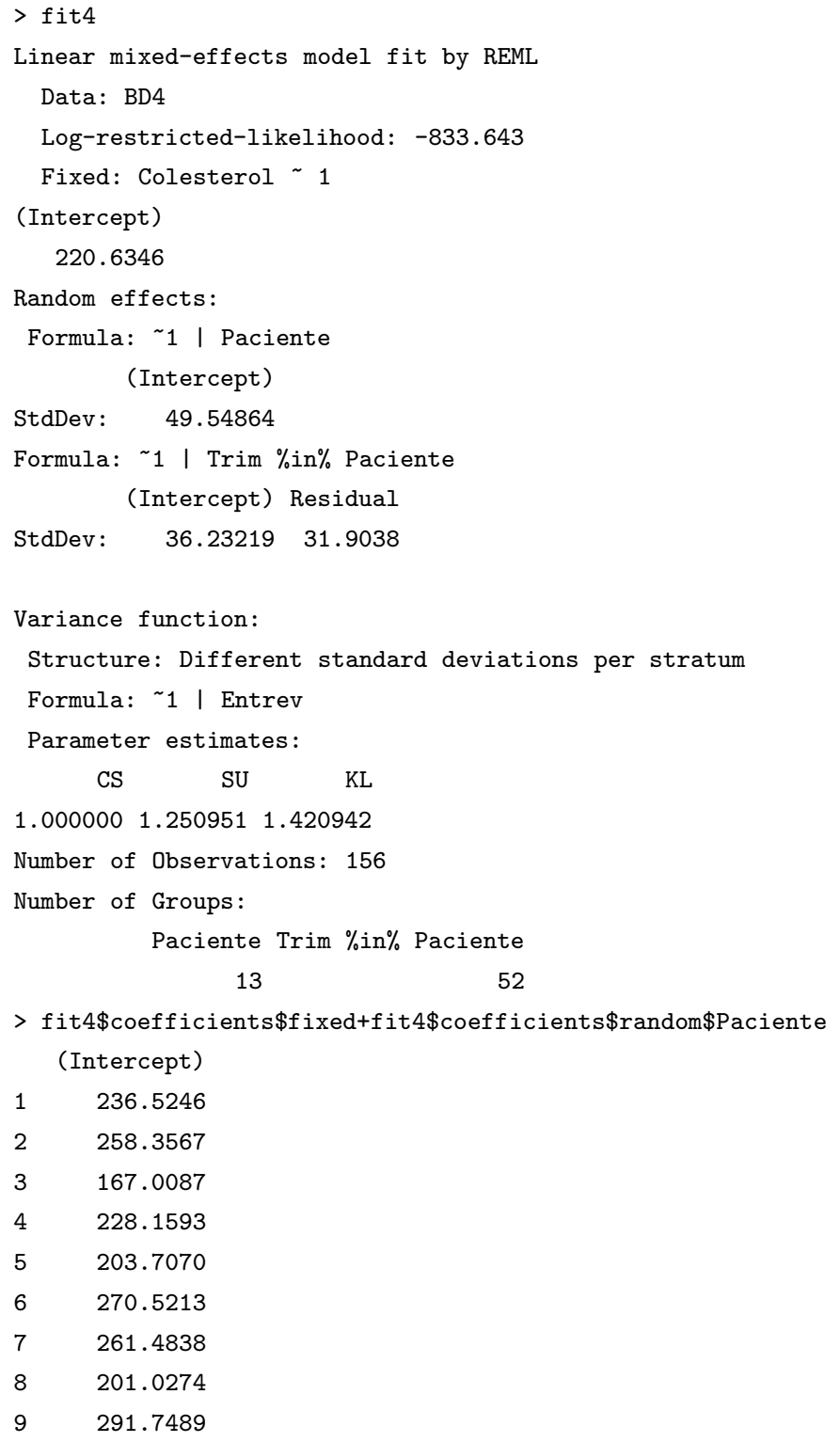




$\begin{array}{ll}10 & 234.4670 \\ 11 & 155.2619 \\ 12 & 215.9092 \\ 13 & 144.0745\end{array}$

- Descrição da saída:

No SAS e no R, temos que as estimativas dos componentes de variância são iguais e foram calculadas com o método REML. A estimativa da variância populacional dos valores latentes é $\hat{\gamma}^{2}=2455,09=(49,54864)^{2}$. A estimativa da média das variâncias endógenas é $\hat{\bar{\sigma}}=1312,78=(36,23219)^{2}$. As estimativas das variâncias exógenas, ou para cada condição de avaliação, são $\hat{\tilde{\sigma}}_{C S}^{2}=1017,84=(1 \times 31,9038)^{2}$, $\hat{\tilde{\sigma}}_{S U}^{2}=1592,82=(1,250951 \times 31,9038)^{2}$ e $\hat{\tilde{\sigma}}_{K L}^{2}=2055,11=(1,420942 \times 31,9038)^{2}$.

\subsection{Discussão}

O ajuste de modelos mistos com diferentes tipos de erros de medida pode ser realizado por intermédio dos pacotes computacionais disponíveis. Como o MMPF permite incorporar as duas fontes de erro de medida, endógenos e exógenos, ele pode ser utilizado para selecionar a estrutura de covariância adequada para ajustar o modelo no SAS ou no R.

Finalmente, o modelo misto para populações finitas com erros de medida endógenos e exógenos foi desenvolvido supondo uma medição em cada unidade amostral. É claro que dada a equivalência com os modelos mistos usuais, ele também pode ser usado para situações completamente desbalanceadas. 


\section{Capítulo 5}

\section{Conclusões}

Concentramos nosso estudo na estimação e predição ótima de diferentes quantidades populacionais com base em dados afetados por erros de medida endógenos e exógenos, obtidos a partir de amostras de populações finitas. Utilizamos o modelo misto para populações finitas com erros de medida exógenos e endógenos baseado no enfoque proposto por Stanek III et al. (2004) e Stanek III \& Singer (2004). A partir desse modelo calculamos o BLUP do valor latente da $i$-ésima unidade selecionada na amostra supondo conhecidas as variâncias endógenas. Quando as variâncias endógenas são heterocedásticas, o preditor obtido sob o MMPF é diferente daquele preditor obtido com o modelo misto usual, pois a constante de encolhimento depende da média das variâncias individuais. Isto indica que o BLUP obtido sob o MMPF não envolve a identificabilidade das unidades amostrais. Para tentar contornar esse problema consideramos um modelo mais geral com variáveis expandidas. Apesar de termos encontrado um preditor que leva em conta a variabilidade endógena do indivíduo, este não é aplicável pois requer o conhecimento a priori de todos os parâmetros individuais $y_{s}, s=1, \ldots, N$ e, consequentemente, ele muda para cada população, mostrando que não existe um estimador linear não enviesado e com variância uniformemente mínima para qualquer população com valores latentes $\boldsymbol{y}=\left(b_{1}, \ldots, b_{N}\right)^{\top}$. Assim, obtivemos uma generalização do resultado de Godambe (1955), que mostrou que para populações finitas não existe um estimador não enviesado com variância uniformemente mínima para o total populacional na classe de estimadores lineares, i.e., em que os coeficientes atribuídos às respostas observadas dependem tanto da unidade quanto da ordem de seleção na amostra selecionada, ou seja, exatamente a informação contida no 
vetor de variáveis expandidas.

Comparamos, via simulação, o preditor obtido sob o modelo misto usual (utilizado conforme a interpretação usual) com o preditor obtido sob o MMPF. Na maioria das situações consideradas, o estudo de simulação mostrou que o preditor obtido sob o modelo misto usual é enviesado, mas geralmente apresenta EQM menor (ou pouco maior em alguns casos) do que o EQM do preditor obtido sob o MMPF. Além disso, o desempenho deste preditor melhora à medida que aumentam o tamanho da população finita e a fração de amostragem. Tendo claras as limitações dos estudos de simulação e percebendo que podem ser necessárias pesquisas adicionais para obter conclusões mais definitivas quando existe erro de medida endógeno heterocedástico, os nossos resultados apontam na recomendação do preditor (2.10) obtido sob os modelos mistos usuais utilizado conforme a interpretação usual.

No Capítulo 3 mostramos que existem outras classes de estimadores que são não lineares e que geram EQM menores que os preditores da classe dos preditores lineares não enviesados. Pode-se pensar em outras classes de preditores que incluam o preditor (2.10), definir e avaliar a otimalidade nessas classes.

Finalmente, pode-se ampliar este estudo incorporando ao modelo estruturas diferentes à amostragem aleatória simples, para outros modelos com erros de medida e contemplar a estimação por intervalos e testes de hipótese. 


\section{Apêndice A}

\section{Detalhes sob cálculo dos BLUP}

A.1 Valor esperado e variância das variáveis aleatórias $\overleftrightarrow{Y}$

Dada a matriz $\boldsymbol{U}$, usando (2.22), a esperança condicional para as variáveis aleatórias expandidas é

$$
\mathbb{E}_{\xi_{2} \xi_{3} \mid \xi_{1}}(\stackrel{\leftrightarrow}{\boldsymbol{Y}})=\left[\boldsymbol{I}_{N} \otimes\left(\bigoplus_{s=1}^{N} y_{s}\right)\right] \operatorname{vec}\left(\boldsymbol{U}^{\top}\right)
$$

Utilizando (2.21), obtemos

$$
\mathbb{E}_{\xi_{1} \xi_{2} \xi_{3}}(\stackrel{\leftrightarrow}{\boldsymbol{Y}})=\left[\boldsymbol{I}_{N} \otimes\left(\bigoplus_{s=1}^{N} y_{s}\right)\right] \frac{1}{N}\left(\mathbf{1}_{N} \otimes \mathbf{1}_{N}\right)=\frac{1}{N}\left(\mathbf{1}_{N} \otimes \boldsymbol{y}\right)
$$

Para obter a variância, primeiro note que

$$
\mathbb{V}_{\xi_{1} \xi_{2} \xi_{3}}(\stackrel{\leftrightarrow}{\boldsymbol{Y}})=\mathbb{E}_{\xi_{1}}\left[\operatorname{var}_{\xi_{2} \xi_{3} \mid \xi_{1}}(\stackrel{\leftrightarrow}{\boldsymbol{Y}})\right]+\mathbb{V}_{\xi_{1}}\left[\mathbb{E}_{\xi_{2} \xi_{3} \mid \xi_{1}}(\stackrel{\leftrightarrow}{\boldsymbol{Y}})\right]
$$


Em seguida, observe que

$$
\begin{aligned}
\mathbb{V}_{\xi_{1}}\left[\mathbb{E}_{\xi_{2} \xi_{3} \mid \xi_{1}}(\stackrel{\boldsymbol{Y}}{)}]\right. & =\mathbb{V}_{\xi_{1}}\left\{\left[\boldsymbol{I}_{N} \otimes\left(\bigoplus_{s=1}^{N} y_{s}\right)\right] \operatorname{vec}\left(\boldsymbol{U}^{\top}\right)\right\} \\
& =\left[\boldsymbol{I}_{N} \otimes\left(\bigoplus_{s=1}^{N} y_{s}\right)\right] \frac{1}{N-1}\left(\boldsymbol{P}_{N} \otimes \boldsymbol{P}_{N}\right)\left[\boldsymbol{I}_{N} \otimes\left(\bigoplus_{s=1}^{N} y_{s}\right)\right] \\
& =\frac{1}{N-1}\left[\boldsymbol{I}_{N} \otimes\left(\bigoplus_{s=1}^{N} y_{s}\right)\right]\left[\boldsymbol{P}_{N} \otimes\left(\boldsymbol{P}_{N} \bigoplus_{s=1}^{N} y_{s}\right)\right] \\
& =\frac{1}{N-1}\left[\boldsymbol{P}_{N} \otimes\left(\bigoplus_{s=1}^{N} y_{s}\right) \boldsymbol{P}_{N}\left(\bigoplus_{s=1}^{N} y_{s}\right)\right] \\
& =\boldsymbol{P}_{N} \otimes \Delta,
\end{aligned}
$$

em que $\Delta=\frac{1}{N-1}\left(\bigoplus_{s=1}^{N} y_{s}\right) \boldsymbol{P}_{N}\left(\bigoplus_{s=1}^{N} y_{s}\right)$.

Além disso, temos

$$
\begin{aligned}
\mathbb{V}_{\xi_{2} \xi_{3} \mid \xi_{1}}(\stackrel{\leftrightarrow}{\boldsymbol{Y}})= & \mathbb{V}_{\xi_{2} \xi_{3} \mid \xi_{1}}\left[\operatorname{vec}\left(\overleftrightarrow{\boldsymbol{Y}}_{1} \overleftrightarrow{\boldsymbol{Y}}_{2} \ldots \overleftrightarrow{\boldsymbol{Y}}_{N}\right)\right] \\
= & \left(\begin{array}{ccc}
\mathbb{V}_{\xi_{2} \xi_{3} \mid \xi_{1}}\left(\overleftrightarrow{\boldsymbol{Y}}_{1}\right) & \ldots & \operatorname{cov}_{\xi_{2} \xi_{3} \mid \xi_{1}}\left(\overleftrightarrow{\boldsymbol{Y}}_{1}, \stackrel{\leftrightarrow}{\boldsymbol{Y}}_{N}^{\top}\right) \\
\operatorname{cov}_{\xi_{2} \xi_{3} \mid \xi_{1}}\left(\overleftrightarrow{\boldsymbol{Y}}_{2}, \overleftrightarrow{\boldsymbol{Y}}_{1}^{\top}\right) & \ldots & \operatorname{cov}_{\xi_{2} \xi_{3} \mid \xi_{1}}\left(\overleftrightarrow{\boldsymbol{Y}}_{2}, \overleftrightarrow{\boldsymbol{Y}}_{N}^{\top}\right) \\
\vdots & \ddots & \vdots \\
\operatorname{cov}_{\xi_{2} \xi_{3} \mid \xi_{1}}\left(\overleftrightarrow{\boldsymbol{Y}}_{N}, \overleftrightarrow{\boldsymbol{Y}}_{1}^{\top}\right) & \ldots & \mathbb{V}_{\xi_{2} \xi_{3} \mid \xi_{1}}\left(\overleftrightarrow{\boldsymbol{Y}}_{N}\right)
\end{array}\right),
\end{aligned}
$$

em que $\operatorname{cov}_{\xi_{2} \xi_{3} \mid \xi_{1}}\left(\overleftrightarrow{\boldsymbol{Y}}_{i}, \stackrel{\boldsymbol{Y}}{j}_{j}^{\top}\right)=\mathbf{0}_{N}, i \neq j, i=1, \ldots, N, j=1, \ldots, N$. Dada a independência entre os dois tipos de erros de medida podemos escrever

$$
\mathbb{V}_{\xi_{2} \xi_{3} \mid \xi_{1}}\left(\overleftrightarrow{\boldsymbol{Y}}_{i}\right)=\mathbb{V}_{\xi_{2} \mid \xi_{1}}\left\{\left(\bigoplus_{s=1}^{N} U_{i s}\right)\left(\begin{array}{c}
W_{1} \\
\vdots \\
W_{N}
\end{array}\right)\right\}+\mathbb{V}_{\xi_{3} \mid \xi_{1}}\left\{\tilde{W}_{i}\left(\begin{array}{c}
U_{i 1} \\
\vdots \\
U_{i N}
\end{array}\right)\right\}
$$

Então

$$
\mathbb{V}_{\xi_{2} \xi_{3} \mid \xi_{1}}\left(\overleftrightarrow{\boldsymbol{Y}}_{i}\right)=\bigoplus_{s=1}^{N}\left(U_{i s} \sigma_{s}^{2}\right)+\tilde{\sigma}_{i}^{2}\left(\begin{array}{cccc}
U_{i 1}^{2} & U_{i 1} U_{i 2} & \ldots & U_{i 1} U_{i N} \\
\vdots & \vdots & \ddots & \vdots \\
U_{i N} U_{i 1} & U_{i N} U_{i 2} & \ldots & U_{i N}^{2}
\end{array}\right)
$$


Dado que $\mathbb{E}_{\xi_{1}}\left(U_{i j}^{2}\right)=\frac{1}{N}$ e $\mathbb{E}_{\xi_{1}}\left(U_{i j} U_{i k}\right)=0, j \neq k, i=1, \ldots, N$, segue que

$$
\begin{aligned}
\mathbb{E}_{\xi_{1}}\left[\mathbb{V}_{\xi_{2} \xi_{3} \mid \xi_{1}}\left(\stackrel{\boldsymbol{Y}}{i}_{i}\right)\right] & =\mathbb{E}_{\xi_{1}}\left[\bigoplus_{s=1}^{N}\left(U_{i s} \sigma_{s}^{2}\right)+\tilde{\sigma}_{i}^{2}\left(\begin{array}{cccc}
U_{i 1}^{2} & U_{i 1} U_{i 2} & \ldots & U_{i 1} U_{i N} \\
\vdots & \vdots & \ddots & \vdots \\
U_{i N} U_{i 1} & U_{i N} U_{i 2} & \ldots & U_{i N}^{2}
\end{array}\right)\right] \\
& =\bigoplus_{s=1}^{N}\left(\frac{1}{N} \sigma_{s}^{2}\right)+\tilde{\sigma}_{i}^{2}\left(\bigoplus_{s=1}^{N} \frac{1}{N}\right) \\
& =\frac{1}{N} \bigoplus_{s=1}^{N}\left(\sigma_{s}^{2}+\tilde{\sigma}_{i}^{2}\right) .
\end{aligned}
$$

Então,

$$
\mathbb{E}_{\xi_{1}}\left[\mathbb{V}_{\xi_{2} \xi_{3} \mid \xi_{1}}(\stackrel{\leftrightarrow}{\boldsymbol{Y}})\right]=\boldsymbol{I}_{N} \otimes\left(\frac{1}{N} \bigoplus_{s=1}^{N} \sigma_{s}^{2}\right)+\left(\frac{1}{N} \bigoplus_{i=1}^{N} \tilde{\sigma}_{i}^{2}\right) \otimes \boldsymbol{I}_{N},
$$

e, finalmente, obtemos

$$
\mathbb{V}_{\xi_{1} \xi_{2} \xi_{3}}(\stackrel{\leftrightarrow}{\boldsymbol{Y}})=\boldsymbol{P}_{N} \otimes \Delta+\boldsymbol{I}_{N} \otimes\left(\frac{1}{N} \bigoplus_{s=1}^{N} \sigma_{s}^{2}\right)+\left(\frac{1}{N} \bigoplus_{i=1}^{N} \tilde{\sigma}_{i}^{2}\right) \otimes \boldsymbol{I}_{N}
$$

\section{A.2 Valor esperado e variância das variáveis aleatórias $\tilde{Y}$}

Dado que $\tilde{\boldsymbol{Y}}=\boldsymbol{L} \overleftrightarrow{\boldsymbol{Y}}$, com $\boldsymbol{L}=\boldsymbol{I}_{N} \otimes \mathbf{1}_{N}^{\top}$, temos

$$
\begin{aligned}
\mathbb{E}_{\xi_{1} \xi_{2} \xi_{3}}(\tilde{\boldsymbol{Y}}) & =\boldsymbol{L} \mathbb{E}_{\xi_{1} \xi_{2} \xi_{3}}(\overleftrightarrow{\boldsymbol{Y}}) \\
& =\left(\boldsymbol{I}_{N} \otimes \mathbf{1}_{N}^{\top}\right)\left(\frac{1}{N}\left(\mathbf{1}_{N} \otimes \boldsymbol{I}_{N}\right)\right) \boldsymbol{y} \\
& =\frac{1}{N}\left(\mathbf{1}_{N} \otimes \mathbf{1}_{N}^{\top}\right) \boldsymbol{y} \\
& =\mathbf{1}_{N} \mu
\end{aligned}
$$

e

$$
\begin{aligned}
\mathbb{V}_{\xi_{1} \xi_{2} \xi_{3}}(\tilde{\boldsymbol{Y}}) & =\boldsymbol{L} \mathbb{V}_{\xi_{1} \xi_{2} \xi_{3}}(\overleftrightarrow{\boldsymbol{Y}}) \boldsymbol{L}^{\top} \\
& =\boldsymbol{L}\left[\boldsymbol{P}_{N} \otimes \Delta+\boldsymbol{I}_{N} \otimes\left(\frac{1}{N} \bigoplus_{s=1}^{N} \sigma_{s}^{2}\right)+\left(\frac{1}{N} \bigoplus_{i=1}^{N} \tilde{\sigma}_{i}^{2}\right) \otimes \boldsymbol{I}_{N}\right] \boldsymbol{L}^{\top} \\
& =\gamma^{2} \boldsymbol{P}_{N}+\bar{\sigma}^{2} \boldsymbol{I}_{N}+\bigoplus_{i=1}^{N} \tilde{\sigma}_{i}^{2}
\end{aligned}
$$


em que $\bar{\sigma}^{2}=\frac{1}{N} \sum_{s=1}^{N} \sigma_{s}^{2}$ e $\quad \gamma^{2}=\mathbf{1}_{N}^{\top} \Delta \mathbf{1}_{N}=\frac{1}{N-1} \sum_{s=1}^{N}\left(y_{s}-\mu\right)^{2}$

\section{A.3 Obtenção do BLUP usando as variáveis $\overleftrightarrow{Y}$}

Um estimador linear não enviesado de variância mínima de $T=\boldsymbol{g}_{I}^{\prime} \ddot{\boldsymbol{Y}}_{I}+\boldsymbol{g}_{I I}^{\prime} \ddot{\boldsymbol{Y}}_{I I}$ baseado nas variáveis aleatórias $\overleftrightarrow{\boldsymbol{Y}}$ deve satisfazer as seguintes três condições:

i) Deve ser uma função linear das variáveis aleatórias observadas na amostra, i.e.,

$$
\hat{T}=\left(\boldsymbol{g}_{I}^{\prime}+\boldsymbol{a}^{\prime}\right) \stackrel{\leftrightarrow}{\boldsymbol{Y}}_{I}
$$

em que $\boldsymbol{g}_{I}$ é um vetor de constantes conhecidas.

ii) Deve ser não enviesado, i.e., $\mathbb{E}_{\xi_{1} \xi_{2} \xi_{3}}(\hat{T}-T)=0$,

$$
\begin{aligned}
\mathbb{E}_{\xi_{1} \xi_{2} \xi_{3}}(\hat{T}-T) & =\mathbb{E}_{\xi_{1} \xi_{2} \xi_{3}}\left(\left(\boldsymbol{g}_{I}^{\prime}+\boldsymbol{a}^{\prime}\right) \stackrel{\leftrightarrow}{\boldsymbol{Y}}_{I}-\boldsymbol{g}_{I}^{\prime} \boldsymbol{Y}_{I}-\boldsymbol{g}_{I I}^{\prime} \boldsymbol{Y}_{I I}\right) \\
& =\boldsymbol{g}_{I}^{\prime} \boldsymbol{X}_{I} \boldsymbol{y}+\boldsymbol{a}^{\prime} \boldsymbol{X}_{I} \boldsymbol{y}-\boldsymbol{g}_{I}^{\prime} \boldsymbol{X}_{I} \boldsymbol{y}-\boldsymbol{g}_{I I}^{\prime} \boldsymbol{X}_{I I} \boldsymbol{y} \\
& =\left(\boldsymbol{a}^{\prime} \boldsymbol{X}_{I}-\boldsymbol{g}_{I I}^{\prime} \boldsymbol{X}_{I I}\right) \boldsymbol{y}
\end{aligned}
$$

Para que esta expressão seja zero para todo valor de $\boldsymbol{y}$, deve-se satisfazer que ( $\boldsymbol{a}^{\prime} \boldsymbol{X}_{I}-$ $\left.\boldsymbol{g}_{I I}^{\prime} \boldsymbol{X}_{I I}\right)=0$, isto introduz uma restrição quando minimizamos o erro quadrático médio.

iii) Deve minimizar a $\mathbb{V}(\hat{T}-T)$.

Primeiro note que

$$
\begin{gathered}
\mathbb{V}_{\xi_{1} \xi_{2} \xi_{3}}\left(\begin{array}{c}
\overleftrightarrow{\boldsymbol{Y}}_{I} \\
\ddot{Y}_{I} \\
\ddot{Y}_{I I}
\end{array}\right)=\left(\begin{array}{ccc}
\overleftrightarrow{\boldsymbol{V}}_{I} & \ddot{\boldsymbol{V}}_{I} & \ddot{\boldsymbol{V}}_{I, I I} \\
\ddot{\boldsymbol{V}}_{I} & \ddot{\boldsymbol{V}}_{I} & \ddot{\boldsymbol{V}}_{I, I I} \\
\ddot{\boldsymbol{V}}_{I I, I} & \ddot{\boldsymbol{V}}_{I I, I} & \ddot{\boldsymbol{V}}_{I I}
\end{array}\right), \\
\operatorname{em~que}\left(\begin{array}{cc}
\ddot{\boldsymbol{V}}_{I} & \ddot{\boldsymbol{V}}_{I, I I} \\
\ddot{\boldsymbol{V}}_{I I, I} & \ddot{\boldsymbol{V}}_{I I}
\end{array}\right)=\mathbb{V}_{\xi_{1}}\left(\begin{array}{c}
\ddot{Y}_{I} \\
\ddot{Y}_{I I}
\end{array}\right) \mathrm{e} \overleftrightarrow{\boldsymbol{V}}_{I}=\mathbb{V}_{\xi_{1} \xi_{3} \xi_{3}}\left(\overleftrightarrow{\boldsymbol{Y}}_{I}\right) .
\end{gathered}
$$


Assim,

$$
\begin{aligned}
\mathbb{V}_{\xi_{1} \xi_{2} \xi_{3}}(\hat{T}-T) & =\left(\left(\boldsymbol{g}_{I}^{\prime}+\boldsymbol{a}^{\prime}\right)-\boldsymbol{g}_{I}^{\prime}-\boldsymbol{g}_{I I}^{\prime}\right)\left(\begin{array}{ccc}
\overleftrightarrow{\boldsymbol{V}}_{I} & \ddot{V}_{I} & \ddot{V}_{I, I I} \\
\ddot{V}_{I} & \ddot{V}_{I} & \ddot{V}_{I, I I} \\
\ddot{V}_{I I, I} & \ddot{V}_{I I, I} & \ddot{V}_{I I}
\end{array}\right)\left(\begin{array}{c}
\left(\boldsymbol{g}_{I}+\boldsymbol{a}\right) \\
-\boldsymbol{g}_{I} \\
-\boldsymbol{g}_{I I}
\end{array}\right) \\
& =\left(\boldsymbol{a}^{\prime}-\boldsymbol{g}_{I I}^{\prime}\right)\left(\begin{array}{cc}
\ddot{V}_{I} & \ddot{V}_{I, I I} \\
\ddot{V}_{I I, I} & \ddot{V}_{I I}
\end{array}\right)\left(\begin{array}{c}
\boldsymbol{a} \\
-\boldsymbol{g}_{I I}
\end{array}\right)+\left(\boldsymbol{g}_{I}^{\prime}+\boldsymbol{a}^{\prime}\right) \boldsymbol{V}_{I}^{*}\left(\boldsymbol{g}_{I}+\boldsymbol{a}\right),
\end{aligned}
$$

em que $\boldsymbol{V}_{I}^{*}=\stackrel{\boldsymbol{V}}{I}_{I}-\ddot{\boldsymbol{V}}_{I}$. Expandindo esta expressão, temos

$$
\mathbb{V}_{\xi_{1} \xi_{2} \xi_{3}}(\hat{T}-T)=\boldsymbol{a}^{\prime} \stackrel{\leftrightarrow}{\boldsymbol{V}}_{I} \boldsymbol{a}+2\left(\boldsymbol{g}_{I}^{\prime} \boldsymbol{V}_{I}^{*}-\boldsymbol{g}_{I I}^{\prime} \ddot{\boldsymbol{V}}_{I I, I}\right) \boldsymbol{a}+\boldsymbol{g}_{I I}^{\prime} \ddot{\boldsymbol{V}}_{I I} \boldsymbol{g}_{I I}+\boldsymbol{g}_{I}^{\prime} \boldsymbol{V}_{I}^{*} \boldsymbol{g}_{I}
$$

Incluindo a restrição de não enviesamento via multiplicadores de Lagrange, nosso objetivo é encontrar o valor de $\boldsymbol{a}$ que minimize

$f(\boldsymbol{a}, \lambda)=\boldsymbol{a}^{\prime} \stackrel{\leftrightarrow}{\boldsymbol{V}}_{I} \boldsymbol{a}+2\left(\boldsymbol{g}_{I}^{\prime} \boldsymbol{V}_{I}^{*}-\boldsymbol{g}_{I I}^{\prime} \ddot{\boldsymbol{V}}_{I I, I}\right) \boldsymbol{a}+\boldsymbol{g}_{I I}^{\prime} \ddot{\boldsymbol{V}}_{I I} \boldsymbol{g}_{I I}+\boldsymbol{g}_{I}^{\prime} \boldsymbol{V}_{I}^{*} \boldsymbol{g}_{I}+2 \lambda\left(\boldsymbol{a}^{\prime} \boldsymbol{X}_{I}-\boldsymbol{g}_{I I}^{\prime} \boldsymbol{X}_{I I}\right)$

Derivando com relação a $\boldsymbol{a}$ e $\lambda$, temos

$$
\begin{aligned}
& \frac{\partial f(\boldsymbol{a}, \lambda)}{\partial \boldsymbol{a}}=2 \overleftrightarrow{\boldsymbol{V}}_{I} \boldsymbol{a}+2\left(\boldsymbol{V}_{I}^{*} \boldsymbol{g}_{I}-\ddot{\boldsymbol{V}}_{I, I I} \boldsymbol{g}_{I I}\right)+2 \lambda \boldsymbol{X}_{I} \\
& \frac{\partial f(\boldsymbol{a}, \lambda)}{\partial \lambda}=2\left(\boldsymbol{X}_{I}^{\prime} \boldsymbol{a}-\boldsymbol{X}_{I I}^{\prime} \boldsymbol{g}_{I I}\right) .
\end{aligned}
$$

Igualando a zero simultaneamente as duas equações, temos

$$
\frac{1}{2}\left(\begin{array}{c}
\frac{\partial f(\hat{\boldsymbol{a}}, \hat{\lambda})}{\partial \boldsymbol{a}, \hat{\lambda}} \\
\frac{\partial f(\hat{\boldsymbol{a}}, \hat{\lambda})}{\partial \lambda}
\end{array}\right)=\left(\begin{array}{cc}
\overleftrightarrow{\boldsymbol{V}}_{I} & \boldsymbol{X}_{I} \\
\boldsymbol{X}_{I}^{\prime} & \mathbf{0}
\end{array}\right)\left(\begin{array}{c}
\hat{\boldsymbol{a}} \\
\hat{\lambda}
\end{array}\right)-\left(\begin{array}{c}
-\boldsymbol{V}_{I}^{*} \boldsymbol{g}_{I}+\ddot{\boldsymbol{V}}_{I, I I} \boldsymbol{g}_{I I} \\
\boldsymbol{X}_{I I}^{\prime} \boldsymbol{g}_{I I}
\end{array}\right)=\left(\begin{array}{c}
\mathbf{0}_{n} \\
\mathbf{0}
\end{array}\right),
$$

ou, de forma equivalente,

$$
\left(\begin{array}{cc}
\overleftrightarrow{\boldsymbol{V}}_{I} & \boldsymbol{X}_{I} \\
\boldsymbol{X}_{I}^{\prime} & \mathbf{0}
\end{array}\right)\left(\begin{array}{c}
\hat{\boldsymbol{a}} \\
\hat{\lambda}
\end{array}\right)=\left(\begin{array}{c}
-\boldsymbol{V}_{I}^{*} \boldsymbol{g}_{I}+\ddot{\boldsymbol{V}}_{I, I I} \boldsymbol{g}_{I I} \\
\boldsymbol{X}_{I I}^{\prime} \boldsymbol{g}_{I I}
\end{array}\right)
$$

Para resolver este sistema de equações, usamos o seguinte resultado de matrizes inversas particionadas:

$\mathrm{Se}$

$$
\boldsymbol{X}=\left(\begin{array}{ll}
\boldsymbol{A} & \boldsymbol{B} \\
\boldsymbol{C} & \boldsymbol{D}
\end{array}\right)
$$


então

$$
\boldsymbol{X}^{-1}=\left(\begin{array}{cc}
\boldsymbol{A}^{-1}+\boldsymbol{A}^{-1} \boldsymbol{B} \boldsymbol{Q}^{-1} \boldsymbol{C} \boldsymbol{A}^{-1} & -\boldsymbol{A}^{-1} \boldsymbol{B} \boldsymbol{Q}^{-1} \\
-\boldsymbol{Q}^{-1} \boldsymbol{C} \boldsymbol{A}^{-1} & \boldsymbol{Q}^{-1}
\end{array}\right),
$$

em que a matriz $\boldsymbol{Q}=\boldsymbol{D}-\boldsymbol{C} \boldsymbol{A}^{-1} \boldsymbol{B}$. Em consequencia,

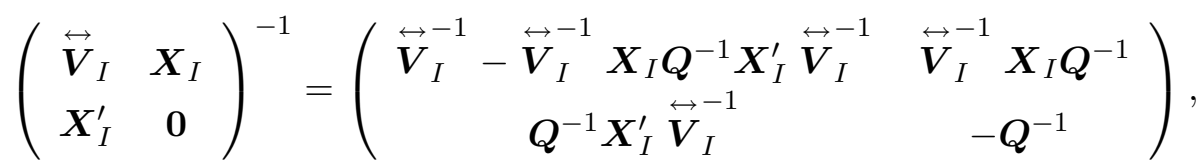

em que $\boldsymbol{Q}=\left(\boldsymbol{X}_{I}^{\prime} \overleftrightarrow{\boldsymbol{V}}_{I}^{-1} \boldsymbol{X}_{I}\right)$

Assim,

$$
\begin{aligned}
\hat{\boldsymbol{a}} & =\left[\begin{array}{ll}
\stackrel{\boldsymbol{V}}{I}_{I}^{-1}-\overleftrightarrow{\boldsymbol{V}}_{I}^{-1} \boldsymbol{X}_{I} \boldsymbol{Q}^{-1} \boldsymbol{X}_{I}^{\prime} \stackrel{\leftrightarrow}{\boldsymbol{V}}_{I}^{-1}
\end{array}\right]\left(-\boldsymbol{V}_{I}^{*} \boldsymbol{g}_{I}+\ddot{\boldsymbol{V}}_{I, I I} \boldsymbol{g}_{I I}\right) \\
& +\stackrel{\leftrightarrow}{\boldsymbol{V}}_{I}^{-1} \boldsymbol{X}_{I} \boldsymbol{Q}^{-1} \boldsymbol{X}_{I I}^{\prime} \boldsymbol{g}_{I I}
\end{aligned}
$$

e o melhor preditor linear não enviesado obtem-se substituindo $\boldsymbol{a}$ por $\hat{\boldsymbol{a}}$. Então,

$$
\hat{T}=\boldsymbol{g}_{I}^{\prime}\left[\boldsymbol{X}_{I} \hat{\boldsymbol{\alpha}}+\ddot{\boldsymbol{V}}_{I} \stackrel{\leftrightarrow}{\boldsymbol{V}}_{I}^{-1}\left(\overleftrightarrow{\boldsymbol{Y}}_{I}-\boldsymbol{X}_{I} \hat{\boldsymbol{\alpha}}\right)\right]+\boldsymbol{g}_{I I}^{\prime}\left[\boldsymbol{X}_{I I} \hat{\boldsymbol{\alpha}}+\ddot{\boldsymbol{V}}_{I I, I} \stackrel{\leftrightarrow}{\boldsymbol{V}}_{I}^{-1}\left(\overleftrightarrow{\boldsymbol{Y}}_{I}-\boldsymbol{X}_{I} \hat{\boldsymbol{\alpha}}\right)\right]
$$

em que $\hat{\boldsymbol{\alpha}}=\left(\boldsymbol{X}_{I}^{\prime} \stackrel{\leftrightarrow}{\boldsymbol{V}}_{I}^{-1} \boldsymbol{X}_{I}\right)^{-1} \boldsymbol{X}_{I}^{\prime} \stackrel{\leftrightarrow}{\boldsymbol{V}}_{I}^{-1} \overleftrightarrow{\boldsymbol{Y}}_{I}$

\section{A.4 Obtenção da variância de $\hat{P}$ usando as variáveis $\tilde{\boldsymbol{Y}}$}

De forma geral,

$$
\mathbb{V}_{\xi_{1} \xi_{2} \xi_{3}}(\hat{P}-P)=\hat{\boldsymbol{a}}^{\prime} \tilde{\boldsymbol{V}}_{I} \hat{\boldsymbol{a}}+2 \hat{\boldsymbol{a}}^{\prime}\left(\boldsymbol{V}_{I}^{*} \boldsymbol{g}_{I}-\boldsymbol{V}_{I I, I} \boldsymbol{g}_{I I}\right)+\boldsymbol{g}_{I}^{\prime} \boldsymbol{V}_{I}^{*} \boldsymbol{g}_{I}+\boldsymbol{g}_{I I}^{\prime} \boldsymbol{V}_{I I} \boldsymbol{g}_{I I}
$$

Usando as variáveis aleatórias $\tilde{\boldsymbol{Y}}$ temos

$$
\begin{aligned}
\boldsymbol{X}_{I} & =\mathbf{1}_{n} \\
\boldsymbol{X}_{I I} & =\mathbf{1}_{N-n}, \\
\tilde{\boldsymbol{V}}_{I} & =\gamma^{2}\left[\boldsymbol{I}_{n}-\frac{1}{N} \boldsymbol{J}_{n}\right]+\bar{\sigma}^{2} \boldsymbol{I}_{n}+\bigoplus_{i=1}^{n} \tilde{\sigma}_{i}^{2}
\end{aligned}
$$




$$
\begin{aligned}
\boldsymbol{V}_{I} & =\gamma^{2}\left[\boldsymbol{I}_{n}-\frac{1}{N} \boldsymbol{J}_{n}\right], \\
\boldsymbol{V}_{I I, I} & =-\frac{\gamma^{2}}{N} \boldsymbol{J}_{(N-n) \times n}, \\
\boldsymbol{V}_{I}^{*} & =\bigoplus_{i=1}^{n}\left(\bar{\sigma}^{2}+\tilde{\sigma}_{i}^{2}\right), \\
\tilde{\boldsymbol{V}}_{I}^{-1} & =\bigoplus_{i=1}^{n}\left(\gamma^{2}+\bar{\sigma}^{2}+\tilde{\sigma}_{i}^{2}\right)^{-1}+\frac{\gamma^{2}}{N-\gamma^{2} L} \mathbf{m m}^{\prime},
\end{aligned}
$$

em que $L=\sum_{i=1}^{n}\left(\gamma^{2}+\bar{\sigma}^{2}+\tilde{\sigma}_{i}^{2}\right)^{-1}$ e $\boldsymbol{m}$ é um vetor $n$ dimensional com $i$-ésima componente igual a $\left(\gamma^{2}+\bar{\sigma}^{2}+\tilde{\sigma}_{i}^{2}\right)^{-1}$.

Quando $i \leq n, \boldsymbol{g}_{I I}=\mathbf{0}$ e $\hat{\boldsymbol{a}}^{\prime}=\left(0 \cdots \frac{-\left(\bar{\sigma}^{2}+\tilde{\sigma}_{i}^{2}\right)}{\left(\gamma^{2}+\bar{\sigma}^{2}+\tilde{\sigma}_{i}^{2}\right)} \cdots 0\right)^{\prime}+\frac{\left(\bar{\sigma}^{2}+\tilde{\sigma}_{i}^{2}\right)}{L\left(\gamma^{2}+\bar{\sigma}^{2}+\tilde{\sigma}_{i}^{2}\right)} \boldsymbol{m}^{\prime}$. Também,

$$
\begin{aligned}
\hat{\boldsymbol{a}}^{\prime} \tilde{\boldsymbol{V}}_{I} \hat{\boldsymbol{a}} & =\frac{\left(\bar{\sigma}^{2}+\tilde{\sigma}_{i}^{2}\right)^{2}}{\left(\gamma^{2}+\bar{\sigma}^{2}+\tilde{\sigma}_{i}^{2}\right)}-\frac{\left(\bar{\sigma}^{2}+\tilde{\sigma}_{i}^{2}\right)^{2}}{L\left(\gamma^{2}+\bar{\sigma}^{2}+\tilde{\sigma}_{i}^{2}\right)^{2}} \\
\boldsymbol{g}_{I}^{\prime} \boldsymbol{V}_{I}^{*} \boldsymbol{g}_{I} & =\left(\bar{\sigma}^{2}+\tilde{\sigma}_{i}^{2}\right) \\
2 \hat{\boldsymbol{a}}^{\prime} \boldsymbol{V}_{I}^{*} \boldsymbol{g}_{I} & =\frac{-2\left(\bar{\sigma}^{2}+\tilde{\sigma}_{i}^{2}\right)^{2}}{\left(\gamma^{2}+\bar{\sigma}^{2}+\tilde{\sigma}_{i}^{2}\right)}+\frac{2\left(\bar{\sigma}^{2}+\tilde{\sigma}_{i}^{2}\right)^{2}}{L\left(\gamma^{2}+\bar{\sigma}^{2}+\tilde{\sigma}_{i}^{2}\right)^{2}}
\end{aligned}
$$

e

$$
\mathbb{V}_{\xi_{1} \xi_{2} \xi_{3}}\left(\hat{P}_{i}-P_{i}\right)=\frac{\left(\bar{\sigma}^{2}+\tilde{\sigma}_{i}^{2}\right)^{2}}{L\left(\gamma^{2}+\bar{\sigma}^{2}+\tilde{\sigma}_{i}^{2}\right)^{2}}-\frac{\left(\bar{\sigma}^{2}+\tilde{\sigma}_{i}^{2}\right)^{2}}{\left(\gamma^{2}+\bar{\sigma}^{2}+\tilde{\sigma}_{i}^{2}\right)}+\left(\bar{\sigma}^{2}+\tilde{\sigma}_{i}^{2}\right), \quad i \leq n .
$$

Quando $i>n, \boldsymbol{g}_{I}=\mathbf{0}$ e $\hat{\boldsymbol{a}}^{\prime}=\frac{1}{L} \boldsymbol{m}^{\prime}$. Também

$$
\begin{aligned}
\hat{\boldsymbol{a}}^{\prime} \tilde{\boldsymbol{V}}_{I} \hat{\boldsymbol{a}} & =\frac{1}{L}-\frac{\gamma^{2}}{N}, \\
\boldsymbol{g}_{I I}^{\prime} \boldsymbol{V}_{I I} \boldsymbol{g}_{I I} & =\gamma^{2}-\frac{\gamma^{2}}{N}, \\
-2 \hat{\boldsymbol{a}}^{\prime} \boldsymbol{V}_{I I, I} \boldsymbol{g}_{I I} & =2 \frac{\gamma^{2}}{N}
\end{aligned}
$$

e

$$
\mathbb{V}_{\xi_{1} \xi_{2} \xi_{3}}\left(\hat{P}_{i}-P_{i}\right)=\frac{1}{L}+\gamma^{2}, \quad i>n
$$




\section{Apêndice B}

\section{Dados do Nível de Colesterol}

data blup;

input Paciente Trim Rep Entrev Colesterol;

datalines;

$\begin{array}{lllll}1 & 1 & 1 & 1 & 180.6\end{array}$

$\begin{array}{lllll}1 & 1 & 2 & 1 & 206.9\end{array}$

$\begin{array}{lllll}1 & 1 & 3 & 1 & 234.8\end{array}$

$\begin{array}{lllll}1 & 2 & 1 & 1 & 171.3\end{array}$

$\begin{array}{lllll}1 & 2 & 2 & 1 & 174.3\end{array}$

$\begin{array}{lllll}1 & 2 & 3 & 1 & 185.7\end{array}$

$\begin{array}{lllll}1 & 3 & 1 & 2 & 288\end{array}$

$\begin{array}{lllll}1 & 3 & 2 & 2 & 342.9\end{array}$

$\begin{array}{lllll}1 & 3 & 3 & 2 & 278.4\end{array}$

$\begin{array}{lllll}14 & 1 & 2 & 276.2\end{array}$

1422305.3

1432260.5
$\begin{array}{lllll}13 & 3 & 1 & 3 & 182\end{array}$
$\begin{array}{lllll}13 & 3 & 2 & 3 & 104.5\end{array}$
$\begin{array}{lllll}13 & 3 & 3 & 3 & 118.5\end{array}$
$\begin{array}{lllll}13 & 4 & 1 & 2 & 107.1\end{array}$
$\begin{array}{lllll}13 & 4 & 2 & 2 & 192.9\end{array}$
$\begin{array}{lllll}13 & 4 & 3 & 2 & 89.1\end{array}$
proc print; 
Tabela B.1: Dados do nível de colesterol.

\begin{tabular}{|c|c|c|c|c|c|c|c|c|c|}
\hline Nome & Data & Exam. & Colesterol & Trim. & Nome & Data & Exam. & Colest. & Trim. \\
\hline 1 & $13 / 05 / 1996$ & $\mathrm{CS}$ & 180,6 & 1 & 4 & 06/01/1995 & CS & 235,2 & 1 \\
\hline 1 & $15 / 05 / 1996$ & CS & 206,9 & 1 & 4 & 08/01/1995 & $\mathrm{CS}$ & 288,1 & 1 \\
\hline 1 & $17 / 05 / 1996$ & CS & 234,8 & 1 & 4 & $17 / 01 / 1995$ & $\mathrm{CS}$ & 232 & 1 \\
\hline 1 & $12 / 08 / 1996$ & CS & 171,3 & 2 & 4 & 28/04/1995 & CS & 199,9 & 2 \\
\hline 1 & $16 / 08 / 1996$ & CS & 174,3 & 2 & 4 & $02 / 05 / 1995$ & $\mathrm{CS}$ & 122,2 & 2 \\
\hline 1 & 20/08/1996 & CS & 185,7 & 2 & 4 & $04 / 05 / 1995$ & CS & 228,2 & 2 \\
\hline 1 & 09/11/1996 & $\mathrm{SU}$ & 288 & 3 & 4 & $20 / 07 / 1995$ & KL & 345,5 & 3 \\
\hline 1 & 13/11/1996 & $\mathrm{SU}$ & 342,9 & 3 & 4 & $27 / 07 / 1995$ & $\mathrm{KL}$ & 196,5 & 3 \\
\hline 1 & $17 / 11 / 1996$ & $\mathrm{SU}$ & 278,4 & 3 & 4 & 01/08/1995 & $\mathrm{KL}$ & 365,5 & 3 \\
\hline 1 & 13/02/1997 & $\mathrm{SU}$ & 276,2 & 4 & 4 & $24 / 10 / 1995$ & $\mathrm{CS}$ & 219,7 & 4 \\
\hline 1 & $21 / 02 / 1997$ & $\mathrm{SU}$ & 305,3 & 4 & 4 & $26 / 10 / 1995$ & $\mathrm{CS}$ & 130,8 & 4 \\
\hline 1 & $23 / 02 / 1997$ & $\mathrm{SU}$ & 260,5 & 4 & 4 & $29 / 10 / 1995$ & $\mathrm{CS}$ & 228,1 & 4 \\
\hline 2 & $10 / 07 / 1996$ & CS & 287,1 & 1 & 5 & $20 / 01 / 1995$ & $\mathrm{KL}$ & 209,1 & 1 \\
\hline 2 & 13/07/1996 & CS & 322,8 & 1 & 5 & $22 / 01 / 1995$ & $\mathrm{KL}$ & 214 & 1 \\
\hline 2 & 14/07/1996 & CS & 294,8 & 1 & 5 & $24 / 01 / 1995$ & KL & 336,2 & 1 \\
\hline 2 & $20 / 10 / 1996$ & KL & 241,1 & 2 & 5 & $30 / 04 / 1995$ & KL & 164 & 2 \\
\hline 2 & $22 / 10 / 1996$ & KL & 208,2 & 2 & 5 & $02 / 05 / 1995$ & KL & 174,6 & 2 \\
\hline 2 & $23 / 10 / 1996$ & KL & 258,4 & 2 & 5 & 04/05/1995 & $\mathrm{KL}$ & 229,1 & 2 \\
\hline 2 & $15 / 01 / 1997$ & KL & 223,3 & 3 & 5 & $30 / 07 / 1995$ & $\mathrm{CS}$ & 175,6 & 3 \\
\hline 2 & 17/01/1997 & KL & 213,1 & 3 & 5 & 06/08/1995 & $\mathrm{CS}$ & 153,7 & 3 \\
\hline 2 & $22 / 01 / 1997$ & KL & 255,3 & 3 & 5 & 08/08/1995 & $\mathrm{CS}$ & 176,9 & 3 \\
\hline 2 & 04/04/1997 & SU & 304,2 & 4 & 5 & $21 / 10 / 1995$ & SU & 198,5 & 4 \\
\hline 2 & 08/04/1997 & $\mathrm{SU}$ & 305,8 & 4 & 5 & $22 / 10 / 1995$ & $\mathrm{SU}$ & 216,8 & 4 \\
\hline 2 & $14 / 04 / 1997$ & $\mathrm{SU}$ & 244,5 & 4 & 5 & $27 / 10 / 1995$ & $\mathrm{SU}$ & 178,1 & 4 \\
\hline 3 & $22 / 12 / 1994$ & $\mathrm{KL}$ & 146 & 1 & 6 & $03 / 05 / 1995$ & $\mathrm{CS}$ & 244,6 & 1 \\
\hline 3 & 28/12/1994 & $\mathrm{KL}$ & 144,6 & 1 & 6 & $07 / 05 / 1995$ & $\mathrm{CS}$ & 262,6 & 1 \\
\hline 3 & 03/01/1995 & $\mathrm{KL}$ & 45,6 & 1 & 6 & 08/05/1995 & $\mathrm{CS}$ & 275,3 & 1 \\
\hline 3 & 09/04/1995 & KL & 141 & 2 & 6 & $22 / 07 / 1995$ & CS & 248,1 & 2 \\
\hline 3 & $12 / 04 / 1995$ & KL & 183,6 & 2 & 6 & $25 / 07 / 1995$ & $\mathrm{CS}$ & 308,8 & 2 \\
\hline 3 & $18 / 04 / 1995$ & KL & 165,6 & 2 & 6 & 06/08/1995 & $\mathrm{CS}$ & 235 & 2 \\
\hline 3 & 09/07/1995 & $\mathrm{SU}$ & 129 & 3 & 6 & $16 / 10 / 1995$ & $\mathrm{SU}$ & 318,2 & 3 \\
\hline 3 & 11/07/1995 & $\mathrm{SU}$ & 151,3 & 3 & 6 & $18 / 10 / 1995$ & $\mathrm{SU}$ & 312,7 & 3 \\
\hline 3 & $14 / 07 / 1995$ & $\mathrm{SU}$ & 110,6 & 3 & 6 & $28 / 10 / 1995$ & $\mathrm{SU}$ & 352,7 & 3 \\
\hline 3 & 01/10/1995 & CS & 179,4 & 4 & 6 & $15 / 01 / 1996$ & $\mathrm{SU}$ & 289 & 4 \\
\hline 3 & 04/10/1995 & CS & 265,3 & 4 & 6 & $18 / 01 / 1996$ & $\mathrm{SU}$ & 235,7 & 4 \\
\hline 3 & $12 / 10 / 1995$ & $\mathrm{CS}$ & 192 & 4 & 6 & $27 / 01 / 1996$ & $\mathrm{SU}$ & 281,6 & 4 \\
\hline
\end{tabular}


Tabela B.2: Continuação: dados do nível de colesterol.

\begin{tabular}{|c|c|c|c|c|c|c|c|c|c|}
\hline Nome & Data & Exam. & Colesterol & Trim. & Nome & Data & Exam. & Colest. & Trim. \\
\hline 7 & $25 / 05 / 1995$ & $\mathrm{CS}$ & 277 & 1 & 11 & $08 / 01 / 1996$ & $\mathrm{SU}$ & 133,6 & 1 \\
\hline 7 & $31 / 05 / 1995$ & CS & 265,9 & 1 & 11 & 09/01/1996 & SU & 164,7 & 1 \\
\hline 7 & 04/06/1995 & CS & 305,3 & 1 & 11 & 13/01/1996 & SU & 179,7 & 1 \\
\hline 7 & 09/08/1995 & CS & 288 & 2 & 11 & $30 / 03 / 1996$ & KL & 95,1 & 2 \\
\hline 7 & $12 / 08 / 1995$ & CS & 322,2 & 2 & 11 & $31 / 03 / 1996$ & $\mathrm{KL}$ & 100,2 & 2 \\
\hline 7 & 20/08/1995 & CS & 349 & 2 & 11 & 02/04/1996 & $\mathrm{KL}$ & 84,3 & 2 \\
\hline 7 & 05/11/1995 & CS & 169,3 & 3 & 11 & $28 / 06 / 1996$ & $\mathrm{SU}$ & 169,3 & 3 \\
\hline 7 & $10 / 11 / 1995$ & CS & 229,6 & 3 & 11 & 01/07/1996 & $\mathrm{SU}$ & 173,4 & 3 \\
\hline 7 & $16 / 11 / 1995$ & CS & 221,5 & 3 & 11 & 07/07/1996 & $\mathrm{SU}$ & 155 & 3 \\
\hline 7 & 06/02/1996 & $\mathrm{SU}$ & 245,8 & 4 & 11 & 05/10/1996 & $\mathrm{SU}$ & 110,9 & 4 \\
\hline 7 & 09/02/1996 & $\mathrm{SU}$ & 300,9 & 4 & 11 & $11 / 10 / 1996$ & $\mathrm{SU}$ & 167 & 4 \\
\hline 7 & 19/02/1996 & $\mathrm{SU}$ & 246,7 & 4 & 11 & $14 / 10 / 1996$ & $\mathrm{SU}$ & 168,3 & 4 \\
\hline 8 & $31 / 08 / 1995$ & $\mathrm{SU}$ & 156,6 & 1 & 12 & $06 / 02 / 1996$ & $\mathrm{SU}$ & 184,6 & 1 \\
\hline 8 & 04/09/1995 & $\mathrm{SU}$ & 272,5 & 1 & 12 & $16 / 02 / 1996$ & $\mathrm{SU}$ & 223,8 & 1 \\
\hline 8 & 08/09/1995 & $\mathrm{SU}$ & 175,4 & 1 & 12 & $18 / 02 / 1996$ & $\mathrm{SU}$ & 159,7 & 1 \\
\hline 8 & $18 / 12 / 1995$ & KL & 243,4 & 2 & 12 & 09/05/1996 & $\mathrm{KL}$ & 282 & 2 \\
\hline 8 & 19/12/1995 & $\mathrm{KL}$ & 222,7 & 2 & 12 & $10 / 05 / 1996$ & $\mathrm{KL}$ & 167 & 2 \\
\hline 8 & $22 / 12 / 1995$ & $\mathrm{KL}$ & 306,1 & 2 & 12 & $13 / 05 / 1996$ & $\mathrm{KL}$ & 235,5 & 2 \\
\hline 8 & 03/03/1996 & $\mathrm{SU}$ & 211,8 & 3 & 12 & 05/08/1996 & $\mathrm{SU}$ & 305,2 & 3 \\
\hline 8 & 08/03/1996 & $\mathrm{SU}$ & 171,1 & 3 & 12 & 08/08/1996 & SU & 199,3 & 3 \\
\hline 8 & 09/03/1996 & $\mathrm{SU}$ & 131,9 & 3 & 12 & $18 / 08 / 1996$ & $\mathrm{SU}$ & 227,7 & 3 \\
\hline 8 & $31 / 05 / 1996$ & $\mathrm{SU}$ & 154,9 & 4 & 12 & 08/11/1996 & $\mathrm{SU}$ & 178,2 & 4 \\
\hline 8 & 09/06/1996 & $\mathrm{SU}$ & 181,5 & 4 & 12 & 10/11/1996 & $\mathrm{SU}$ & 177,3 & 4 \\
\hline 8 & $13 / 06 / 1996$ & $\mathrm{SU}$ & 153,3 & 4 & 12 & $14 / 11 / 1996$ & $\mathrm{SU}$ & 242,8 & 4 \\
\hline 9 & $17 / 09 / 1995$ & $\mathrm{KL}$ & 223,8 & 1 & 13 & $01 / 03 / 1996$ & $\mathrm{SU}$ & 166,1 & 1 \\
\hline 9 & 21/09/1995 & $\mathrm{KL}$ & 218,9 & 1 & 13 & 04/03/1996 & $\mathrm{SU}$ & 118,1 & 1 \\
\hline 9 & $22 / 09 / 1995$ & $\mathrm{KL}$ & 194,8 & 1 & 13 & 09/03/1996 & $\mathrm{SU}$ & 142 & 1 \\
\hline 9 & $17 / 12 / 1995$ & $\mathrm{KL}$ & 233,3 & 2 & 13 & $20 / 05 / 1996$ & $\mathrm{KL}$ & 110,9 & 2 \\
\hline 9 & $20 / 12 / 1995$ & KL & 299,7 & 2 & 13 & $21 / 05 / 1996$ & KL & 115,7 & 2 \\
\hline 9 & $22 / 12 / 1995$ & $\mathrm{KL}$ & 307,7 & 2 & 13 & $24 / 05 / 1996$ & $\mathrm{KL}$ & 99,2 & 2 \\
\hline 9 & 04/03/1996 & $\mathrm{SU}$ & 326,1 & 3 & 13 & $26 / 08 / 1996$ & $\mathrm{KL}$ & 182 & 3 \\
\hline 9 & 08/03/1996 & $\mathrm{SU}$ & 414,8 & 3 & 13 & 28/08/1996 & $\mathrm{KL}$ & 104,5 & 3 \\
\hline 9 & 12/03/1996 & $\mathrm{SU}$ & 381,7 & 3 & 13 & 29/08/1996 & $\mathrm{KL}$ & 118,5 & 3 \\
\hline 9 & $30 / 05 / 1996$ & $\mathrm{SU}$ & 459,1 & 4 & 13 & $22 / 11 / 1996$ & $\mathrm{SU}$ & 107,1 & 4 \\
\hline 9 & 09/06/1996 & $\mathrm{SU}$ & 271,3 & 4 & 13 & $24 / 11 / 1996$ & $\mathrm{SU}$ & 192,9 & 4 \\
\hline 9 & $12 / 06 / 1996$ & $\mathrm{SU}$ & 309 & 4 & 13 & $27 / 11 / 1996$ & $\mathrm{SU}$ & 89,1 & 4 \\
\hline 10 & $19 / 10 / 1995$ & $\mathrm{KL}$ & 292,7 & 1 & & & & & \\
\hline 10 & $22 / 10 / 1995$ & $\mathrm{KL}$ & 284,5 & 1 & & & & & \\
\hline 10 & 26/10/1995 & $\mathrm{KL}$ & 229,2 & 1 & & & & & \\
\hline 10 & 08/01/1996 & $\mathrm{SU}$ & 183,2 & 2 & & & & & \\
\hline 10 & 10/01/1996 & $\mathrm{SU}$ & 251,5 & 2 & & & & & \\
\hline 10 & 19/01/1996 & $\mathrm{SU}$ & 201,8 & 2 & & & & & \\
\hline 10 & 05/04/1996 & CS & 226,5 & 3 & & & & & \\
\hline 10 & 09/04/1996 & $\mathrm{CS}$ & 249,4 & 3 & & & & & \\
\hline 10 & $15 / 04 / 1996$ & CS & 236,2 & 3 & & & & & \\
\hline 10 & 10/07/1996 & $\mathrm{CS}$ & 225 & 4 & & & & & \\
\hline 10 & $13 / 07 / 1996$ & $\mathrm{CS}$ & 257 & 4 & & & & & \\
\hline 10 & $15 / 07 / 1996$ & CS & 215,4 & 4 & & & & & \\
\hline
\end{tabular}



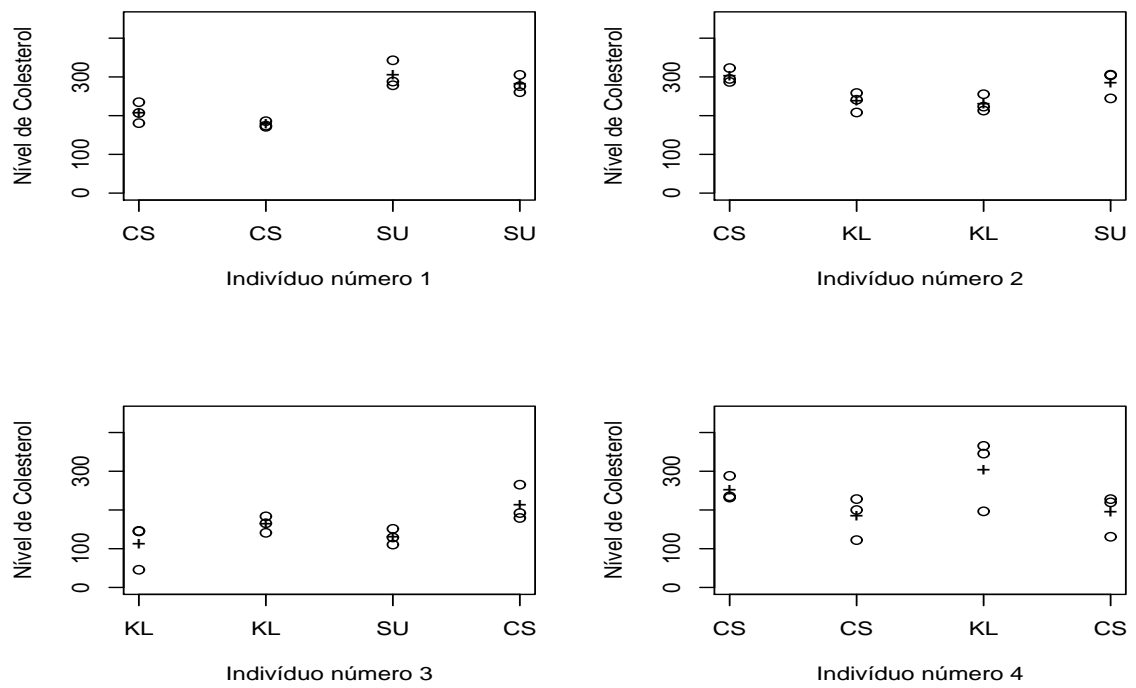

Figura B.1: Dados das medições de Colesterol dos indivíduos 1, 2, 3 e 4.
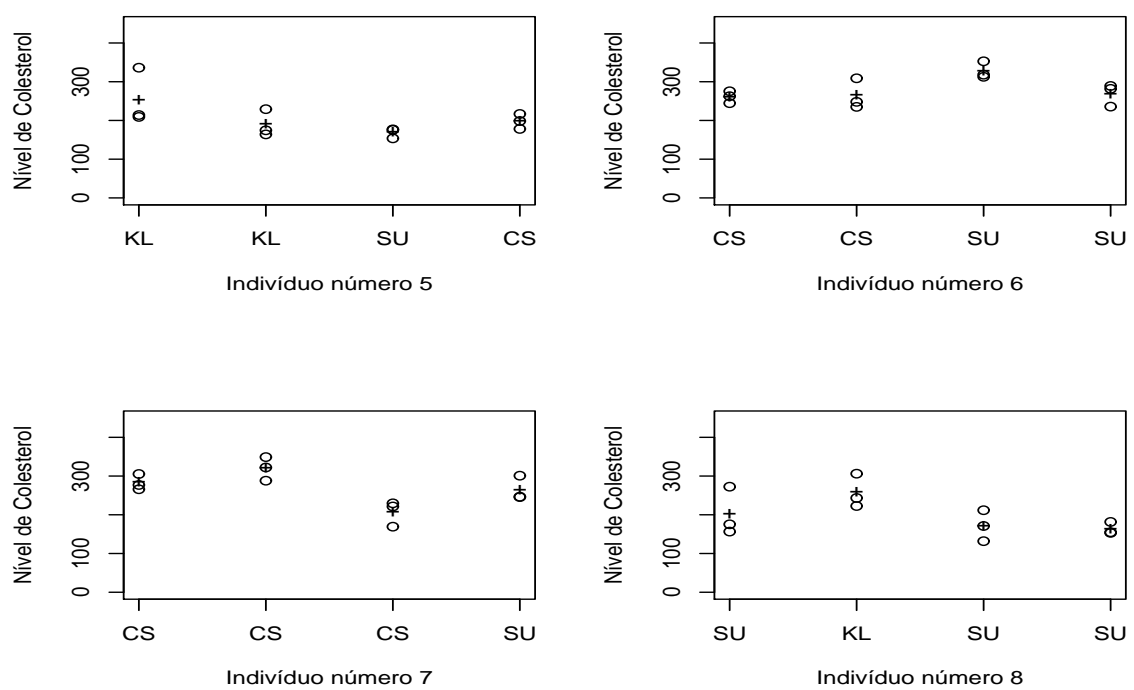

Figura B.2: Dados das medições de Colesterol dos indivíduos 5, 6, 7 e 8. 

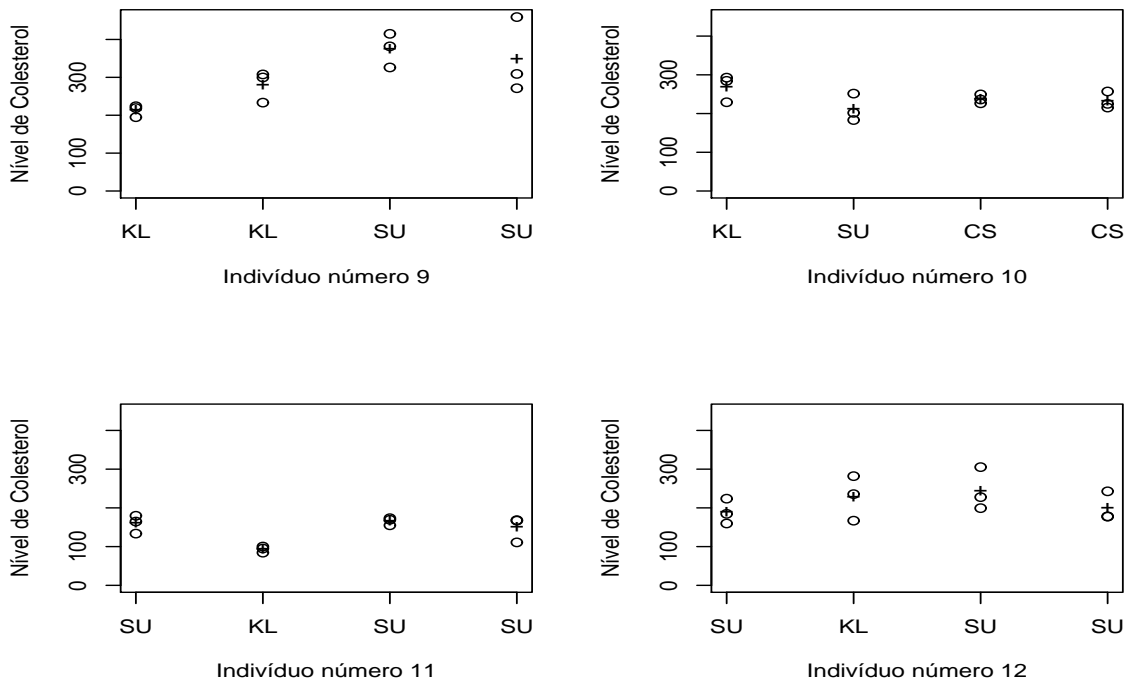

Figura B.3: Dados das medições de Colesterol dos indivíduos 9, 10, 11 e 12. 


\section{Apêndice $\mathrm{C}$}

\section{Códigos $\mathrm{R}$ do Capítulo 3}

\section{C.1 Código R Seção 3.2}

require (combinat)

require (xtable)

\#DADOS E CONSTANTES DE ENCOLHIMENTO

$\mathrm{N}=3$

$\mathrm{n}=2$

combNn $=$ choose $(\mathrm{N}, \mathrm{n})$

Factn=factorial $(n)$

\#GERAÇÃO DOS INDICES AMOSTRAIS

$\mathrm{g}<-\operatorname{combn}(1: \mathrm{N}, \mathrm{n})$

$\mathrm{C}<-\mathrm{D}<-$ matrix $(0$, combNn*Factn, $\mathrm{n})$

for ( $j$ in $1:$ combNn) \{

amostra<-permn $(g[, j])$

for ( $i$ in $1:$ Factn) \{

$a<-F a c t n *(j-1)+i$

$D[a]<,-\operatorname{amostra}[[i]]$

\}\}

\#\#99 VEZES GAMMA

\#VALORES LATENTES DAS UNIDADES DA POPULAÇÃO

$\mathrm{y}<-\mathrm{c}(10,3,2)$

gamma<-var $(\mathrm{y})$

var_se<-c $(1,100,4)$

var_e<-gamma

\#CENARIOS DE SIMULAÇÃO 


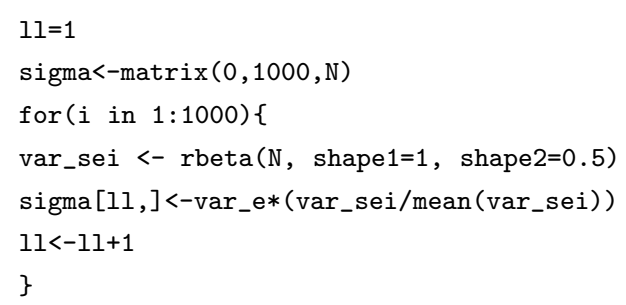




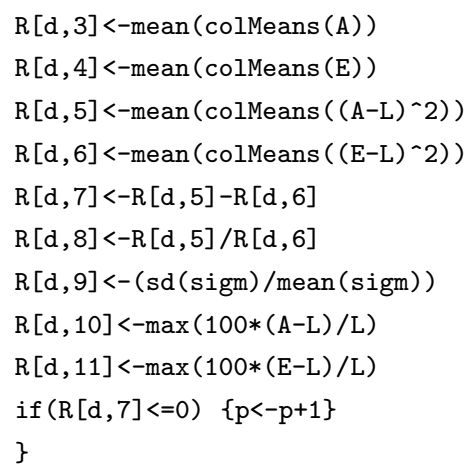

\section{C.2 Resultados da Simulação para $N=3$ e $n=2$}

Tabela C.1: Estatísticas descritivas para os quocientes $\operatorname{EQM}\left[\hat{Q}_{i}^{(1)}\right] / \operatorname{EMQ}\left[\hat{Q}_{i}^{(2)}\right]$ sob erros de medida endógenos com distribuição Uniforme para $N=3$ e $n=2$

\begin{tabular}{c|c|c|c|c|c|c}
\hline $\bar{\sigma}^{2} / \gamma^{2}$ & Mínimo & $Q_{1}$ & Mediana & Média & $Q_{3}$ & Máximo \\
\hline 19 & 0,127 & 0,789 & 0,915 & 0,864 & 0,980 & 1,000 \\
4 & 0,416 & 0,844 & 0,938 & 0,899 & 0,987 & 1,000 \\
1 & 0,524 & 0,914 & 0,969 & 0,943 & 0,993 & 1,005 \\
$1 / 4$ & 0,781 & 0,969 & 0,990 & 0,980 & 0,999 & 1,008 \\
$1 / 19$ & 0,947 & 0,993 & 0,998 & 0,995 & 1,000 & 1,004 \\
$1 / 99$ & 0,988 & 0,998 & 1,000 & 0,999 & 1,000 & 1,002 \\
\hline
\end{tabular}


Figura C.1: Quocientes $\operatorname{EQM}\left[\hat{Q}_{i}^{(1)}\right] / \operatorname{EMQ}\left[\hat{Q}_{i}^{(2)}\right]$ sob erros de medida endógenos com distribuição Normal para $N=3$ e $n=2$
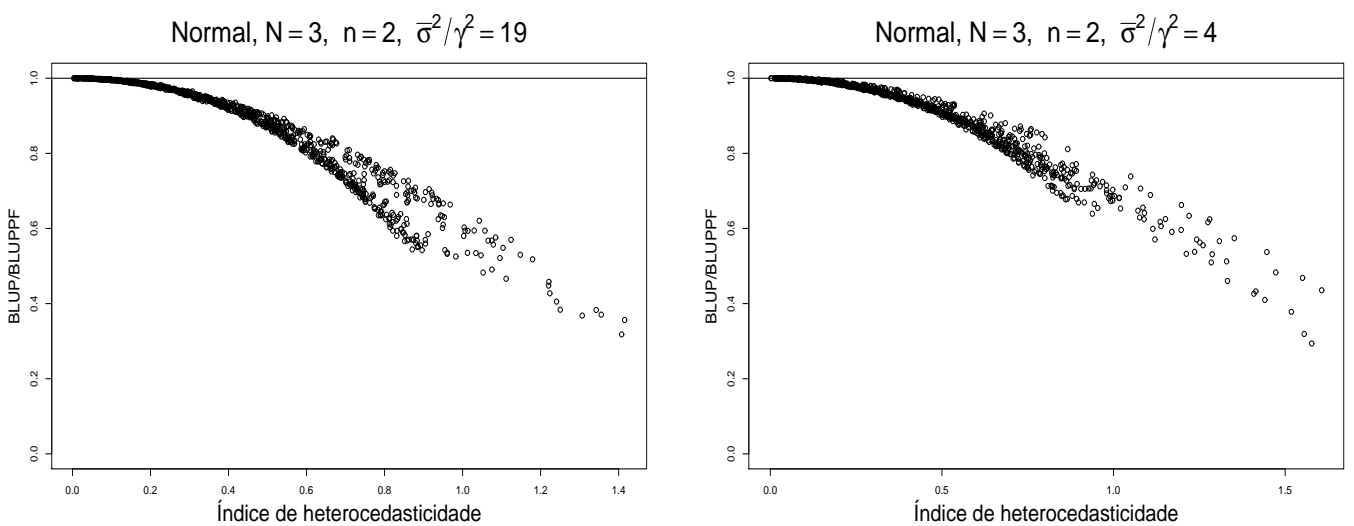

Normal, $\mathrm{N}=3, \mathrm{n}=2, \bar{\sigma}^{2} / \gamma^{2}=1$

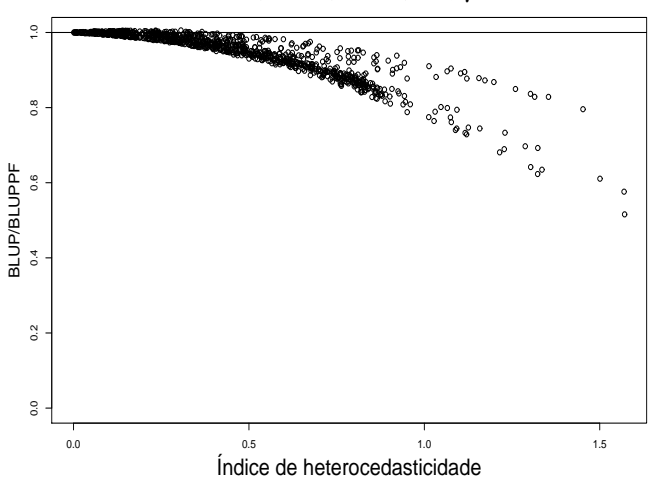

Normal, $N=3, n=2, \bar{\sigma}^{2} / \gamma^{2}=1 / 4$

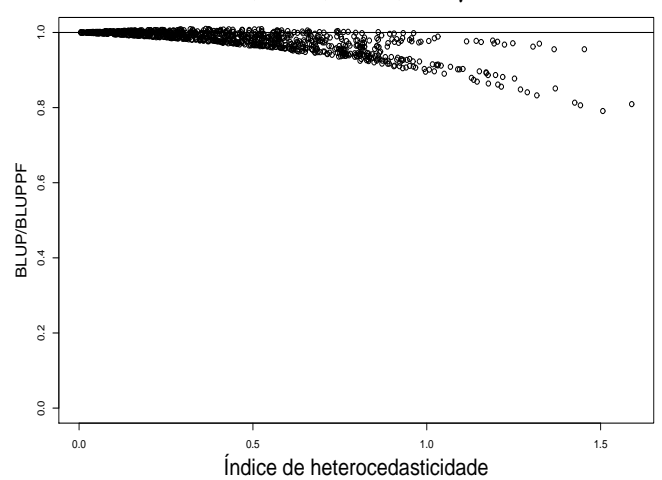

Normal, $\mathrm{N}=3, \mathrm{n}=2, \bar{\sigma}^{2} / \gamma^{2}=1 / 19$
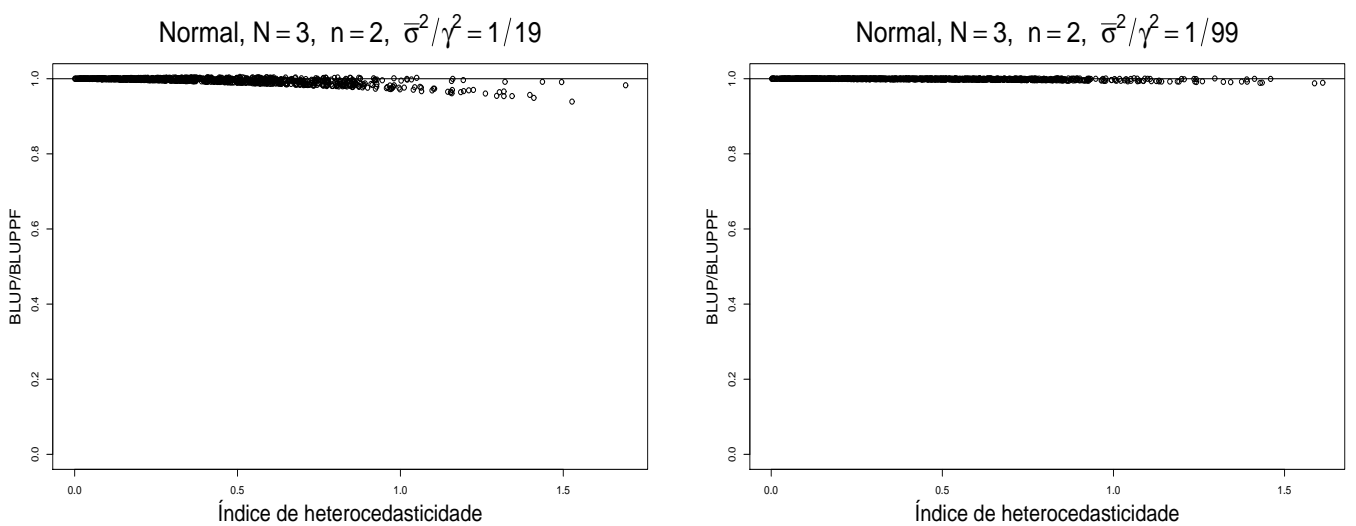
Figura C.2: Quocientes $\operatorname{EQM}\left[\hat{Q}_{i}^{(1)}\right] / \operatorname{EMQ}\left[\hat{Q}_{i}^{(2)}\right]$ sob erros de medida endógenos com distribuição Beta simétrica para $N=3$ e $n=2$
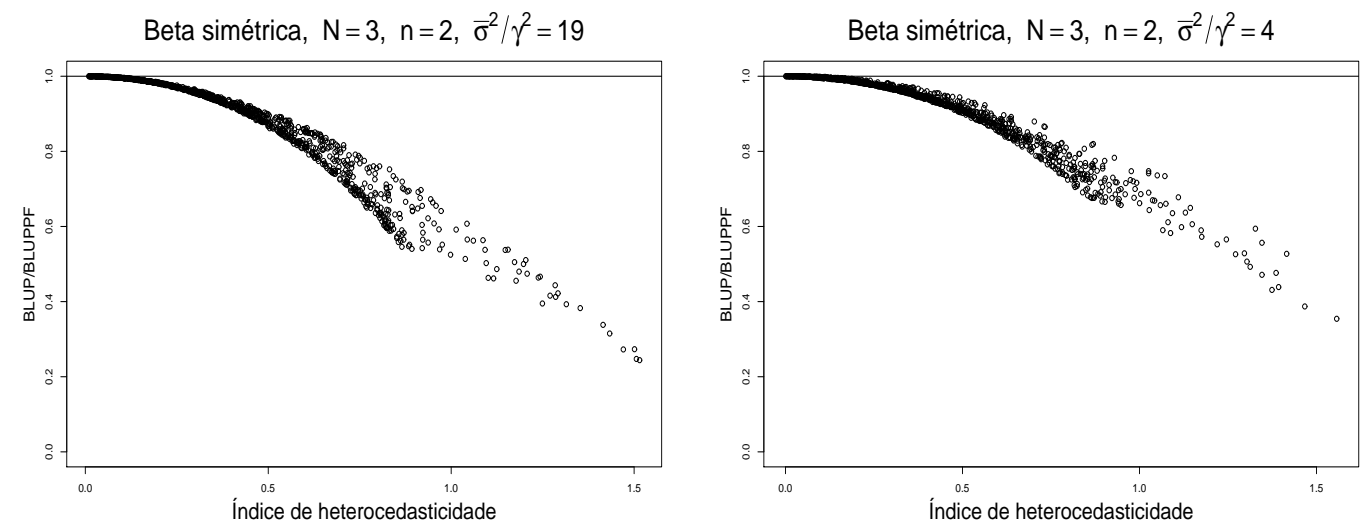

Beta simétrica, $\mathrm{N}=3, \mathrm{n}=2, \bar{\sigma}^{2} / \gamma^{2}=1$

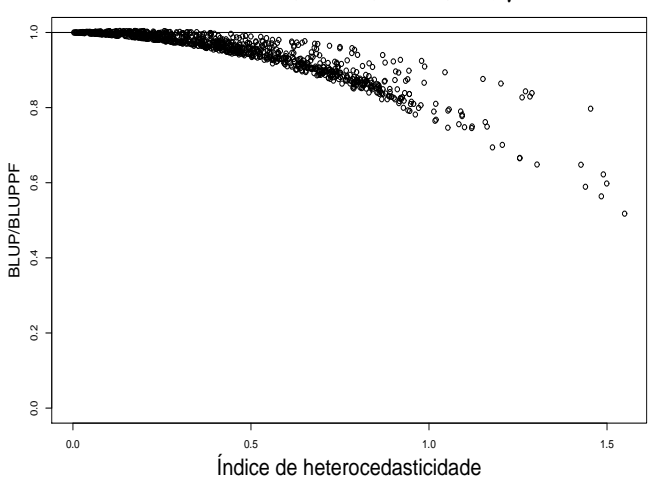

Beta simétrica, $\mathrm{N}=3, \mathrm{n}=2, \bar{\sigma}^{2} / \gamma^{2}=1 / 4$

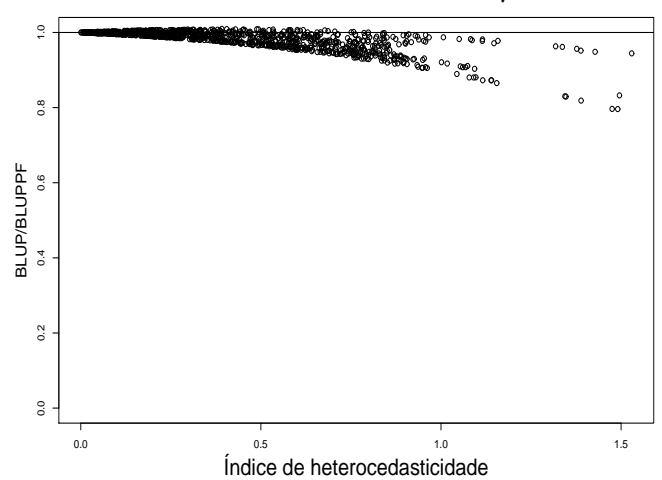

Beta simétrica, $N=3, n=2, \bar{\sigma}^{2} / \gamma^{2}=1 / 19$

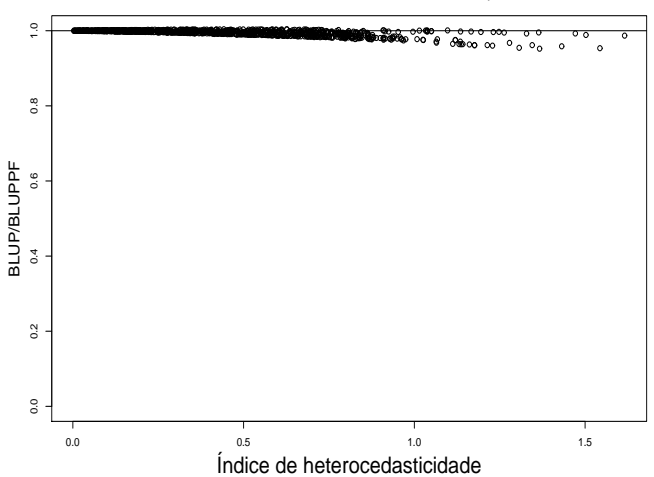

Beta simétrica, $N=3, n=2, \bar{\sigma}^{2} / \gamma^{2}=1 / 99$

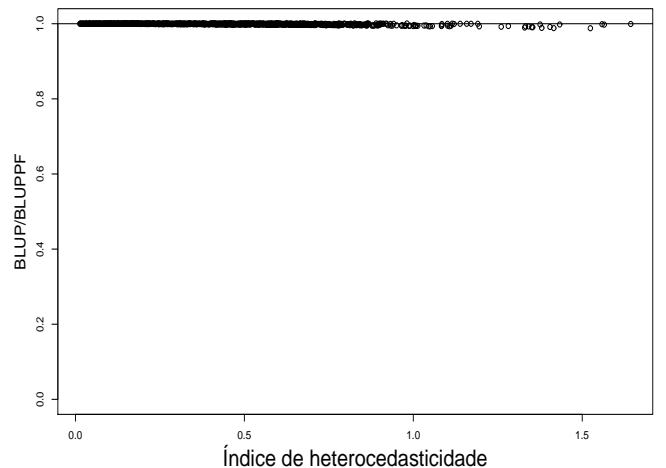


Figura C.3: Quocientes $\operatorname{EQM}\left[\hat{Q}_{i}^{(1)}\right] / \operatorname{EMQ}\left[\hat{Q}_{i}^{(2)}\right]$ sob erros de medida endógenos com distribuição Beta assimétrica para $N=3$ e $n=2$
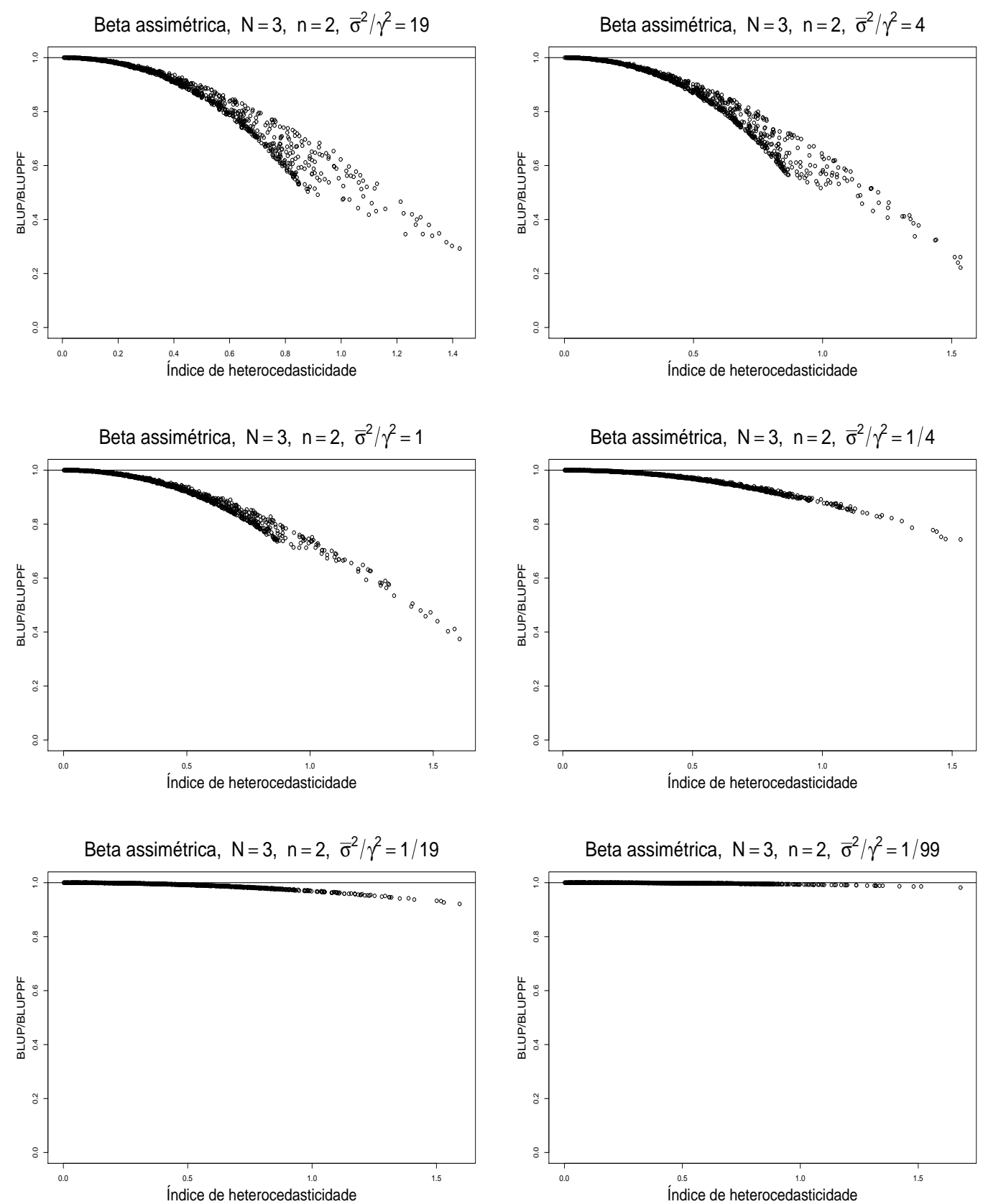
Figura C.4: Quocientes $\operatorname{EQM}\left[\hat{Q}_{i}^{(1)}\right] / \operatorname{EMQ}\left[\hat{Q}_{i}^{(2)}\right]$ sob erros de medida endógenos com distribuição Exponencial para $N=3$ e $n=2$
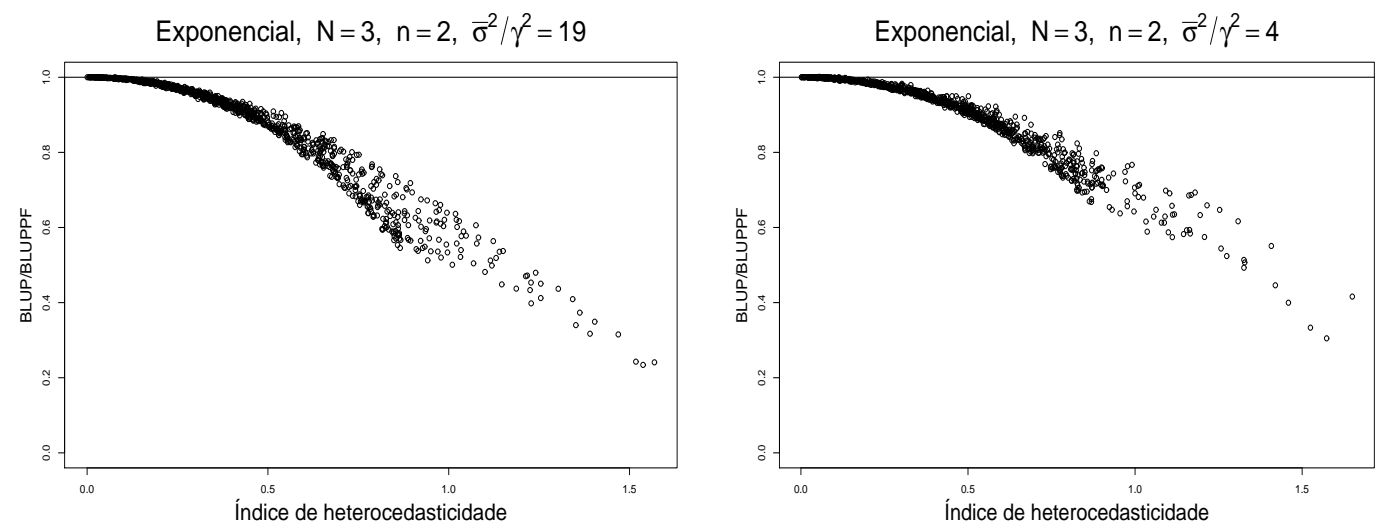

Exponencial, $\mathrm{N}=3, \mathrm{n}=2, \bar{\sigma}^{2} / \gamma^{2}=1$

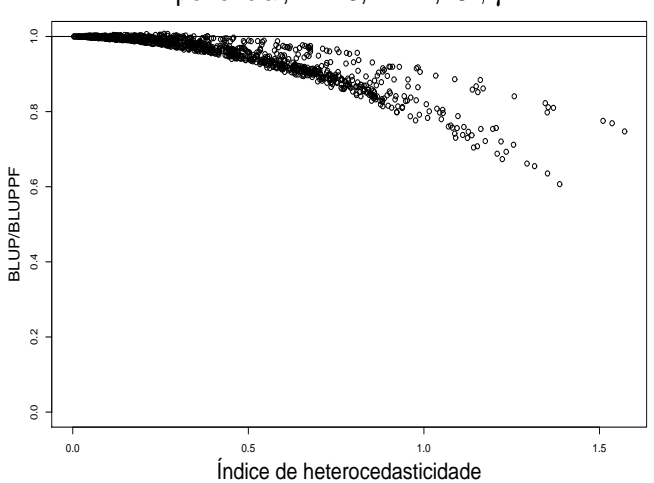

Exponencial, $\mathrm{N}=3, \mathrm{n}=2, \bar{\sigma}^{2} / \gamma^{2}=1 / 4$

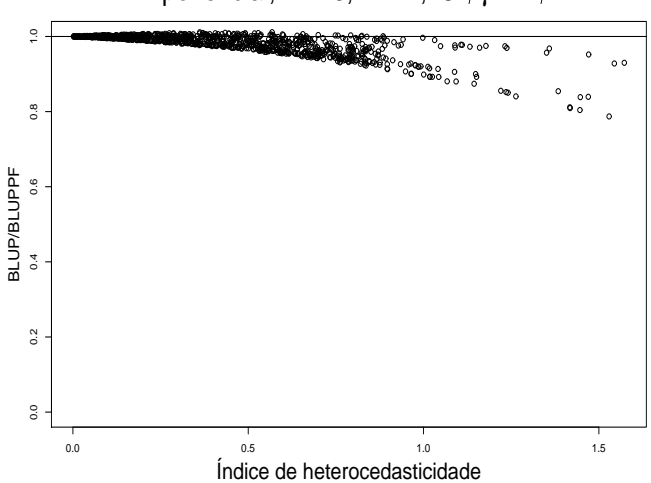

Exponencial, $\mathrm{N}=3, \mathrm{n}=2, \bar{\sigma}^{2} / \gamma^{2}=1 / 19$

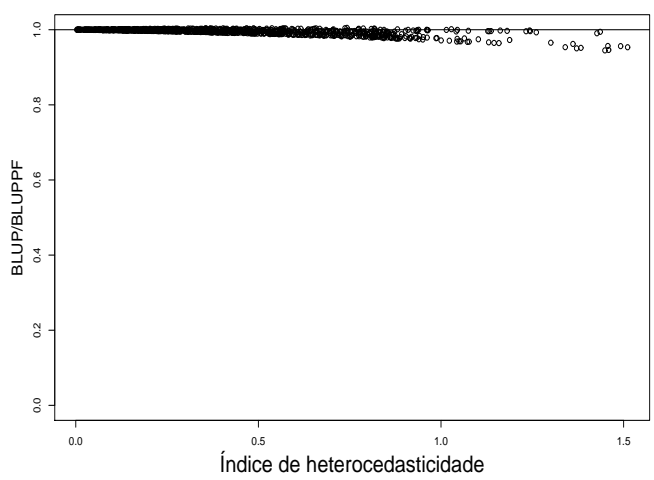

Exponencial, $N=3, n=2, \bar{\sigma}^{2} / \gamma^{2}=1 / 99$

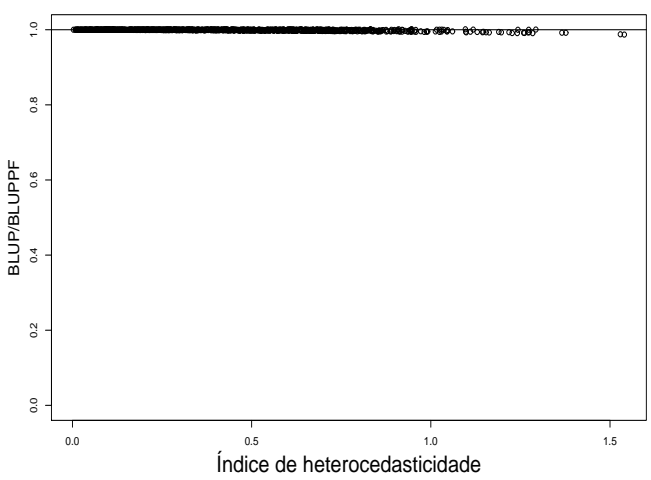


Figura C.5: Quocientes $\operatorname{EQM}\left[\hat{Q}_{i}^{(1)}\right] / \operatorname{EMQ}\left[\hat{Q}_{i}^{(2)}\right]$ sob erros de medida endógenos com distribuição Gama para $N=3$ e $n=2$
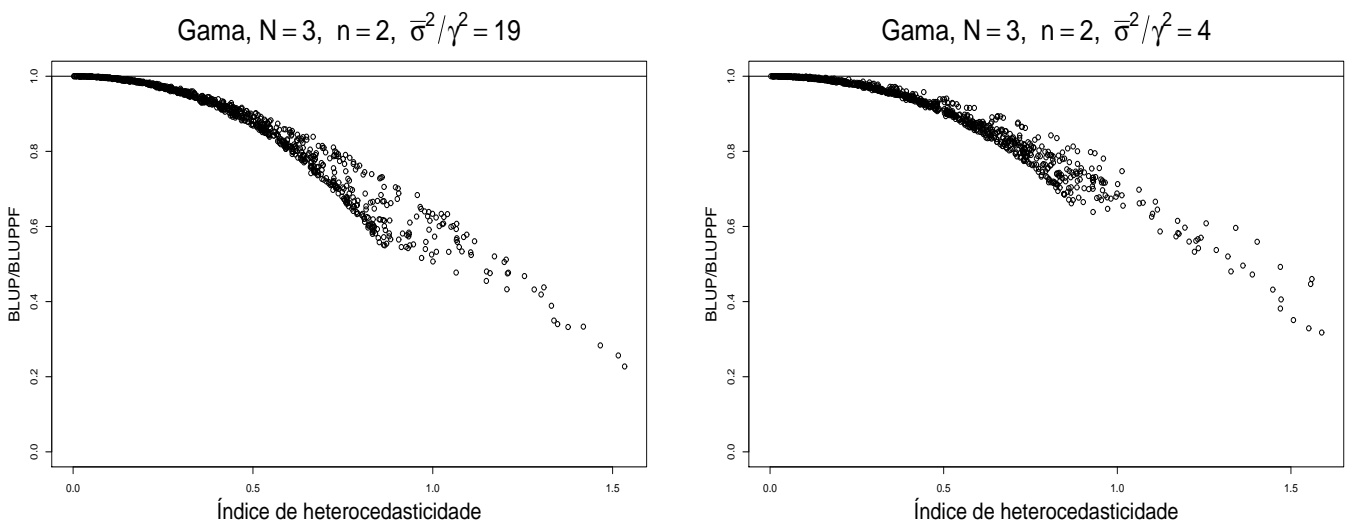

Gama, $N=3, n=2, \bar{\sigma}^{2} / \gamma^{2}=1$

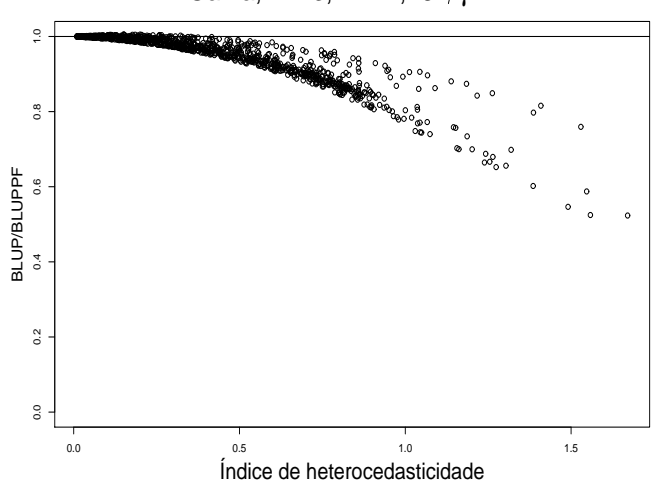

Gama, $N=3, n=2, \bar{\sigma}^{2} / \gamma^{2}=1 / 4$
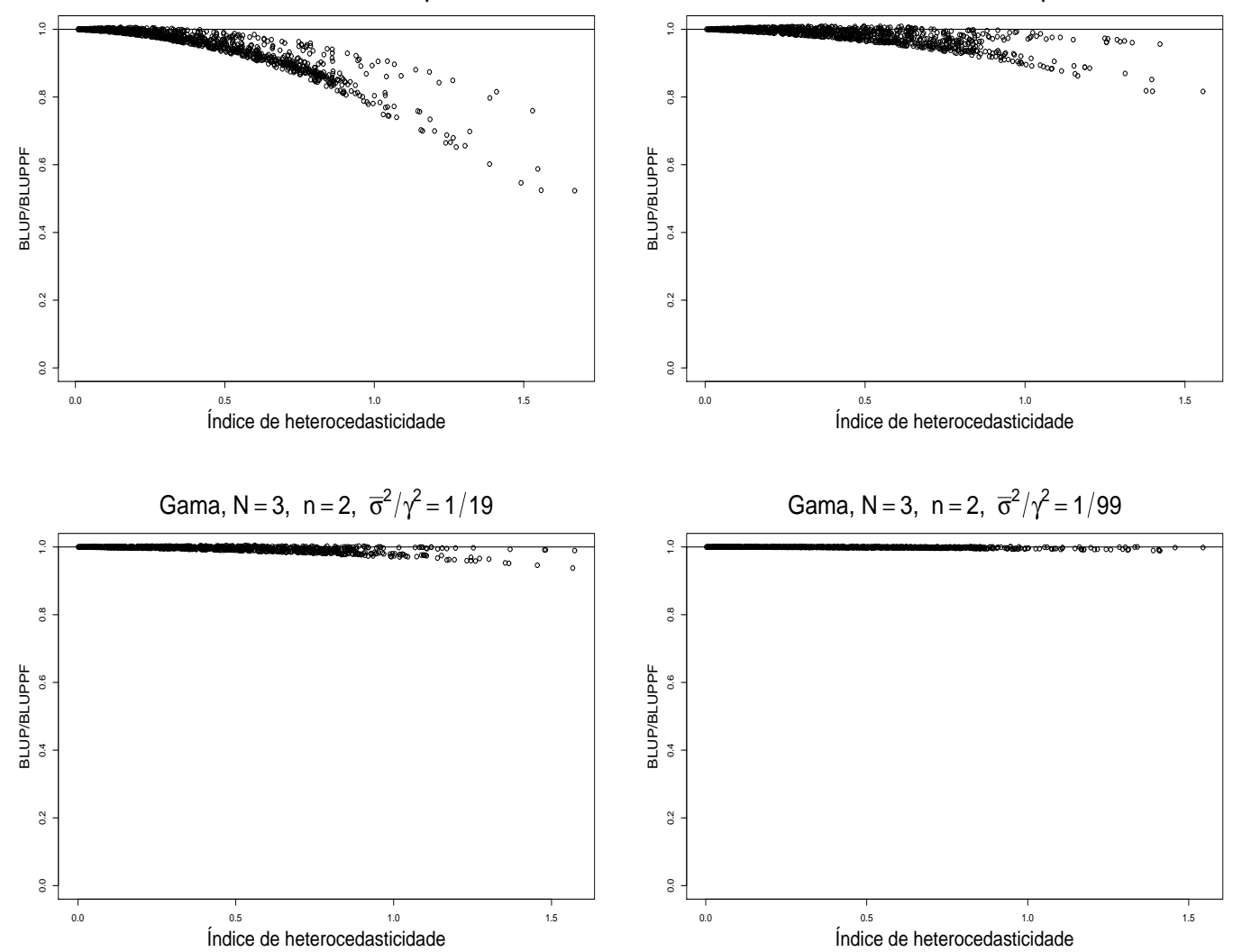
Tabela C.2: Estatísticas descritivas para os quocientes $\operatorname{EQM}\left[\hat{Q}_{i}^{(1)}\right] / \operatorname{EMQ}\left[\hat{Q}_{i}^{(2)}\right]$ sob erros de medida endógenos com distribuição Beta simétrica para $N=3$ e $n=2$

\begin{tabular}{c|c|c|c|c|c|c}
\hline $\bar{\sigma}^{2} / \gamma^{2}$ & Mínimo & $Q_{1}$ & Mediana & Média & $Q_{3}$ & Máximo \\
\hline 19 & 0,2441 & 0,8037 & 0,9186 & 0,8674 & 0,9806 & 1,000 \\
4 & 0,3543 & 0,8519 & 0,9438 & 0,9023 & 0,9833 & 1,000 \\
1 & 0,5176 & 0,9152 & 0,9683 & 0,9457 & 0,9933 & 1,004 \\
$1 / 4$ & 0,7961 & 0,9713 & 0,9908 & 0,9815 & 1,000 & 1,010 \\
$1 / 19$ & 0,9522 & 0,9921 & 0,9975 & 0,9949 & 1,000 & 1,004 \\
$1 / 99$ & 0,9880 & 0,9985 & 0,9996 & 0,999 & 1,000 & 1,001 \\
\hline
\end{tabular}

Tabela C.3: Estatísticas descritivas para os quocientes $\operatorname{EQM}\left[\hat{Q}_{i}^{(1)}\right] / \operatorname{EMQ}\left[\hat{Q}_{i}^{(2)}\right]$ sob erros de medida endógenos com distribuição Beta assimétrica para $N=3$ e $n=2$

\begin{tabular}{c|c|c|c|c|c|c}
\hline $\bar{\sigma}^{2} / \gamma^{2}$ & Mínimo & $Q_{1}$ & Mediana & Média & $Q_{3}$ & Máximo \\
\hline 19 & 0,293 & 0,768 & 0,920 & 0,859 & 0,979 & 1,000 \\
4 & 0,222 & 0,777 & 0,918 & 0,860 & 0,980 & 1,000 \\
1 & 0,375 & 0,863 & 0,946 & 0,911 & 0,984 & 1,000 \\
$1 / 4$ & 0,743 & 0,944 & 0,978 & 0,965 & 0,995 & 1,000 \\
$1 / 19$ & 0,921 & 0,986 & 0,994 & 0,990 & 0,998 & 1,000 \\
$1 / 99$ & 0,982 & 0,997 & 0,999 & 0,998 & 1,000 & 1,000 \\
\hline
\end{tabular}

Tabela C.4: Estatísticas descritivas para os quocientes $\operatorname{EQM}\left[\hat{Q}_{i}^{(1)}\right] / \operatorname{EMQ}\left[\hat{Q}_{i}^{(2)}\right]$ sob erros de medida endógenos com distribuição Exponencial para $N=3$ e $n=2$

\begin{tabular}{c|c|c|c|c|c|c}
\hline $\bar{\sigma}^{2} / \gamma^{2}$ & Mínimo & $Q_{1}$ & Mediana & Média & $Q_{3}$ & Máximo \\
\hline 19 & 0,234 & 0,787 & 0,919 & 0,860 & 0,975 & 1,000 \\
4 & 0,305 & 0,847 & 0,935 & 0,899 & 0,982 & 1,001 \\
1 & 0,607 & 0,914 & 0,968 & 0,945 & 0,993 & 1,007 \\
$1 / 4$ & 0,787 & 0,970 & 0,990 & 0,980 & 1,000 & 1,011 \\
$1 / 19$ & 0,945 & 0,993 & 0,997 & 0,995 & 1,000 & 1,005 \\
$1 / 99$ & 0,987 & 0,999 & 1,000 & 0,999 & 1,000 & 1,002 \\
\hline
\end{tabular}


Tabela C.5: Estatísticas descritivas para os quocientes $\operatorname{EQM}\left[\hat{Q}_{i}^{(1)}\right] / \operatorname{EMQ}\left[\hat{Q}_{i}^{(2)}\right]$ sob erros de medida endógenos com distribuição Gama para $N=3$ e $n=2$

\begin{tabular}{c|c|c|c|c|c|c}
\hline $\bar{\sigma}^{2} / \gamma^{2}$ & Mínimo & $Q_{1}$ & Mediana & Média & $Q_{3}$ & Máximo \\
\hline 19 & 0,227 & 0,785 & 0,919 & 0,862 & 0,979 & 1,000 \\
4 & 0,318 & 0,835 & 0,936 & 0,895 & 0,986 & 1,000 \\
1 & 0,523 & 0,915 & 0,969 & 0,945 & 0,993 & 1,005 \\
$1 / 4$ & 0,816 & 0,972 & 0,990 & 0,982 & 1,000 & 1,009 \\
$1 / 19$ & 0,938 & 0,992 & 0,998 & 0,995 & 1,000 & 1,005 \\
$1 / 99$ & 0,989 & 0,998 & 1,000 & 0,999 & 1,000 & 1,001 \\
\hline
\end{tabular}


Apêndice D

Resultados da Simulação para outros valores de $N$ e $n$ 
Tabela D.1: Número de populações em que o preditor $\hat{Q}_{i}^{(1)}$ tem menor erro quadrático médio que o preditor $\hat{Q}_{i}^{(2)}$ de um total de 500 populações simuladas com valores latentes seguindo uma distribuição assimétrica à esquerda

\begin{tabular}{|c|c|c|c|c|c|c|c|c|}
\hline$N$ & $n$ & $\bar{\sigma}^{2} / \gamma^{2}$ & Normal & Uniforme & Beta Simétrica & Beta Assimétrica & Exponencial & Gama \\
\hline \multirow{6}{*}{50} & \multirow{6}{*}{5} & 19 & 500 & 500 & 500 & 500 & 500 & 500 \\
\hline & & 4 & 500 & 500 & 500 & 500 & 500 & 500 \\
\hline & & 1 & 492 & 493 & 495 & 494 & 488 & 493 \\
\hline & & $1 / 4$ & 476 & 470 & 482 & 470 & 476 & 474 \\
\hline & & $1 / 19$ & 448 & 462 & 454 & 457 & 451 & 453 \\
\hline & & $1 / 99$ & 444 & 423 & 420 & 437 & 422 & 413 \\
\hline \multirow{6}{*}{50} & \multirow{6}{*}{10} & 19 & 500 & 500 & 500 & 500 & 500 & 500 \\
\hline & & 4 & 498 & 500 & 500 & 500 & 500 & 500 \\
\hline & & 1 & 490 & 489 & 491 & 495 & 487 & 488 \\
\hline & & $1 / 4$ & 465 & 470 & 475 & 467 & 472 & 467 \\
\hline & & $1 / 19$ & 450 & 436 & 457 & 450 & 448 & 462 \\
\hline & & $1 / 99$ & 431 & 443 & 445 & 442 & 439 & 433 \\
\hline \multirow{6}{*}{50} & \multirow{6}{*}{25} & 19 & 500 & 500 & 500 & 500 & 500 & 500 \\
\hline & & 4 & 497 & 499 & 498 & 495 & 499 & 499 \\
\hline & & 1 & 479 & 485 & 482 & 485 & 482 & 483 \\
\hline & & $1 / 4$ & 464 & 461 & 468 & 470 & 469 & 466 \\
\hline & & $1 / 19$ & 455 & 457 & 453 & 464 & 449 & 438 \\
\hline & & $1 / 99$ & 430 & 439 & 447 & 442 & 435 & 441 \\
\hline \multirow{6}{*}{100} & \multirow{6}{*}{10} & 19 & $\overline{500}$ & 500 & 500 & $\overline{500}$ & $\overline{500}$ & 2500 \\
\hline & & 4 & 500 & 500 & 500 & 500 & 500 & 500 \\
\hline & & 1 & 499 & 498 & 496 & 497 & 499 & 497 \\
\hline & & $1 / 4$ & 488 & 494 & 482 & 492 & 486 & 481 \\
\hline & & $1 / 19$ & 474 & 477 & 469 & 480 & 476 & 469 \\
\hline & & $1 / 99$ & 453 & 465 & 459 & 461 & 442 & 453 \\
\hline \multirow{6}{*}{100} & \multirow{6}{*}{30} & 19 & 500 & 500 & 500 & 500 & 500 & 500 \\
\hline & & 4 & 500 & 500 & 498 & 499 & 499 & 500 \\
\hline & & 1 & 494 & 495 & 488 & 492 & 494 & 495 \\
\hline & & $1 / 4$ & 495 & 484 & 489 & 487 & 483 & 493 \\
\hline & & $1 / 19$ & 462 & 470 & 470 & 465 & 482 & 471 \\
\hline & & $1 / 99$ & 463 & 465 & 461 & 463 & 459 & 460 \\
\hline \multirow{6}{*}{100} & \multirow{6}{*}{50} & 19 & 500 & 500 & 500 & 500 & 500 & 500 \\
\hline & & 4 & 499 & 500 & 499 & 499 & 496 & 496 \\
\hline & & 1 & 489 & 499 & 493 & 493 & 493 & 494 \\
\hline & & $1 / 4$ & 488 & 499 & 493 & 480 & 490 & 488 \\
\hline & & $1 / 19$ & 472 & 497 & 468 & 477 & 468 & 474 \\
\hline & & $1 / 99$ & 472 & 492 & 464 & 462 & 479 & 474 \\
\hline \multirow{6}{*}{300} & \multirow{6}{*}{30} & 19 & 500 & $\overline{500}$ & $\overline{500}$ & 2500 & $\overline{500}$ & 500 \\
\hline & & 4 & 500 & 499 & 500 & 500 & 500 & 500 \\
\hline & & 1 & 499 & 492 & 499 & 500 & 499 & 497 \\
\hline & & $1 / 4$ & 496 & 489 & 497 & 498 & 499 & 497 \\
\hline & & $1 / 19$ & 492 & 478 & 498 & 495 & 495 & 495 \\
\hline & & $1 / 99$ & 486 & 466 & 489 & 486 & 485 & 483 \\
\hline \multirow{6}{*}{300} & \multirow{6}{*}{50} & 19 & 500 & 500 & 500 & 500 & 500 & 500 \\
\hline & & 4 & 499 & 500 & 500 & 500 & 499 & 500 \\
\hline & & 1 & 500 & 499 & 500 & 499 & 498 & 499 \\
\hline & & $1 / 4$ & 497 & 498 & 499 & 499 & 495 & 496 \\
\hline & & $1 / 19$ & 496 & 493 & 496 & 494 & 492 & 496 \\
\hline & & $1 / 99$ & 483 & 492 & 486 & 485 & 484 & 490 \\
\hline \multirow{6}{*}{300} & & 19 & 500 & 500 & 500 & 500 & 500 & 500 \\
\hline & & 4 & 500 & 500 & 499 & 500 & 499 & 500 \\
\hline & 100 & 1 & 499 & 499 & 499 & 499 & 499 & 499 \\
\hline & & $1 / 4$ & 500 & 500 & 496 & 495 & 497 & 499 \\
\hline & & $1 / 19$ & 499 & 495 & 494 & 496 & 492 & 497 \\
\hline & & $1 / 99$ & 498 & 486 & 493 & 490 & 489 & 489 \\
\hline
\end{tabular}


Tabela D.2: Número de populações em que o preditor $\hat{Q}_{i}^{(1)}$ tem menor erro quadrático médio que o preditor $\hat{Q}_{i}^{(2)}$ de um total de 500 populações simuladas com valores latentes seguindo uma distribuição assimétrica à direita

\begin{tabular}{|c|c|c|c|c|c|c|c|c|}
\hline$N$ & $n$ & $\bar{\sigma}^{2} / \gamma^{2}$ & Normal & Uniforme & Beta Simétrica & Beta Assimétrica & Exponencial & Gama \\
\hline \multirow{6}{*}{50} & \multirow{6}{*}{5} & 19 & 500 & 500 & 500 & 500 & 499 & 500 \\
\hline & & 4 & 499 & 500 & 500 & 500 & 500 & 500 \\
\hline & & 1 & 496 & 491 & 497 & 493 & 497 & 495 \\
\hline & & $1 / 4$ & 493 & 475 & 480 & 476 & 482 & 482 \\
\hline & & $1 / 19$ & 463 & 454 & 461 & 460 & 450 & 457 \\
\hline & & $1 / 99$ & 437 & 428 & 430 & 432 & 433 & 434 \\
\hline \multirow{6}{*}{50} & \multirow{6}{*}{10} & 19 & 500 & 500 & 500 & 500 & 500 & 500 \\
\hline & & 4 & 500 & 499 & 500 & 500 & 500 & 500 \\
\hline & & 1 & 486 & 489 & 492 & 485 & 491 & 485 \\
\hline & & $1 / 4$ & 465 & 476 & 477 & 474 & 472 & 469 \\
\hline & & $1 / 19$ & 460 & 457 & 447 & 462 & 454 & 452 \\
\hline & & $1 / 99$ & 437 & 432 & 446 & 443 & 452 & 440 \\
\hline \multirow{6}{*}{50} & \multirow{6}{*}{25} & 19 & 500 & 500 & 500 & 500 & 500 & 500 \\
\hline & & 4 & 497 & 499 & 492 & 500 & 496 & 499 \\
\hline & & 1 & 484 & 481 & 488 & 485 & 484 & 482 \\
\hline & & $1 / 4$ & 470 & 478 & 464 & 464 & 476 & 464 \\
\hline & & $1 / 19$ & 459 & 452 & 456 & 455 & 448 & 459 \\
\hline & & $1 / 99$ & 427 & 435 & 451 & 454 & 433 & 452 \\
\hline \multirow{6}{*}{100} & \multirow{6}{*}{10} & 19 & $\overline{500}$ & 500 & 500 & $\overline{500}$ & $\overline{500}$ & 2500 \\
\hline & & 4 & 500 & 500 & 500 & 500 & 500 & 500 \\
\hline & & 1 & 495 & 500 & 499 & 499 & 498 & 498 \\
\hline & & $1 / 4$ & 488 & 491 & 494 & 485 & 487 & 490 \\
\hline & & $1 / 19$ & 478 & 477 & 482 & 474 & 459 & 478 \\
\hline & & $1 / 99$ & 453 & 456 & 456 & 468 & 450 & 449 \\
\hline \multirow{6}{*}{100} & \multirow{6}{*}{30} & 19 & 500 & 500 & 500 & 500 & 500 & 500 \\
\hline & & 4 & 500 & 498 & 500 & 498 & 499 & 499 \\
\hline & & 1 & 495 & 492 & 497 & 495 & 489 & 494 \\
\hline & & $1 / 4$ & 484 & 481 & 489 & 482 & 487 & 486 \\
\hline & & $1 / 19$ & 476 & 468 & 477 & 478 & 462 & 465 \\
\hline & & $1 / 99$ & 464 & 463 & 470 & 465 & 463 & 461 \\
\hline \multirow{6}{*}{100} & \multirow{6}{*}{50} & 19 & 500 & 500 & 500 & 500 & 500 & 500 \\
\hline & & 4 & 497 & 497 & 498 & 499 & 499 & 498 \\
\hline & & 1 & 496 & 497 & 492 & 491 & 488 & 493 \\
\hline & & $1 / 4$ & 488 & 488 & 491 & 483 & 484 & 486 \\
\hline & & $1 / 19$ & 473 & 473 & 469 & 471 & 474 & 472 \\
\hline & & $1 / 99$ & 467 & 464 & 476 & 463 & 457 & 464 \\
\hline \multirow{6}{*}{300} & \multirow{6}{*}{30} & 19 & 500 & $\overline{500}$ & $\overline{500}$ & 2500 & $\overline{500}$ & 500 \\
\hline & & 4 & 500 & 500 & 500 & 500 & 500 & 500 \\
\hline & & 1 & 499 & 500 & 500 & 500 & 499 & 499 \\
\hline & & $1 / 4$ & 497 & 497 & 499 & 499 & 496 & 498 \\
\hline & & $1 / 19$ & 498 & 495 & 494 & 494 & 494 & 492 \\
\hline & & $1 / 99$ & 481 & 483 & 490 & 485 & 486 & 481 \\
\hline \multirow{6}{*}{300} & \multirow{6}{*}{50} & 19 & 500 & 500 & 500 & 500 & 500 & 500 \\
\hline & & 4 & 500 & 500 & 500 & 500 & 500 & 500 \\
\hline & & 1 & 500 & 497 & 498 & 498 & 499 & 499 \\
\hline & & $1 / 4$ & 498 & 500 & 500 & 497 & 497 & 497 \\
\hline & & $1 / 19$ & 496 & 494 & 491 & 496 & 489 & 497 \\
\hline & & $1 / 99$ & 492 & 488 & 493 & 488 & 486 & 491 \\
\hline \multirow{6}{*}{300} & & 19 & 500 & 500 & 500 & 500 & 500 & 500 \\
\hline & & 4 & 500 & 500 & 500 & 500 & 500 & 500 \\
\hline & 100 & 1 & 498 & 500 & 500 & 500 & 500 & 499 \\
\hline & & $1 / 4$ & 499 & 500 & 499 & 500 & 498 & 498 \\
\hline & & $1 / 19$ & 498 & 495 & 496 & 497 & 498 & 498 \\
\hline & & $1 / 99$ & 493 & 494 & 490 & 490 & 489 & 484 \\
\hline
\end{tabular}


Tabela D.3: Valor máximo do quociente $\operatorname{EQM}\left[\hat{Q}_{i}^{(1)}\right] / \operatorname{EMQ}\left[\hat{Q}_{i}^{(2)}\right]$ com valores latentes seguindo uma distribuição assimétrica à esquerda

\begin{tabular}{|c|c|c|c|c|c|c|c|c|}
\hline$N$ & $n$ & $\overline{\sigma^{2} / \gamma^{2}}$ & Normal & Uniforme & Beta Simétrica & Beta Assimétrica & Exponencial & Gama \\
\hline \multirow{6}{*}{50} & \multirow{6}{*}{5} & 19 & 0.977 & 0.958 & 0.973 & 0.974 & 0.980 & 0.973 \\
\hline & & 4 & 0.998 & 0.981 & 0.988 & 0.976 & 0.989 & 0.980 \\
\hline & & 1 & 1.014 & 1.026 & 1.035 & 1.013 & 1.020 & 1.096 \\
\hline & & $1 / 4$ & 1.068 & 1.128 & 1.170 & 1.184 & 1.137 & 1.293 \\
\hline & & $1 / 19$ & 1.256 & 1.140 & 1.124 & 1.217 & 1.084 & 1.063 \\
\hline & & $1 / 99$ & 1.045 & 1.103 & 1.048 & 1.049 & 1.073 & 1.054 \\
\hline \multirow{6}{*}{50} & \multirow{6}{*}{10} & 19 & 0.974 & 0.955 & 0.967 & 0.983 & 0.978 & 0.968 \\
\hline & & 4 & 1.025 & 0.991 & 0.992 & 0.989 & 0.997 & 0.995 \\
\hline & & 1 & 1.050 & 1.030 & 1.072 & 1.019 & 1.058 & 1.025 \\
\hline & & $1 / 4$ & 1.089 & 1.100 & 1.160 & 1.125 & 1.090 & 1.094 \\
\hline & & $1 / 19$ & 1.130 & 1.279 & 1.344 & 1.189 & 1.272 & 1.050 \\
\hline & & $1 / 99$ & 1.091 & 1.140 & 1.031 & 1.090 & 1.142 & 1.159 \\
\hline \multirow{6}{*}{50} & \multirow{6}{*}{25} & 19 & 0.983 & 0.974 & 0.985 & 0.988 & 0.979 & 0.987 \\
\hline & & 4 & 1.020 & 1.004 & 1.022 & 1.011 & 1.016 & 1.002 \\
\hline & & 1 & 1.049 & 1.034 & 1.095 & 1.055 & 1.066 & 1.042 \\
\hline & & $1 / 4$ & 1.103 & 1.133 & 1.060 & 1.282 & 1.151 & 1.123 \\
\hline & & $1 / 19$ & 1.076 & 1.106 & 1.181 & 1.240 & 1.603 & 1.333 \\
\hline & & $1 / 99$ & 1.071 & 1.091 & 1.065 & 1.177 & 1.078 & 1.041 \\
\hline \multirow{6}{*}{100} & \multirow{6}{*}{10} & 19 & 0.984 & 0.956 & 0.974 & 0.979 & 1.000 & 0.990 \\
\hline & & 4 & 0.996 & 0.992 & 0.982 & 0.988 & 0.987 & 0.986 \\
\hline & & 1 & 1.011 & 1.004 & 1.027 & 1.013 & 1.001 & 1.019 \\
\hline & & $1 / 4$ & 1.078 & 1.010 & 1.078 & 1.054 & 1.057 & 1.052 \\
\hline & & $1 / 19$ & 1.061 & 1.071 & 1.062 & 1.107 & 1.349 & 1.065 \\
\hline & & $1 / 99$ & 1.019 & 1.095 & 1.068 & 1.026 & 1.080 & 1.177 \\
\hline \multirow{6}{*}{100} & \multirow{6}{*}{30} & 19 & 0.981 & 0.993 & 0.973 & 0.979 & 0.979 & 0.974 \\
\hline & & 4 & 1.000 & 1.000 & 1.010 & 1.003 & 1.002 & 1.000 \\
\hline & & 1 & 1.015 & 1.087 & 1.038 & 1.036 & 1.035 & 1.014 \\
\hline & & $1 / 4$ & 1.017 & 1.050 & 1.026 & 1.039 & 1.066 & 1.028 \\
\hline & & $1 / 19$ & 1.048 & 1.115 & 1.073 & 1.097 & 1.121 & 1.104 \\
\hline & & $1 / 99$ & 1.015 & 1.163 & 1.029 & 1.016 & 1.075 & 1.049 \\
\hline \multirow{6}{*}{100} & \multirow{6}{*}{50} & 19 & 0.982 & 0.983 & 0.984 & 0.987 & 0.988 & 0.989 \\
\hline & & 4 & 1.011 & 1.001 & 1.001 & 1.007 & 1.008 & 1.010 \\
\hline & & 1 & 1.034 & 1.051 & 1.013 & 1.031 & 1.064 & 1.024 \\
\hline & & $1 / 4$ & 1.050 & 1.051 & 1.019 & 1.076 & 1.061 & 1.037 \\
\hline & & $1 / 19$ & 1.080 & 1.075 & 1.043 & 1.293 & 1.134 & 1.071 \\
\hline & & $1 / 99$ & 1.099 & 1.014 & 1.102 & 1.018 & 1.087 & 1.099 \\
\hline \multirow{6}{*}{300} & \multirow{6}{*}{30} & $\bar{~} 19$ & 0.972 & 0.982 & 0.981 & 0.982 & 0.982 & 0.990 \\
\hline & & 4 & 0.991 & 0.993 & 0.996 & 0.989 & 0.993 & 0.993 \\
\hline & & 1 & 1.003 & 1.001 & 1.000 & 0.986 & 1.006 & 1.006 \\
\hline & & $1 / 4$ & 1.006 & 1.005 & 1.001 & 1.004 & 1.005 & 1.008 \\
\hline & & $1 / 19$ & 1.011 & 1.010 & 1.001 & 1.008 & 1.019 & 1.017 \\
\hline & & $1 / 99$ & 1.020 & 1.007 & 1.007 & 1.035 & 1.015 & 1.026 \\
\hline \multirow{6}{*}{300} & \multirow{6}{*}{50} & 19 & 0.980 & 0.987 & 0.985 & 0.986 & 0.988 & 0.983 \\
\hline & & 4 & 1.004 & 0.996 & 0.995 & 0.997 & 1.002 & 0.998 \\
\hline & & 1 & 0.997 & 1.001 & 0.993 & 1.008 & 1.011 & 1.001 \\
\hline & & $1 / 4$ & 1.004 & 1.007 & 1.003 & 1.001 & 1.004 & 1.014 \\
\hline & & $1 / 19$ & 1.007 & 1.011 & 1.008 & 1.010 & 1.041 & 1.004 \\
\hline & & $1 / 99$ & 1.007 & 1.018 & 1.003 & 1.009 & 1.009 & 1.025 \\
\hline \multirow{6}{*}{300} & & 19 & 0.993 & 0.990 & 0.988 & 0.991 & 0.990 & 0.991 \\
\hline & & 4 & 0.996 & 0.993 & 1.000 & 0.995 & 1.000 & 0.996 \\
\hline & 100 & 1 & 1.001 & 1.006 & 1.005 & 1.002 & 1.005 & 1.016 \\
\hline & & $1 / 4$ & 0.997 & 0.998 & 1.014 & 1.016 & 1.009 & 1.001 \\
\hline & & $1 / 19$ & 1.005 & 1.051 & 1.012 & 1.043 & 1.042 & 1.002 \\
\hline & & $1 / 99$ & 1.039 & 1.051 & 1.016 & 1.018 & 1.037 & 1.081 \\
\hline
\end{tabular}


Tabela D.4: Valor máximo do quociente $\operatorname{EQM}\left[\hat{Q}_{i}^{(1)}\right] / \operatorname{EMQ}\left[\hat{Q}_{i}^{(2)}\right]$ com valores latentes seguindo uma distribuição assimétrica à direita

\begin{tabular}{|c|c|c|c|c|c|c|c|c|}
\hline$N$ & $n$ & $\bar{\sigma}^{2} / \gamma^{2}$ & Normal & Uniforme & Beta Simétrica & Beta Assimétrica & Exponencial & Gama \\
\hline \multirow{6}{*}{50} & \multirow{6}{*}{5} & 19 & 0.976 & 0.965 & 0.975 & 0.979 & 1.006 & 0.989 \\
\hline & & 4 & 1.000 & 0.990 & 0.990 & 0.990 & 0.988 & 0.995 \\
\hline & & 1 & 1.010 & 1.043 & 1.063 & 1.039 & 1.017 & 1.029 \\
\hline & & $1 / 4$ & 1.094 & 1.166 & 1.081 & 1.128 & 1.047 & 1.157 \\
\hline & & $1 / 19$ & 1.095 & 1.164 & 1.243 & 1.326 & 1.062 & 1.355 \\
\hline & & $1 / 99$ & 1.051 & 1.034 & 1.088 & 1.125 & 1.058 & 1.259 \\
\hline \multirow{6}{*}{50} & \multirow{6}{*}{10} & 19 & 0.972 & 0.965 & 0.962 & 0.984 & 0.991 & 0.985 \\
\hline & & 4 & 0.985 & 1.006 & 0.998 & 0.993 & 0.991 & 0.994 \\
\hline & & 1 & 1.055 & 1.055 & 1.049 & 1.039 & 1.033 & 1.050 \\
\hline & & $1 / 4$ & 1.289 & 1.105 & 1.201 & 1.098 & 1.220 & 1.129 \\
\hline & & $1 / 19$ & 1.126 & 1.145 & 1.312 & 1.179 & 1.083 & 1.369 \\
\hline & & $1 / 99$ & 1.157 & 1.078 & 1.068 & 1.217 & 1.072 & 1.198 \\
\hline \multirow{6}{*}{50} & \multirow{6}{*}{25} & 19 & 0.983 & 0.980 & 0.983 & 0.981 & 0.978 & 0.991 \\
\hline & & 4 & 1.026 & 1.002 & 1.021 & 0.997 & 1.022 & 1.034 \\
\hline & & 1 & 1.052 & 1.042 & 1.050 & 1.066 & 1.054 & 1.034 \\
\hline & & $1 / 4$ & 1.062 & 1.162 & 1.185 & 1.381 & 1.106 & 1.187 \\
\hline & & $1 / 19$ & 1.190 & 1.251 & 1.237 & 1.238 & 1.218 & 1.102 \\
\hline & & $1 / 99$ & 1.241 & 1.153 & 1.173 & 1.071 & 1.073 & 1.151 \\
\hline \multirow{6}{*}{100} & \multirow{6}{*}{10} & 19 & 0.973 & 0.977 & 0.974 & 0.987 & 0.987 & 0.981 \\
\hline & & 4 & 0.993 & 0.985 & 0.992 & 0.991 & 0.988 & 0.985 \\
\hline & & 1 & 1.017 & 0.999 & 1.011 & 1.009 & 1.020 & 1.008 \\
\hline & & $1 / 4$ & 1.036 & 1.041 & 1.014 & 1.038 & 1.031 & 1.035 \\
\hline & & $1 / 19$ & 1.031 & 1.071 & 1.026 & 1.167 & 1.083 & 1.024 \\
\hline & & $1 / 99$ & 1.036 & 1.066 & 1.076 & 1.024 & 1.106 & 1.027 \\
\hline \multirow{6}{*}{100} & \multirow{6}{*}{30} & 19 & 0.985 & 0.985 & 0.980 & 0.981 & 0.983 & 0.985 \\
\hline & & 4 & 0.997 & 1.002 & 0.993 & 1.009 & 1.003 & 1.006 \\
\hline & & 1 & 1.033 & 1.055 & 1.037 & 1.015 & 1.044 & 1.046 \\
\hline & & $1 / 4$ & 1.023 & 1.034 & 1.027 & 1.029 & 1.084 & 1.037 \\
\hline & & $1 / 19$ & 1.068 & 1.135 & 1.048 & 1.131 & 1.105 & 1.095 \\
\hline & & $1 / 99$ & 1.064 & 1.025 & 1.038 & 1.112 & 1.100 & 1.075 \\
\hline \multirow{6}{*}{100} & \multirow{6}{*}{50} & 19 & 0.991 & 0.988 & 0.984 & 0.986 & 0.986 & 0.988 \\
\hline & & 4 & 1.009 & 1.010 & 1.004 & 1.017 & 1.006 & 1.012 \\
\hline & & 1 & 1.046 & 1.023 & 1.021 & 1.035 & 1.030 & 1.020 \\
\hline & & $1 / 4$ & 1.046 & 1.116 & 1.048 & 1.029 & 1.086 & 1.062 \\
\hline & & $1 / 19$ & 1.068 & 1.175 & 1.110 & 1.149 & 1.124 & 1.069 \\
\hline & & $1 / 99$ & 1.054 & 1.112 & 1.018 & 1.053 & 1.034 & 1.189 \\
\hline \multirow{6}{*}{300} & \multirow{6}{*}{30} & 19 & 0.978 & 0.985 & 0.980 & 0.981 & 0.986 & 0.970 \\
\hline & & 4 & 0.997 & 0.985 & 0.987 & 0.992 & 0.996 & 0.992 \\
\hline & & 1 & 1.008 & 1.000 & 0.998 & 1.000 & 1.006 & 1.017 \\
\hline & & $1 / 4$ & 1.008 & 1.004 & 1.008 & 1.004 & 1.019 & 1.005 \\
\hline & & $1 / 19$ & 1.002 & 1.004 & 1.041 & 1.007 & 1.003 & 1.048 \\
\hline & & $1 / 99$ & 1.011 & 1.135 & 1.002 & 1.018 & 1.023 & 1.008 \\
\hline \multirow{6}{*}{300} & \multirow{6}{*}{50} & 19 & 0.986 & 0.982 & 0.986 & 0.986 & 0.991 & 0.986 \\
\hline & & 4 & 0.997 & 0.992 & 0.990 & 0.994 & 0.990 & 0.992 \\
\hline & & 1 & 0.998 & 1.016 & 1.003 & 1.013 & 1.001 & 1.006 \\
\hline & & $1 / 4$ & 1.011 & 0.998 & 0.998 & 1.007 & 1.005 & 1.004 \\
\hline & & $1 / 19$ & 1.005 & 1.031 & 1.045 & 1.117 & 1.097 & 1.006 \\
\hline & & $1 / 99$ & 1.028 & 1.037 & 1.019 & 1.054 & 1.015 & 1.034 \\
\hline \multirow{6}{*}{300} & & 19 & 0.995 & 0.993 & 0.989 & 0.993 & 0.988 & 0.993 \\
\hline & & 4 & 0.998 & 0.995 & 0.993 & 0.999 & 0.992 & 0.994 \\
\hline & 100 & 1 & 1.008 & 0.995 & 0.992 & 0.995 & 0.993 & 1.000 \\
\hline & & $1 / 4$ & 1.002 & 0.999 & 1.000 & 1.000 & 1.008 & 1.003 \\
\hline & & $1 / 19$ & 1.006 & 1.015 & 1.001 & 1.001 & 1.004 & 1.002 \\
\hline & & $1 / 99$ & 1.003 & 1.007 & 1.002 & 1.015 & 1.032 & 1.039 \\
\hline
\end{tabular}


Apêndice E

Quocientes EQM $\left[\hat{Q}_{i}^{(1)}\right] / \operatorname{EMQ}\left[\hat{Q}_{i}^{(2)}\right]$ sob erros de medida endógenos com distribuição normal 
Figura E.1: Quocientes $\operatorname{EQM}\left[\hat{Q}_{i}^{(1)}\right] / \operatorname{EMQ}\left[\hat{Q}_{i}^{(2)}\right]$ sob erros de medida endógenos com distribuição normal, $N=50$ e $n=5$.

Y's gerados com distribuição uniforme
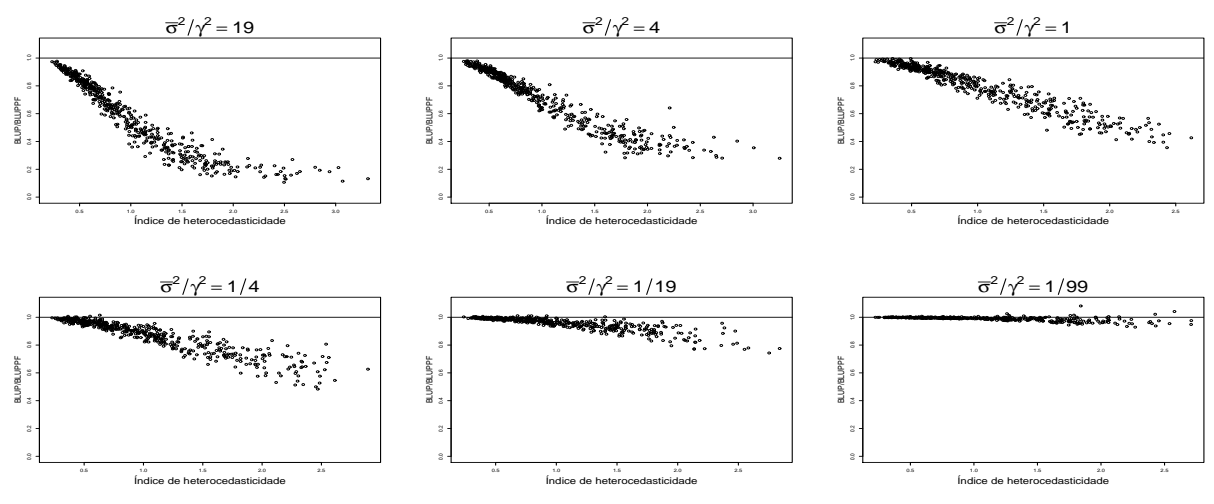

Y's gerados com distribuição assimétrica à esquerda
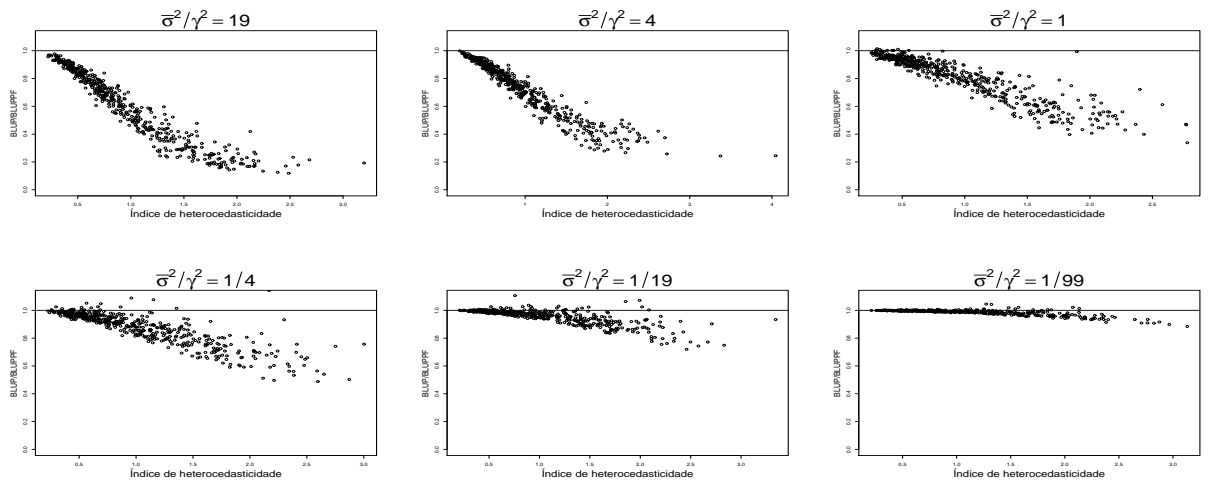

Y's gerados com distribuição assimétrica à direita
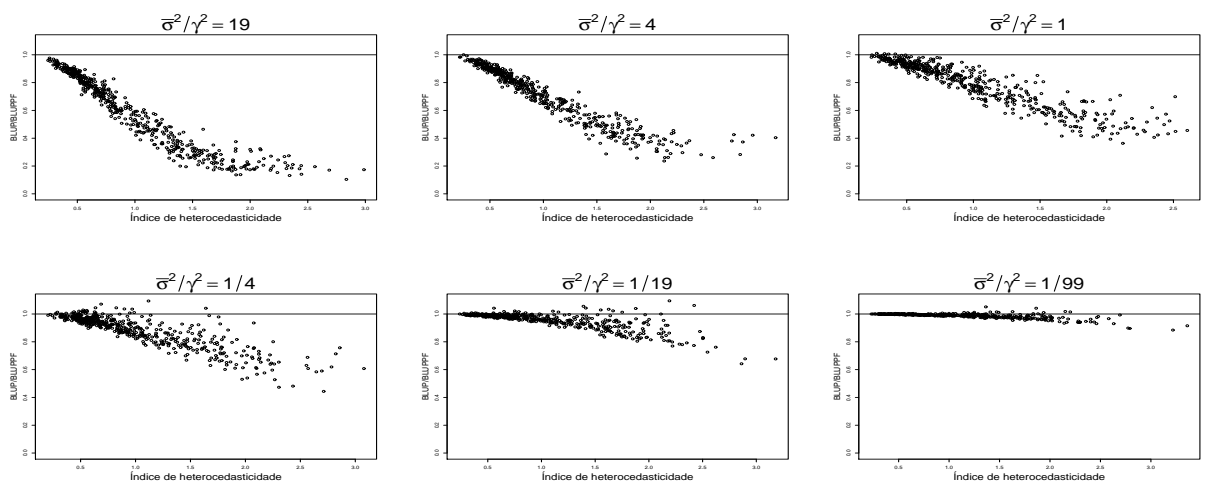
Figura E.2: Quocientes $\operatorname{EQM}\left[\hat{Q}_{i}^{(1)}\right] / \operatorname{EMQ}\left[\hat{Q}_{i}^{(2)}\right]$ sob erros de medida endógenos com distribuição normal, $N=50$ e $n=10$.

Y's gerados com distribuição uniforme
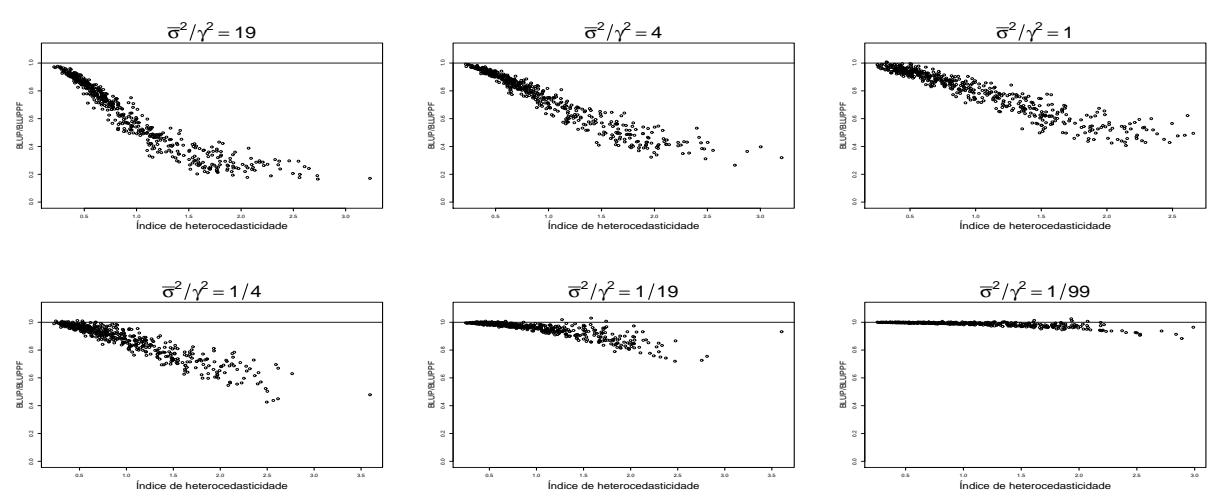

Y's gerados com distribuição assimétrica à esquerda
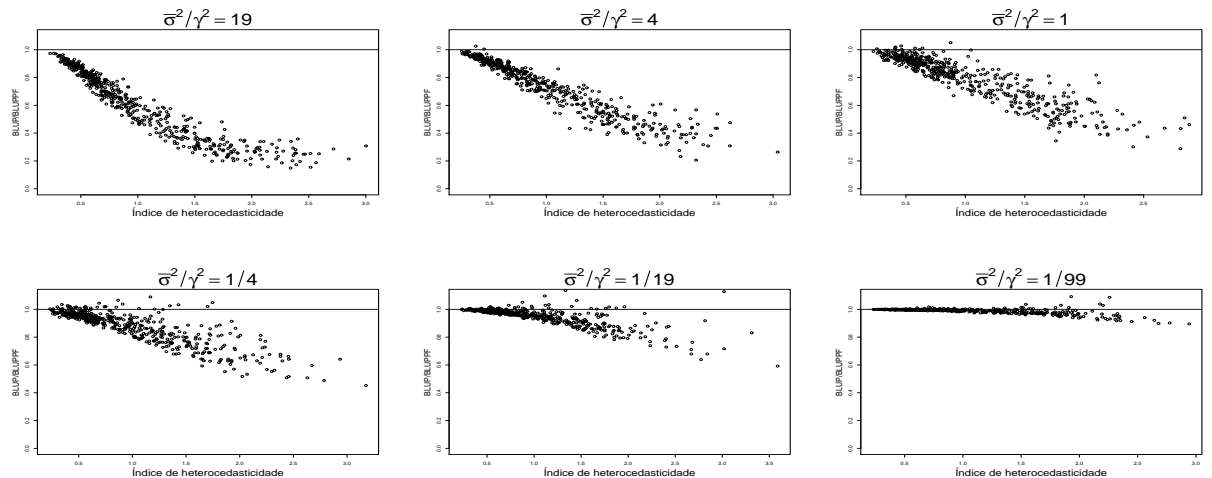

Y's gerados com distribuição assimétrica à direita
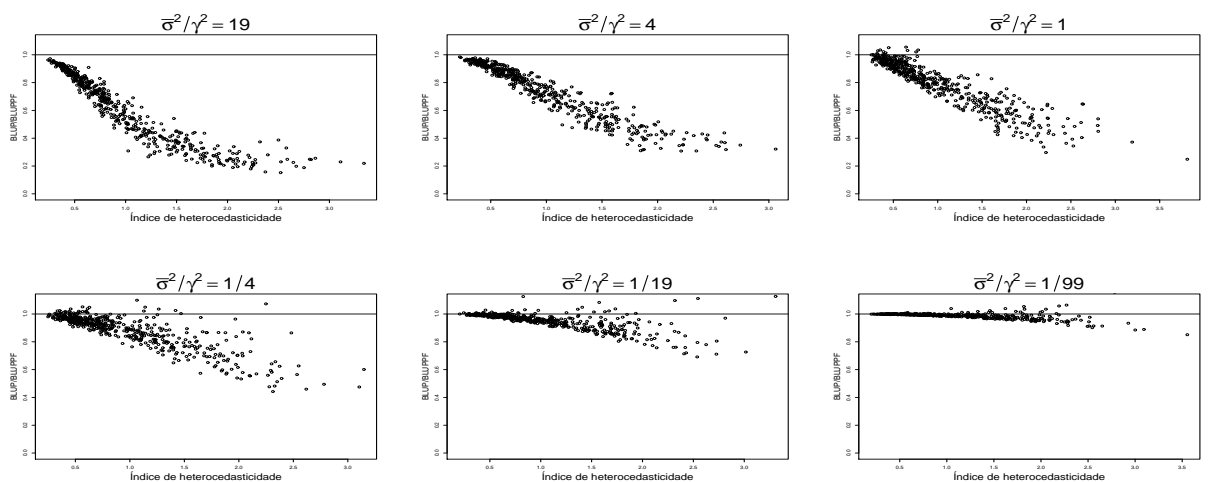
Figura E.3: Quocientes EQM $\left[\hat{Q}_{i}^{(1)}\right] / \operatorname{EMQ}\left[\hat{Q}_{i}^{(2)}\right]$ sob erros de medida endógenos com distribuição normal, $N=50$ e $n=25$.

Y's gerados com distribuição uniforme
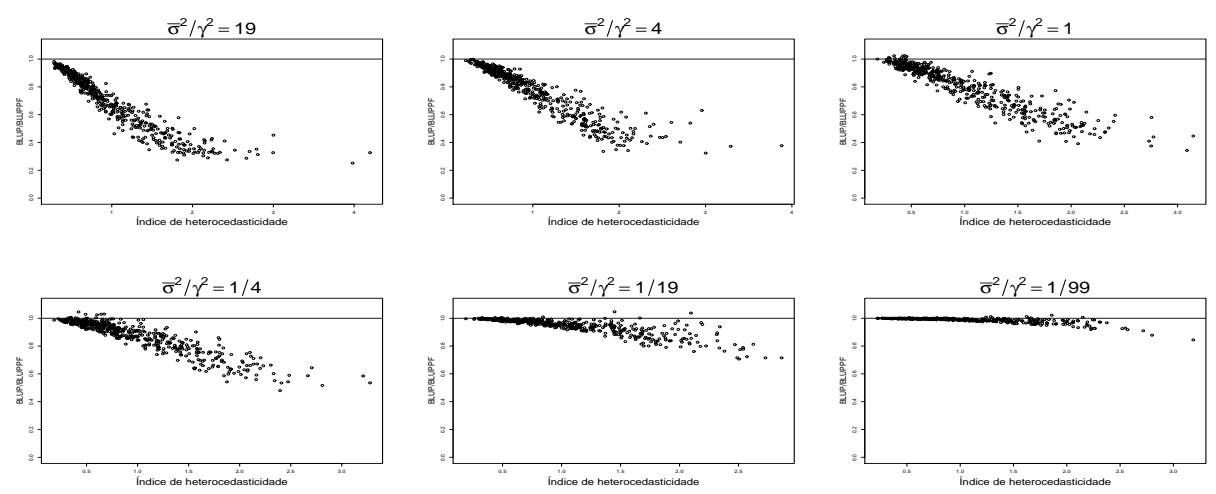

Y's gerados com distribuição assimétrica à esquerda
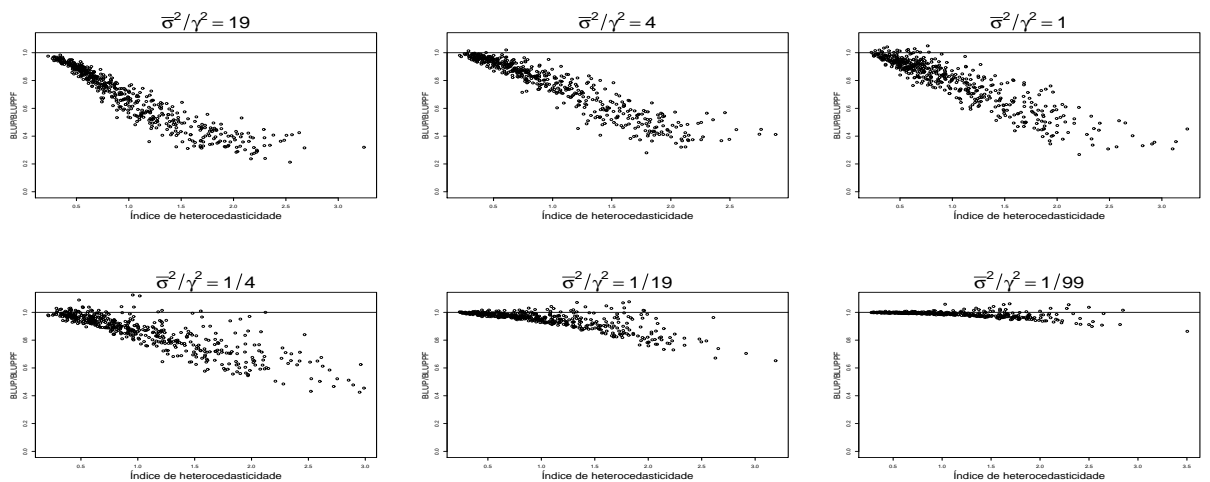

Y's gerados com distribuição assimétrica à direita
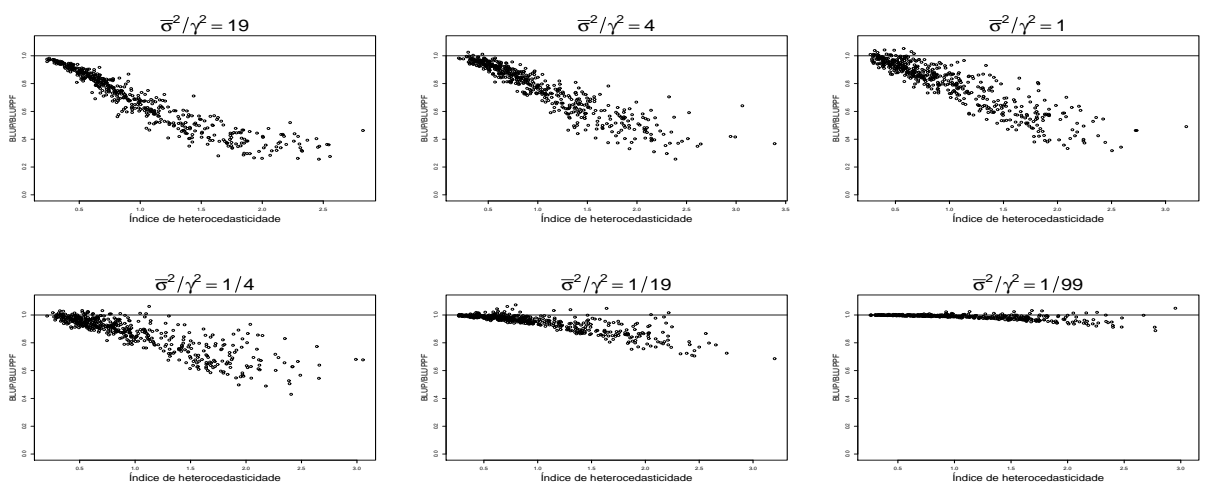
Figura E.4: Quocientes $\operatorname{EQM}\left[\hat{Q}_{i}^{(1)}\right] / \operatorname{EMQ}\left[\hat{Q}_{i}^{(2)}\right]$ sob erros de medida endógenos com distribuição normal, $N=100$ e $n=10$.

Y's gerados com distribuição uniforme
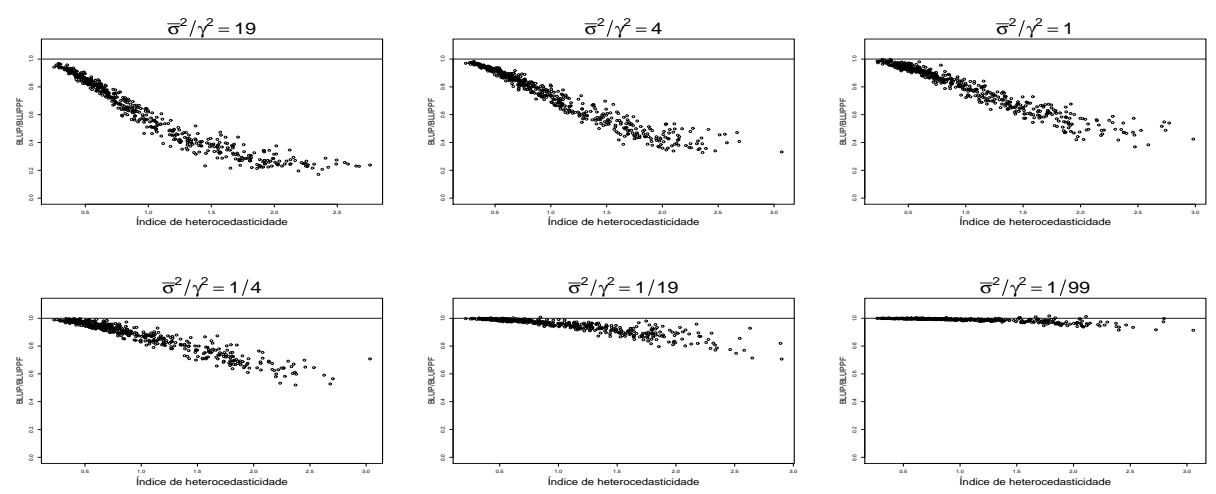

Y's gerados com distribuição assimétrica à esquerda
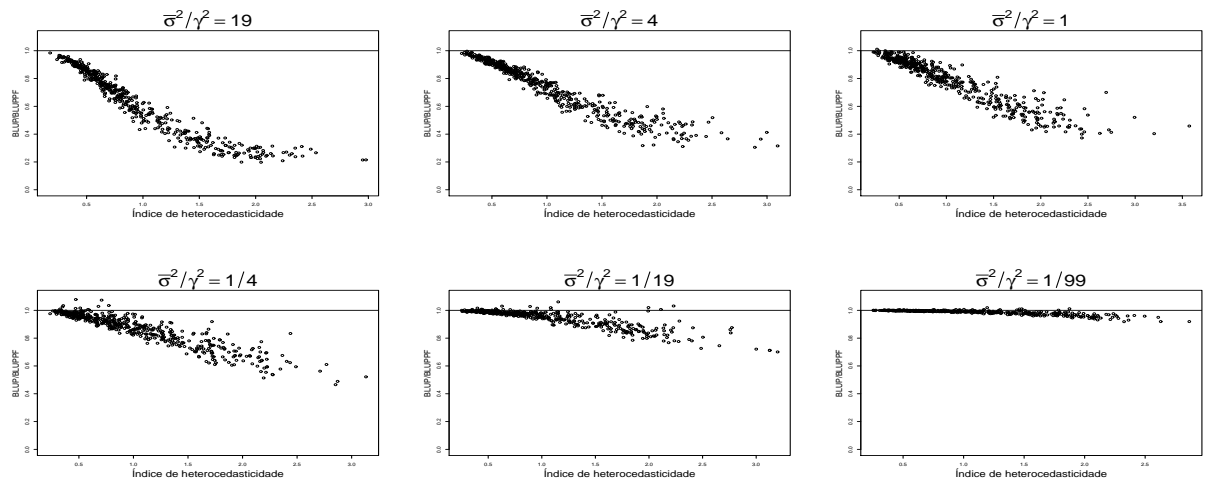

Y's gerados com distribuição assimétrica à direita
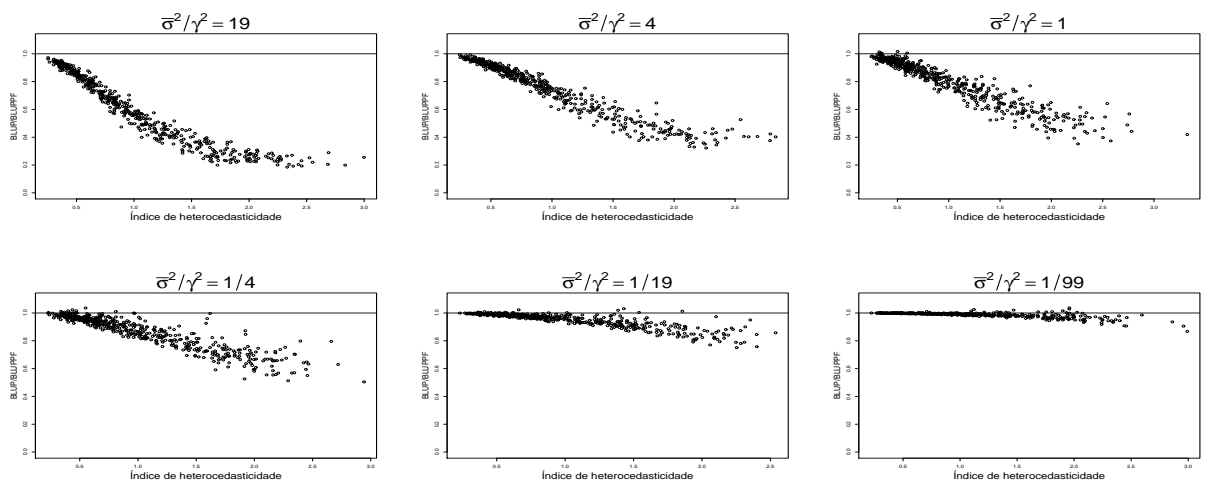
Figura E.5: Quocientes $\operatorname{EQM}\left[\hat{Q}_{i}^{(1)}\right] / \operatorname{EMQ}\left[\hat{Q}_{i}^{(2)}\right]$ sob erros de medida endógenos com distribuição normal, $N=100$ e $n=30$.

Y's gerados com distribuição uniforme
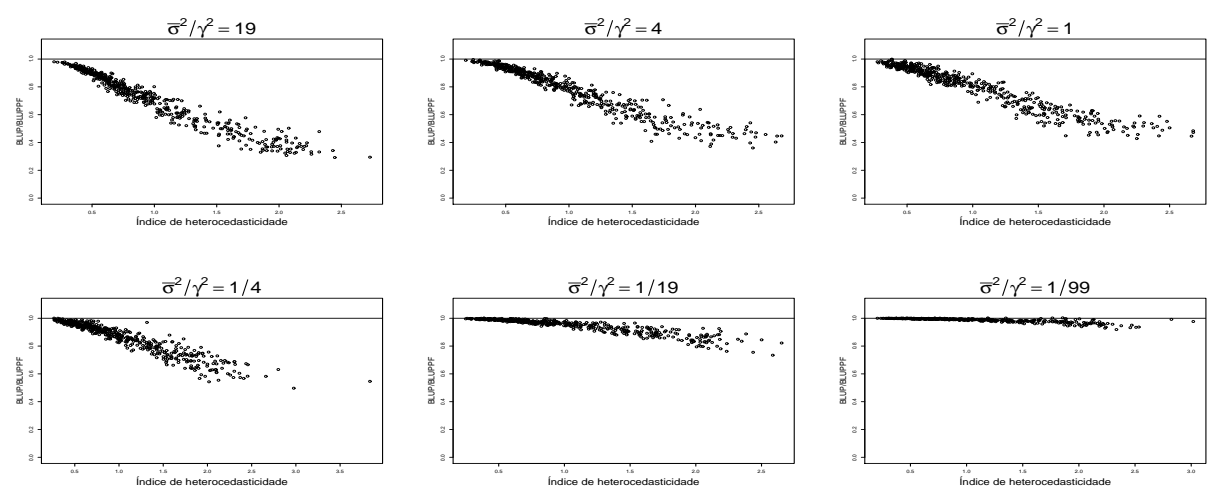

Y's gerados com distribuição assimétrica à esquerda
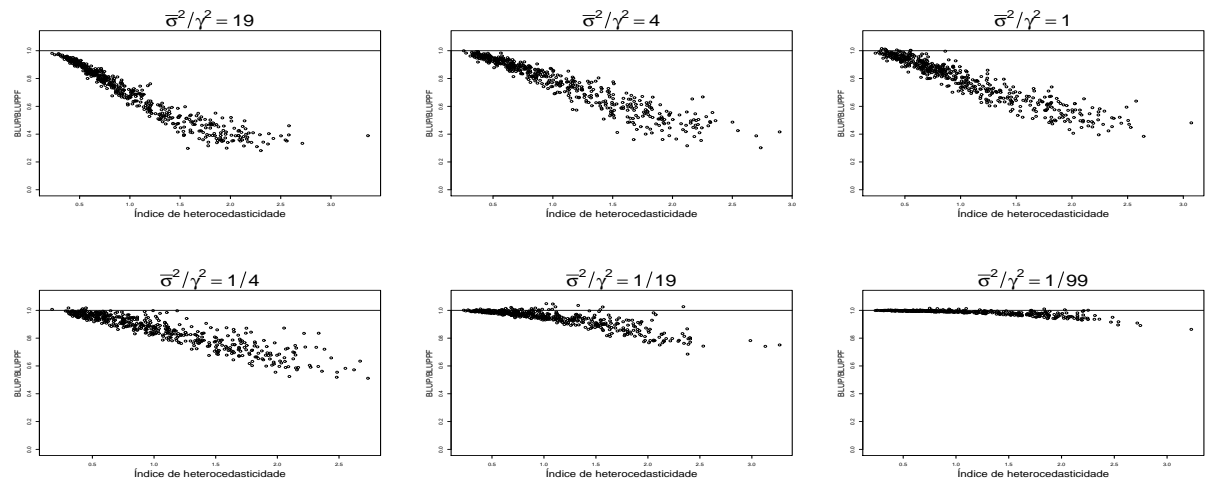

Y's gerados com distribuição assimétrica à direita
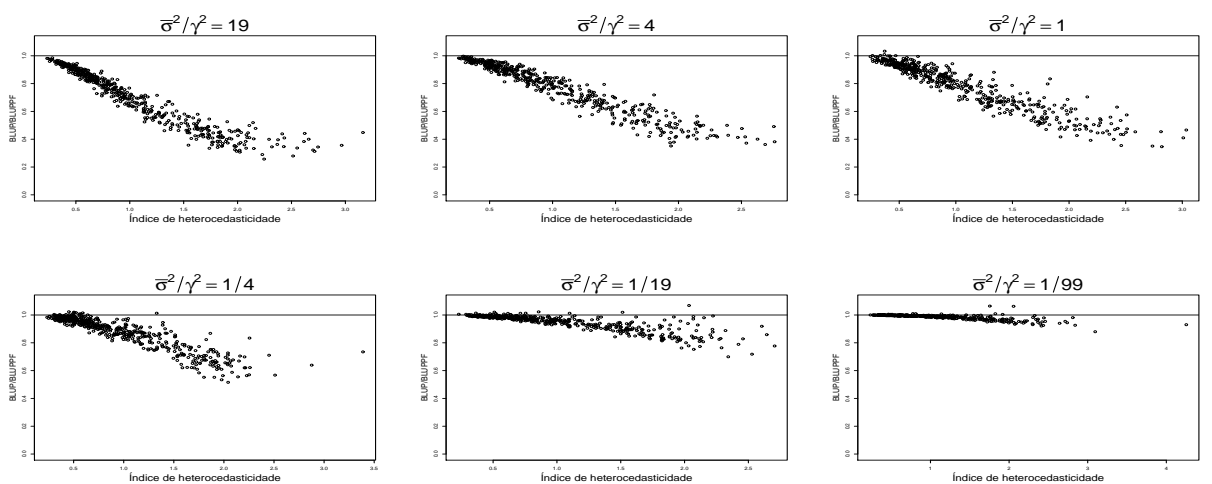
Figura E.6: Quocientes $\operatorname{EQM}\left[\hat{Q}_{i}^{(1)}\right] / \operatorname{EMQ}\left[\hat{Q}_{i}^{(2)}\right]$ sob erros de medida endógenos com distribuição normal, $N=100$ e $n=50$.

Y's gerados com distribuição uniforme
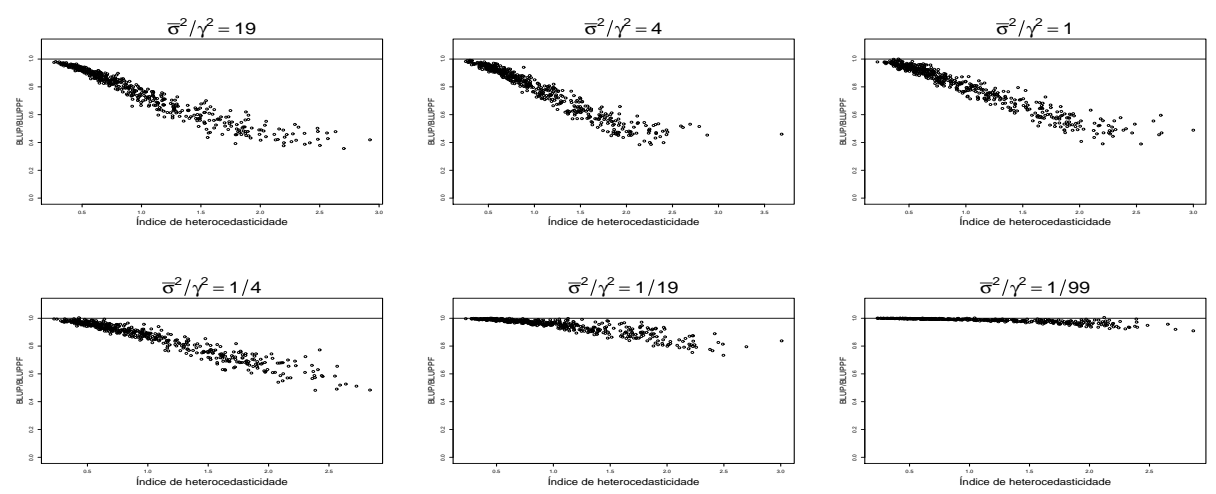

Y's gerados com distribuição assimétrica à esquerda
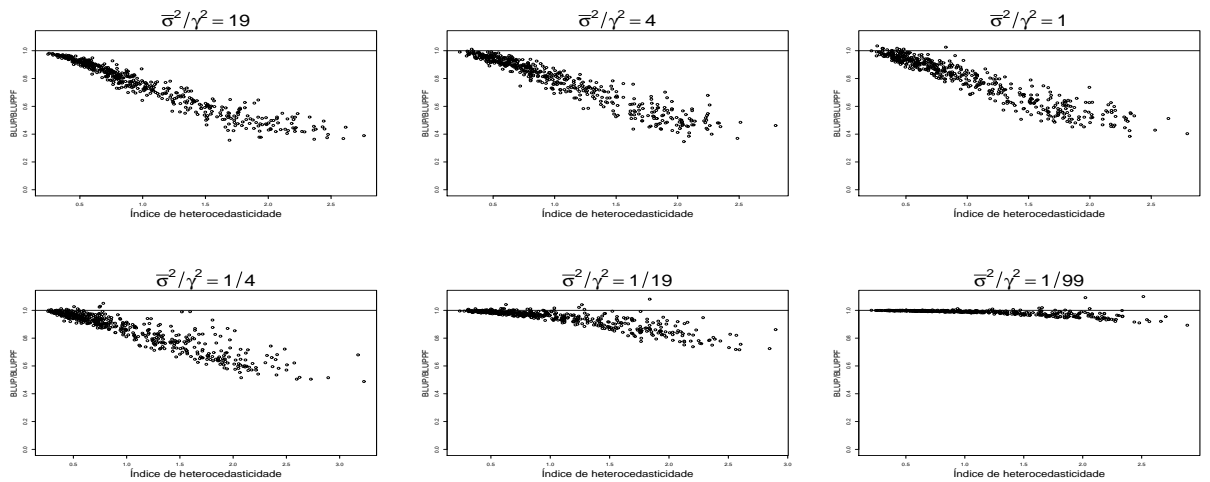

Y's gerados com distribuição assimétrica à direita
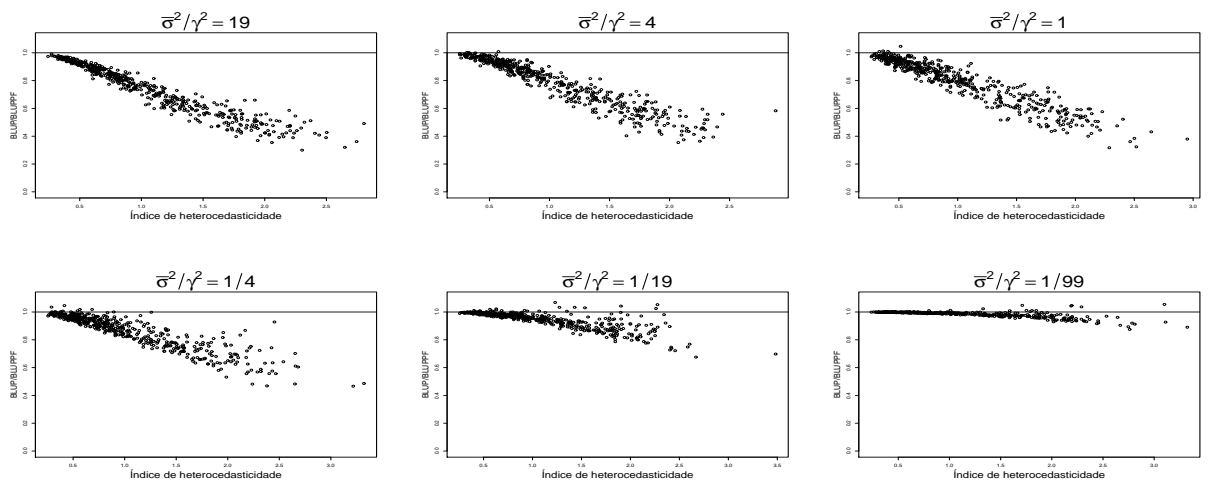
Figura E.7: Quocientes $\operatorname{EQM}\left[\hat{Q}_{i}^{(1)}\right] / \operatorname{EMQ}\left[\hat{Q}_{i}^{(2)}\right]$ sob erros de medida endógenos com distribuição normal, $N=300$ e $n=30$.

Y's gerados com distribuição uniforme
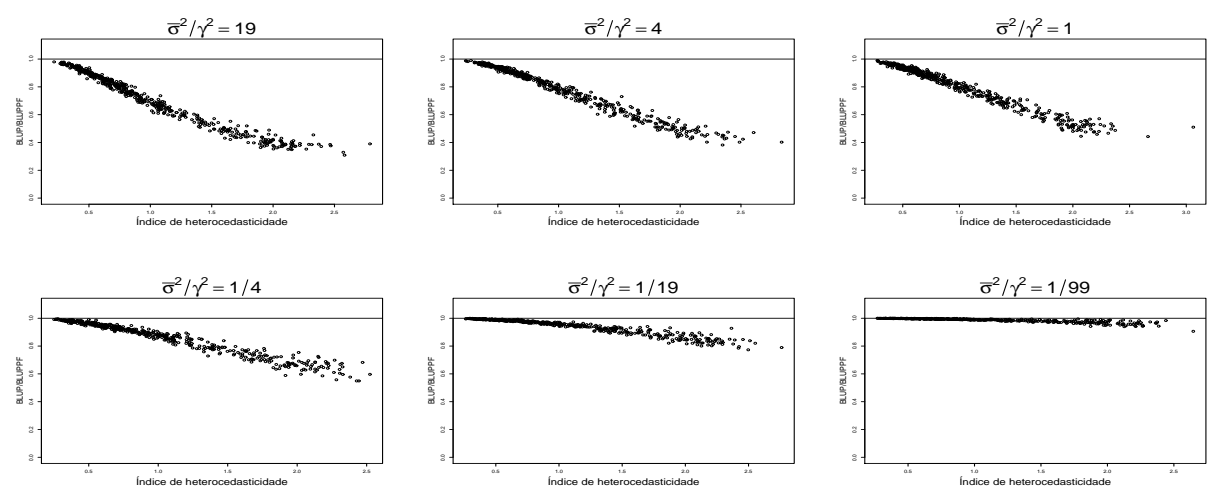

Y's gerados com distribuição assimétrica à esquerda
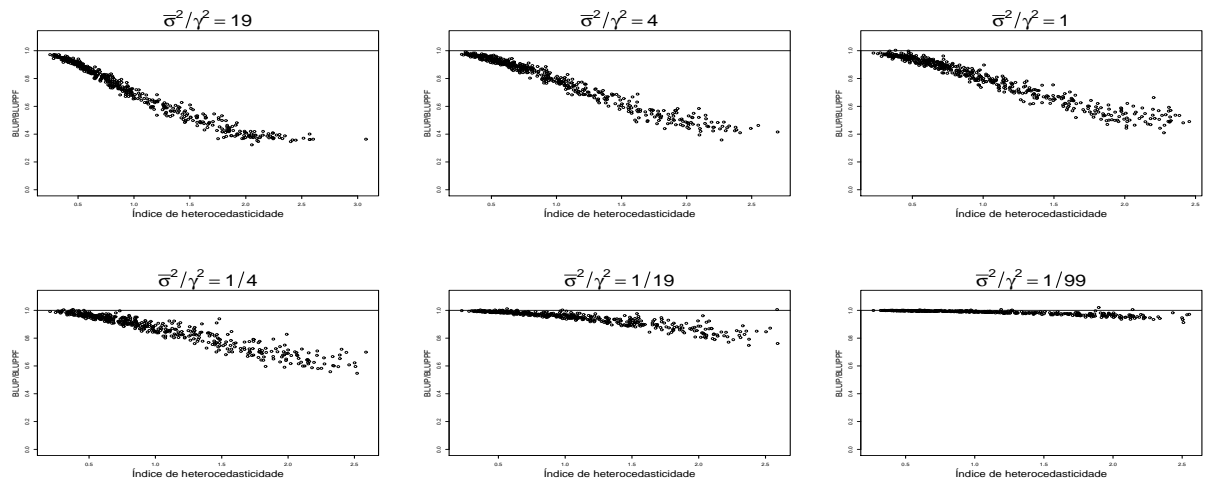

Y's gerados com distribuição assimétrica à direita
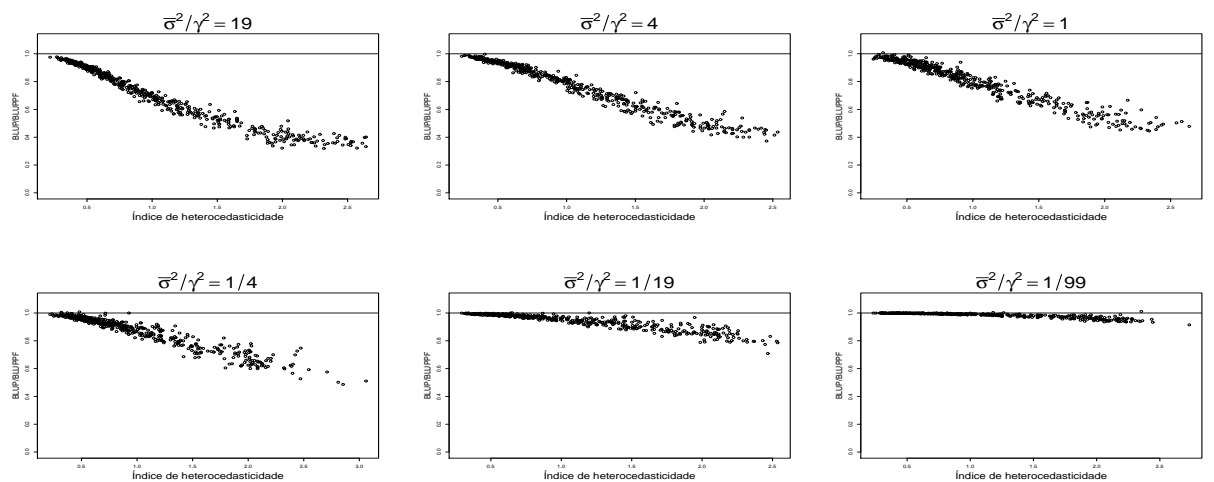
Figura E.8: Quocientes $\operatorname{EQM}\left[\hat{Q}_{i}^{(1)}\right] / \operatorname{EMQ}\left[\hat{Q}_{i}^{(2)}\right]$ sob erros de medida endógenos com distribuição normal, $N=300$ e $n=50$.

Y's gerados com distribuição uniforme
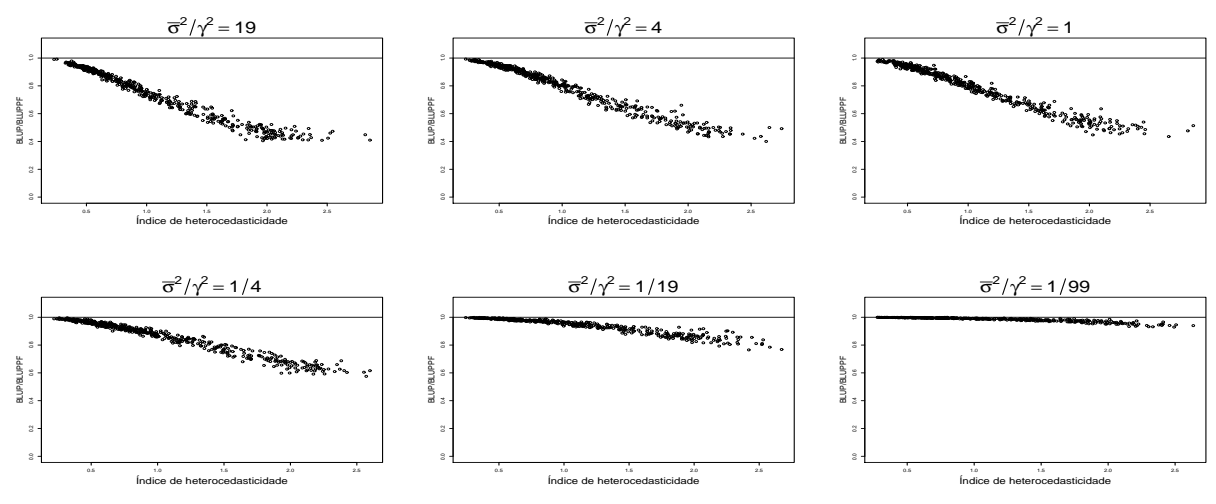

Y's gerados com distribuição assimétrica à esquerda
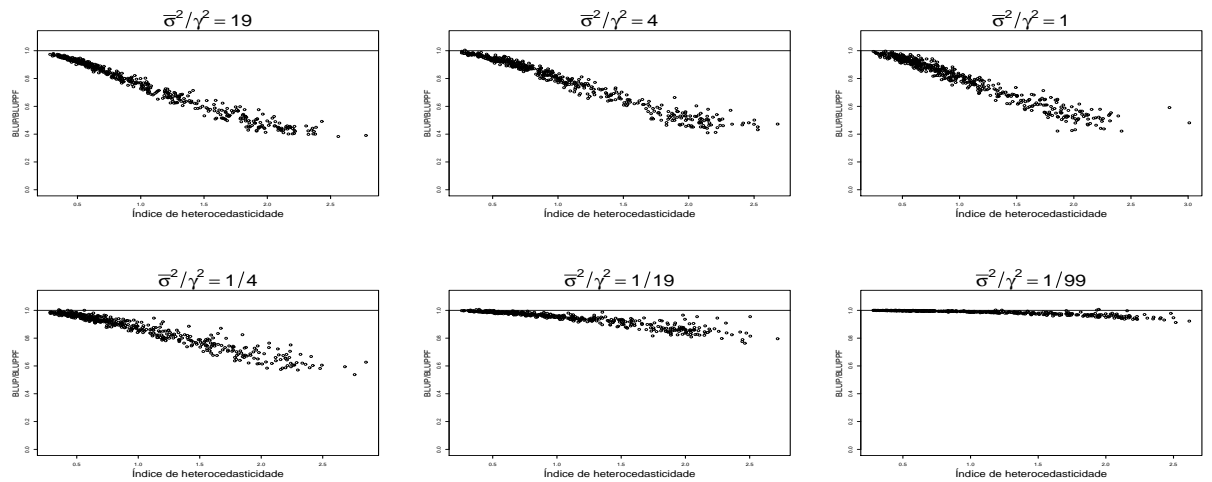

Y's gerados com distribuição assimétrica à direita
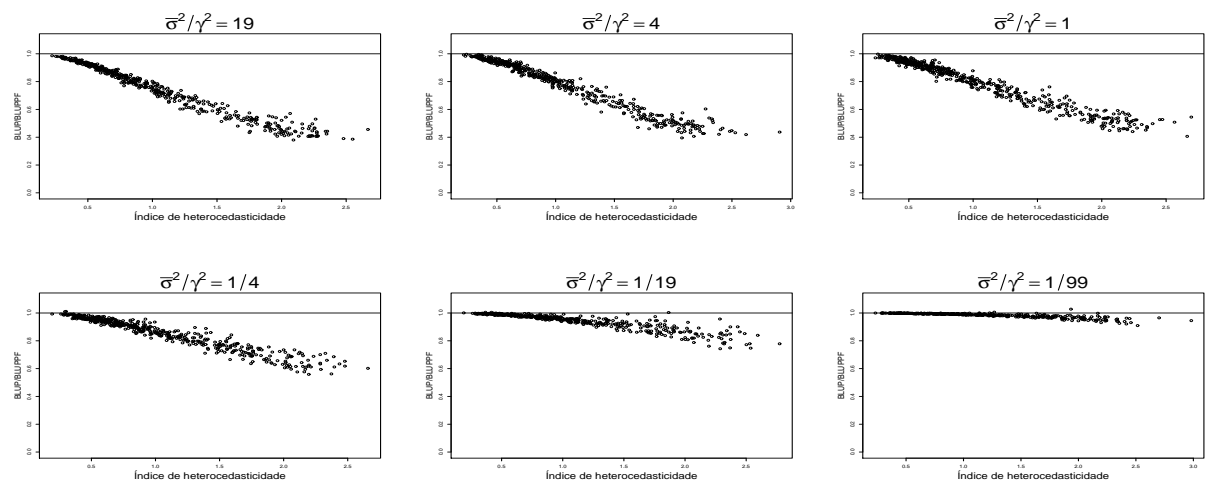
Figura E.9: Quocientes $\operatorname{EQM}\left[\hat{Q}_{i}^{(1)}\right] / \operatorname{EMQ}\left[\hat{Q}_{i}^{(2)}\right]$ sob erros de medida endógenos com distribuição normal, $N=300$ e $n=100$.

Y's gerados com distribuição uniforme
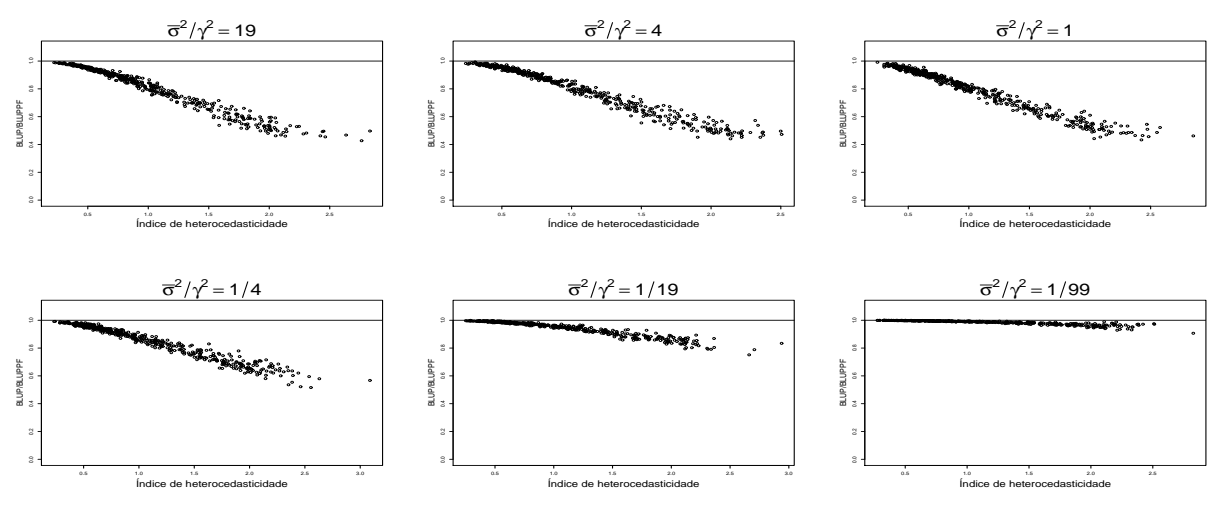

Y's gerados com distribuição assimétrica à esquerda
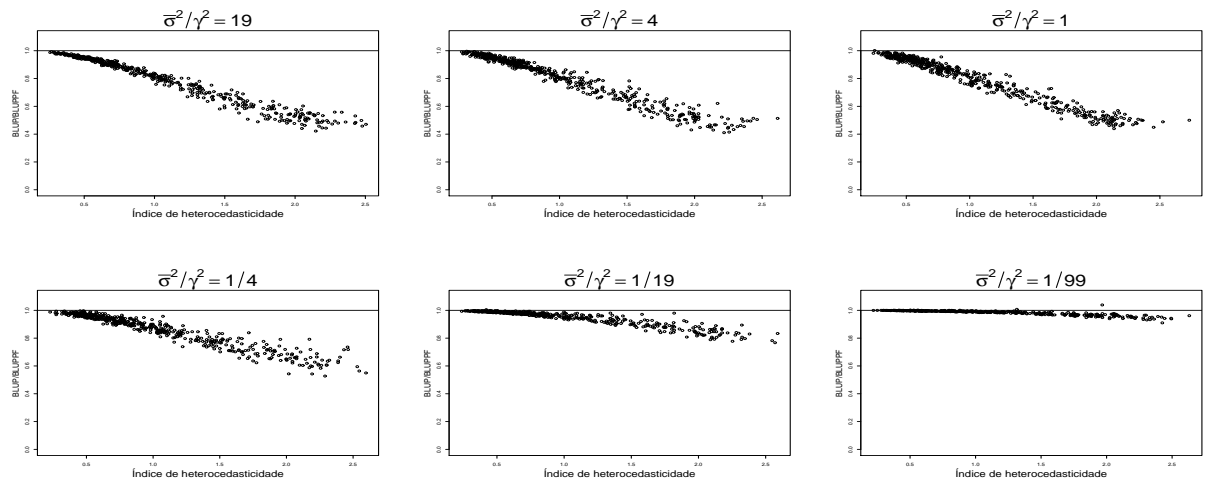

Y's gerados com distribuição assimétrica à direita
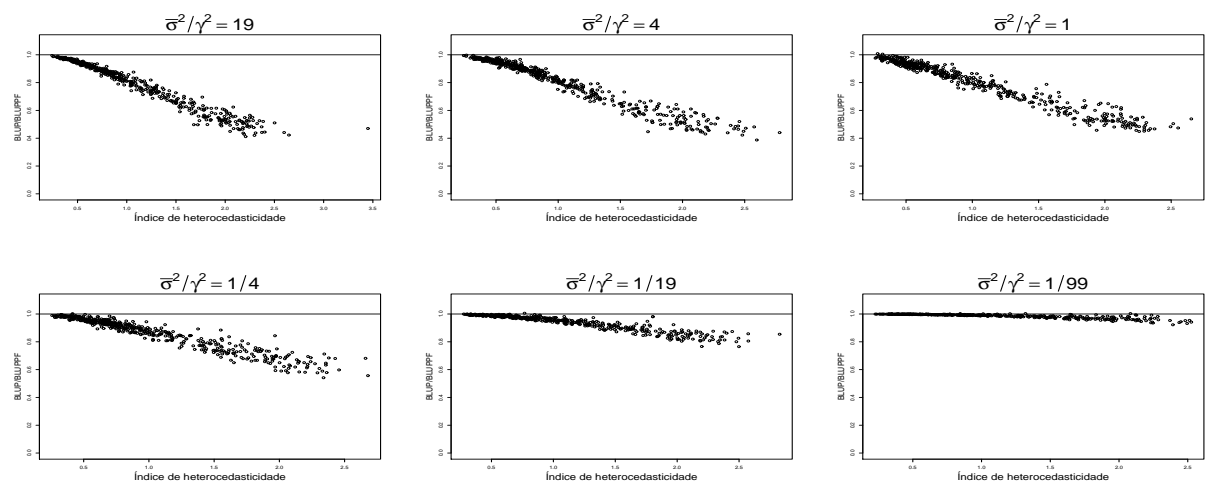
Apêndice $\mathrm{F}$

Quocientes EQM $\left[\hat{Q}_{i}^{(1)}\right] / \operatorname{EMQ}\left[\hat{Q}_{i}^{(2)}\right]$ sob erros de medida endógenos com distribuição uniforme 
Figura F.1: Quocientes $\operatorname{EQM}\left[\hat{Q}_{i}^{(1)}\right] / \operatorname{EMQ}\left[\hat{Q}_{i}^{(2)}\right]$ sob erros de medida endógenos com distribuição uniforme, $N=50$ e $n=5$.

Y's gerados com distribuição uniforme
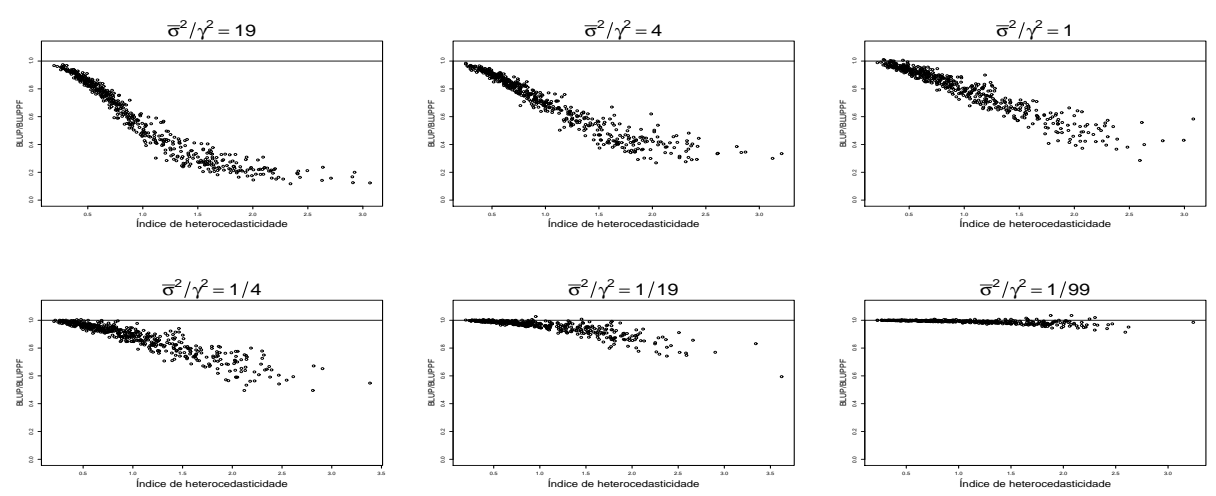

Y's gerados com distribuição assimétrica à esquerda
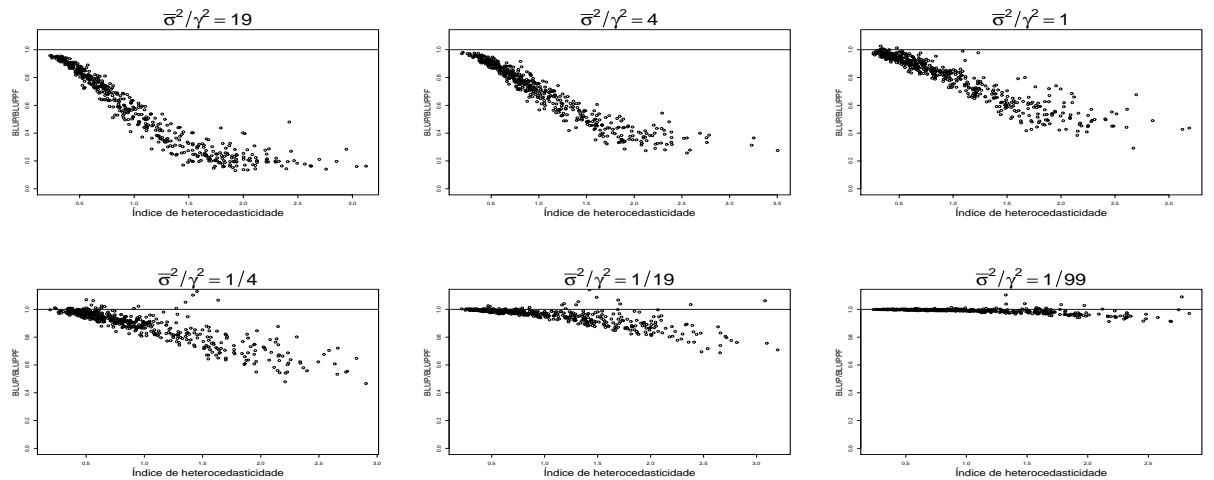

Y's gerados com distribuição assimétrica à direita
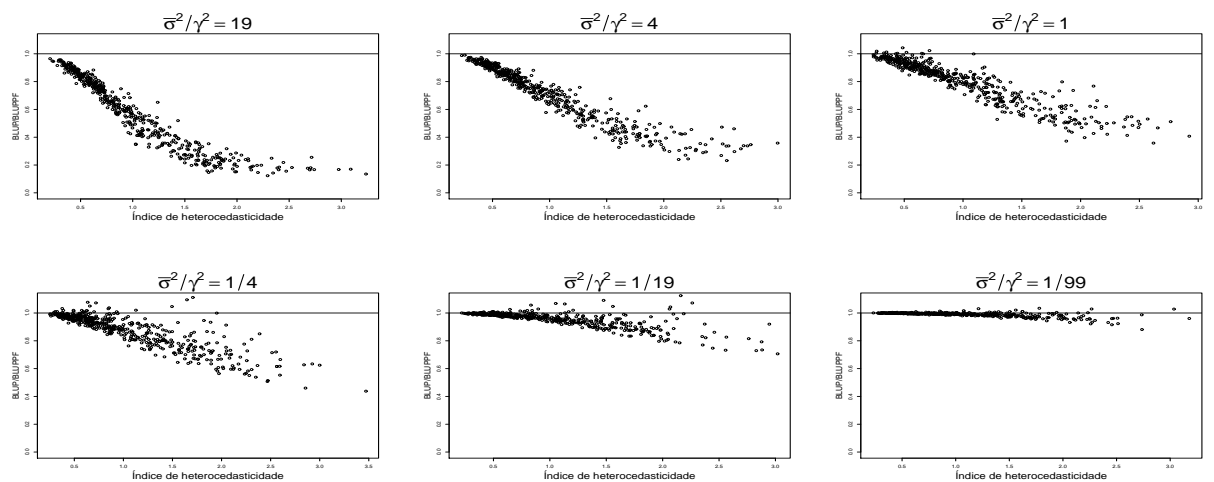
Figura F.2: Quocientes $\operatorname{EQM}\left[\hat{Q}_{i}^{(1)}\right] / \operatorname{EMQ}\left[\hat{Q}_{i}^{(2)}\right]$ sob erros de medida endógenos com distribuição uniforme, $N=50$ e $n=10$.

Y's gerados com distribuição uniforme
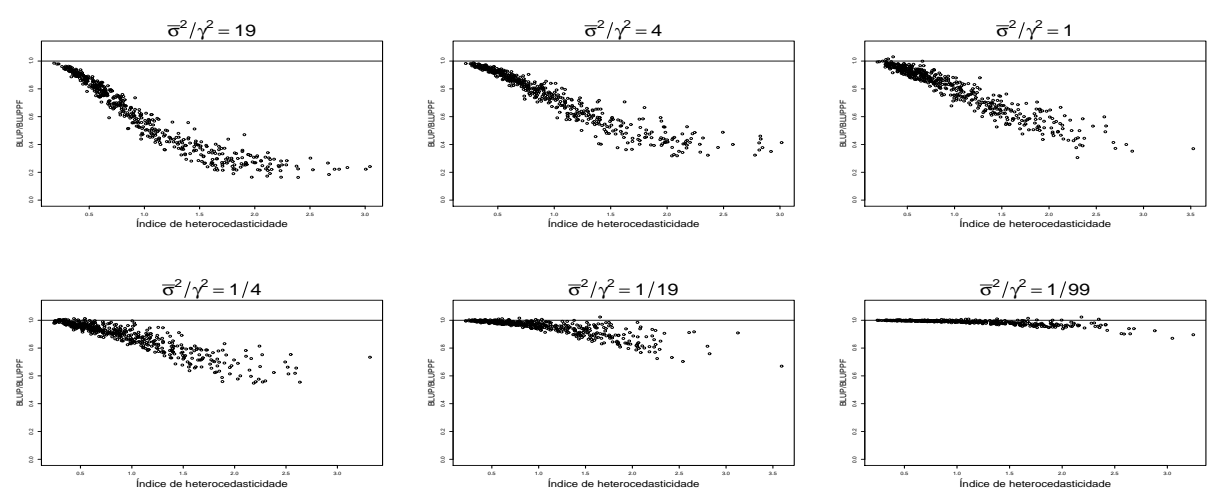

Y's gerados com distribuição assimétrica à esquerda
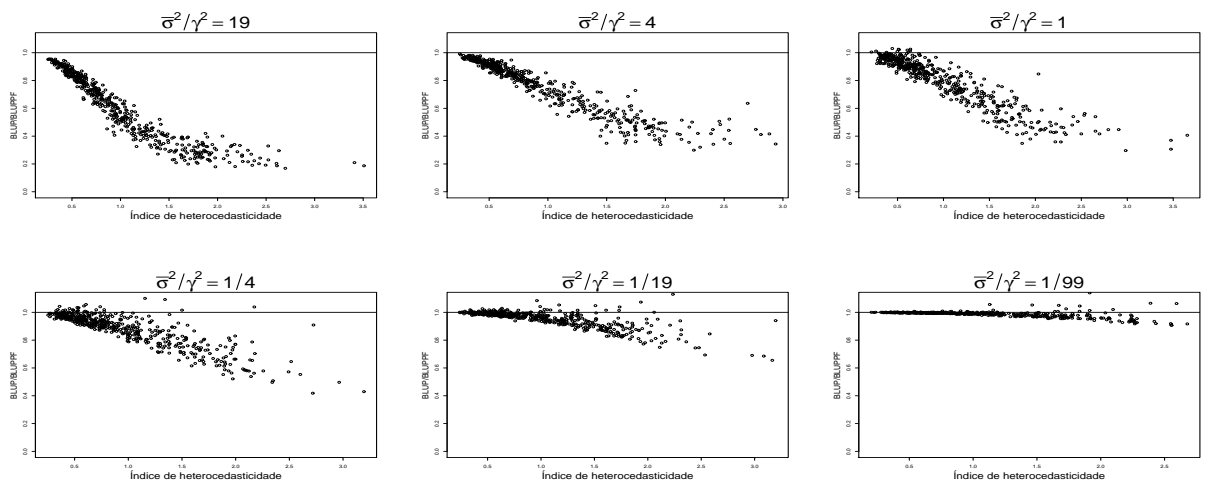

Y's gerados com distribuição assimétrica à direita
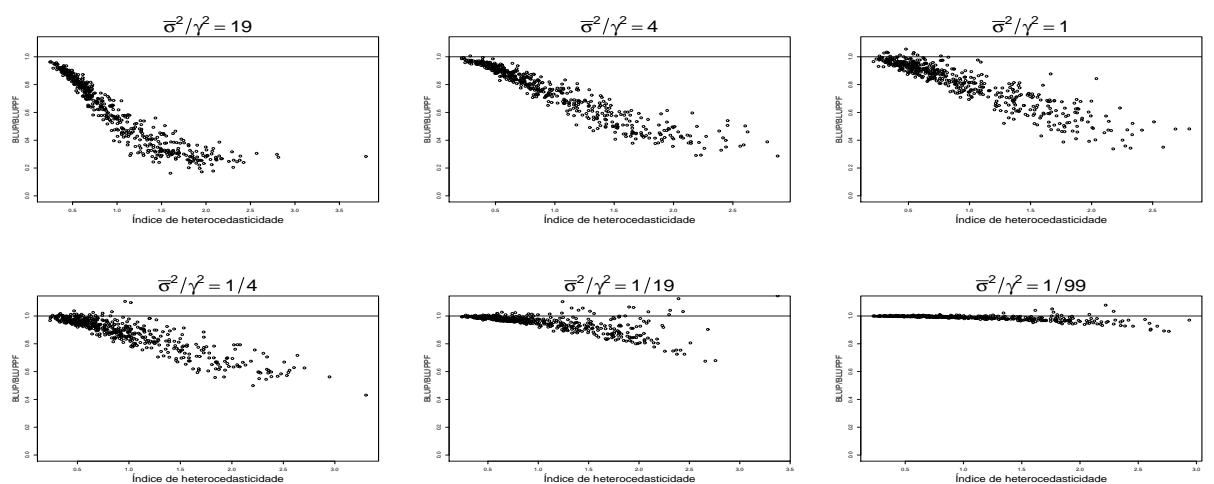
Figura F.3: Quocientes $\operatorname{EQM}\left[\hat{Q}_{i}^{(1)}\right] / \operatorname{EMQ}\left[\hat{Q}_{i}^{(2)}\right]$ sob erros de medida endógenos com distribuição uniforme, $N=50$ e $n=25$.

Y's gerados com distribuição uniforme
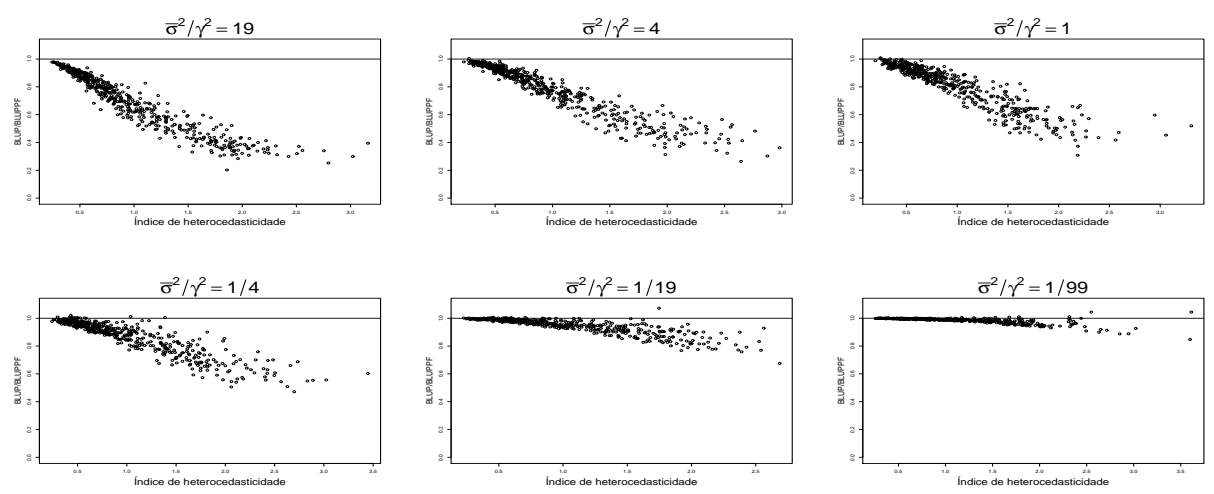

Y's gerados com distribuição assimétrica à esquerda
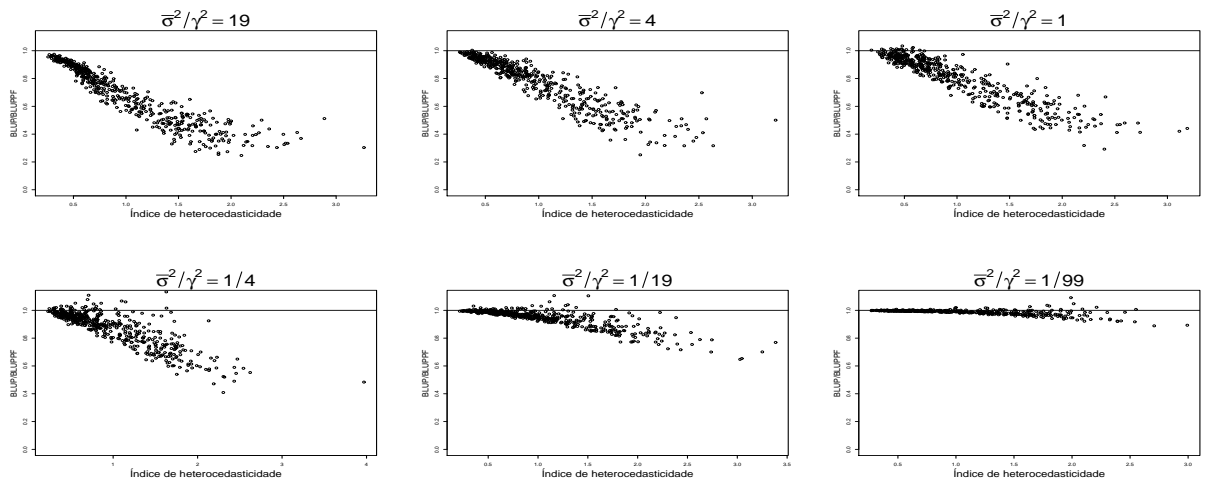

Y's gerados com distribuição assimétrica à direita
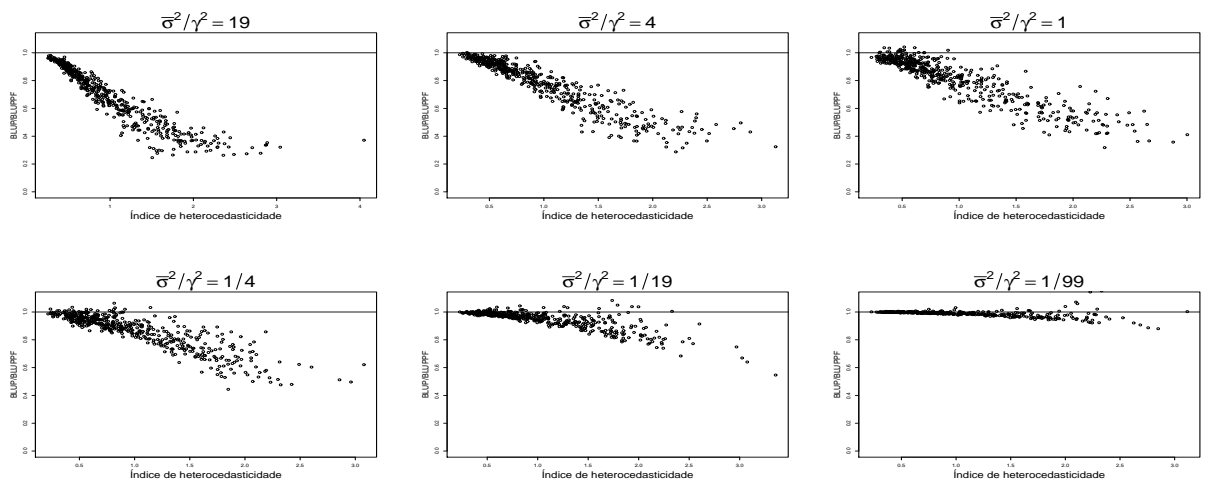
Figura F.4: Quocientes $\operatorname{EQM}\left[\hat{Q}_{i}^{(1)}\right] / \operatorname{EMQ}\left[\hat{Q}_{i}^{(2)}\right]$ sob erros de medida endógenos com distribuição uniforme, $N=100$ e $n=10$.

Y's gerados com distribuição uniforme
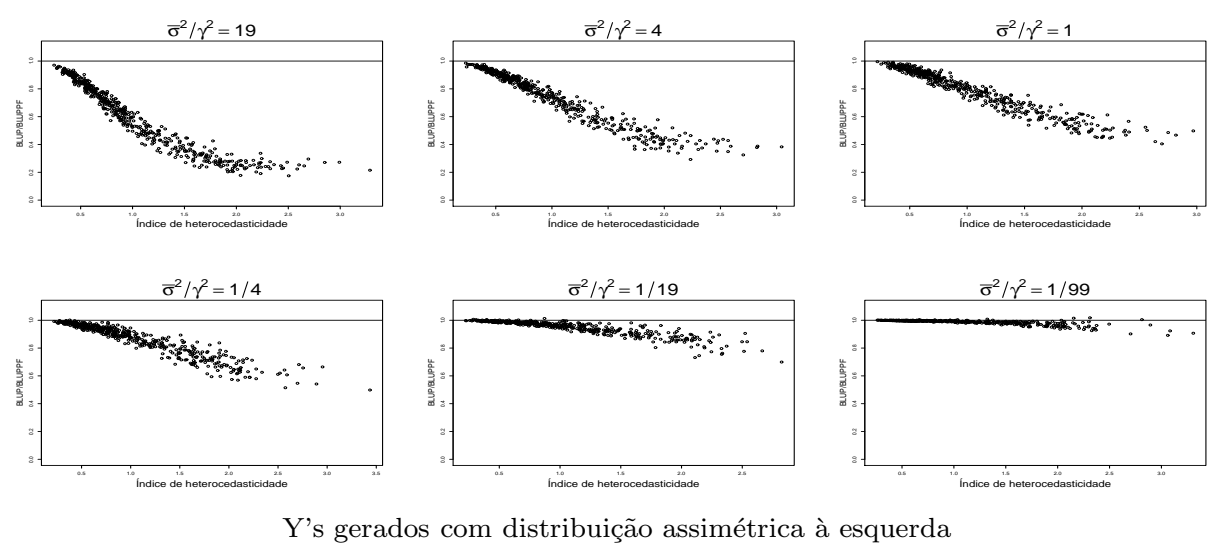

Y's gerados com distribuição assimétrica à esquerda
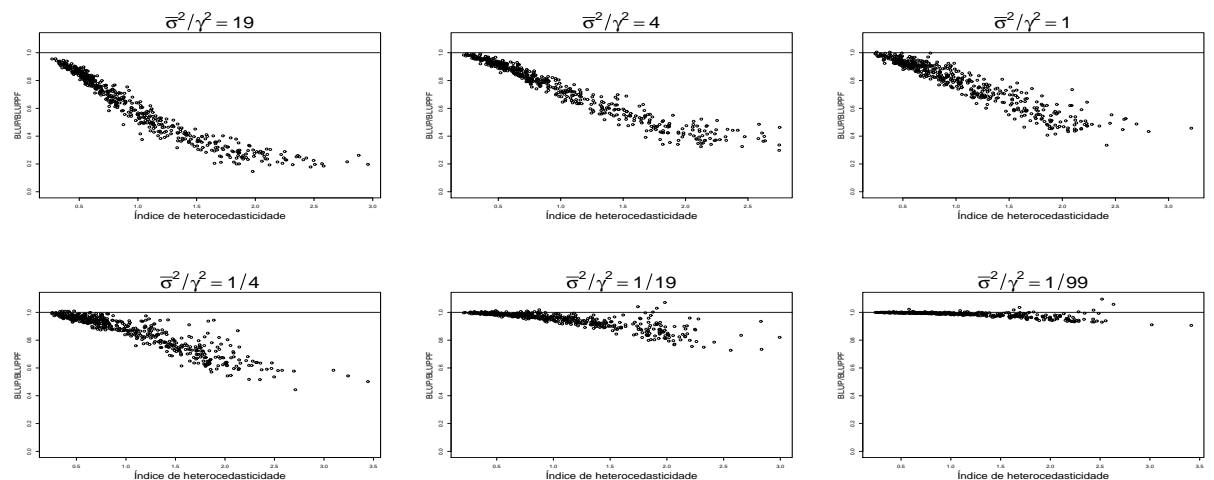

Y's gerados com distribuição assimétrica à direita
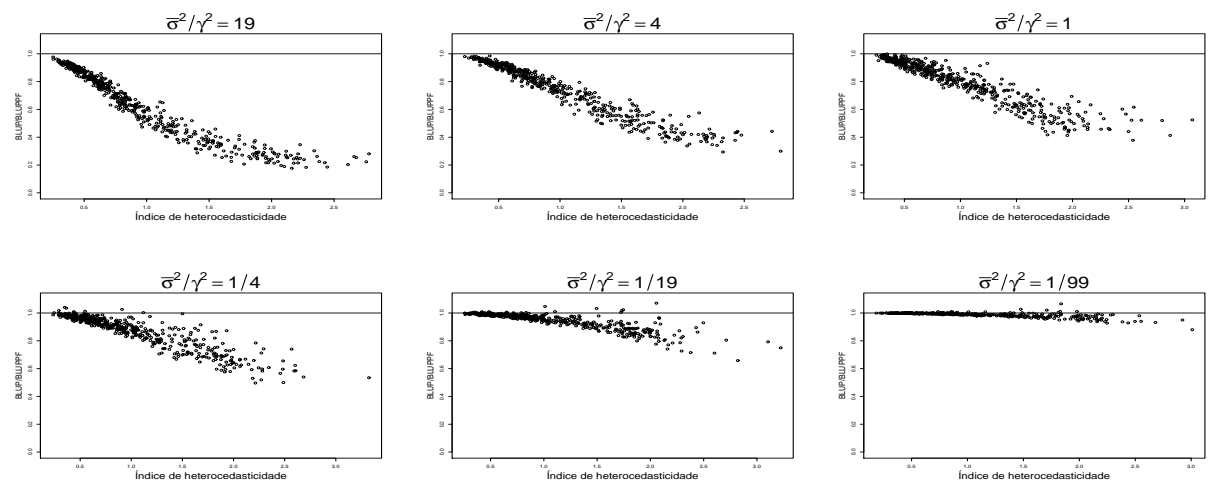
Figura F.5: Quocientes $\operatorname{EQM}\left[\hat{Q}_{i}^{(1)}\right] / \operatorname{EMQ}\left[\hat{Q}_{i}^{(2)}\right]$ sob erros de medida endógenos com distribuição uniforme, $N=100$ e $n=30$.

Y's gerados com distribuição uniforme
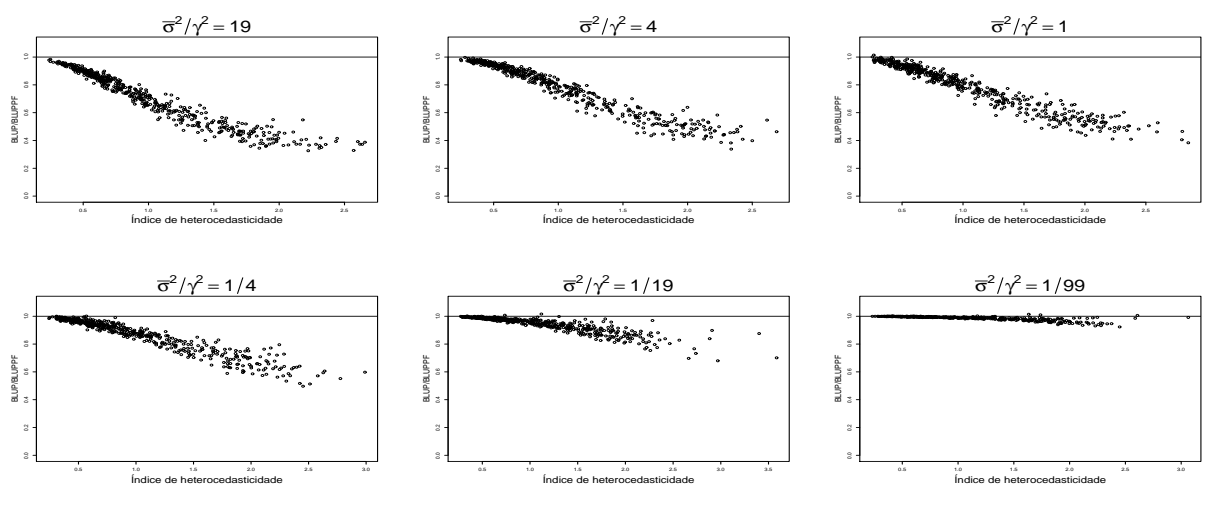

Y's gerados com distribuição assimétrica à esquerda
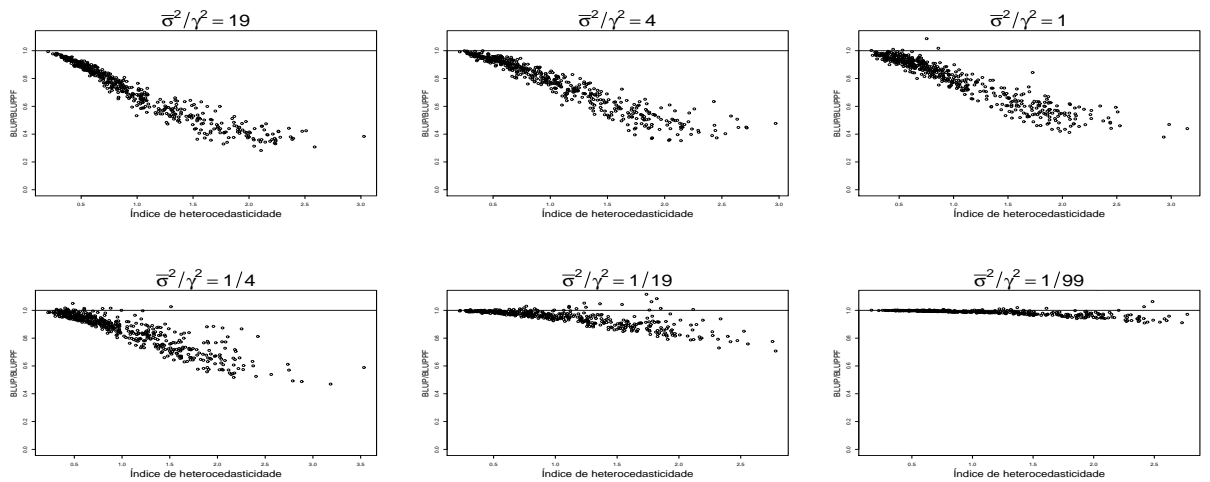

Y's gerados com distribuição assimétrica à direita
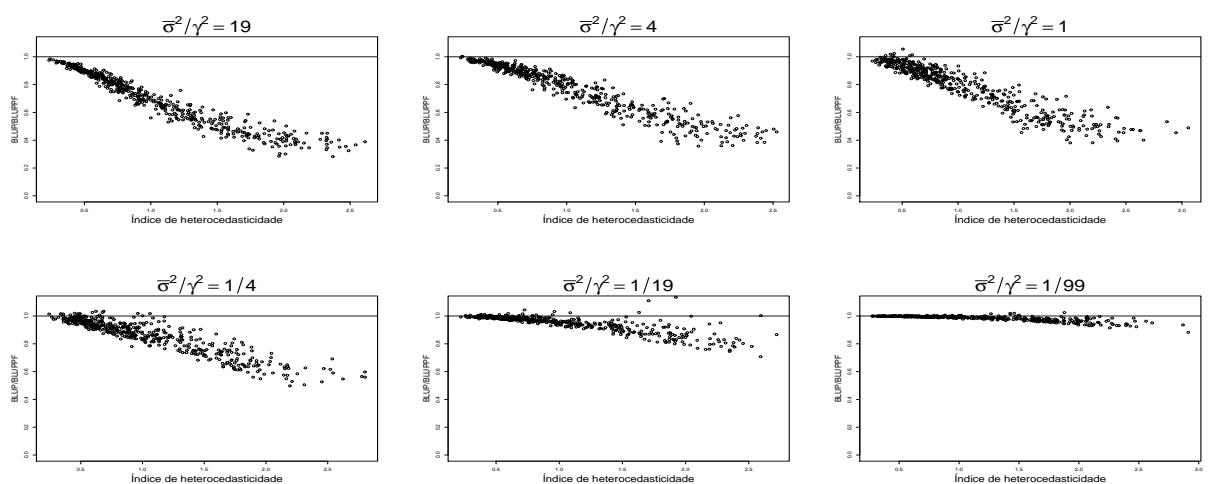
Figura F.6: Quocientes $\operatorname{EQM}\left[\hat{Q}_{i}^{(1)}\right] / \operatorname{EMQ}\left[\hat{Q}_{i}^{(2)}\right]$ sob erros de medida endógenos com distribuição uniforme, $N=100$ e $n=50$.

Y's gerados com distribuição uniforme
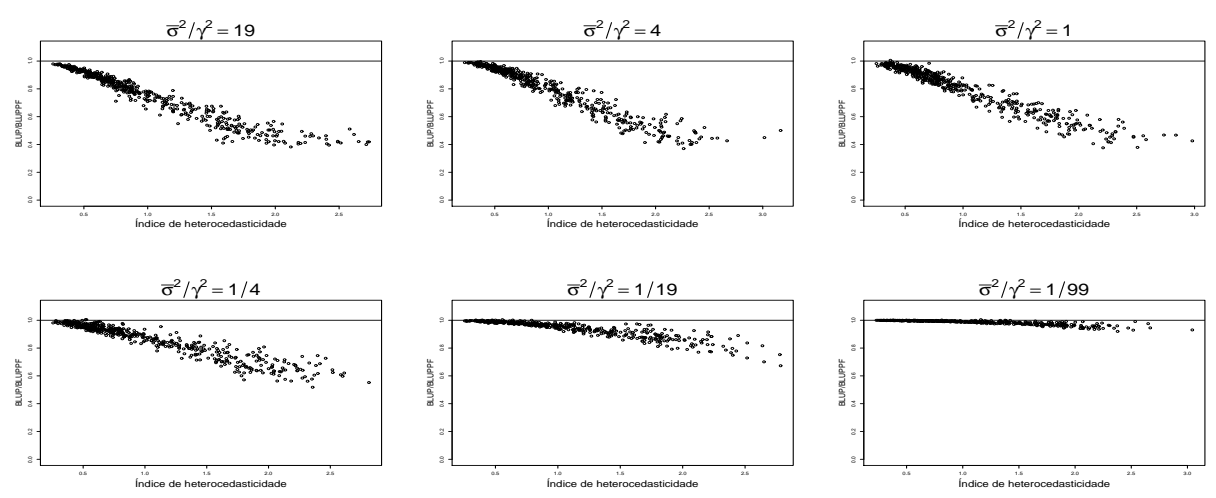

Y's gerados com distribuição assimétrica à esquerda
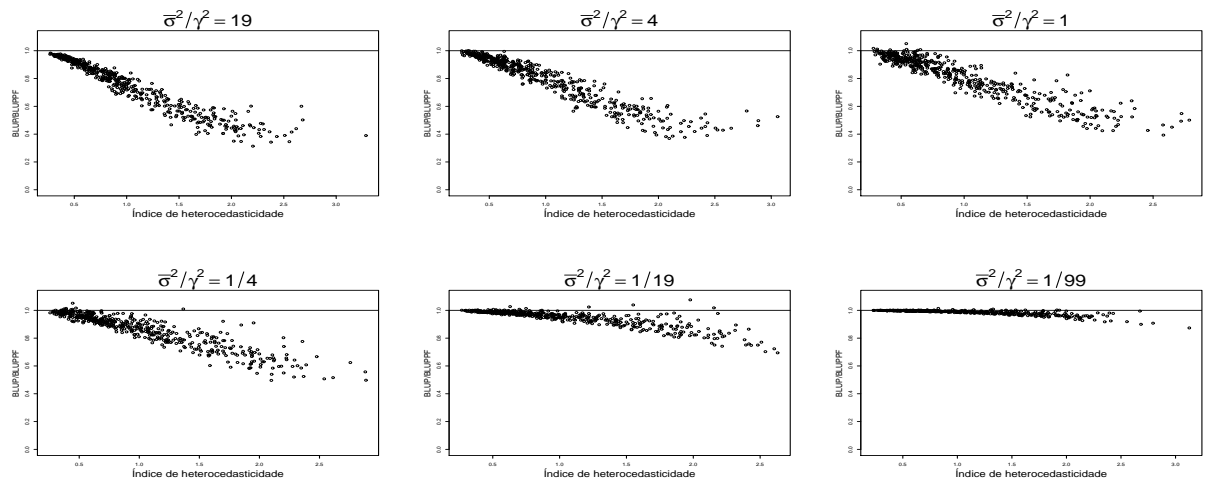

Y's gerados com distribuição assimétrica à direita
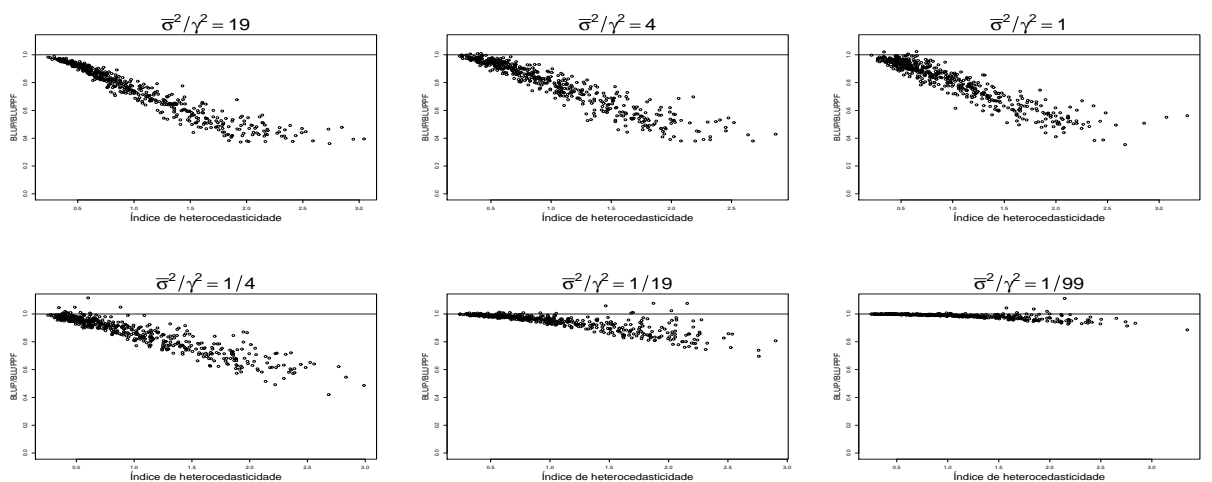
Figura F.7: Quocientes $\operatorname{EQM}\left[\hat{Q}_{i}^{(1)}\right] / \operatorname{EMQ}\left[\hat{Q}_{i}^{(2)}\right]$ sob erros de medida endógenos com distribuição uniforme, $N=300$ e $n=30$.

Y's gerados com distribuição uniforme
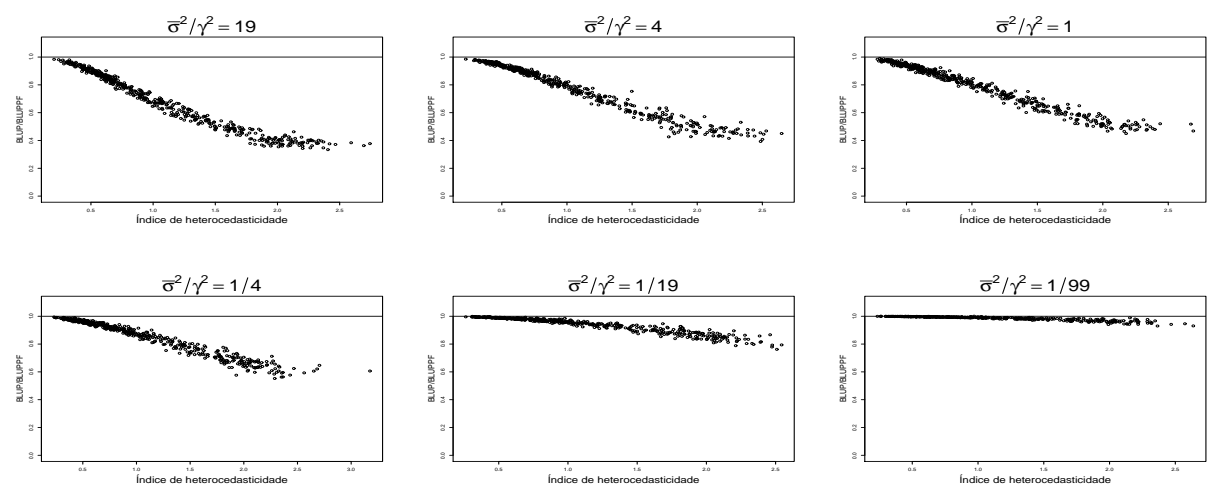

Y's gerados com distribuição assimétrica à esquerda
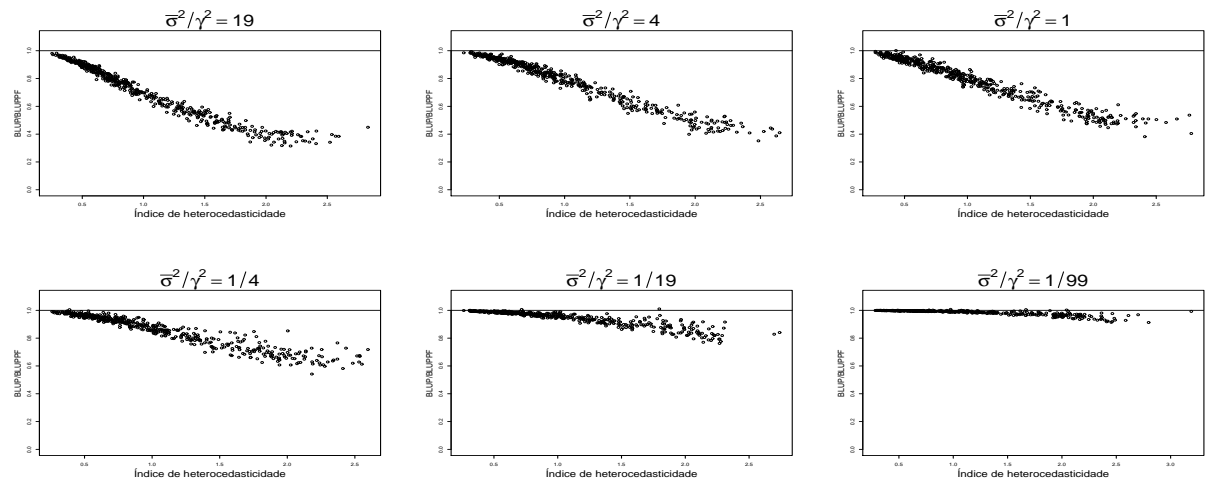

Y's gerados com distribuição assimétrica à direita
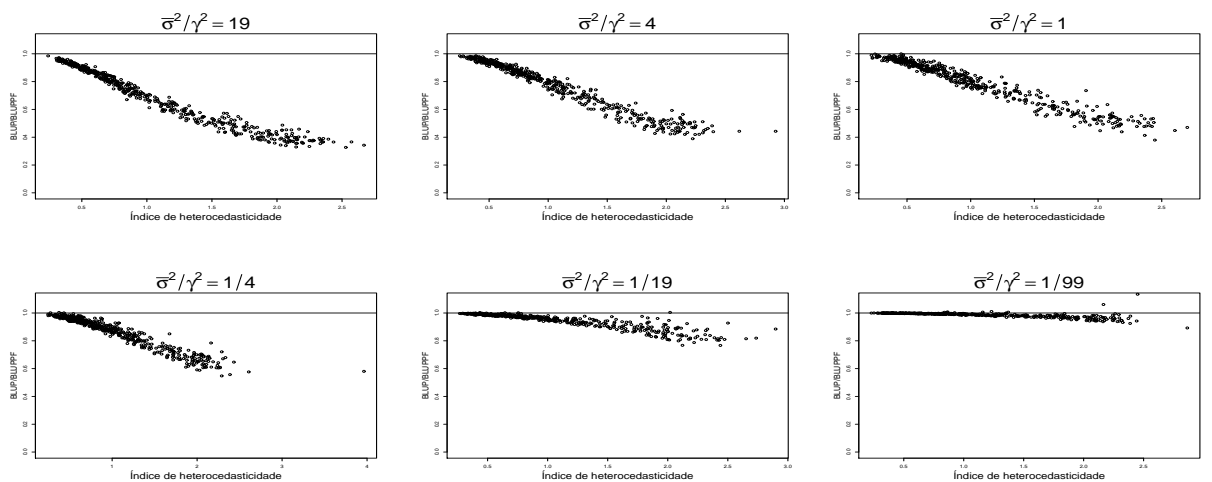
Figura F.8: Quocientes $\operatorname{EQM}\left[\hat{Q}_{i}^{(1)}\right] / \operatorname{EMQ}\left[\hat{Q}_{i}^{(2)}\right]$ sob erros de medida endógenos com distribuição uniforme, $N=300$ e $n=50$.

Y's gerados com distribuição uniforme
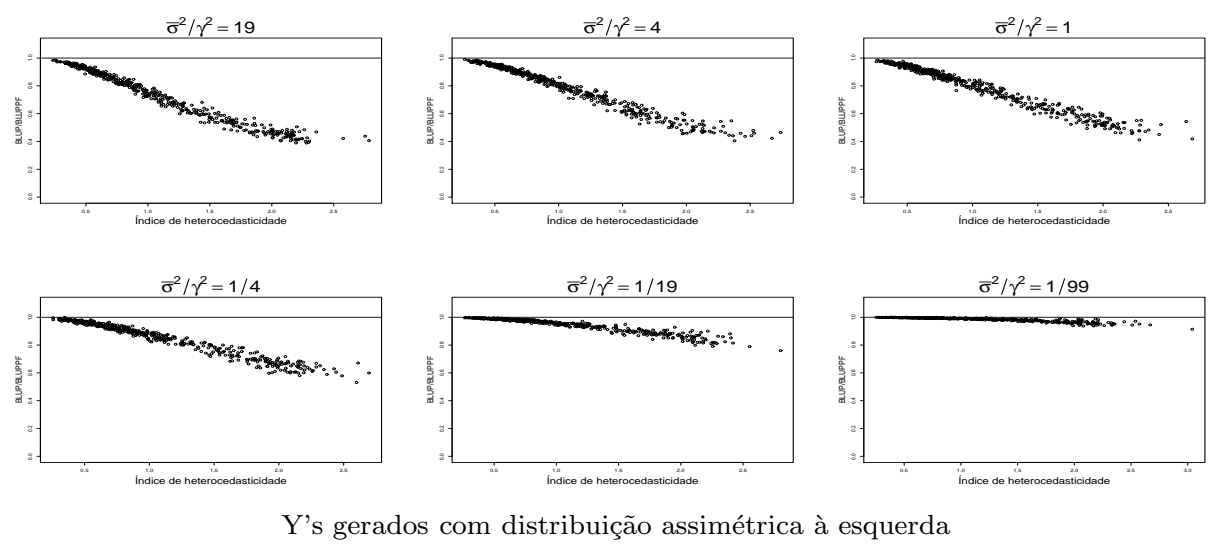

Y's gerados com distribuição assimétrica à esquerda
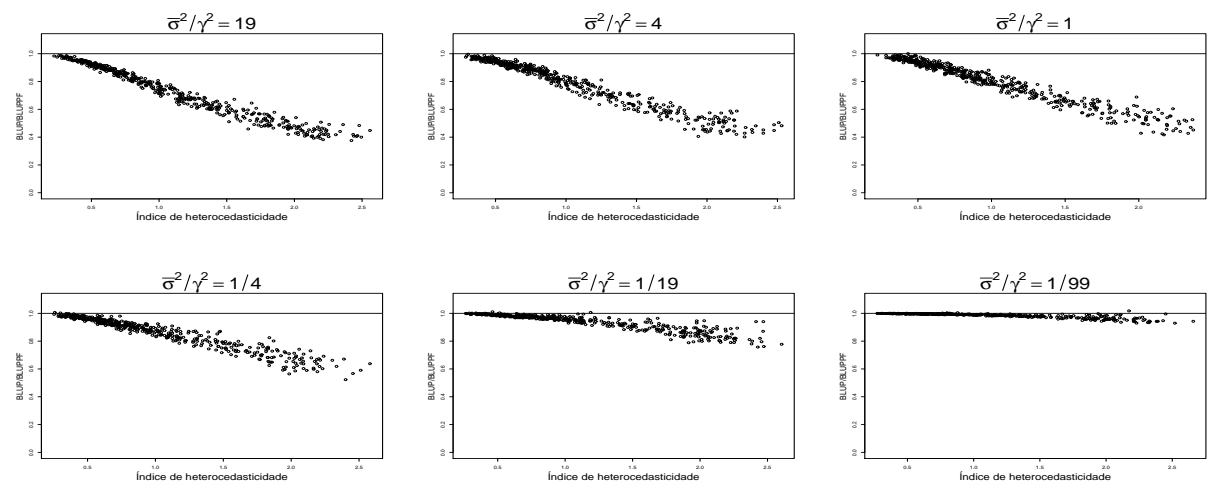

Y's gerados com distribuição assimétrica à direita
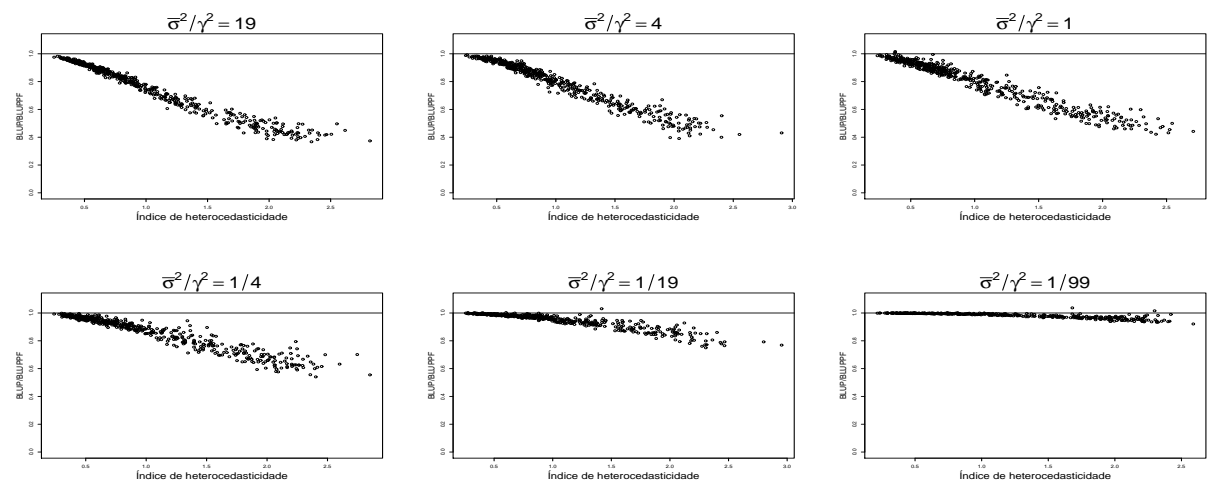
Figura F.9: Quocientes $\operatorname{EQM}\left[\hat{Q}_{i}^{(1)}\right] / \operatorname{EMQ}\left[\hat{Q}_{i}^{(2)}\right]$ sob erros de medida endógenos com distribuição uniforme, $N=300$ e $n=100$.

Y's gerados com distribuição uniforme
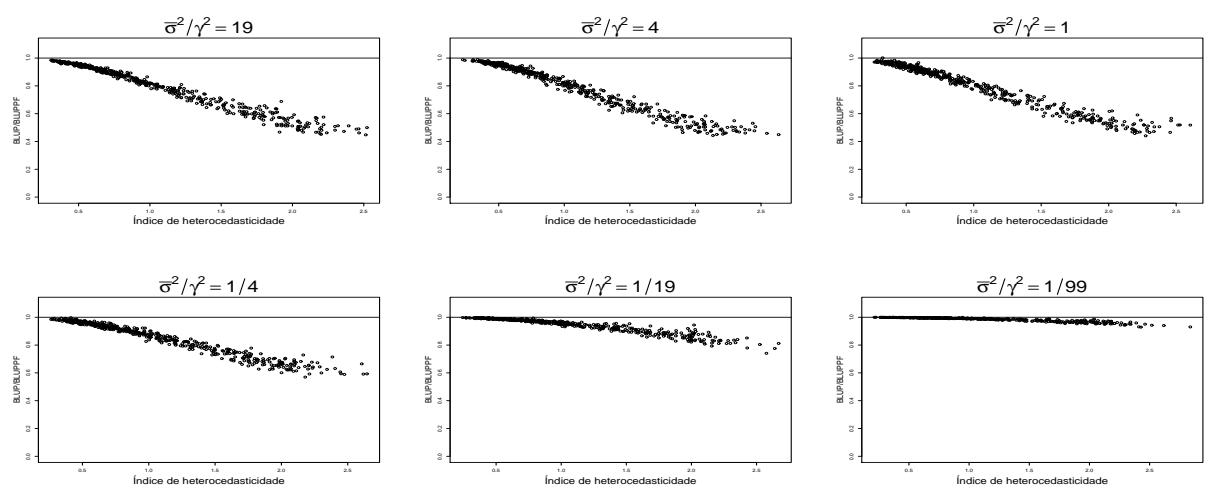

Y's gerados com distribuição assimétrica à esquerda
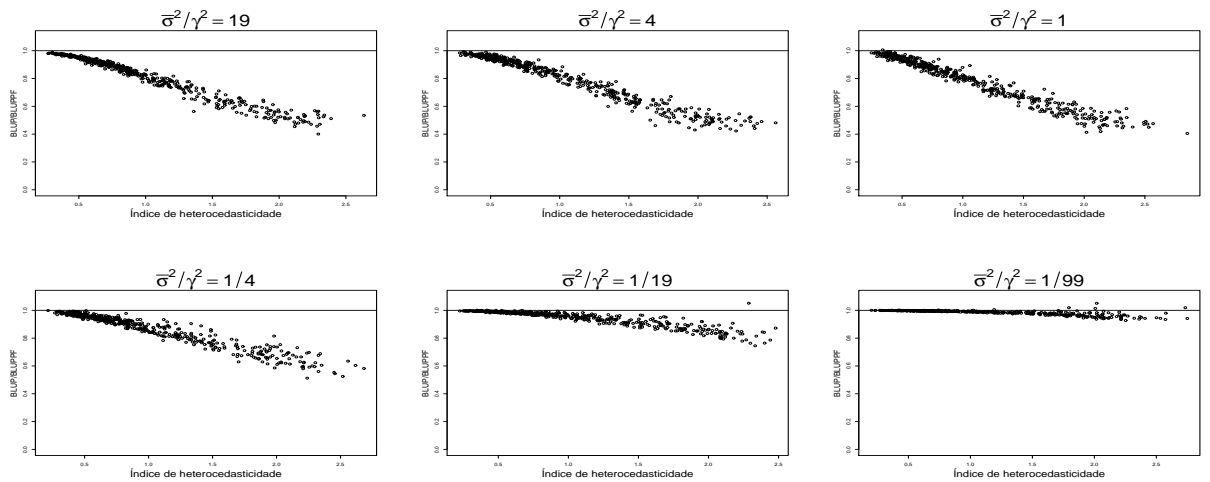

Y's gerados com distribuição assimétrica à direita
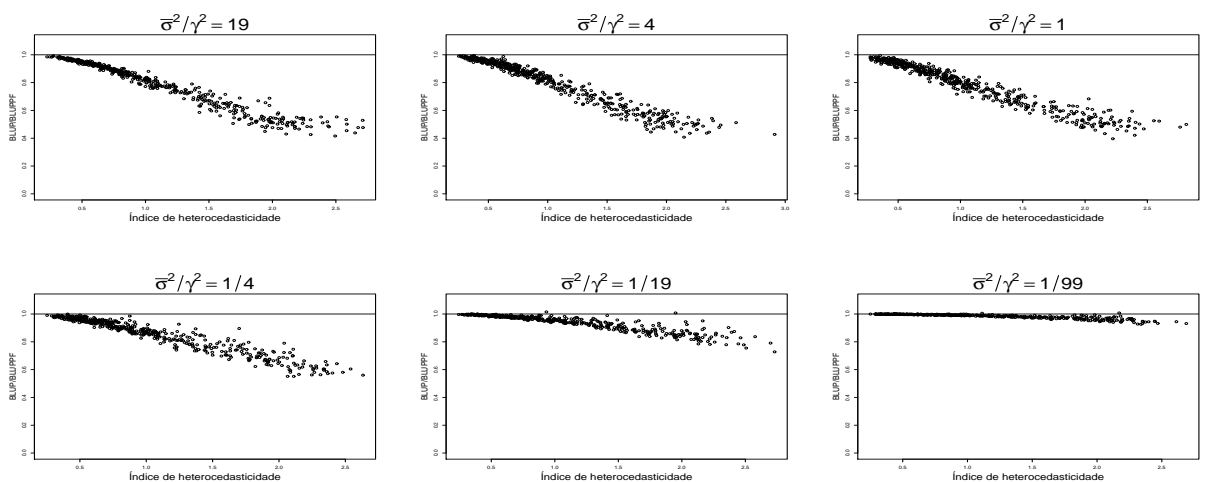
Apêndice G

Quocientes EQM $\left[\hat{Q}_{i}^{(1)}\right] / \operatorname{EMQ}\left[\hat{Q}_{i}^{(2)}\right]$ sob erros de medida endógenos com distribuição beta simétrica 
Figura G.1: Quocientes EQM $\left[\hat{Q}_{i}^{(1)}\right] / \operatorname{EMQ}\left[\hat{Q}_{i}^{(2)}\right]$ sob erros de medida endógenos com distribuição beta simétrica, $N=50$ e $n=5$.

Y's gerados com distribuição uniforme
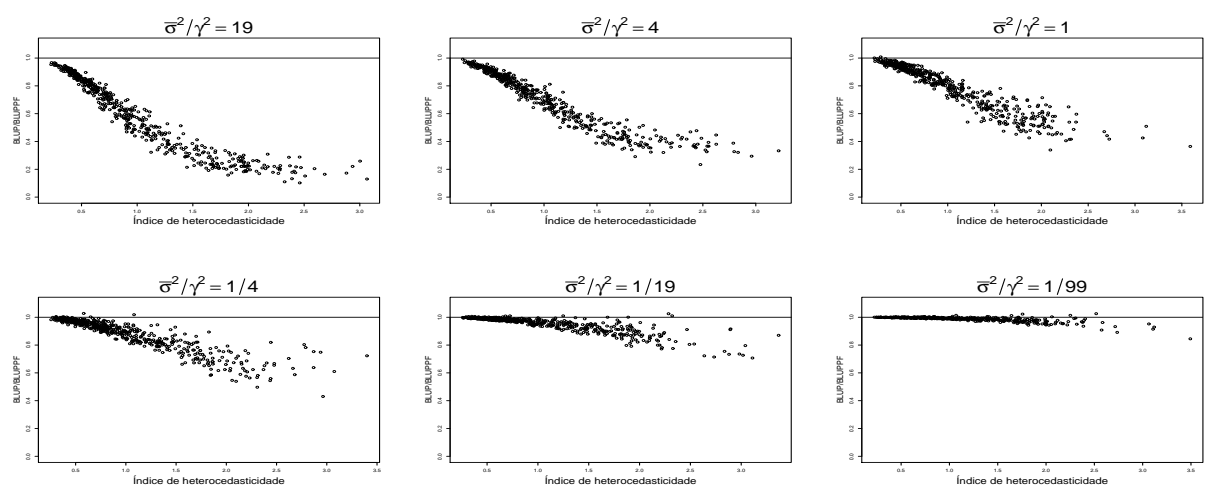

Y's gerados com distribuição assimétrica à esquerda
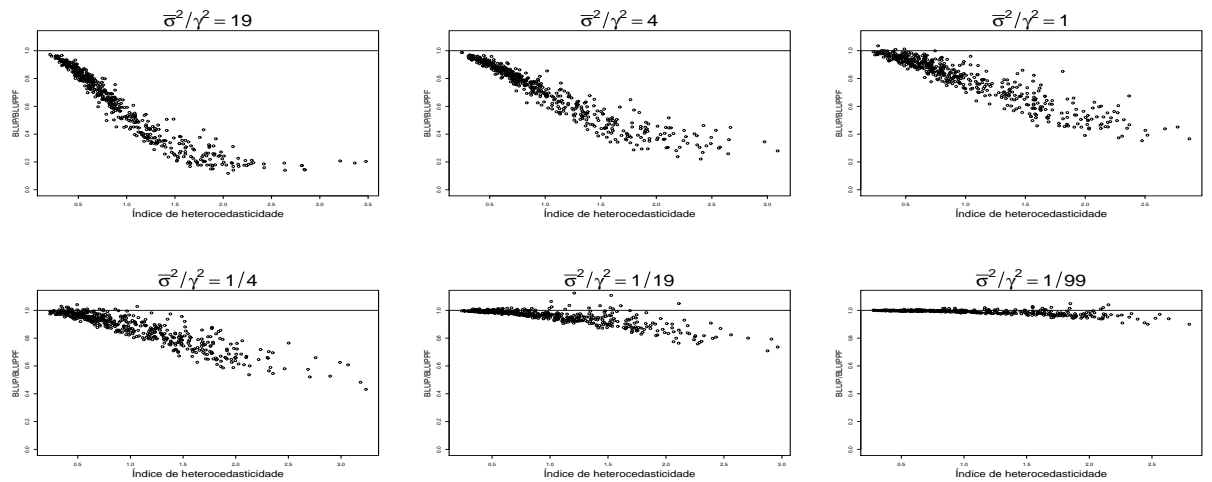

Y's gerados com distribuição assimétrica à direita
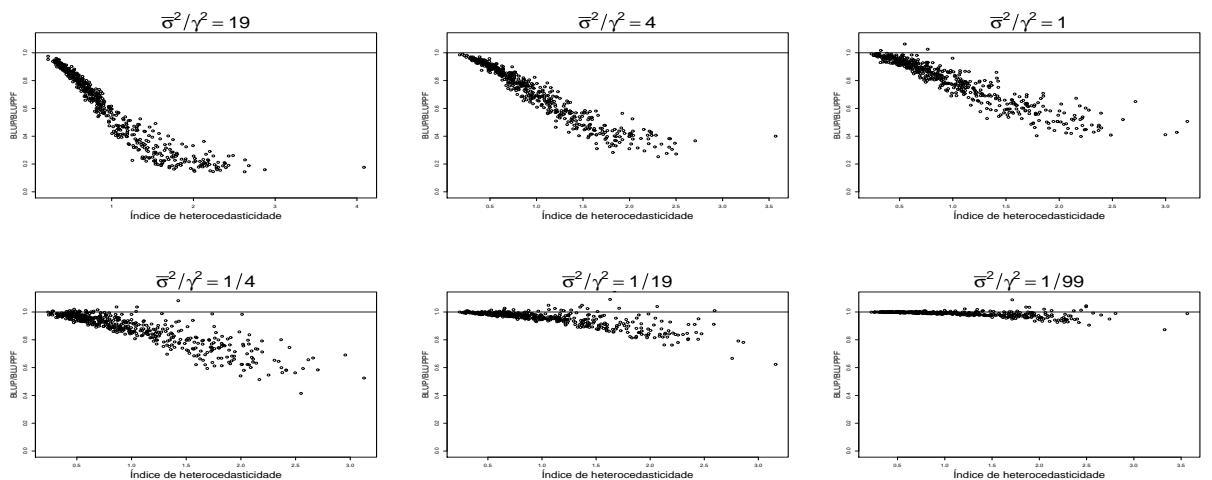
Figura G.2: Quocientes EQM $\left[\hat{Q}_{i}^{(1)}\right] / \operatorname{EMQ}\left[\hat{Q}_{i}^{(2)}\right]$ sob erros de medida endógenos com distribuição beta simétrica, $N=50$ e $n=10$.

Y's gerados com distribuição uniforme
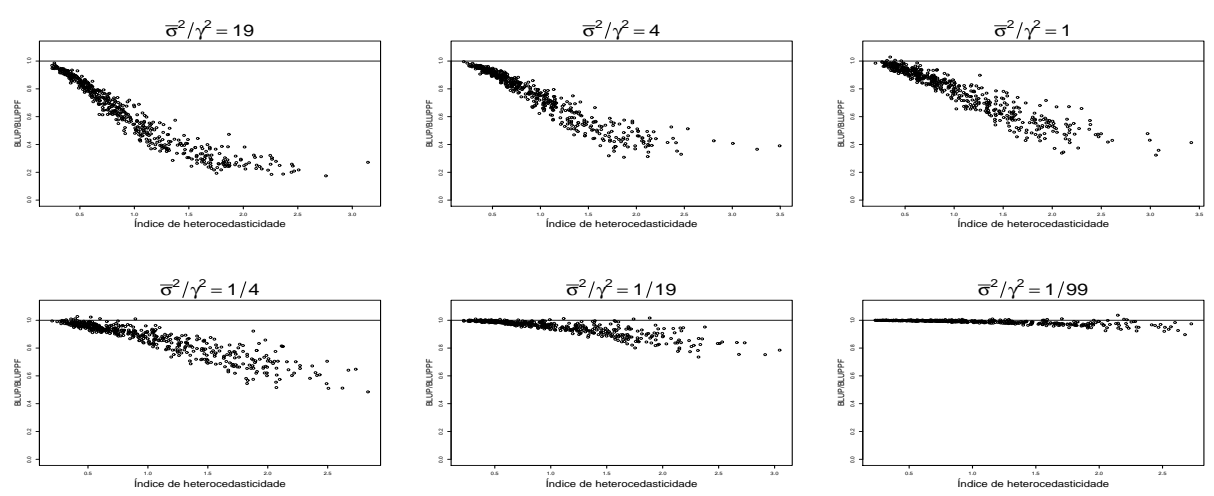

Y's gerados com distribuição assimétrica à esquerda
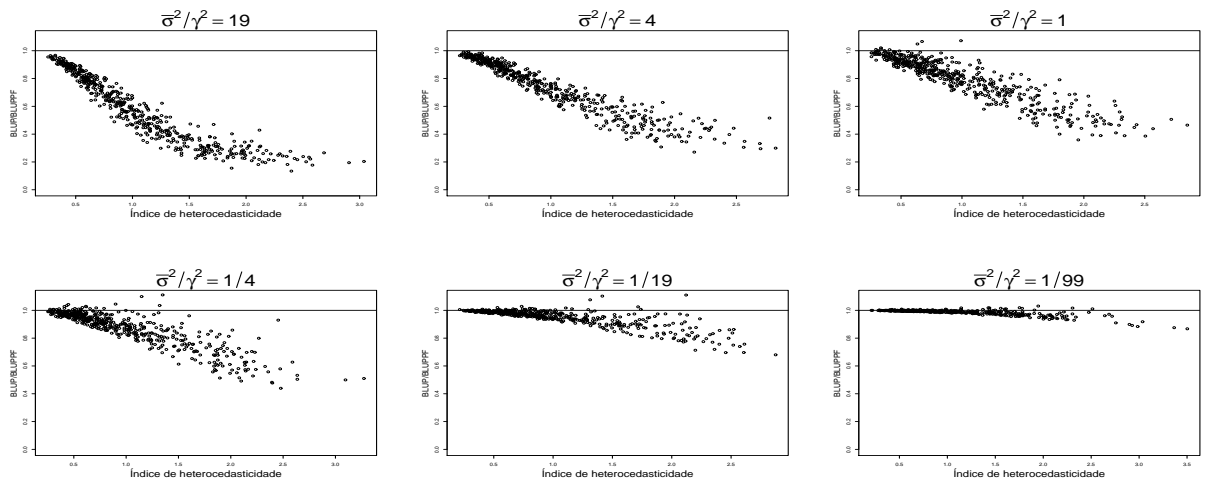

Y's gerados com distribuição assimétrica à direita
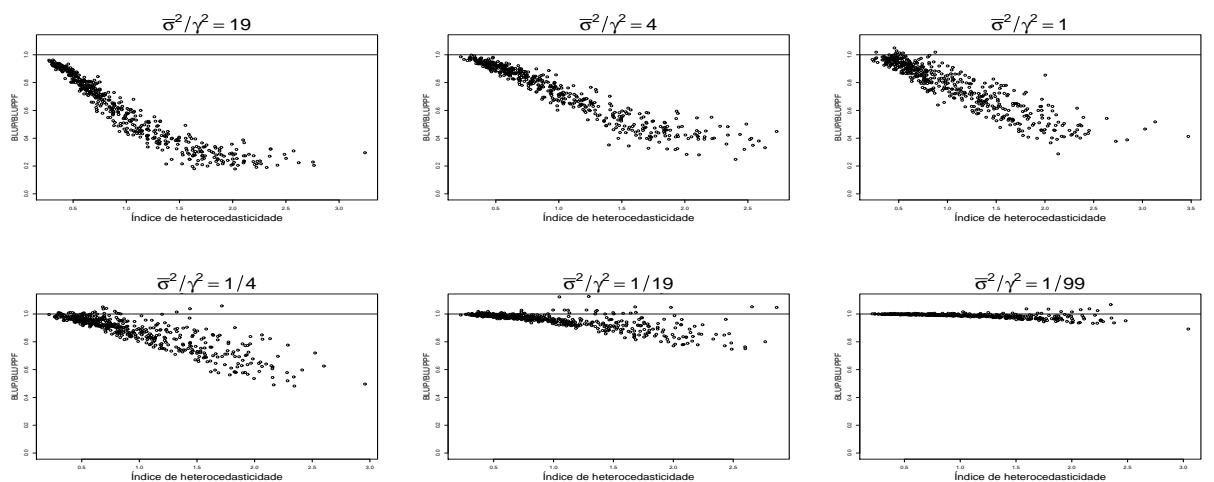
Figura G.3: Quocientes EQM $\left[\hat{Q}_{i}^{(1)}\right] / \operatorname{EMQ}\left[\hat{Q}_{i}^{(2)}\right]$ sob erros de medida endógenos com distribuição beta simétrica, $N=50$ e $n=25$.

Y's gerados com distribuição uniforme
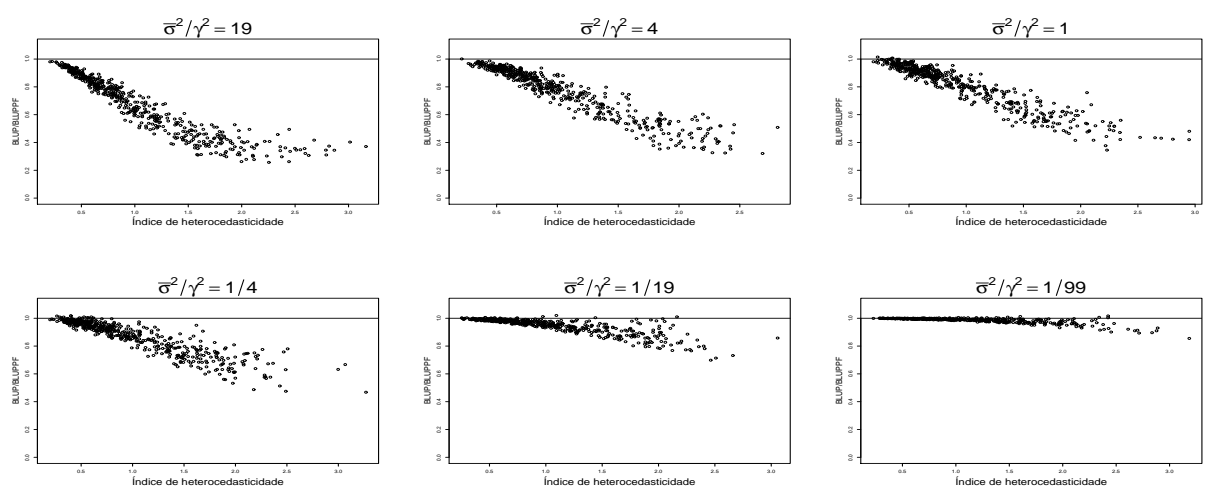

Y's gerados com distribuição assimétrica à esquerda
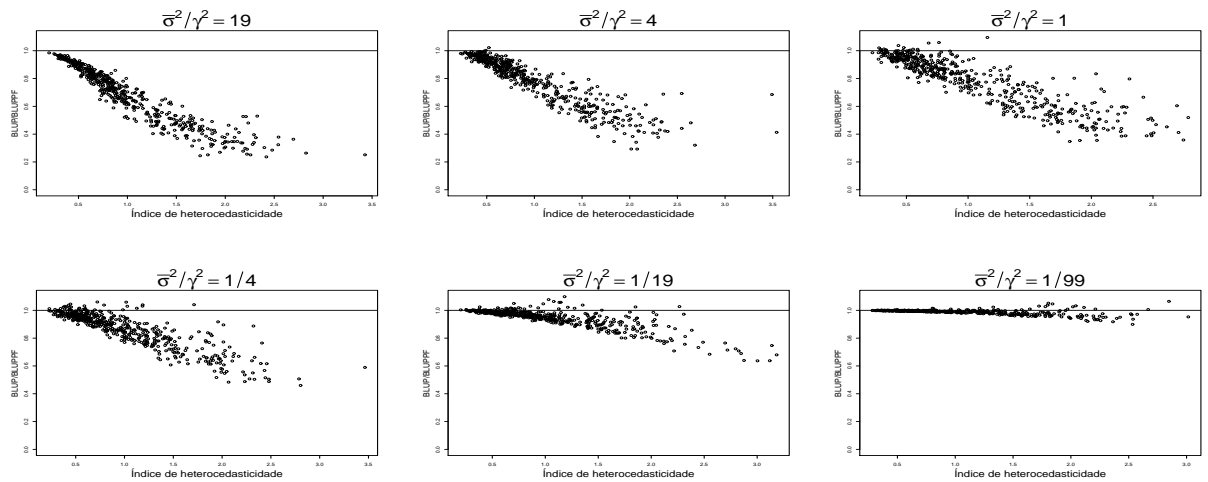

Y's gerados com distribuição assimétrica à direita
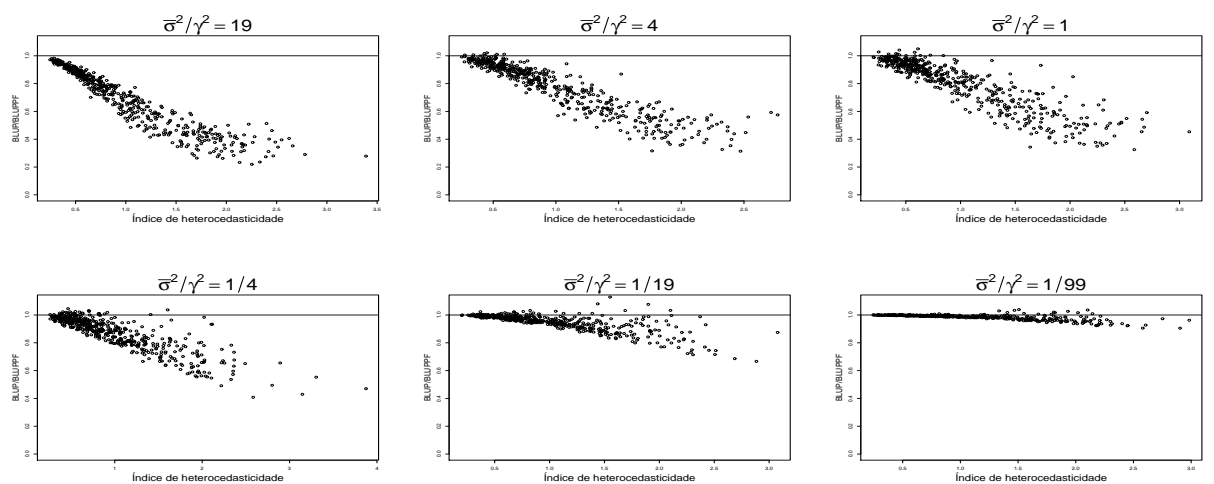
Figura G.4: Quocientes EQM $\left[\hat{Q}_{i}^{(1)}\right] / \operatorname{EMQ}\left[\hat{Q}_{i}^{(2)}\right]$ sob erros de medida endógenos com distribuição beta simétrica, $N=100$ e $n=10$.

Y's gerados com distribuição uniforme
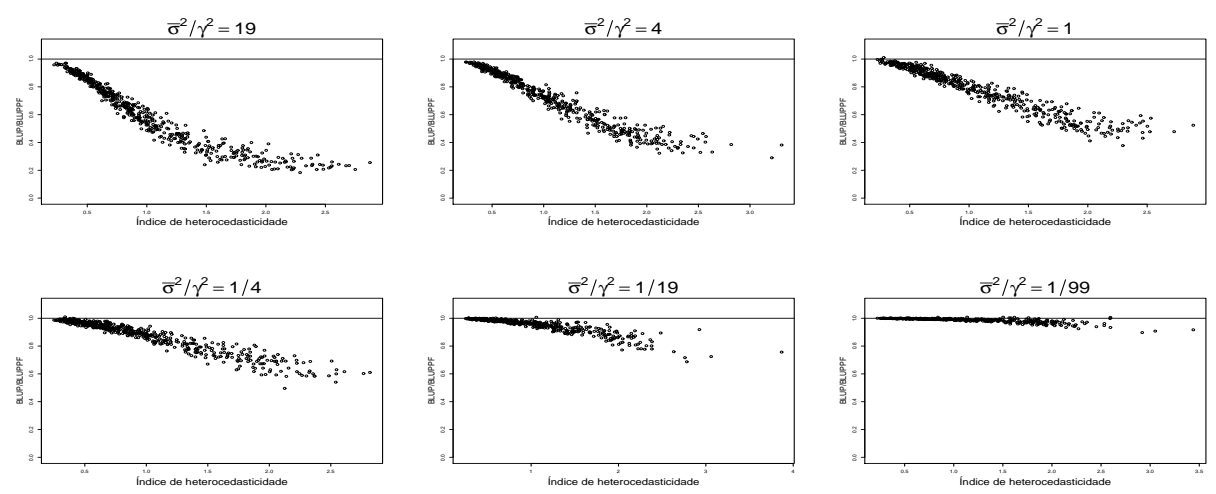

Y's gerados com distribuição assimétrica à esquerda
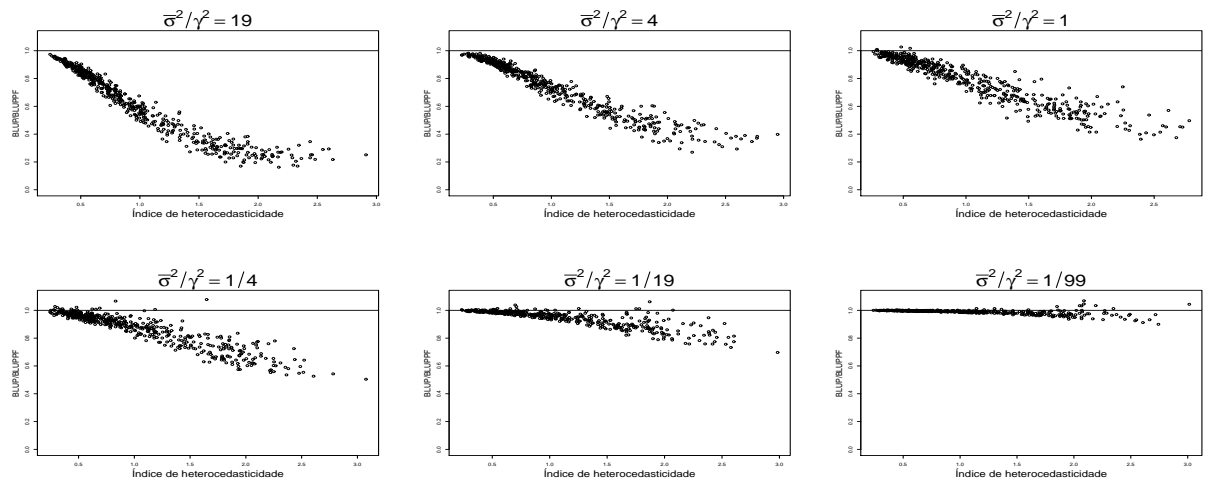

Y's gerados com distribuição assimétrica à direita
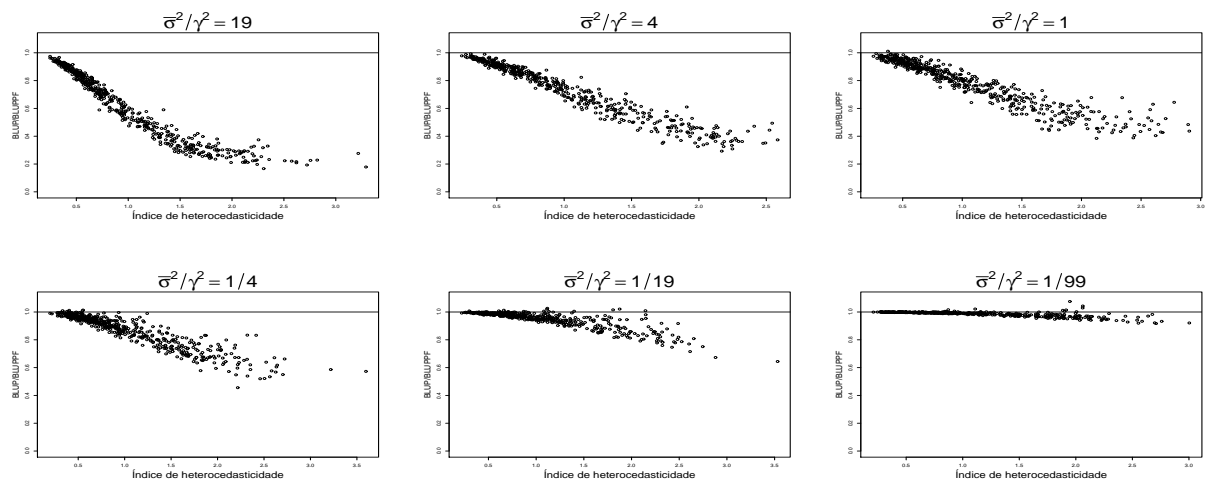
Figura G.5: Quocientes EQM $\left[\hat{Q}_{i}^{(1)}\right] / \operatorname{EMQ}\left[\hat{Q}_{i}^{(2)}\right]$ sob erros de medida endógenos com distribuição beta simétrica, $N=100$ e $n=30$.

Y's gerados com distribuição uniforme
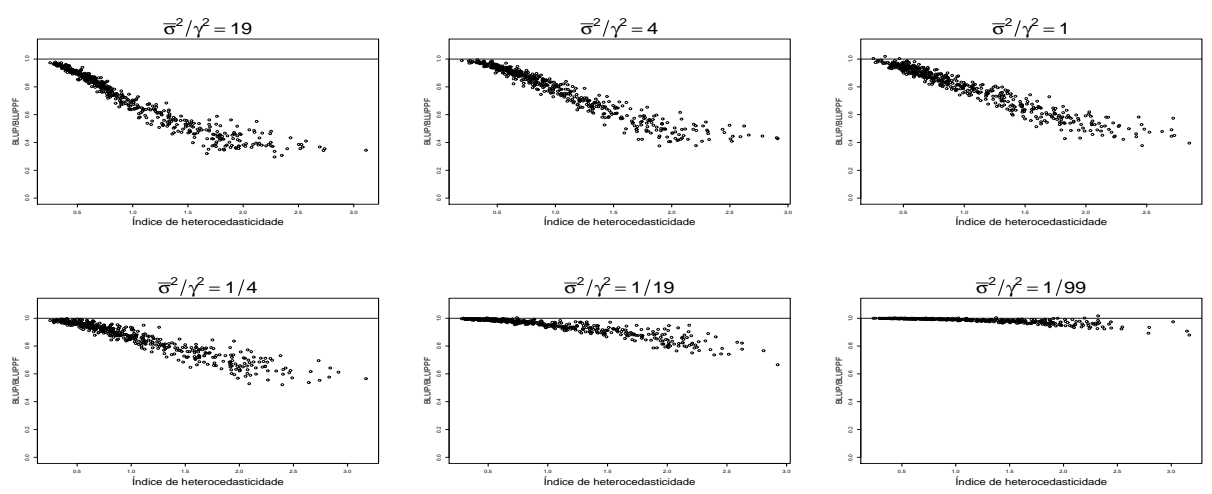

Y's gerados com distribuição assimétrica à esquerda
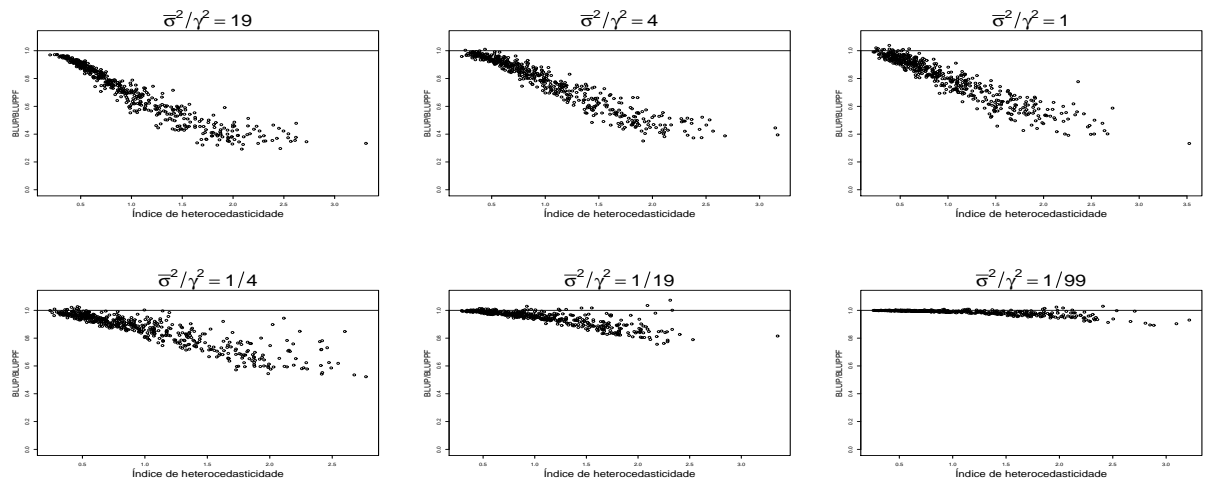

Y's gerados com distribuição assimétrica à direita
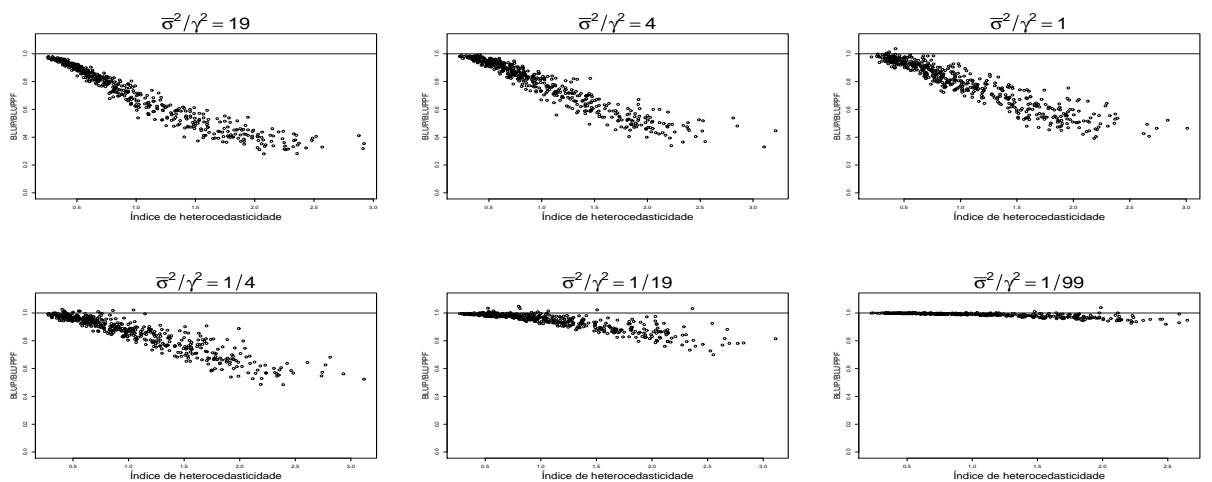
Figura G.6: Quocientes EQM $\left[\hat{Q}_{i}^{(1)}\right] / \operatorname{EMQ}\left[\hat{Q}_{i}^{(2)}\right]$ sob erros de medida endógenos com distribuição beta simétrica, $N=100$ e $n=50$.

Y's gerados com distribuição uniforme
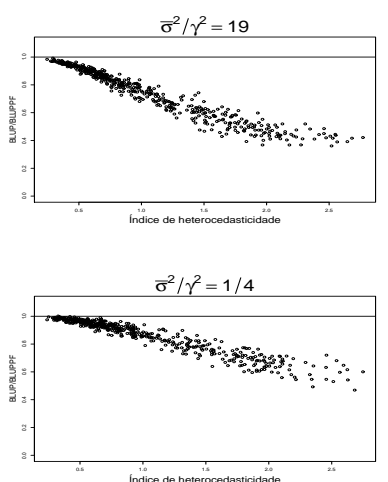

Y's gerados com distribuição assimétrica à esquerda
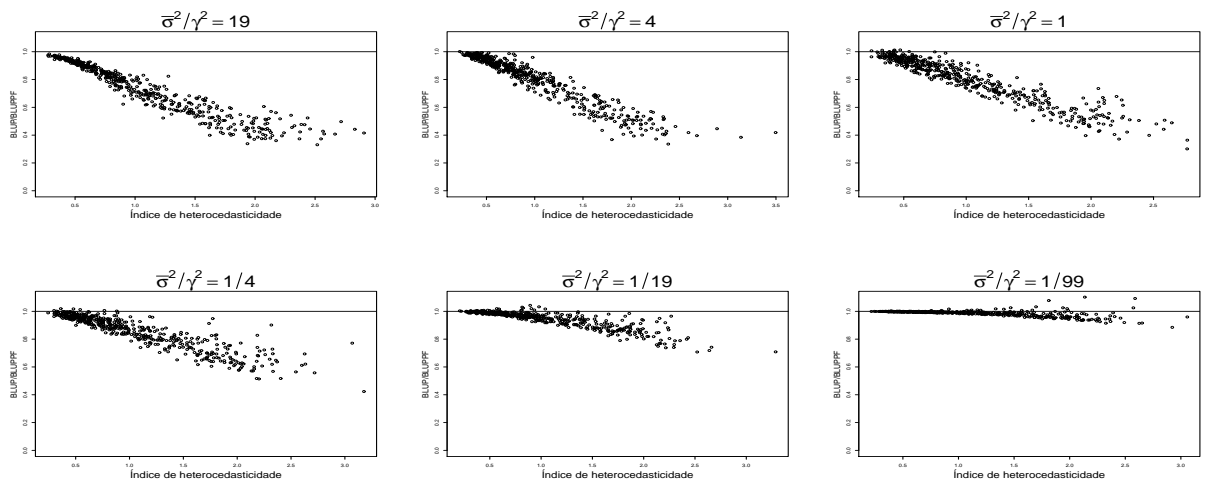

Y's gerados com distribuição assimétrica à direita
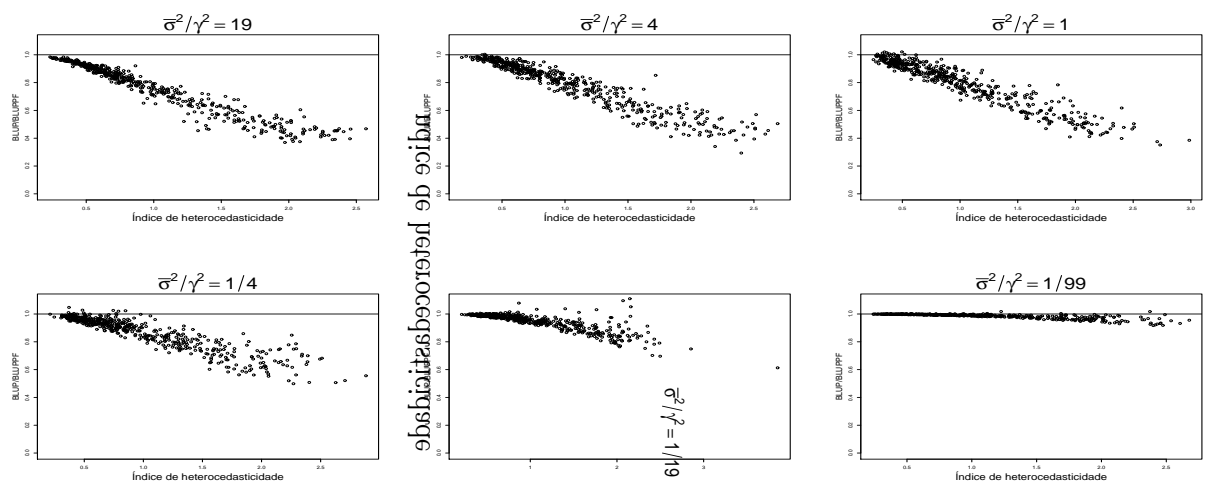
Figura G.7: Quocientes EQM $\left[\hat{Q}_{i}^{(1)}\right] / \operatorname{EMQ}\left[\hat{Q}_{i}^{(2)}\right]$ sob erros de medida endógenos com distribuição beta simétrica, $N=300$ e $n=30$.

Y's gerados com distribuição uniforme
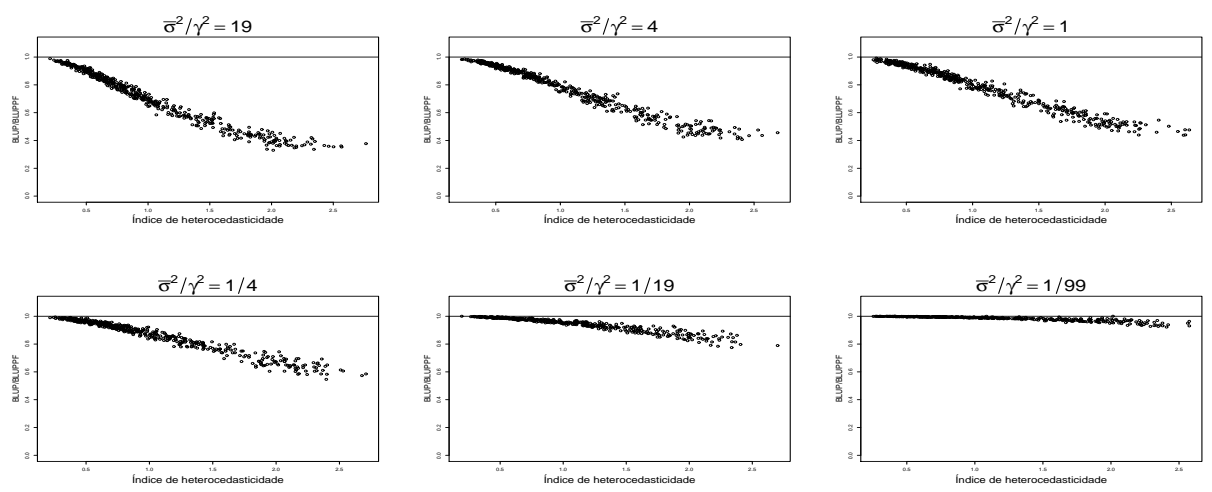

Y's gerados com distribuição assimétrica à esquerda
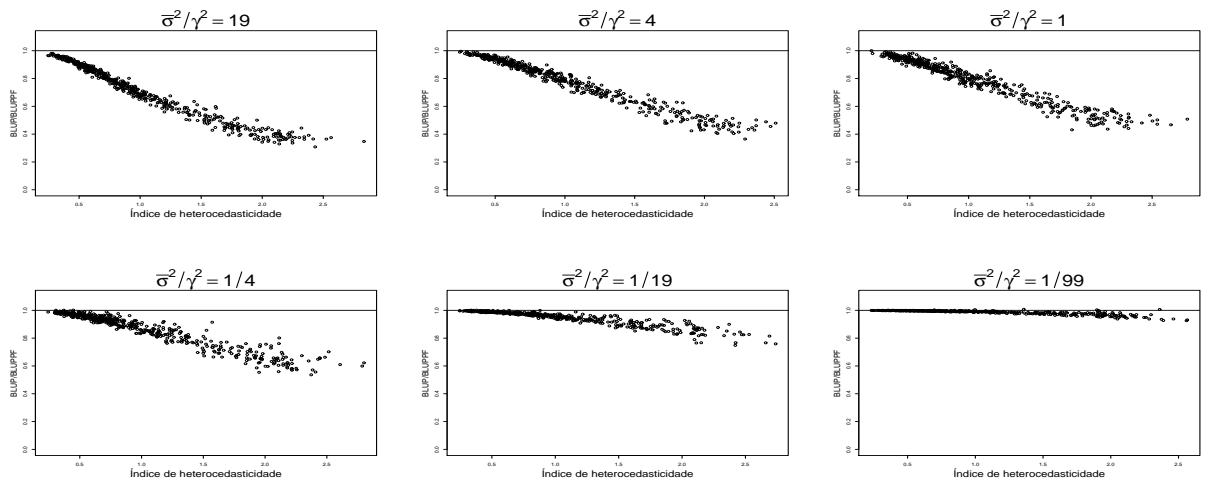

Y's gerados com distribuição assimétrica à direita
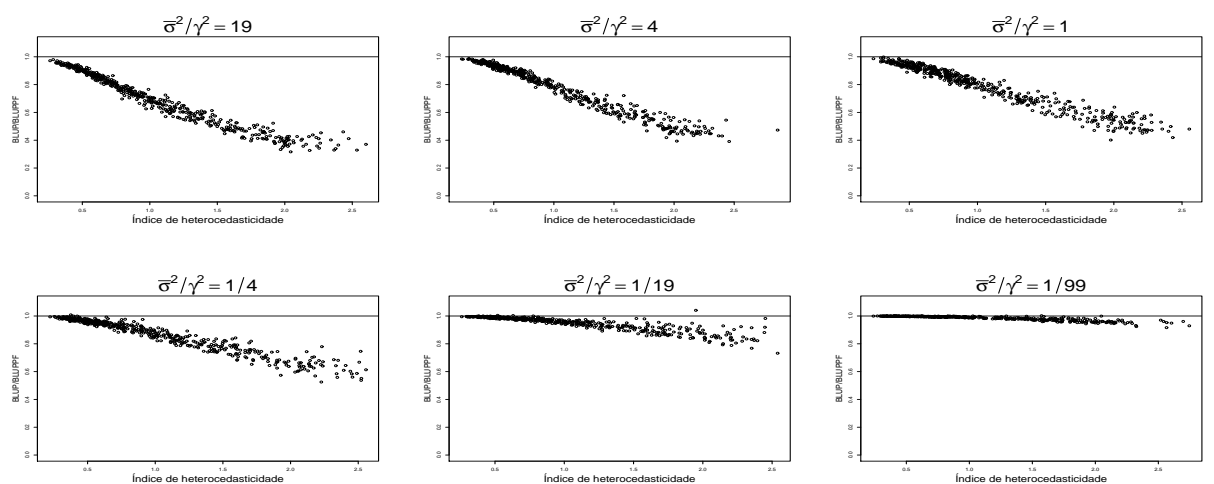
Figura G.8: Quocientes EQM $\left[\hat{Q}_{i}^{(1)}\right] / \operatorname{EMQ}\left[\hat{Q}_{i}^{(2)}\right]$ sob erros de medida endógenos com distribuição beta simétrica, $N=300$ e $n=50$.

Y's gerados com distribuição uniforme
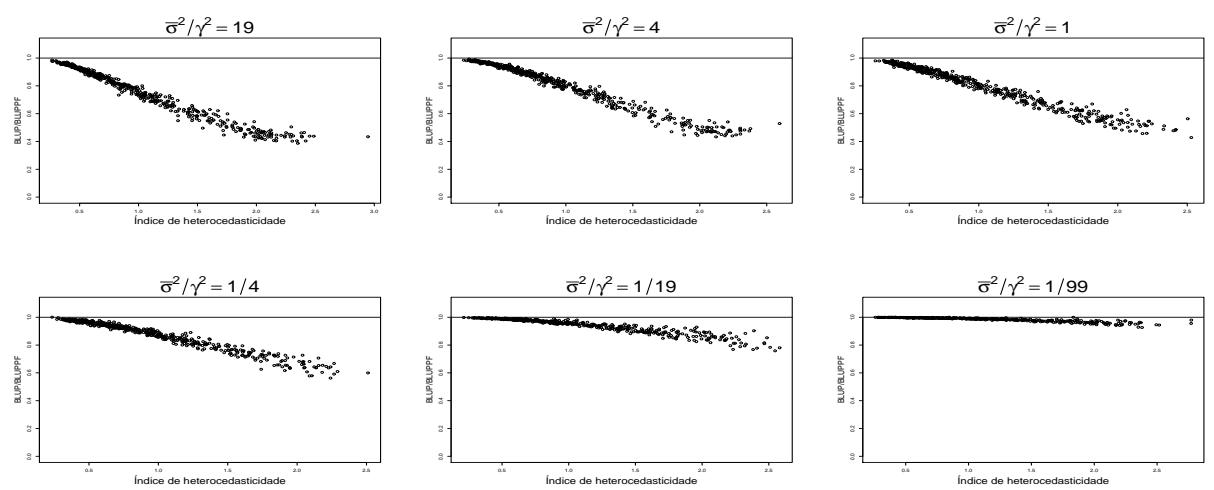

Y's gerados com distribuição assimétrica à esqBSUErda
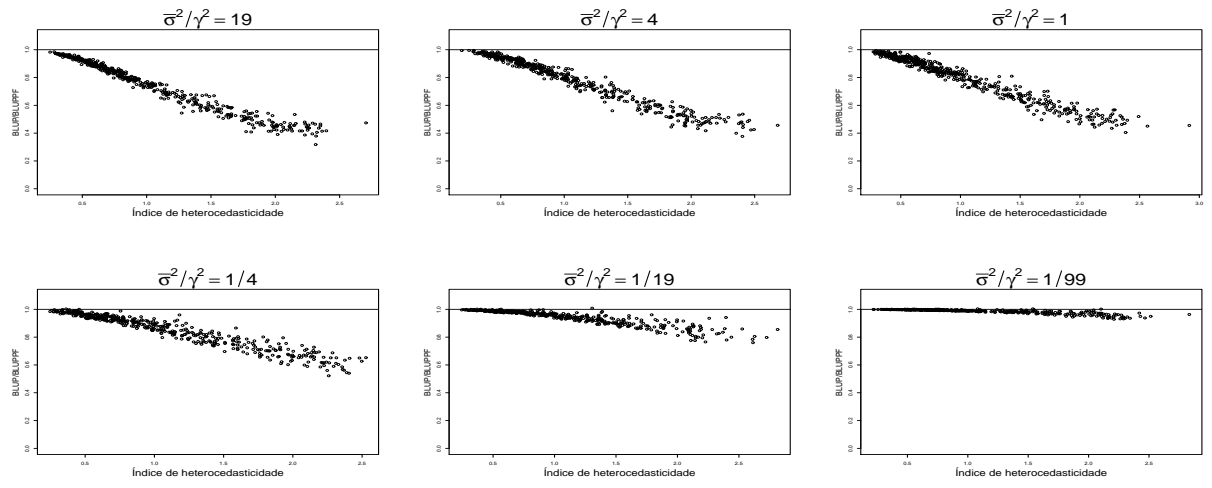

Y's gerados com distribuição assimétrica à direita
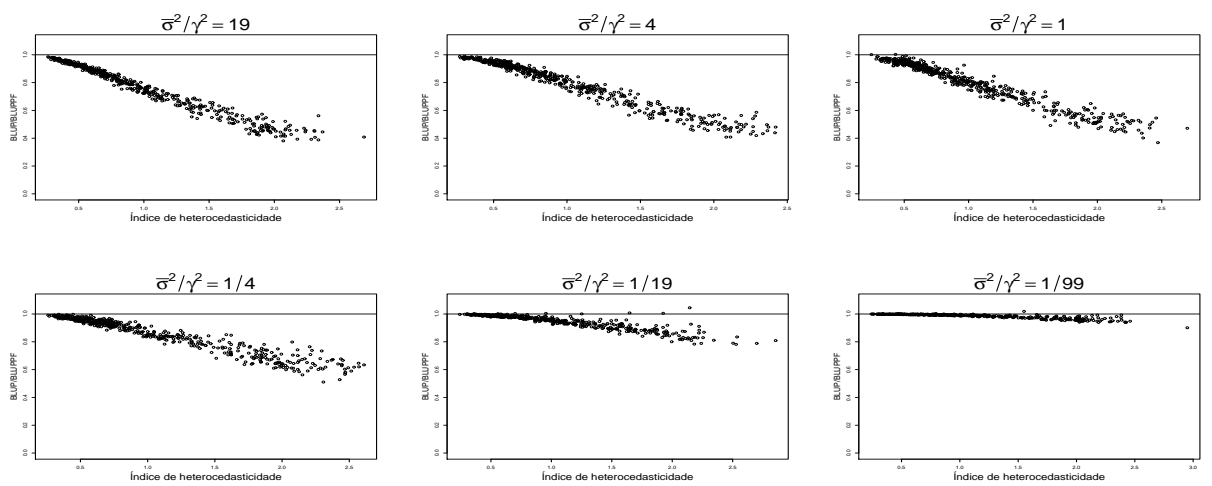
Figura G.9: Quocientes EQM $\left[\hat{Q}_{i}^{(1)}\right] / \operatorname{EMQ}\left[\hat{Q}_{i}^{(2)}\right]$ sob erros de medida endógenos com distribuição beta simétrica, $N=300$ e $n=100$.

Y's gerados com distribuição uniforme
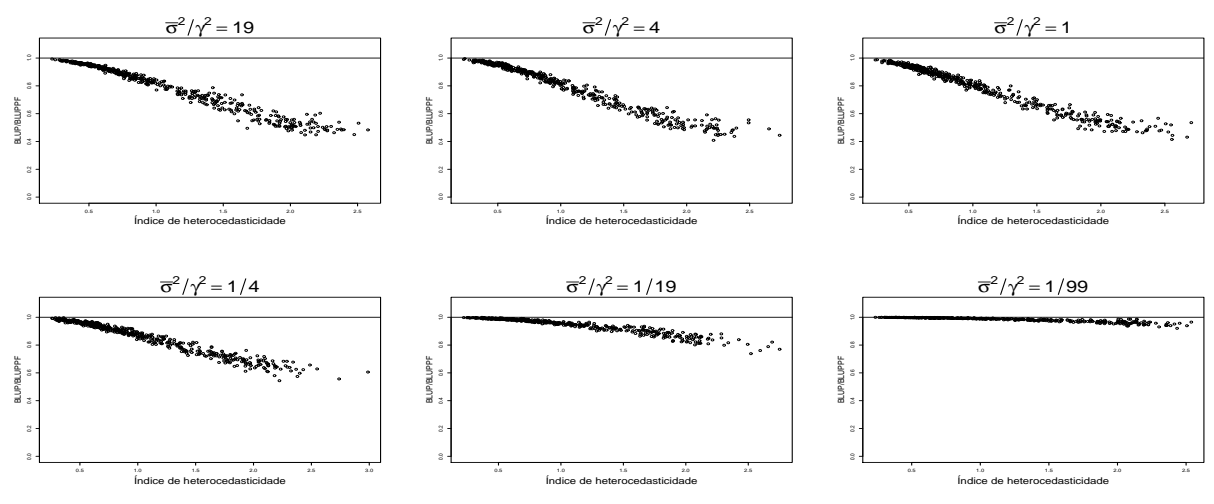

Y's gerados com distribuição assimétrica à esquerda
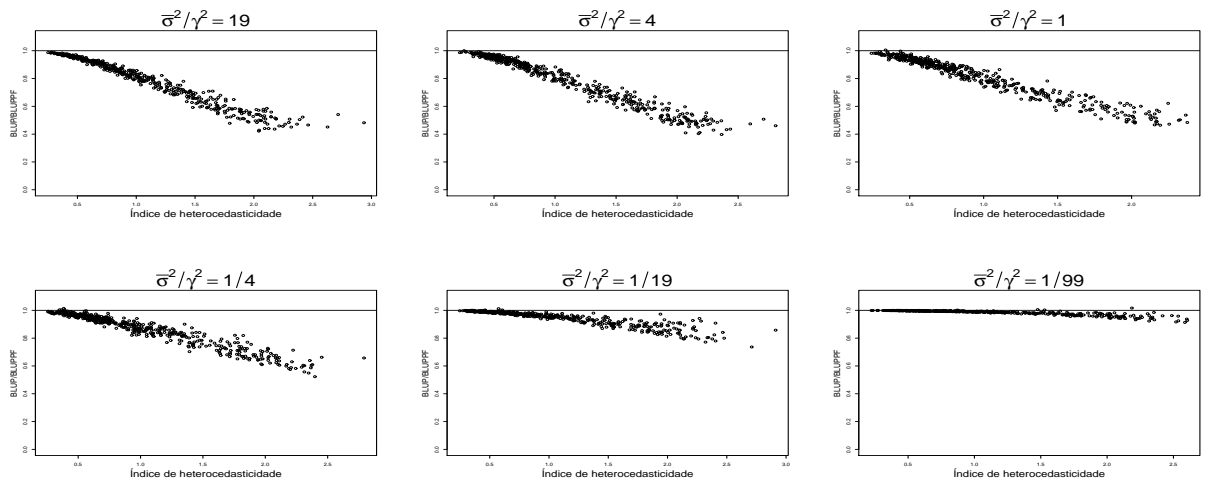

Y's gerados com distribuição assimétrica à direita
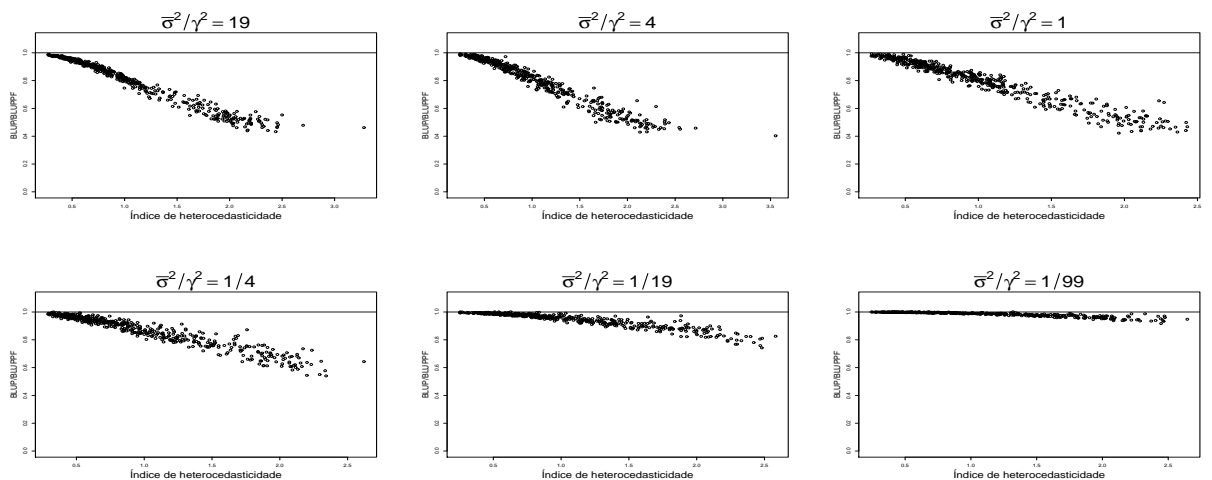
Apêndice $\mathbf{H}$

Quocientes EQM $\left[\hat{Q}_{i}^{(1)}\right] / \mathbf{E M Q}\left[\hat{Q}_{i}^{(2)}\right]$ sob erros de medida endógenos com distribuição beta assimétrica 
Figura H.1: Quocientes EQM $\left[\hat{Q}_{i}^{(1)}\right] / \operatorname{EMQ}\left[\hat{Q}_{i}^{(2)}\right]$ sob erros de medida endógenos com distribuição beta assimétrica, $N=50$ e $n=5$.

Y's gerados com distribuição uniforme
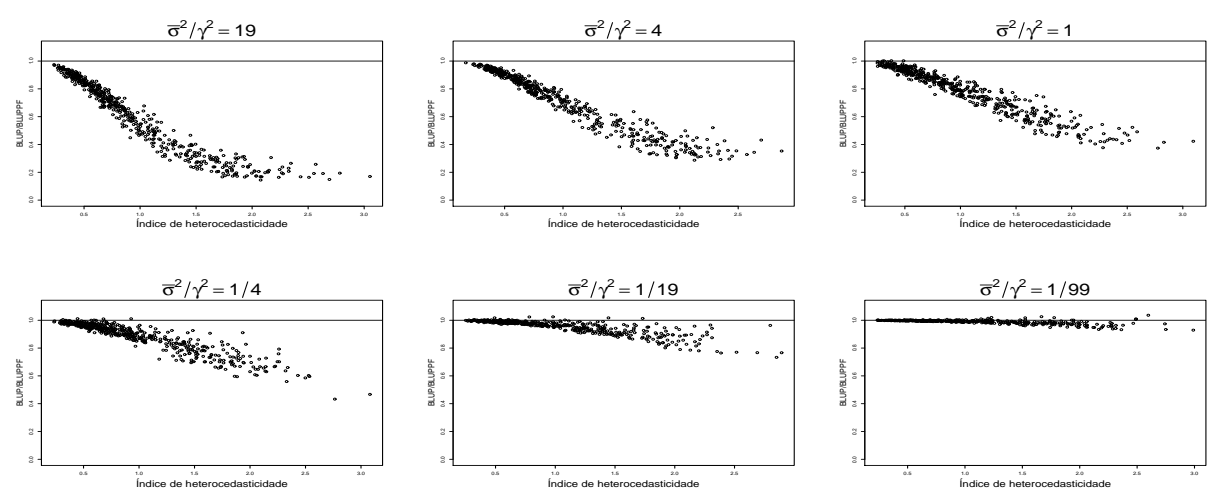

Y's gerados com distribuição assimétrica à esquerda
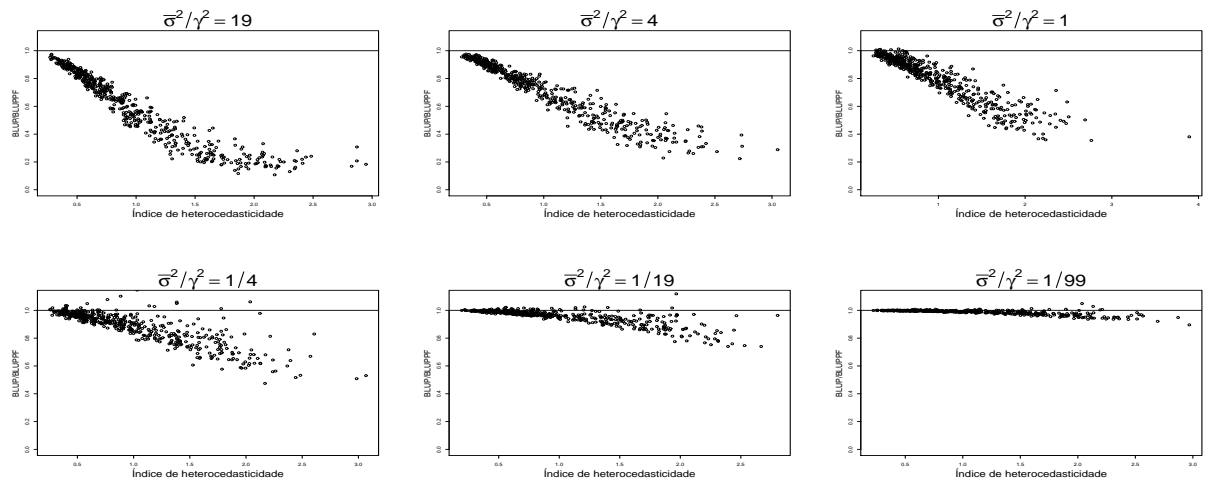

Y's gerados com distribuição assimétrica à direita
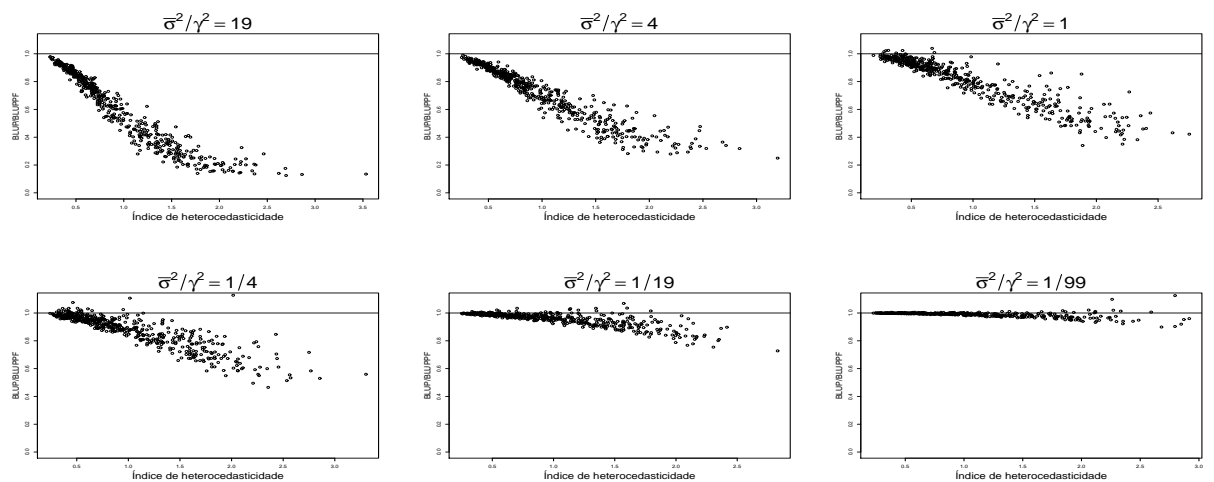
Figura H.2: Quocientes $\operatorname{EQM}\left[\hat{Q}_{i}^{(1)}\right] / \operatorname{EMQ}\left[\hat{Q}_{i}^{(2)}\right]$ sob erros de medida endógenos com distribuição beta assimétrica, $N=50$ e $n=10$.

Y's gerados com distribuição uniforme
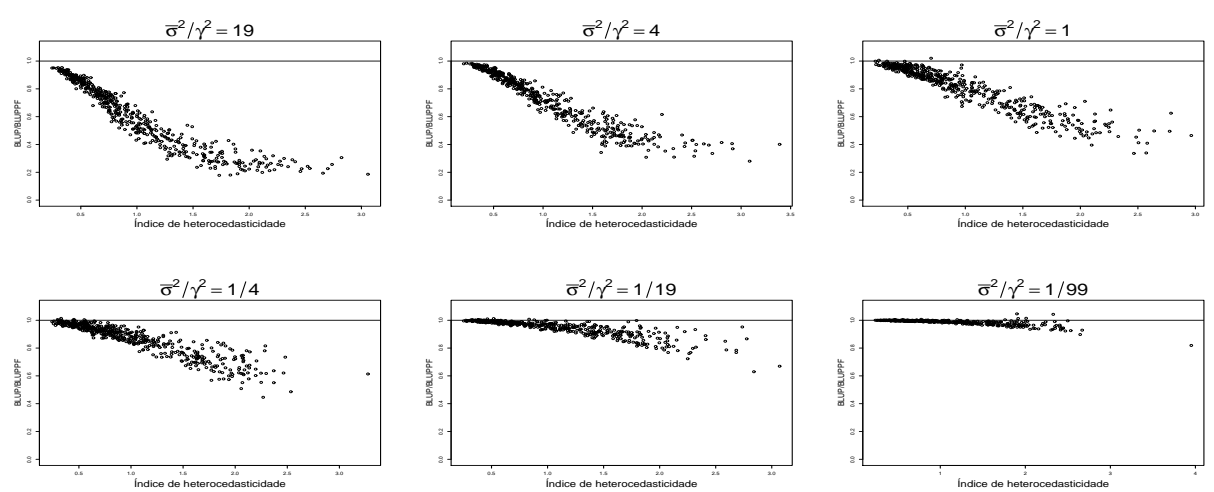

Y's gerados com distribuição assimétrica à esquerda
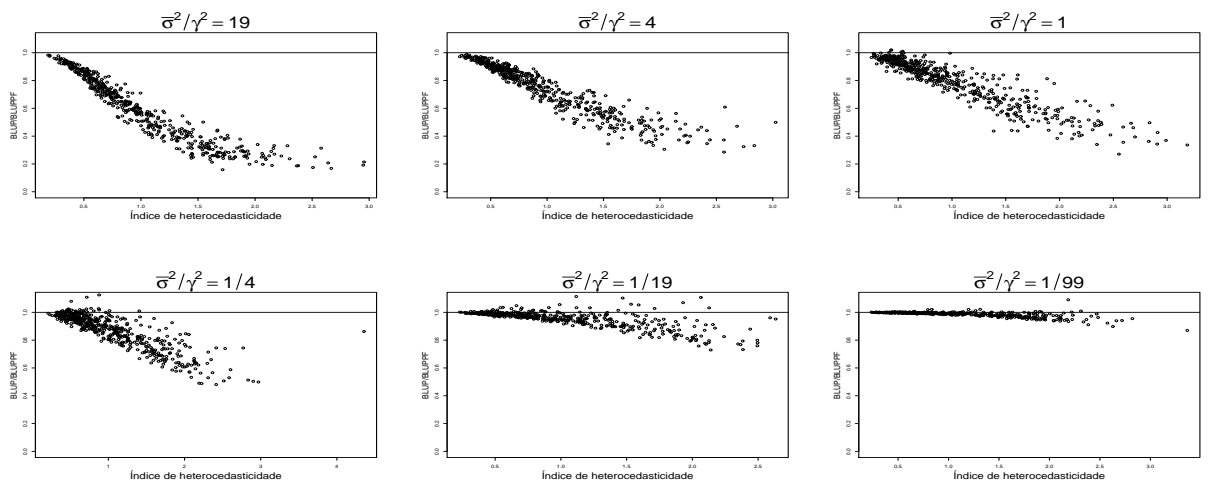

Y's gerados com distribuição assimétrica à direita
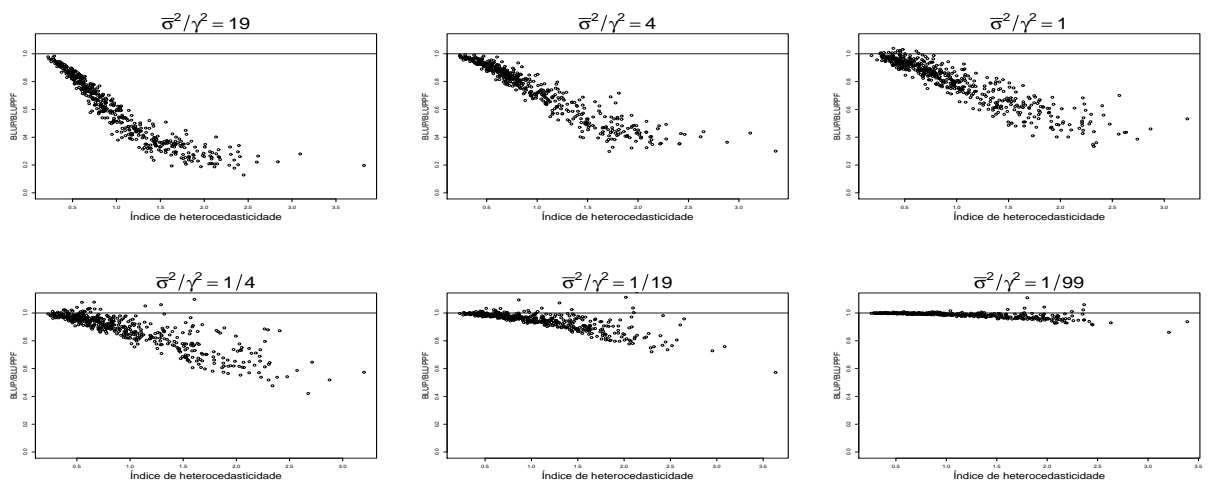
Figura H.3: Quocientes EQM $\left[\hat{Q}_{i}^{(1)}\right] / \operatorname{EMQ}\left[\hat{Q}_{i}^{(2)}\right]$ sob erros de medida endógenos com distribuição beta assimétrica, $N=50$ e $n=25$.

Y's gerados com distribuição uniforme
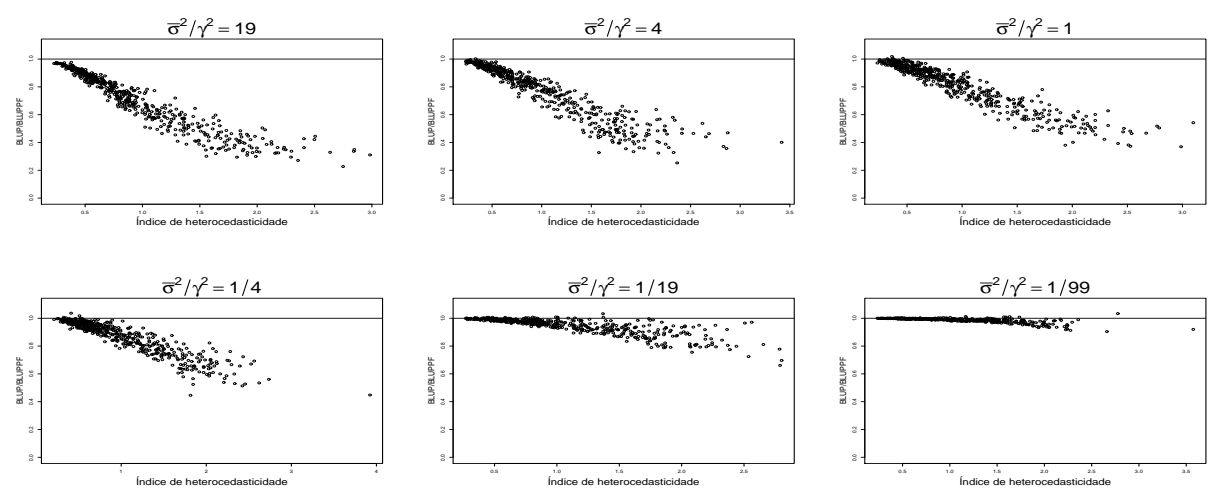

Y's gerados com distribuição assimétrica à esquerda
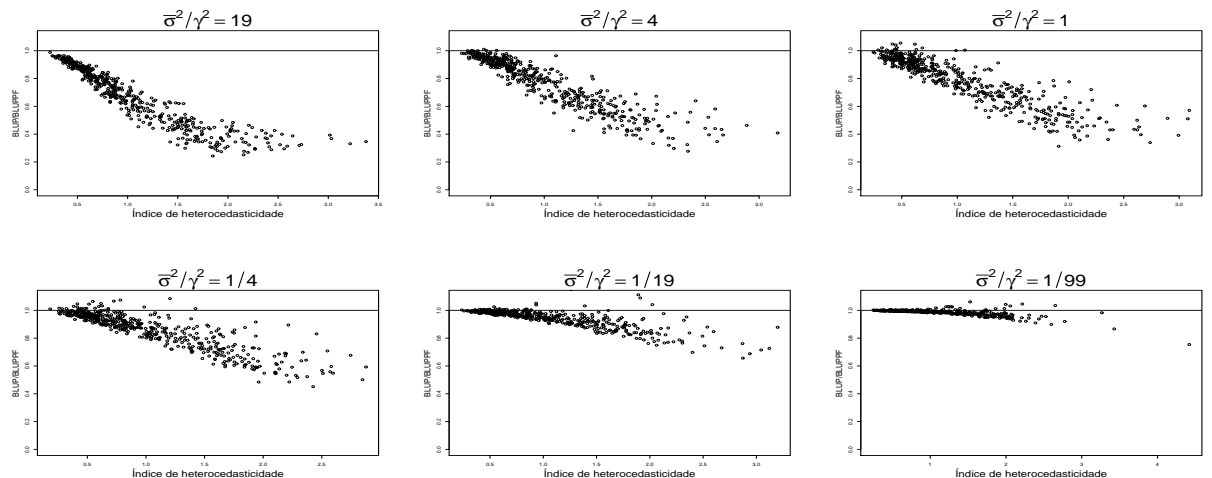

Y's gerados com distribuição assimétrica à direita
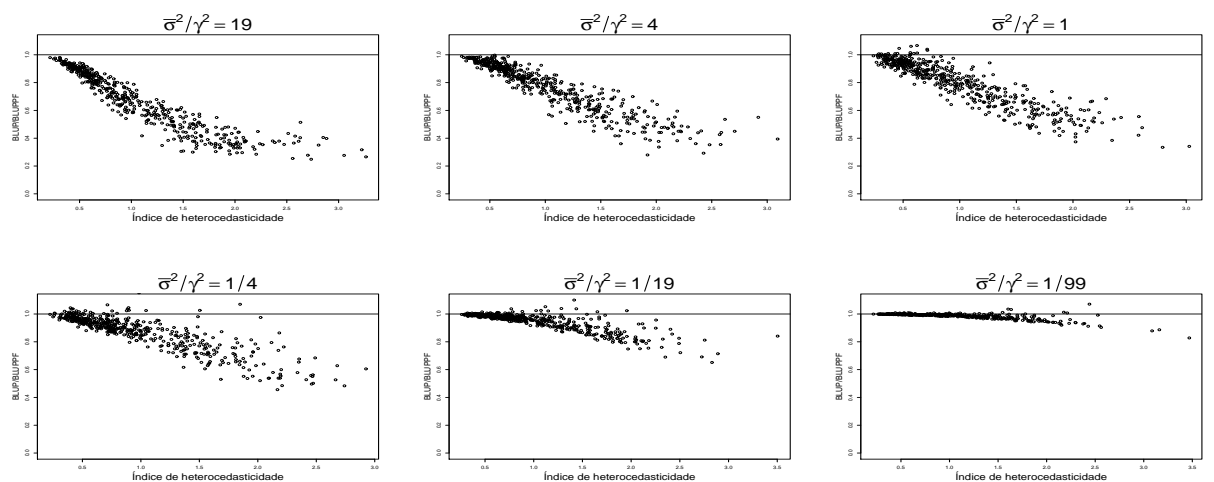
Figura H.4: Quocientes EQM $\left[\hat{Q}_{i}^{(1)}\right] / \operatorname{EMQ}\left[\hat{Q}_{i}^{(2)}\right]$ sob erros de medida endógenos com distribuição beta assimétrica, $N=100$ e $n=10$.

Y's gerados com distribuição uniforme
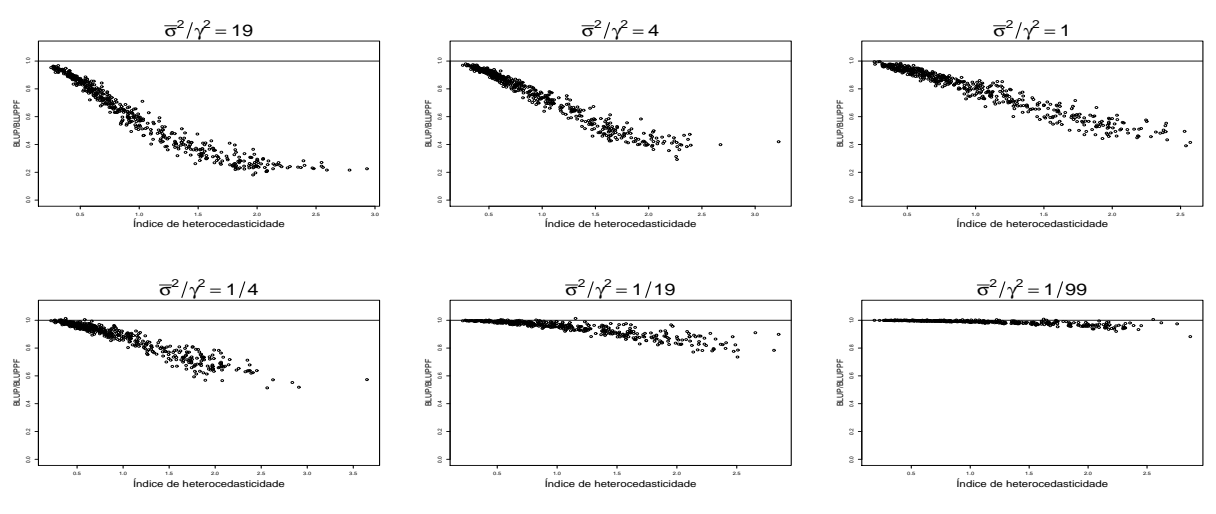

Y's gerados com distribuição assimétrica à esquerda
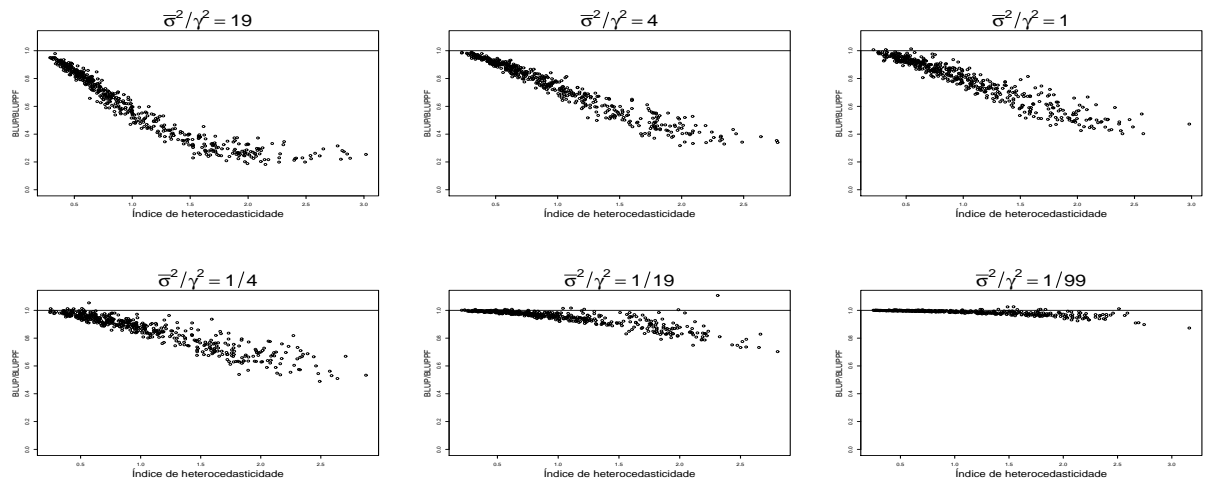

Y's gerados com distribuição assimétrica à direita
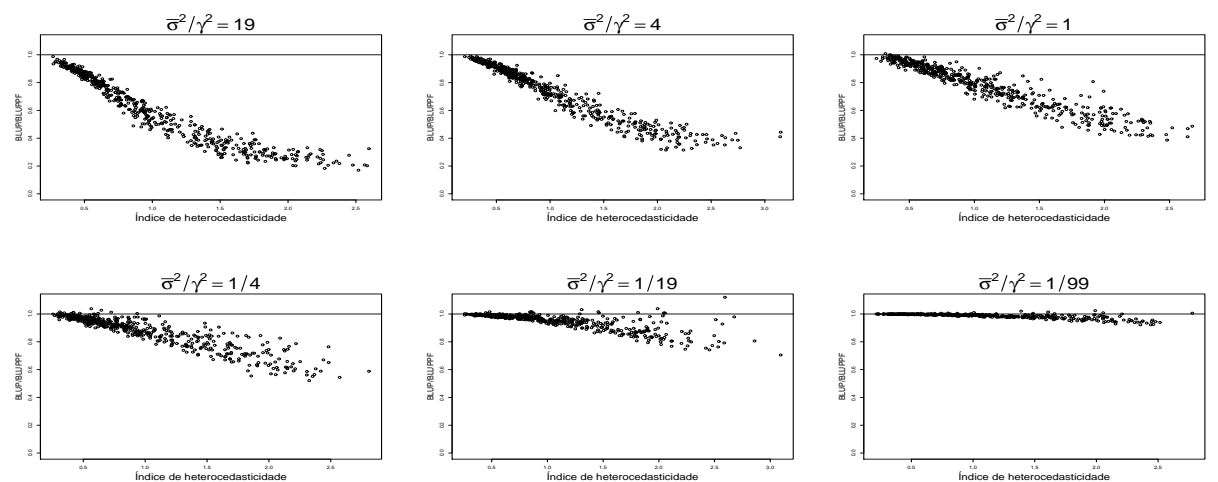
Figura H.5: Quocientes EQM $\left[\hat{Q}_{i}^{(1)}\right] / \operatorname{EMQ}\left[\hat{Q}_{i}^{(2)}\right]$ sob erros de medida endógenos com distribuição beta assimétrica, $N=100$ e $n=30$.

Y's gerados com distribuição uniforme
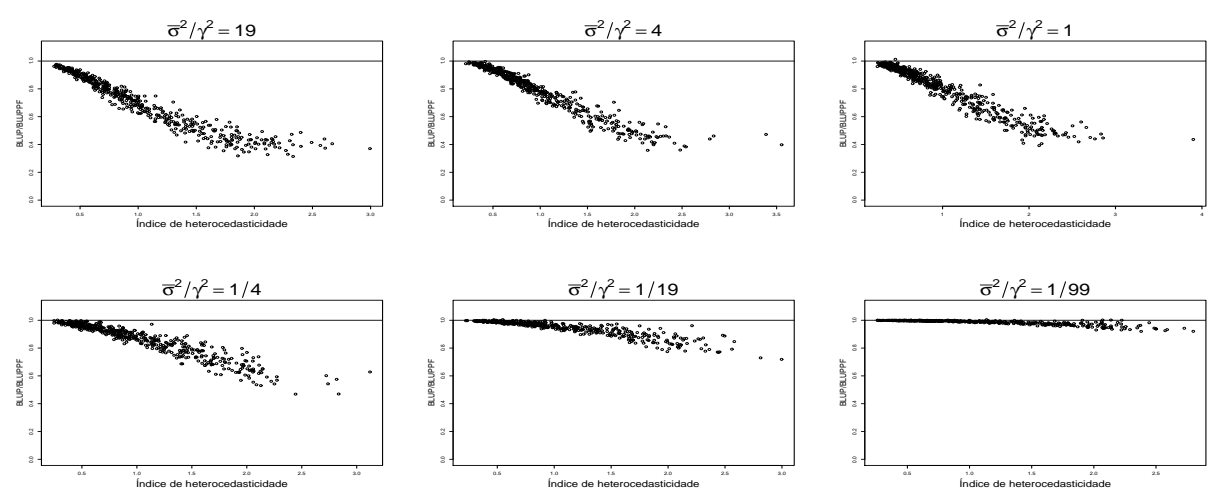

Y's gerados com distribuição assimétrica à esquerda
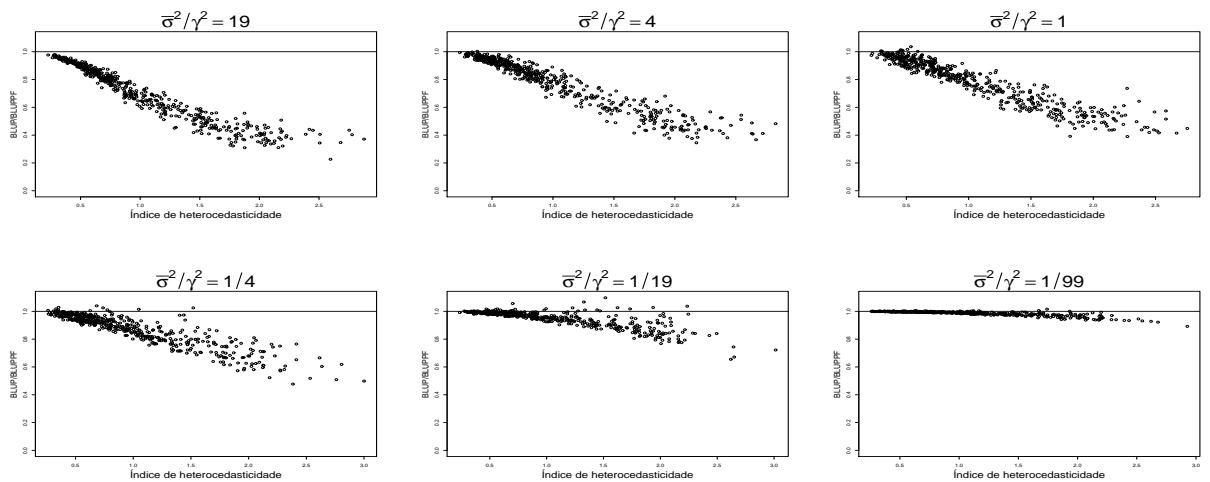

Y's gerados com distribuição assimétrica à direita
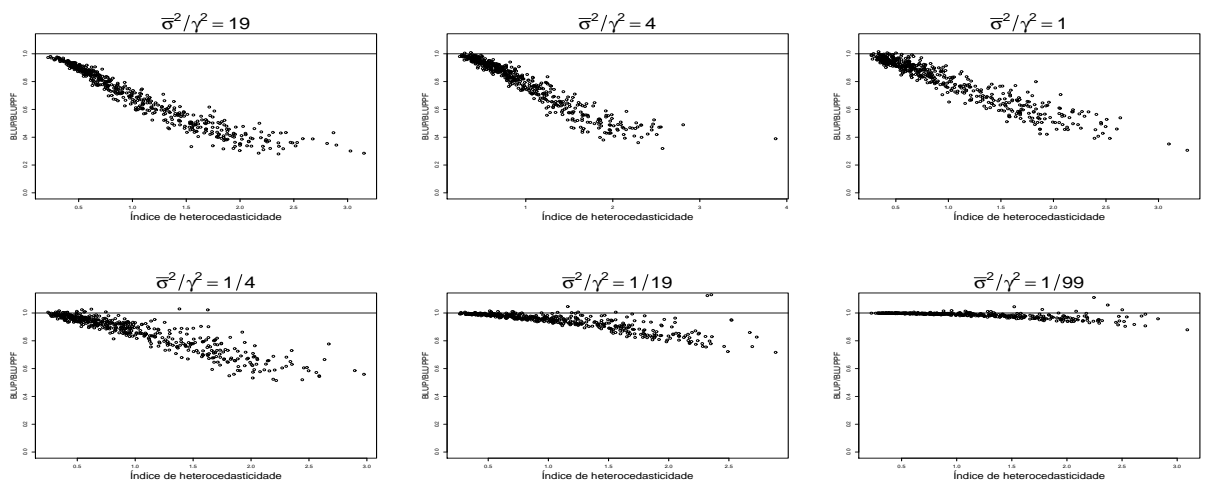
Figura H.6: Quocientes EQM $\left[\hat{Q}_{i}^{(1)}\right] / \operatorname{EMQ}\left[\hat{Q}_{i}^{(2)}\right]$ sob erros de medida endógenos com distribuição beta assimétrica, $N=100$ e $n=50$.

Y's gerados com distribuição uniforme
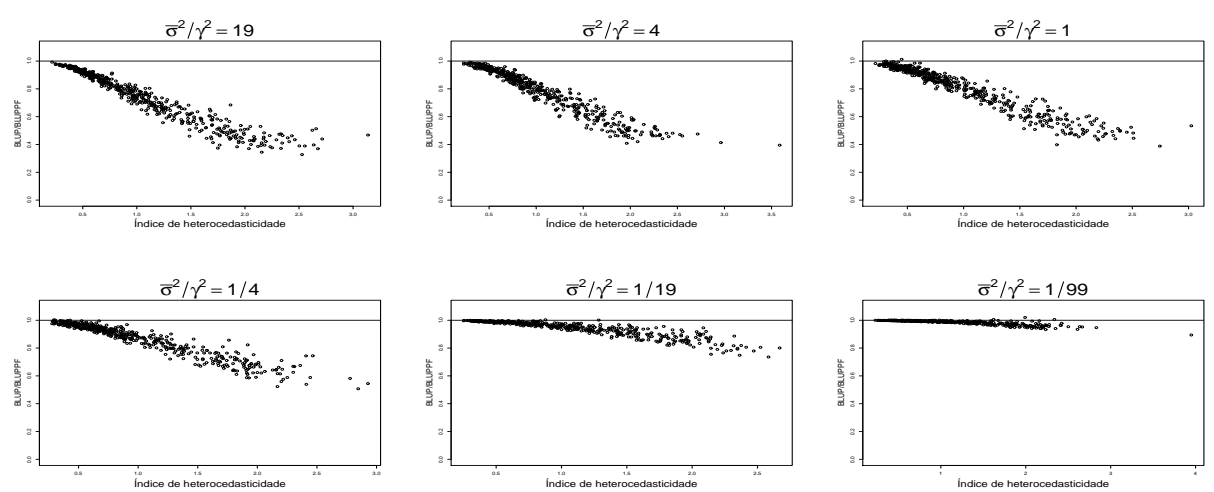

Y's gerados com distribuição assimétrica à esquerda
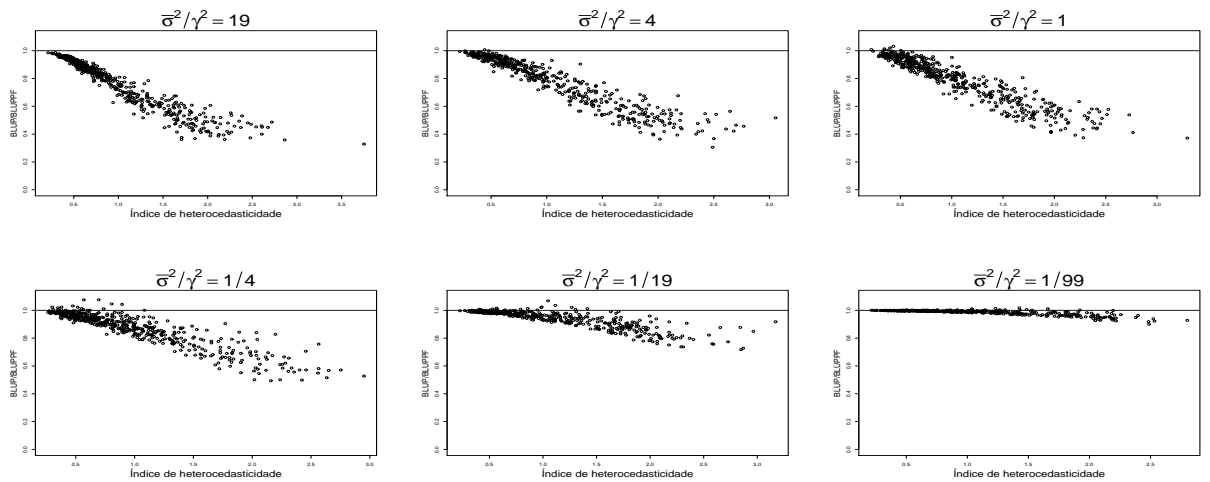

Y's gerados com distribuição assimétrica à direita
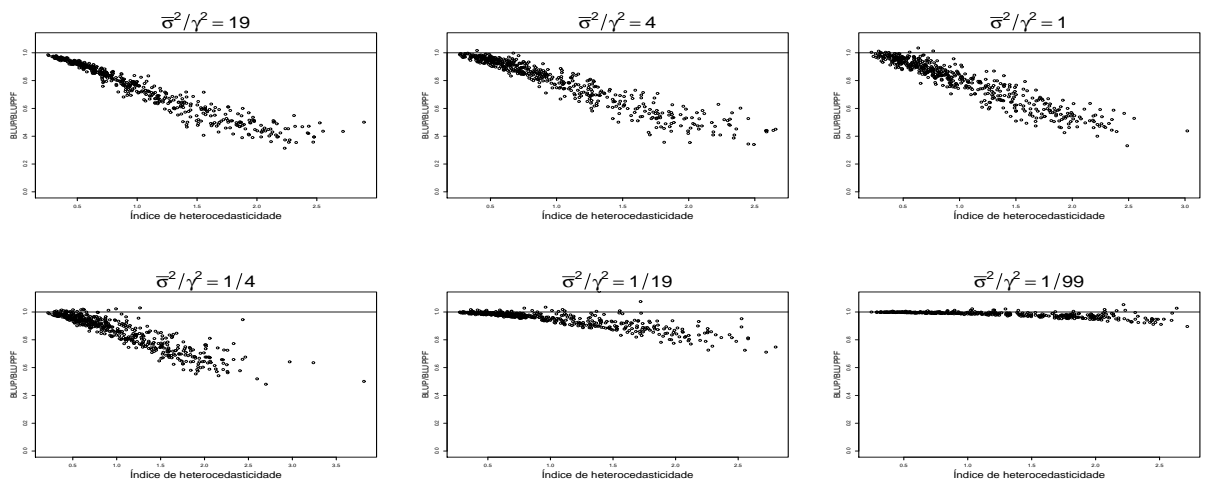
Figura H.7: Quocientes EQM $\left[\hat{Q}_{i}^{(1)}\right] / \operatorname{EMQ}\left[\hat{Q}_{i}^{(2)}\right]$ sob erros de medida endógenos com distribuição beta assimétrica, $N=300$ e $n=30$.

Y's gerados com distribuição uniforme
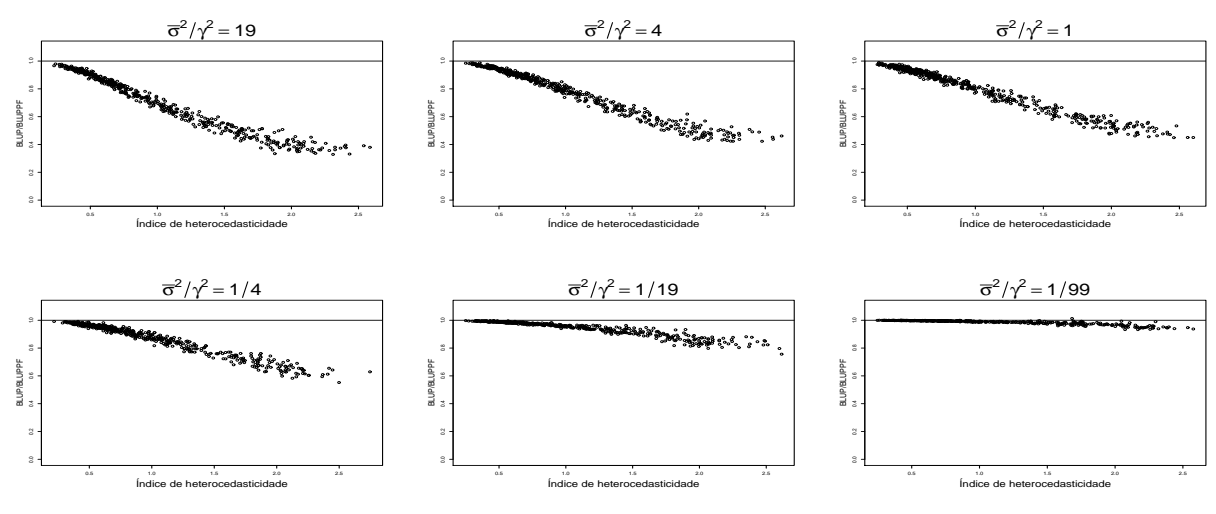

Y's gerados com distribuição assimétrica à esquerda
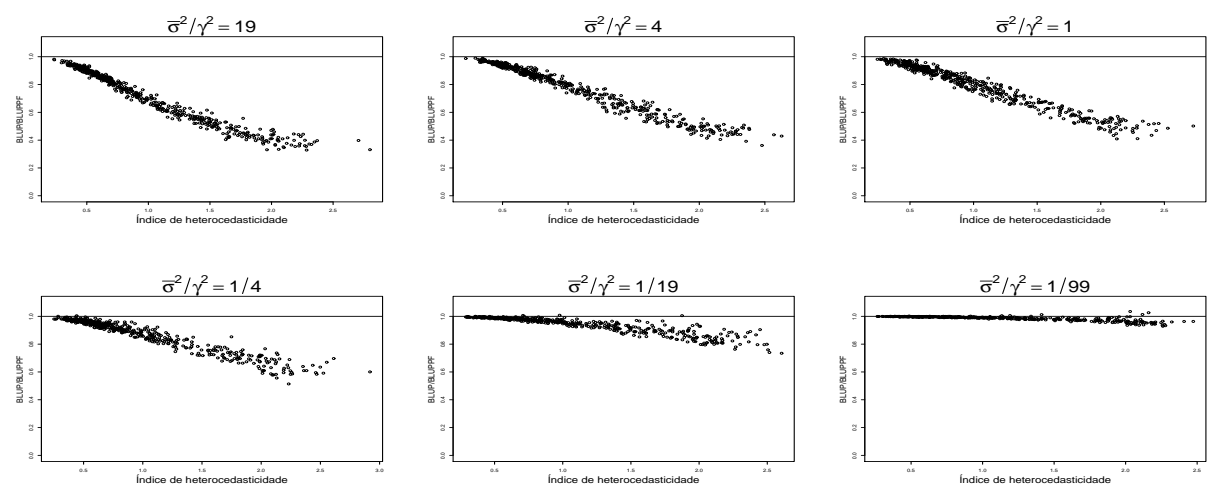

Y's gerados com distribuição assimétrica à direita
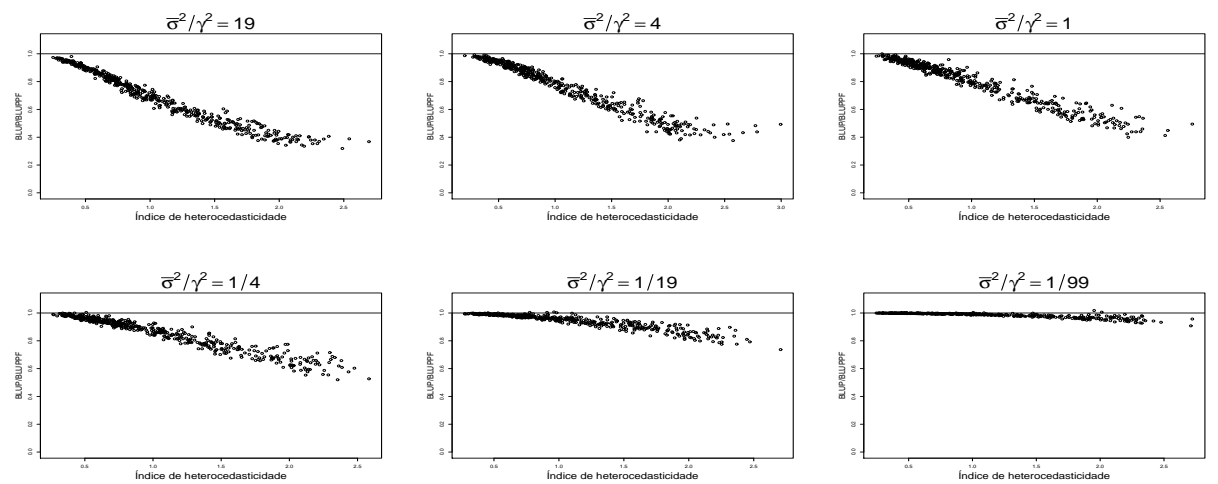
Figura H.8: Quocientes EQM $\left[\hat{Q}_{i}^{(1)}\right] / \operatorname{EMQ}\left[\hat{Q}_{i}^{(2)}\right]$ sob erros de medida endógenos com distribuição beta assimétrica, $N=300$ e $n=50$.

Y's gerados com distribuição uniforme
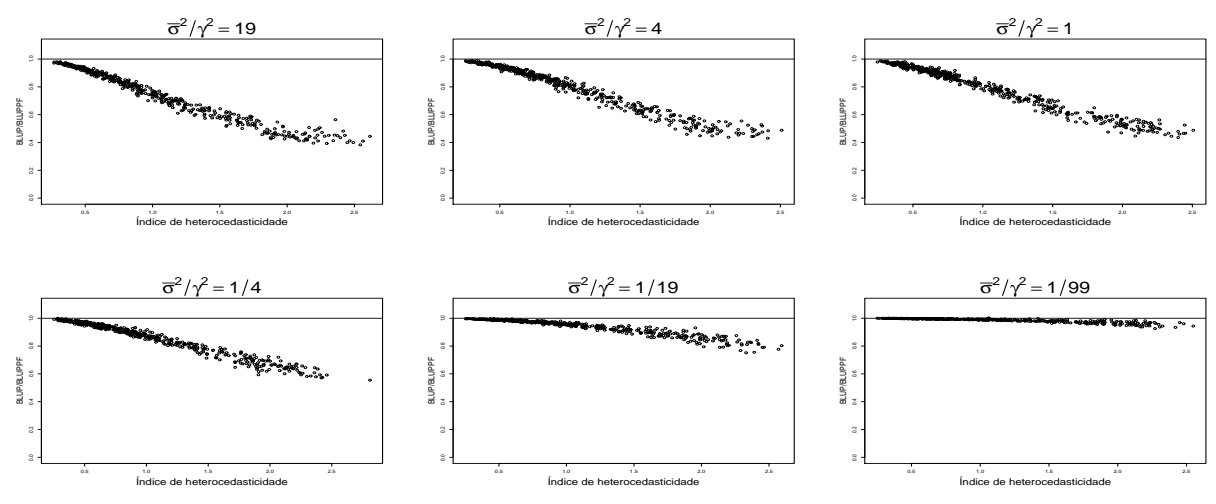

Y's gerados com distribuição assimétrica à esquerda
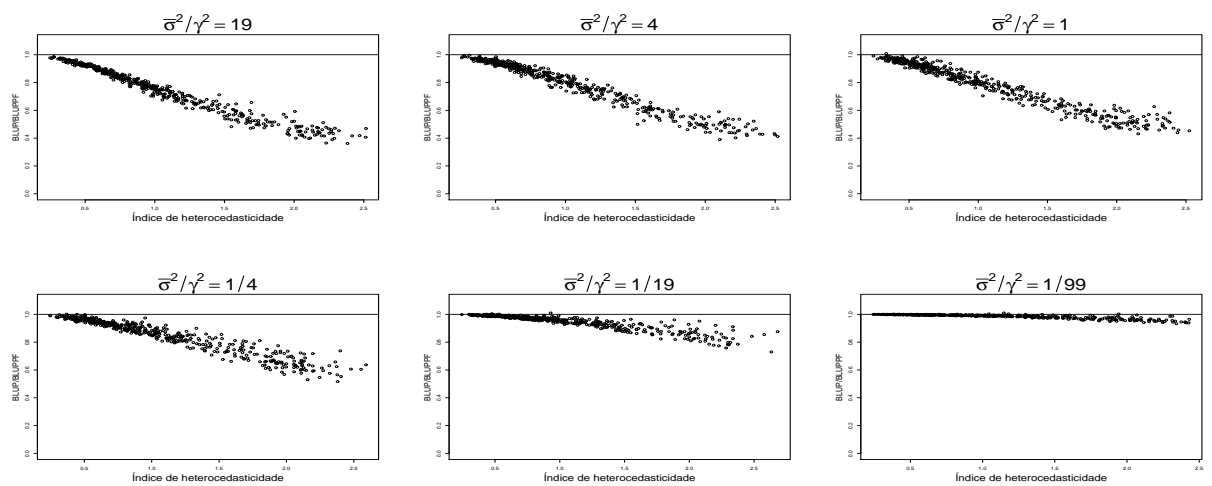

Y's gerados com distribuição assimétrica à direita
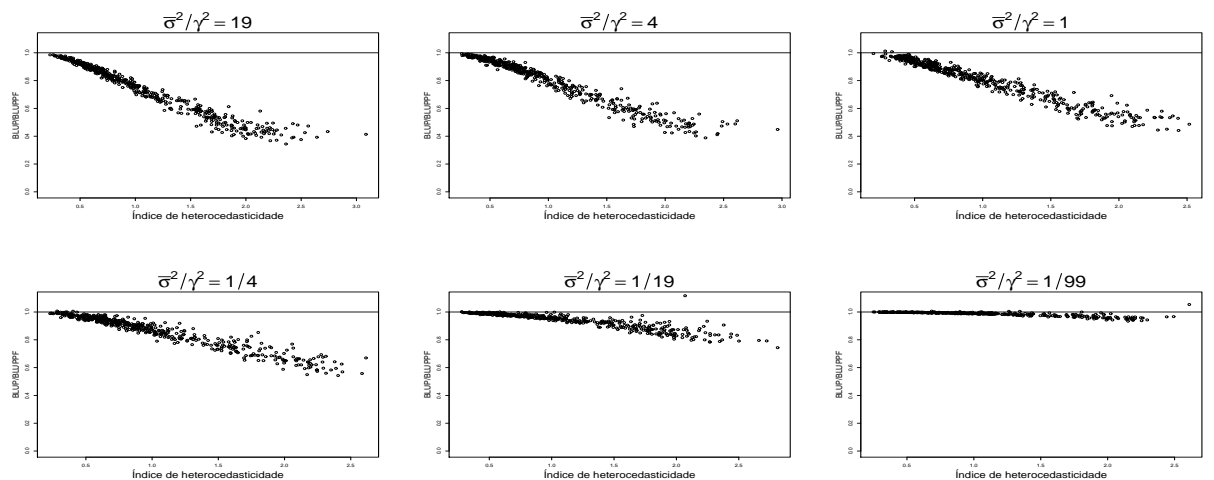
Figura H.9: Quocientes EQM $\left[\hat{Q}_{i}^{(1)}\right] / \operatorname{EMQ}\left[\hat{Q}_{i}^{(2)}\right]$ sob erros de medida endógenos com distribuição beta assimétrica, $N=300$ e $n=100$.

Y's gerados com distribuição uniforme
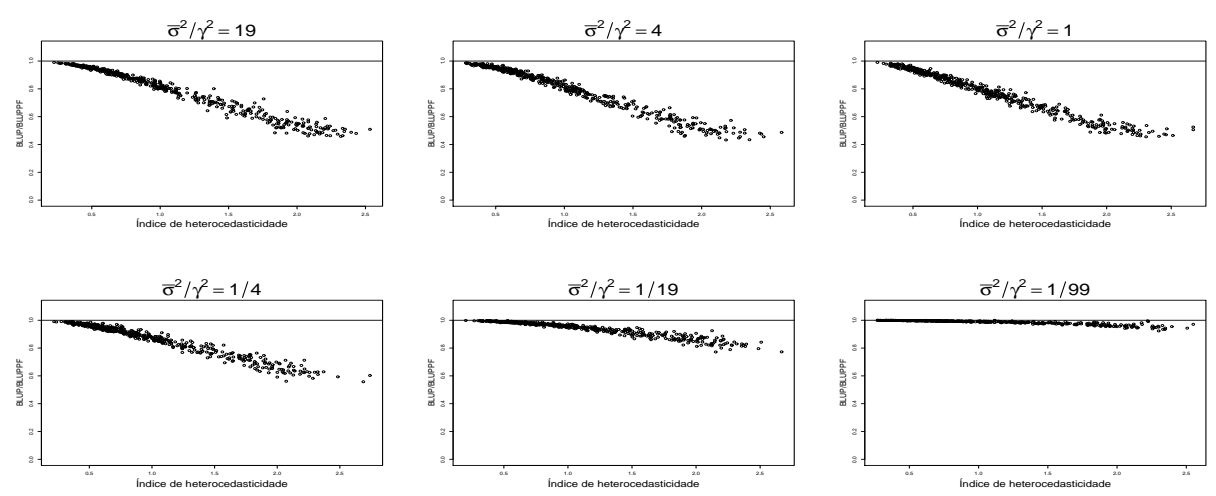

Y's gerados com distribuição assimétrica à esquerda
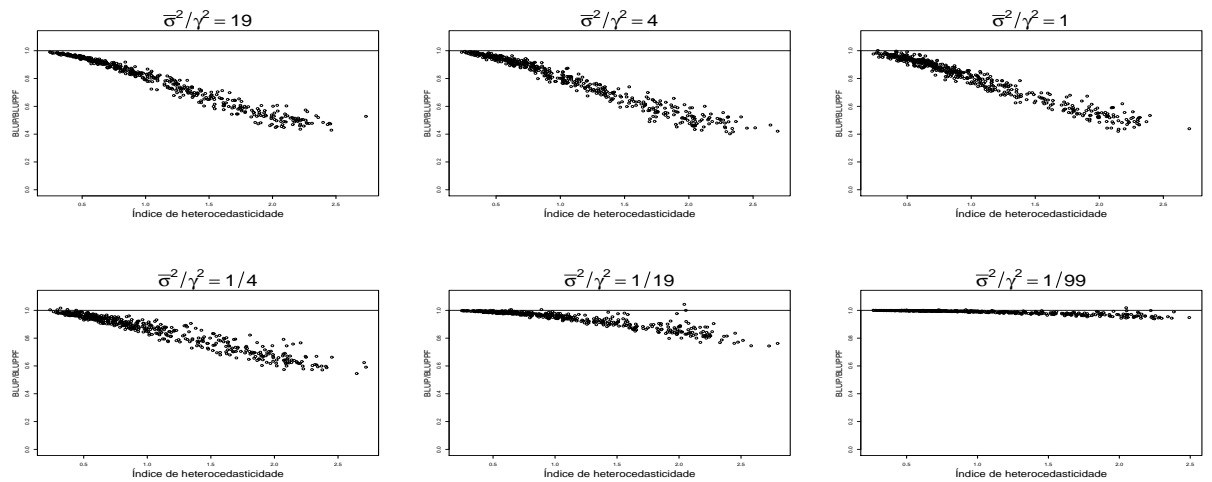

Y's gerados com distribuição assimétrica à direita
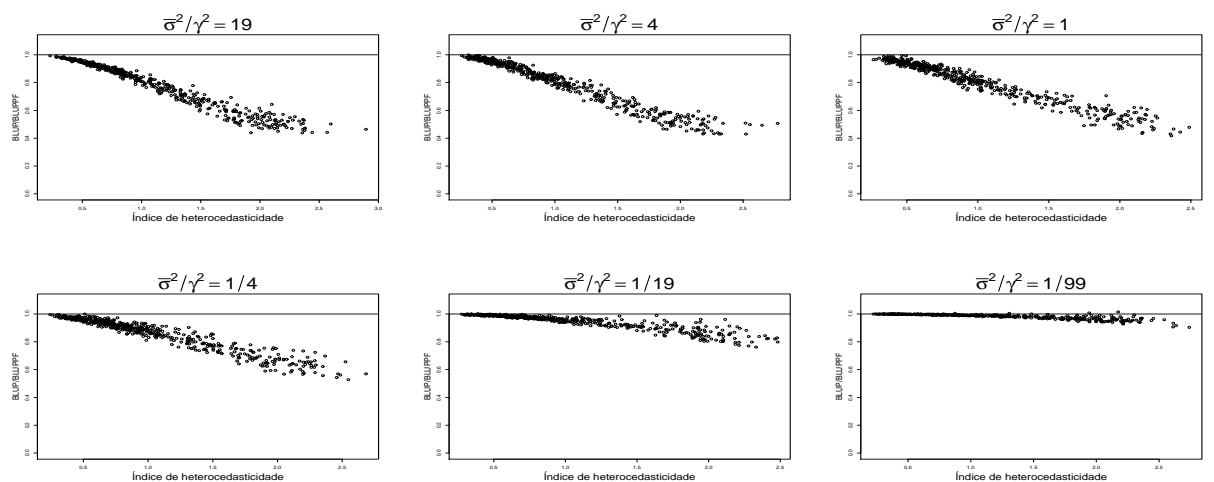
Apêndice I

Quocientes EQM $\left[\hat{Q}_{i}^{(1)}\right] / \operatorname{EMQ}\left[\hat{Q}_{i}^{(2)}\right]$ sob erros de medida endógenos com distribuição exponencial 
Figura I.1: Quocientes $\operatorname{EQM}\left[\hat{Q}_{i}^{(1)}\right] / \operatorname{EMQ}\left[\hat{Q}_{i}^{(2)}\right]$ sob erros de medida endógenos com distribuição exponencial, $N=50$ e $n=5$.

Y's gerados com distribuição uniforme
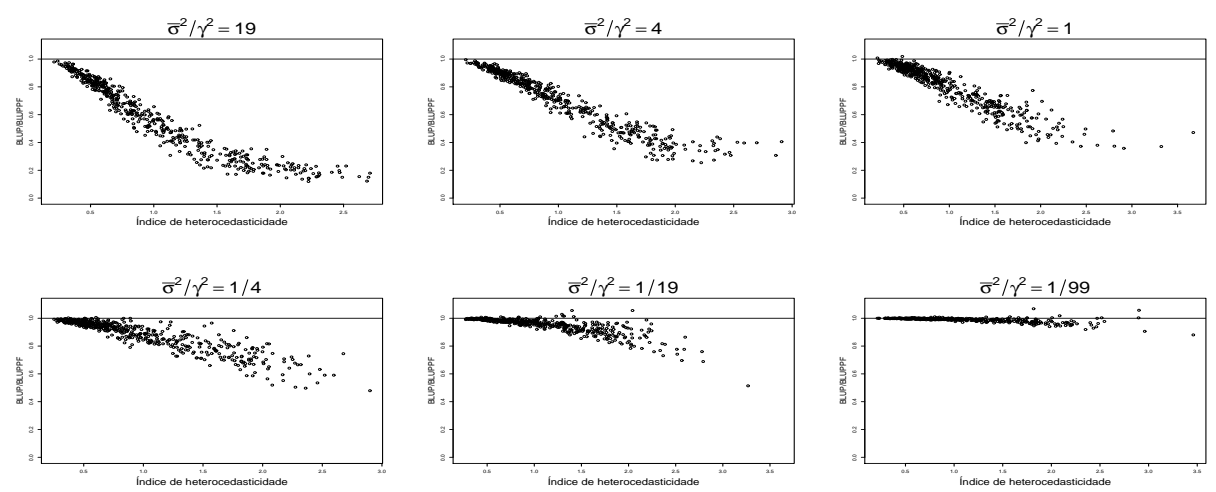

Y's gerados com distribuição assimétrica à esquerda
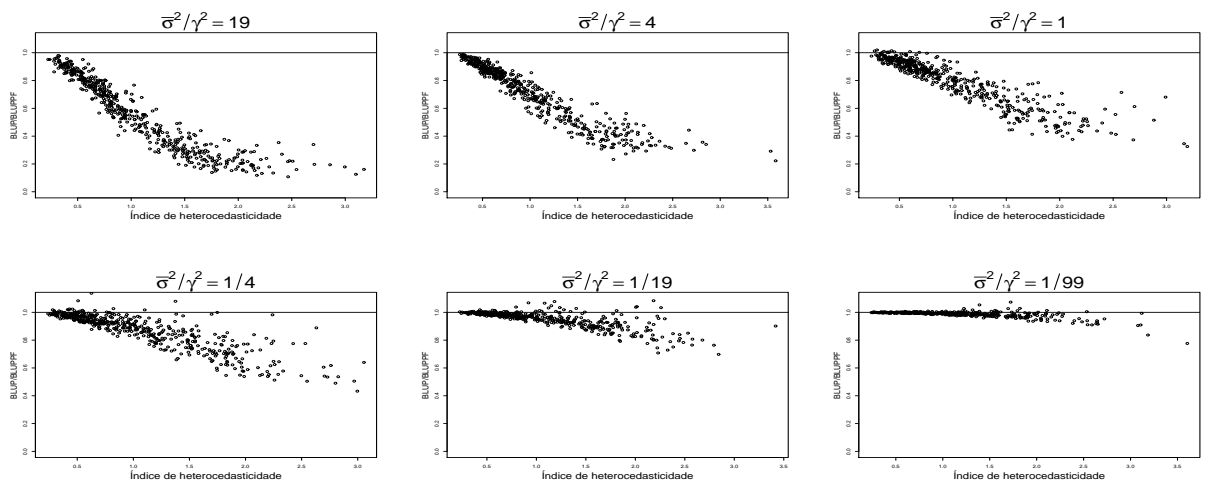

Y's gerados com distribuição assimétrica à direita
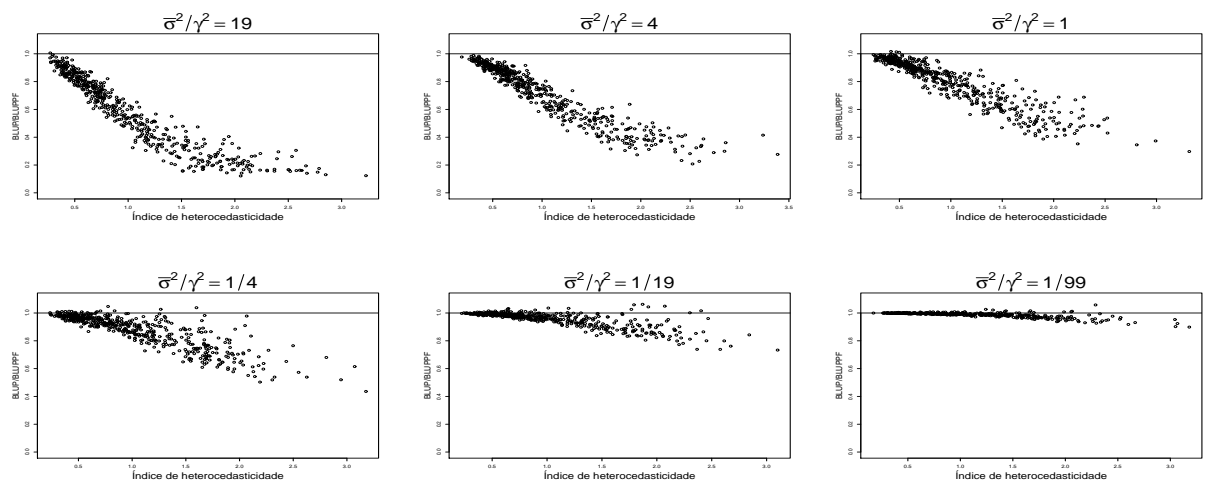
Figura I.2: Quocientes $\operatorname{EQM}\left[\hat{Q}_{i}^{(1)}\right] / \operatorname{EMQ}\left[\hat{Q}_{i}^{(2)}\right]$ sob erros de medida endógenos com distribuição exponencial, $N=50$ e $n=10$.

Y's gerados com distribuição uniforme
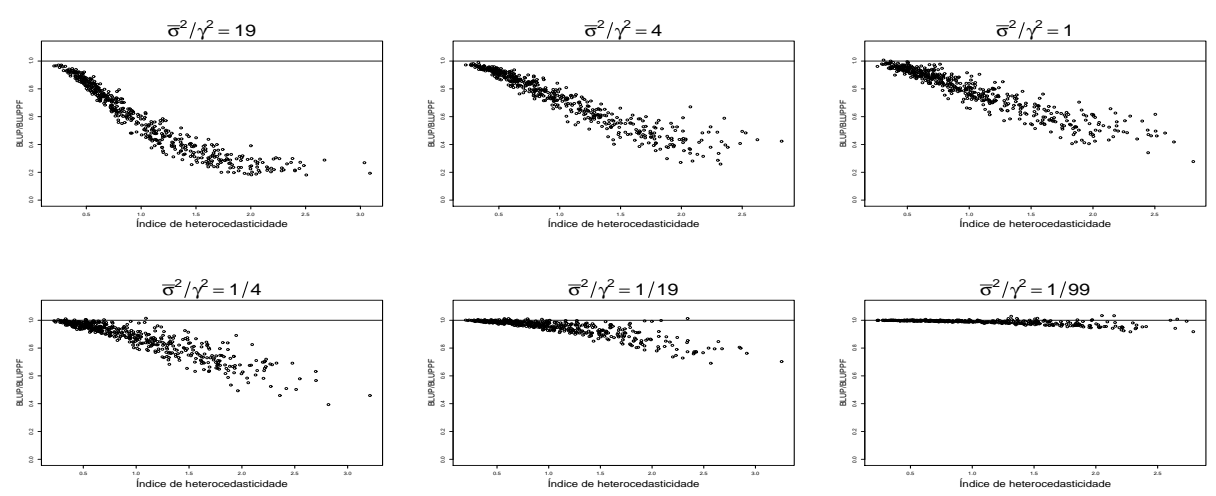

Y's gerados com distribuição assimétrica à esquerda
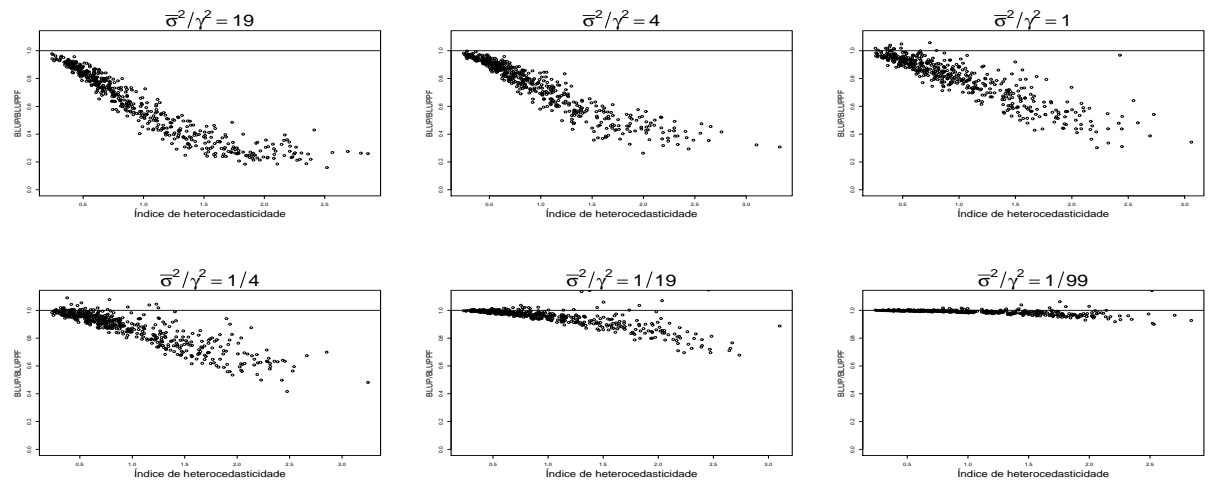

Y's gerados com distribuição assimétrica à direita
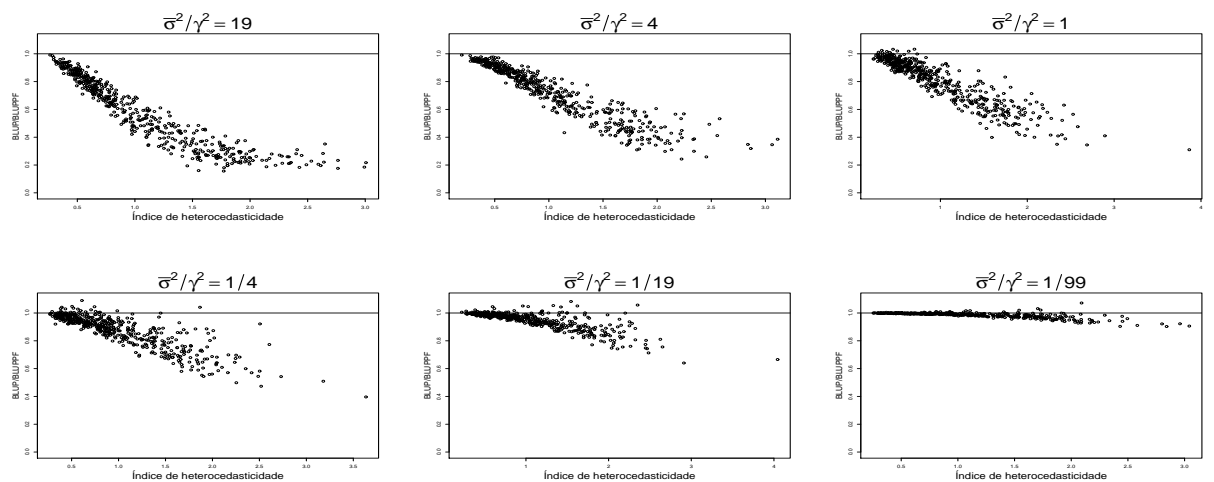
Figura I.3: Quocientes $\operatorname{EQM}\left[\hat{Q}_{i}^{(1)}\right] / \operatorname{EMQ}\left[\hat{Q}_{i}^{(2)}\right]$ sob erros de medida endógenos com distribuição exponencial, $N=50$ e $n=25$.

Y's gerados com distribuição uniforme
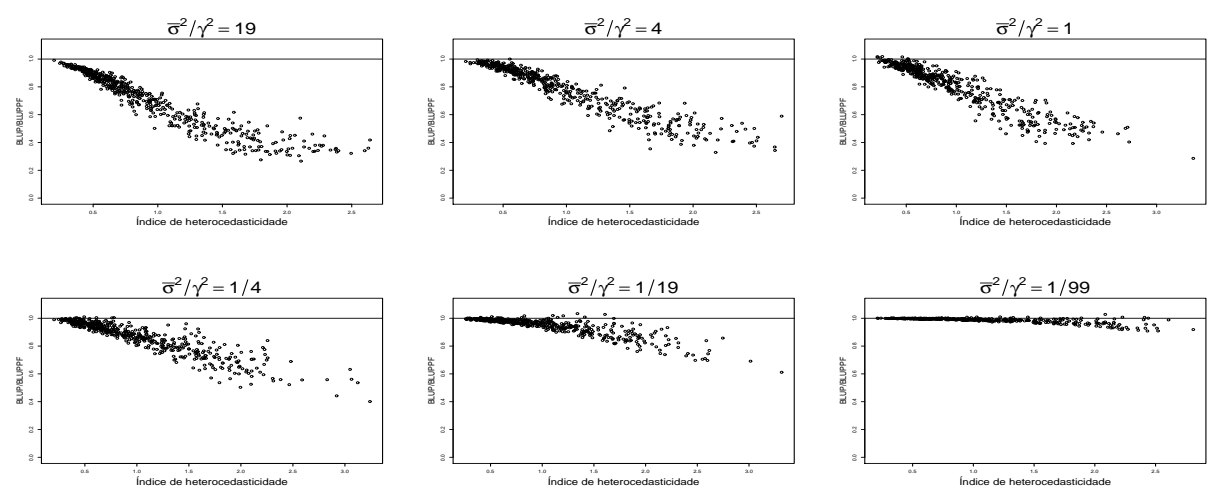

Y's gerados com distribuição assimétrica à esquerda
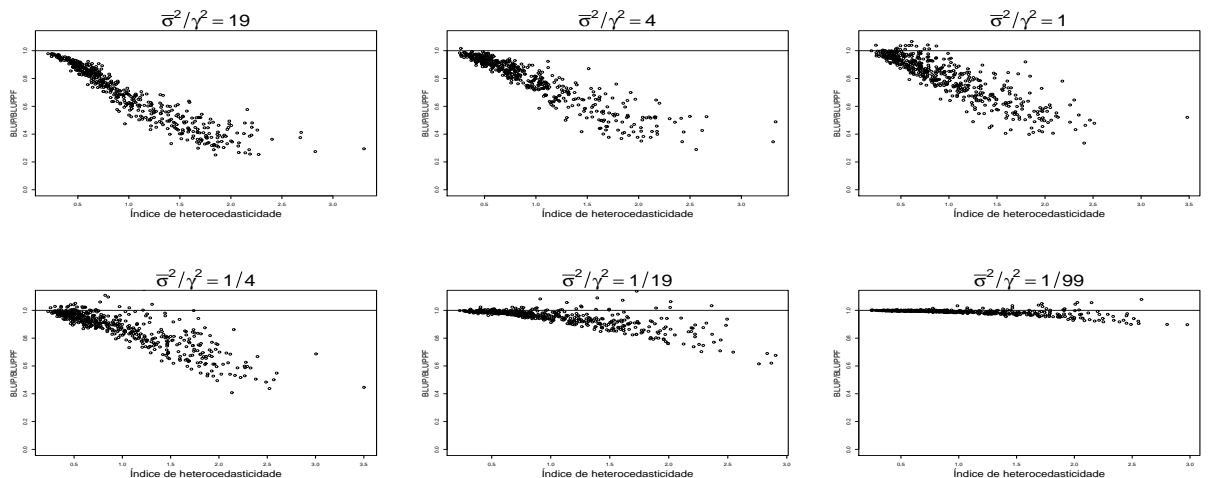

Y's gerados com distribuição assimétrica à direita
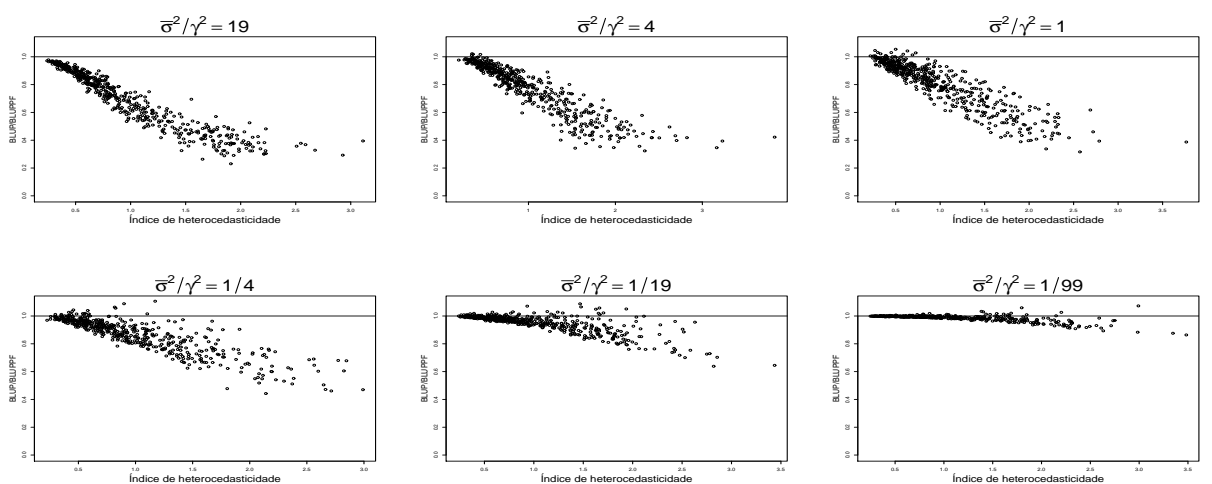
Figura I.4: Quocientes $\operatorname{EQM}\left[\hat{Q}_{i}^{(1)}\right] / \operatorname{EMQ}\left[\hat{Q}_{i}^{(2)}\right]$ sob erros de medida endógenos com distribuição exponencial, $N=100$ e $n=10$.

Y's gerados com distribuição uniforme
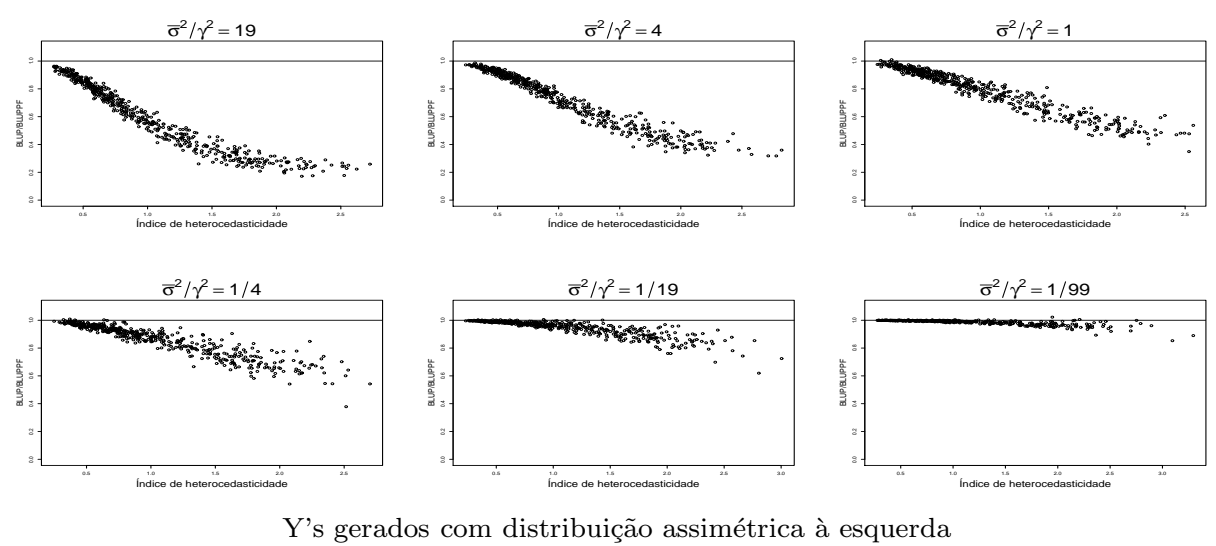

Y's gerados com distribuição assimétrica à esquerda
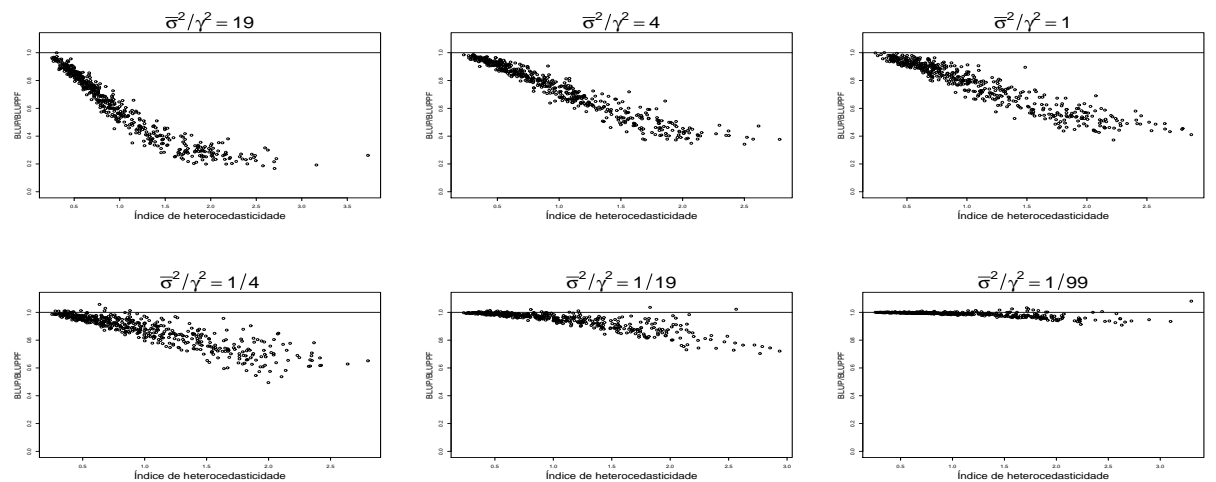

Y's gerados com distribuição assimétrica à direita
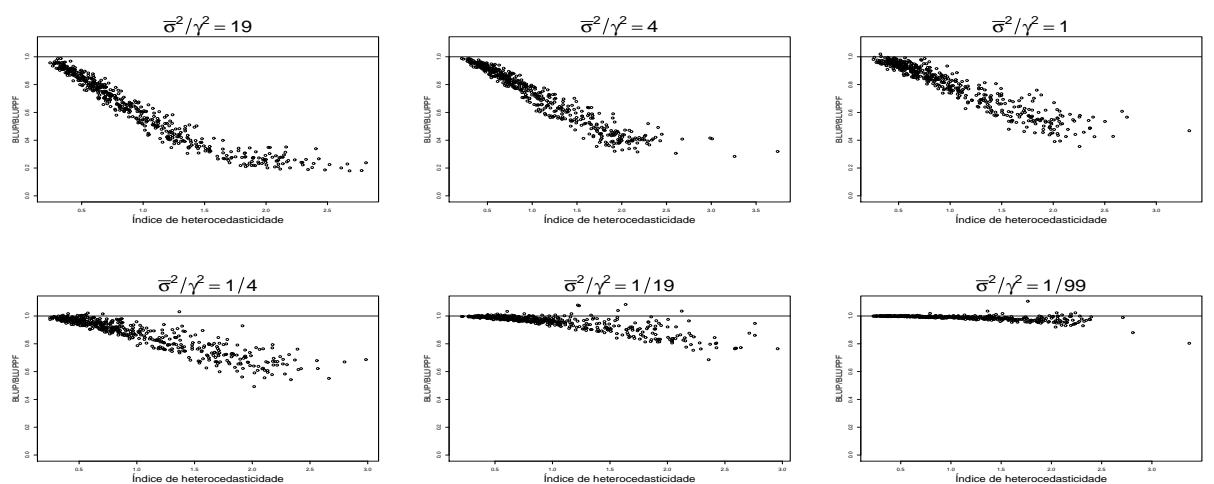
Figura I.5: Quocientes $\operatorname{EQM}\left[\hat{Q}_{i}^{(1)}\right] / \operatorname{EMQ}\left[\hat{Q}_{i}^{(2)}\right]$ sob erros de medida endógenos com distribuição exponencial, $N=100$ e $n=30$.

Y's gerados com distribuição uniforme
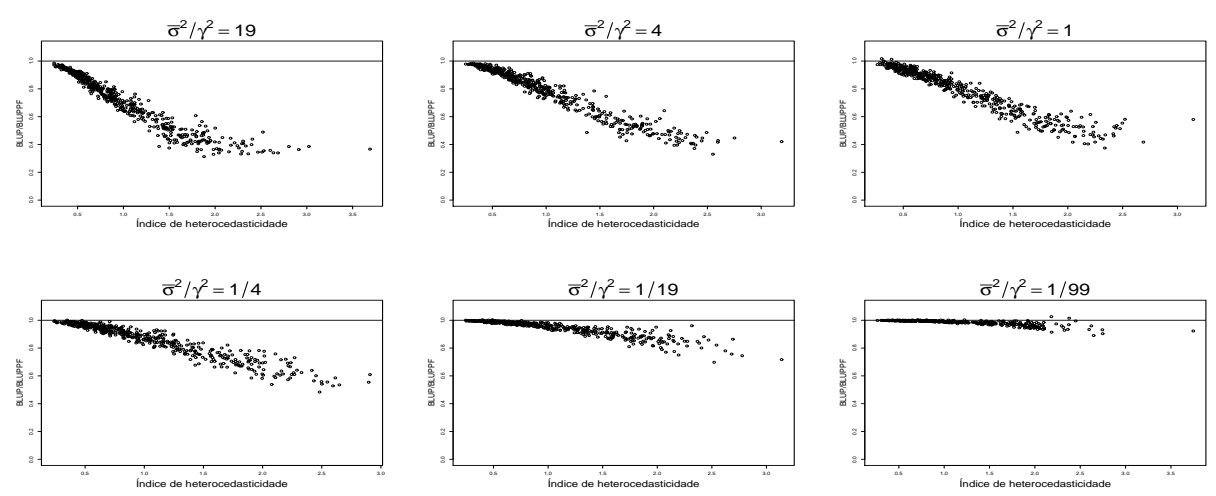

Y's gerados com distribuição assimétrica à esquerda
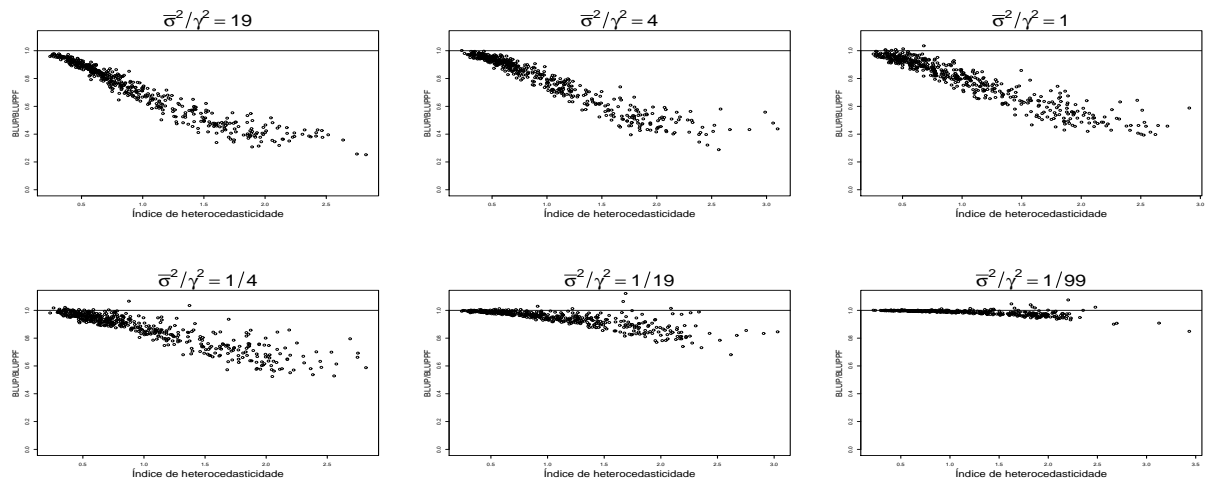

Y's gerados com distribuição assimétrica à direita
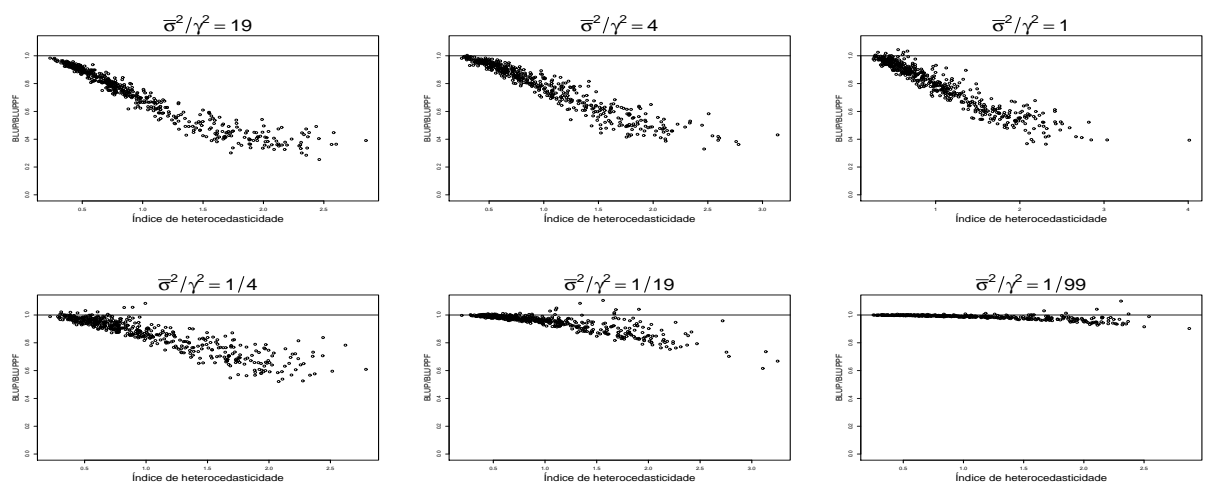
Figura I.6: Quocientes $\operatorname{EQM}\left[\hat{Q}_{i}^{(1)}\right] / \operatorname{EMQ}\left[\hat{Q}_{i}^{(2)}\right]$ sob erros de medida endógenos com distribuição exponencial, $N=100$ e $n=50$.

Y's gerados com distribuição uniforme
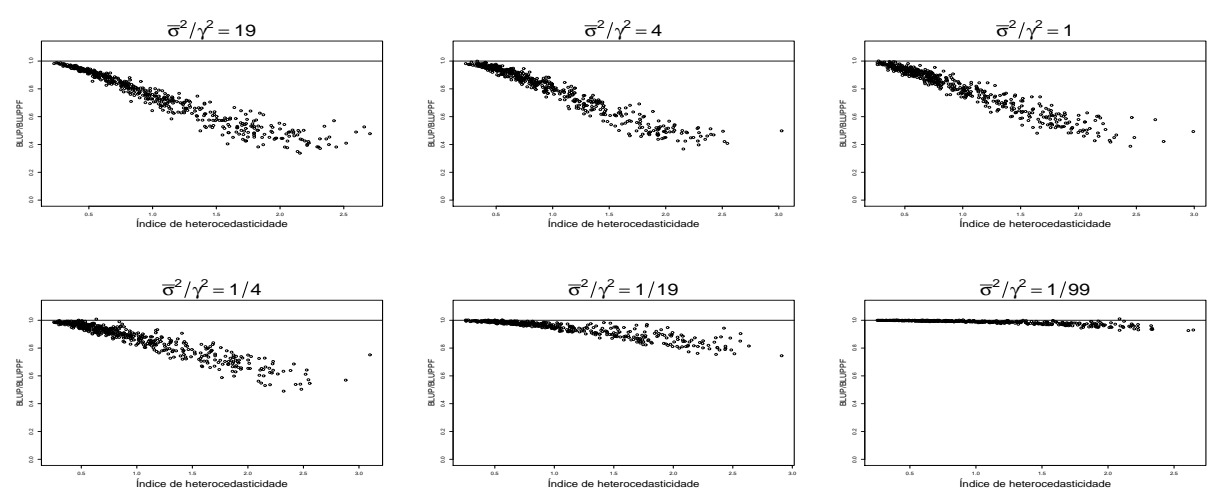

Y's gerados com distribuição assimétrica à esquerda
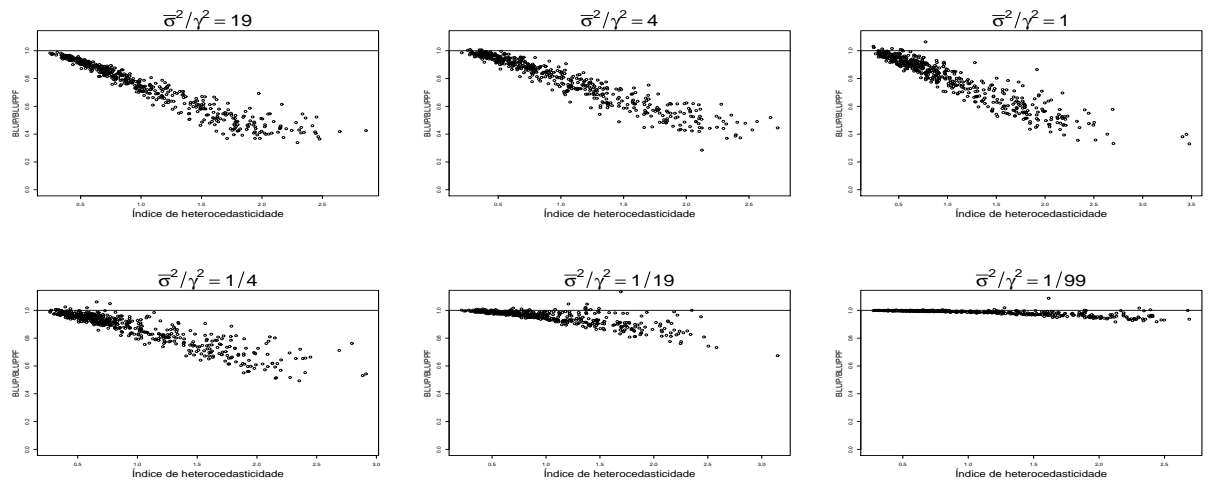

Y's gerados com distribuição assimétrica à direita
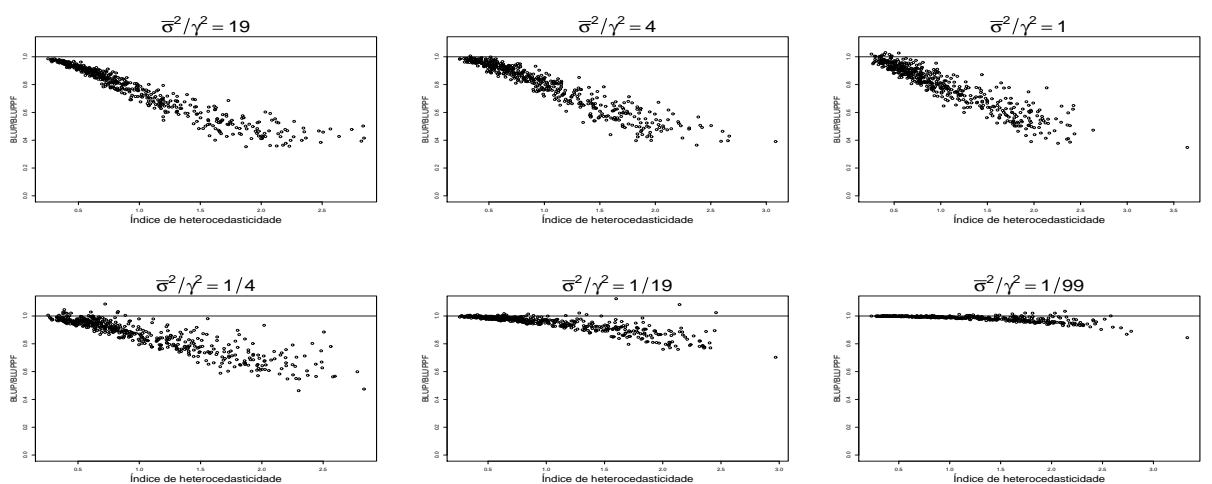
Figura I.7: Quocientes $\operatorname{EQM}\left[\hat{Q}_{i}^{(1)}\right] / \operatorname{EMQ}\left[\hat{Q}_{i}^{(2)}\right]$ sob erros de medida endógenos com distribuição exponencial, $N=300$ e $n=30$.

Y's gerados com distribuição uniforme
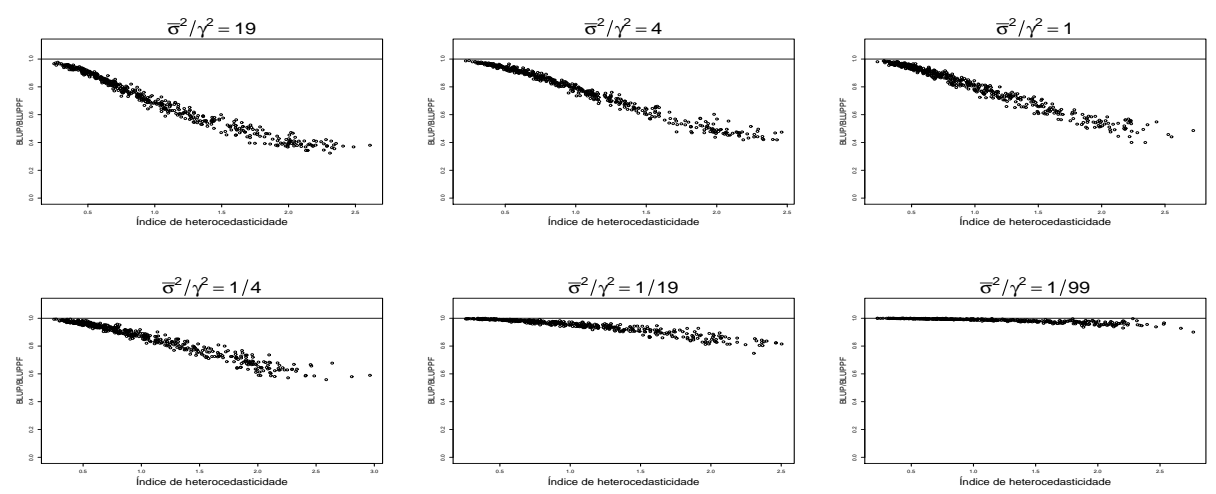

Y's gerados com distribuição assimétrica à esquerda
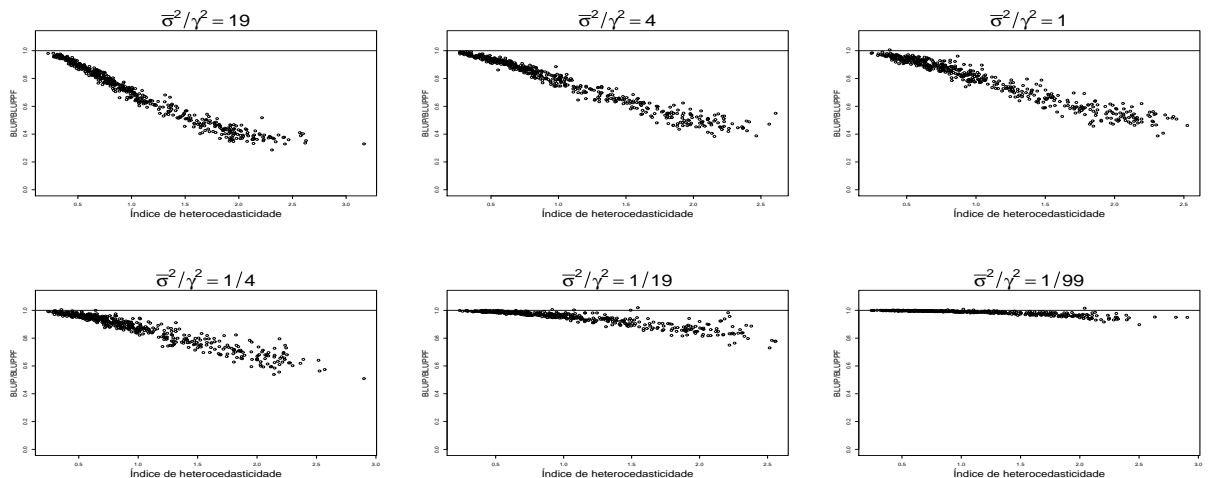

Y's gerados com distribuição assimétrica à direita
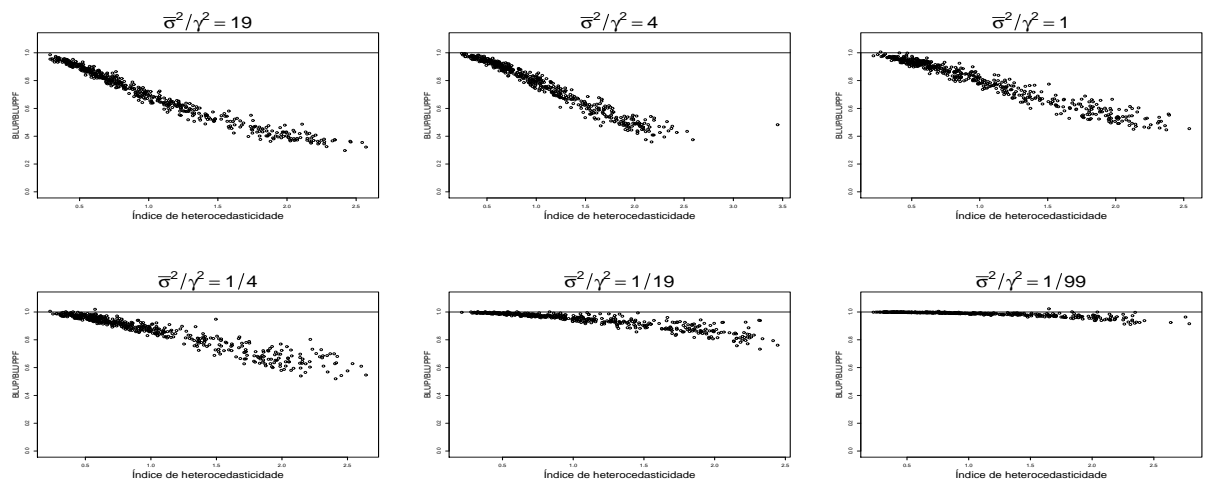
Figura I.8: Quocientes $\operatorname{EQM}\left[\hat{Q}_{i}^{(1)}\right] / \operatorname{EMQ}\left[\hat{Q}_{i}^{(2)}\right]$ sob erros de medida endógenos com distribuição exponencial, $N=300$ e $n=50$.

Y's gerados com distribuição uniforme
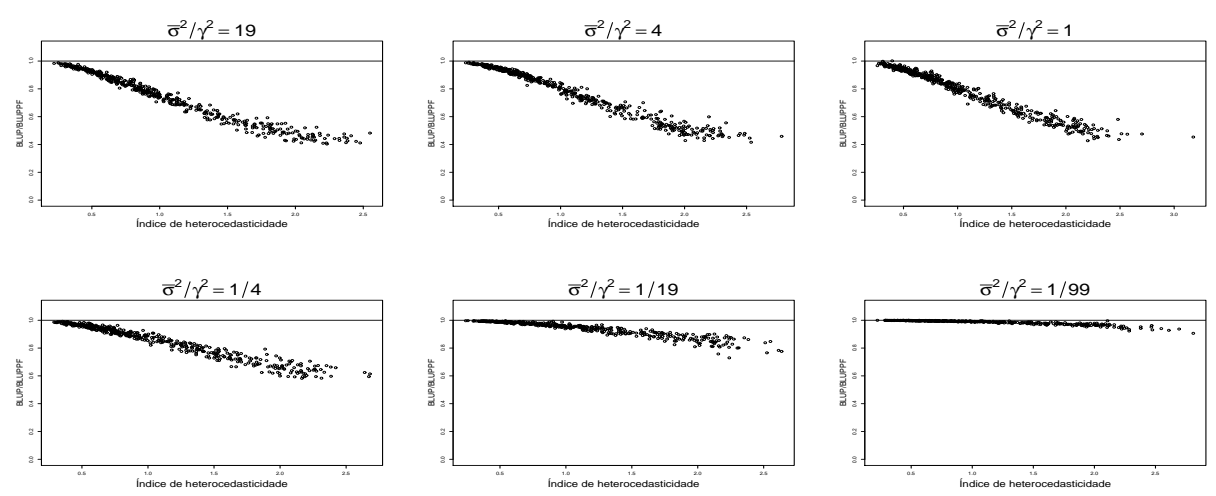

Y's gerados com distribuição assimétrica à esquerda
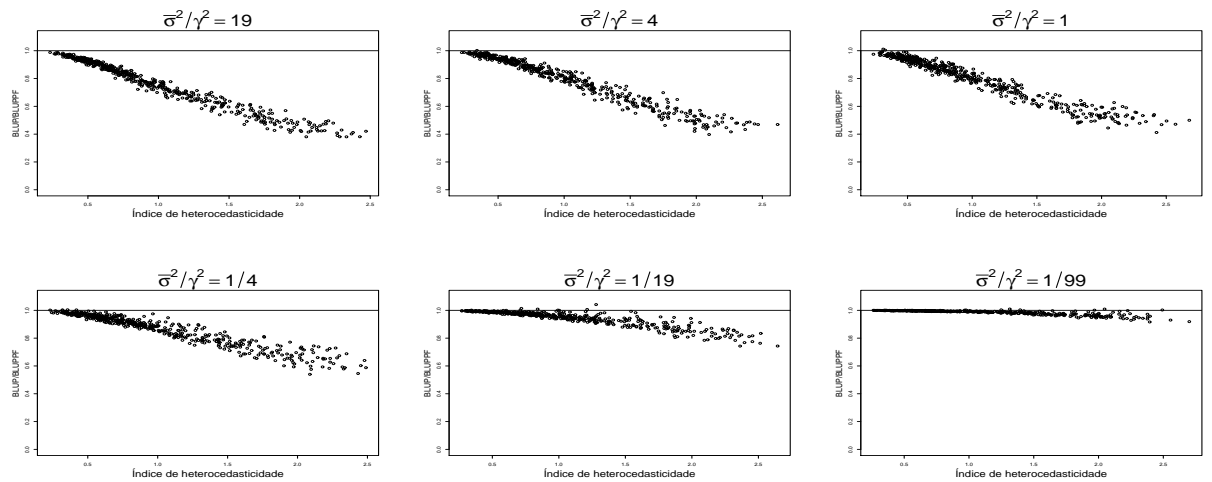

Y's gerados com distribuição assimétrica à direita
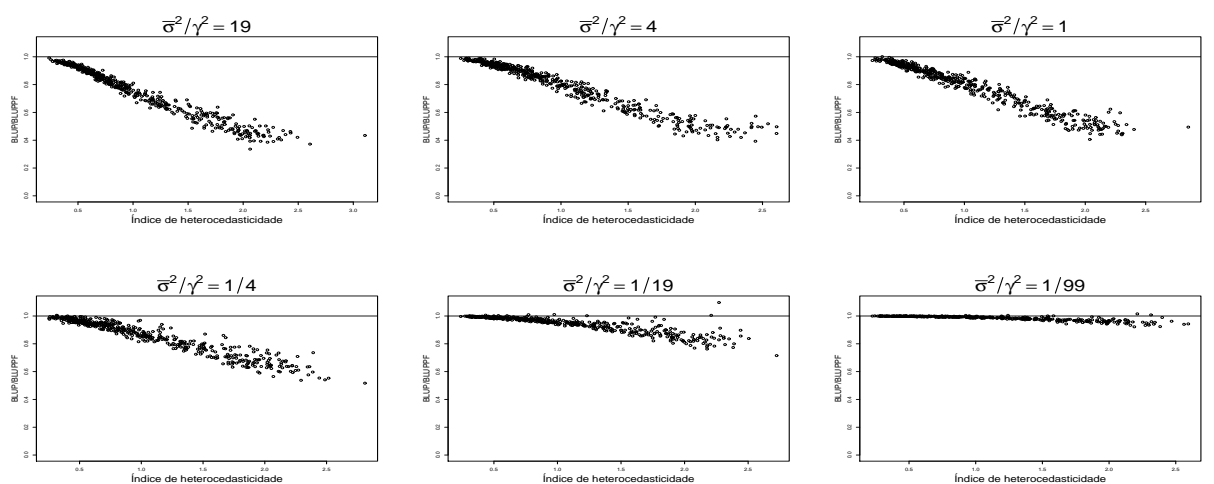
Figura I.9: Quocientes $\operatorname{EQM}\left[\hat{Q}_{i}^{(1)}\right] / \operatorname{EMQ}\left[\hat{Q}_{i}^{(2)}\right]$ sob erros de medida endógenos com distribuição exponencial, $N=300$ e $n=100$.

Y's gerados com distribuição uniforme
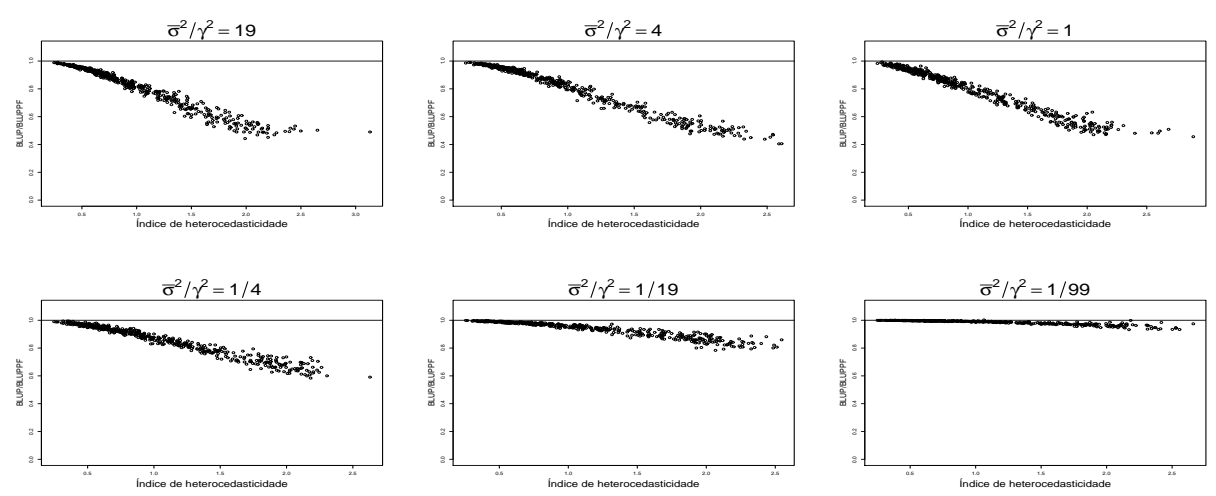

Y's gerados com distribuição assimétrica à esquerda
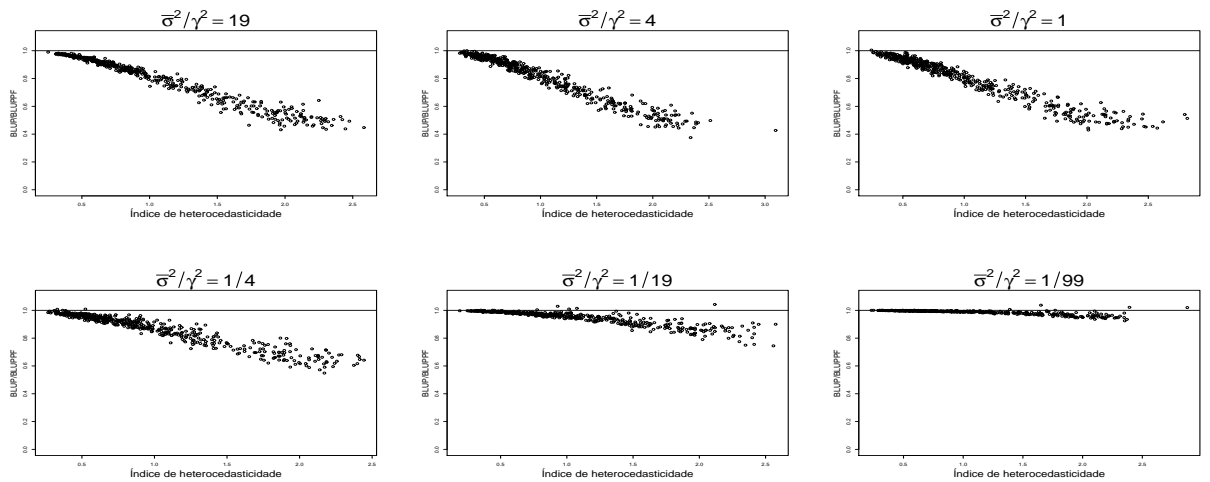

Y's gerados com distribuição assimétrica à direita
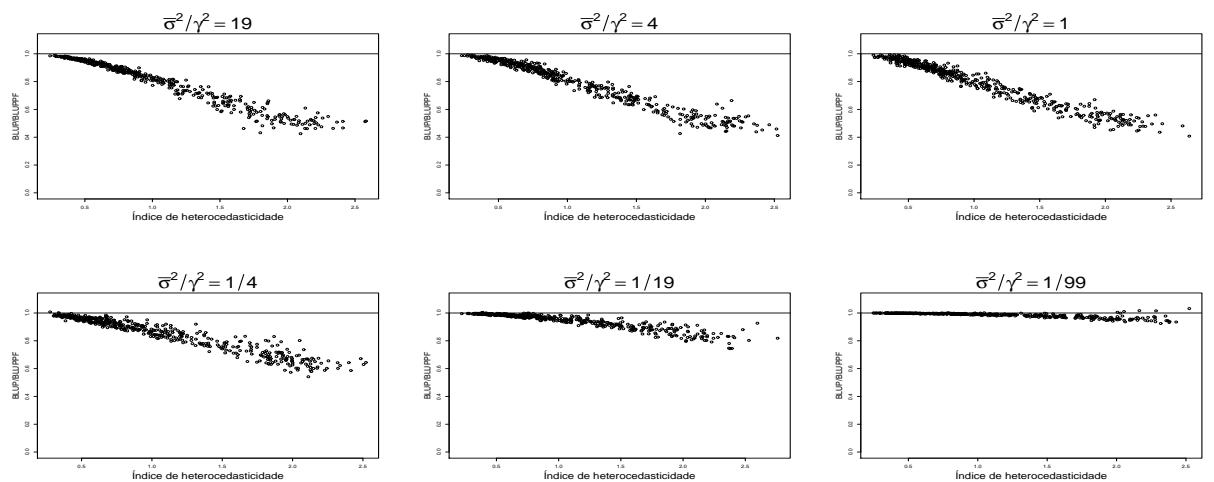
Apêndice J

Quocientes EQM $\left[\hat{Q}_{i}^{(1)}\right] / \operatorname{EMQ}\left[\hat{Q}_{i}^{(2)}\right]$ sob erros de medida endógenos com distribuição gama 
Figura J.1: Quocientes $\operatorname{EQM}\left[\hat{Q}_{i}^{(1)}\right] / \operatorname{EMQ}\left[\hat{Q}_{i}^{(2)}\right]$ sob erros de medida endógenos com distribuição gama, $N=50$ e $n=5$.

Y's gerados com distribuição uniforme
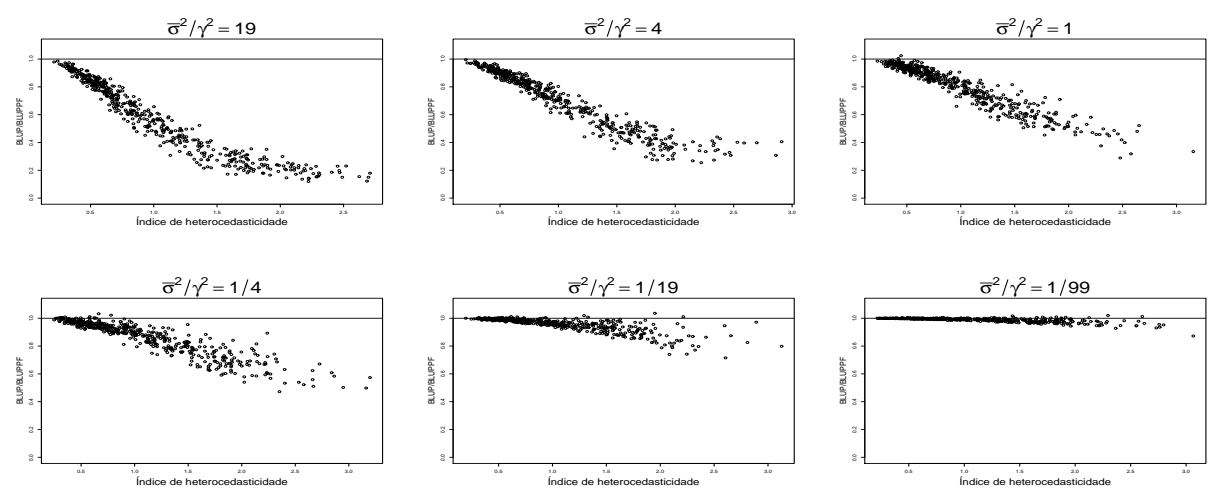

Y's gerados com distribuição assimétrica à esquerda
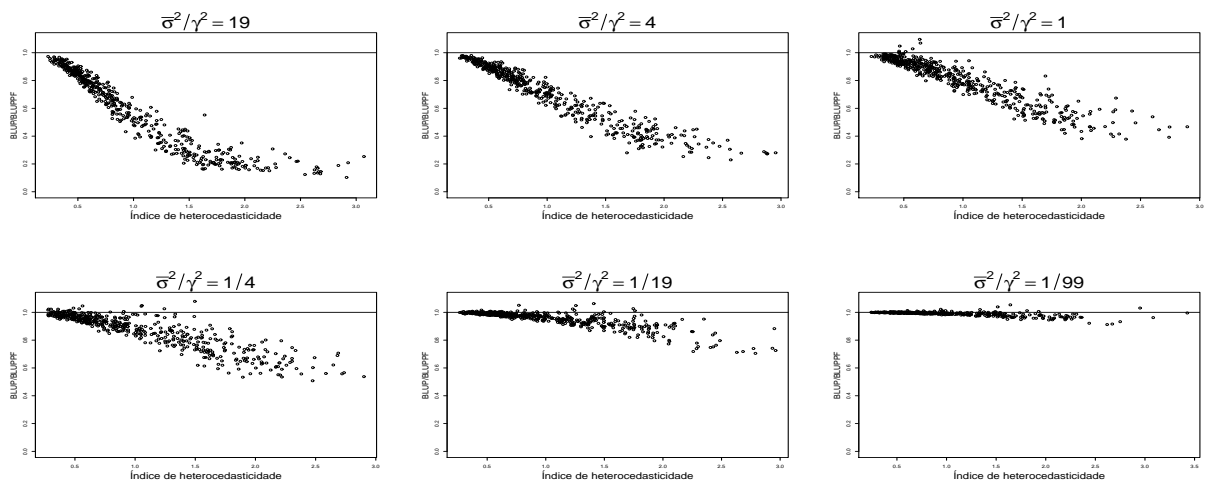

Y's gerados com distribuição assimétrica à direita
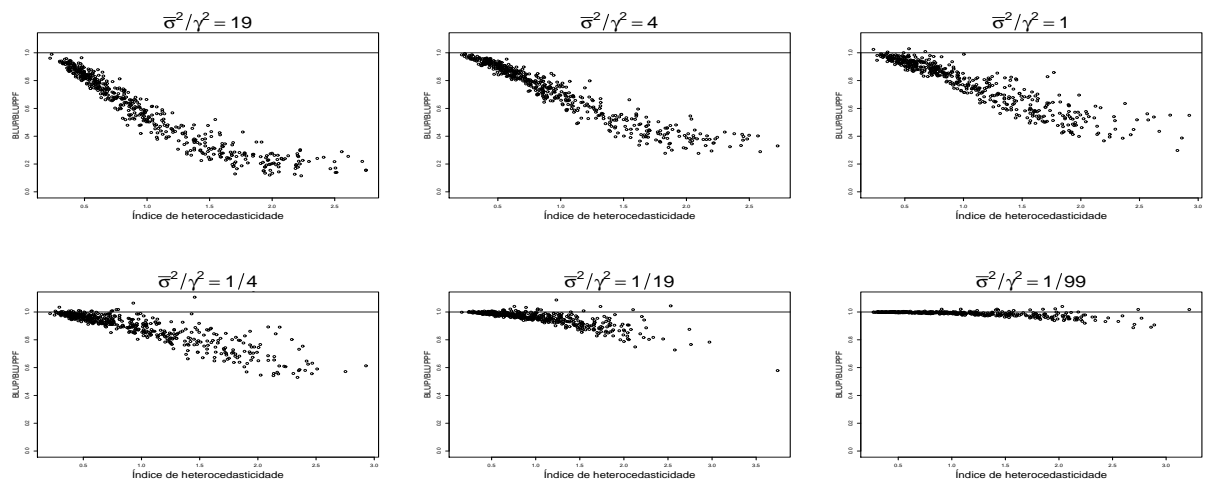
Figura J.2: Quocientes $\operatorname{EQM}\left[\hat{Q}_{i}^{(1)}\right] / \operatorname{EMQ}\left[\hat{Q}_{i}^{(2)}\right]$ sob erros de medida endógenos com distribuição gama, $N=50$ e $n=10$.

Y's gerados com distribuição uniforme
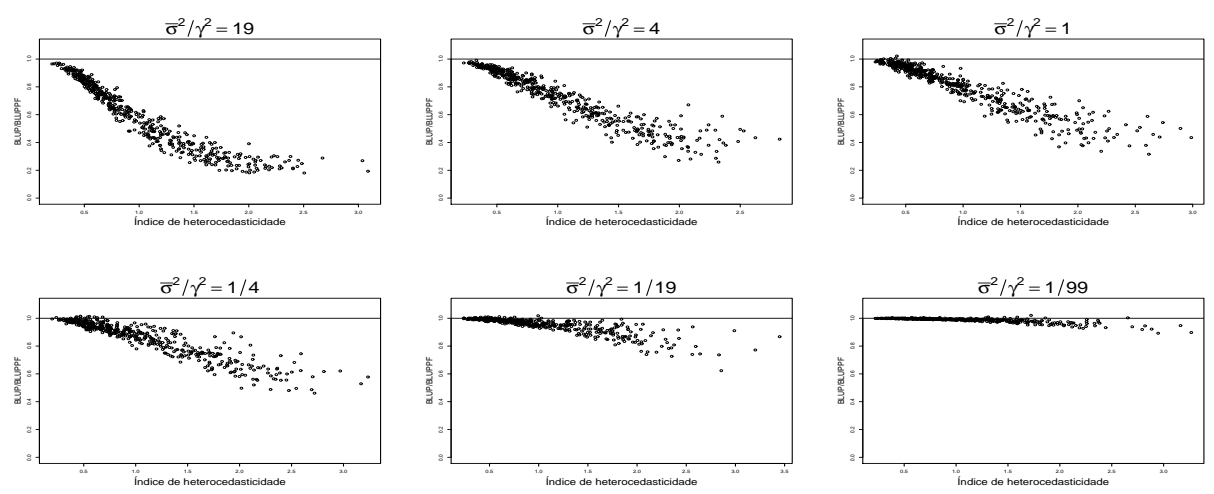

Y's gerados com distribuição assimétrica à esquerda
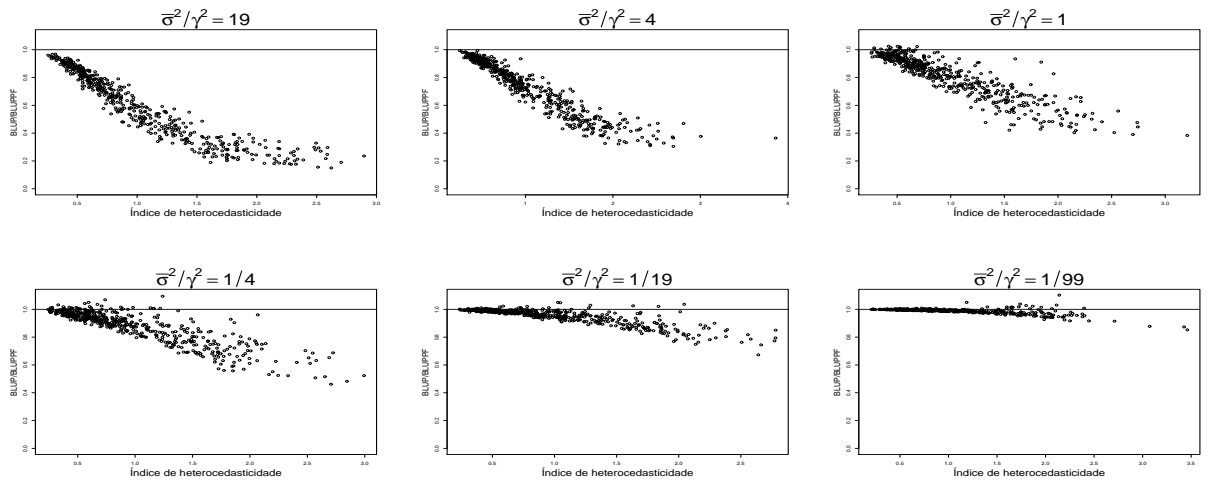

Y's gerados com distribuição assimétrica à direita
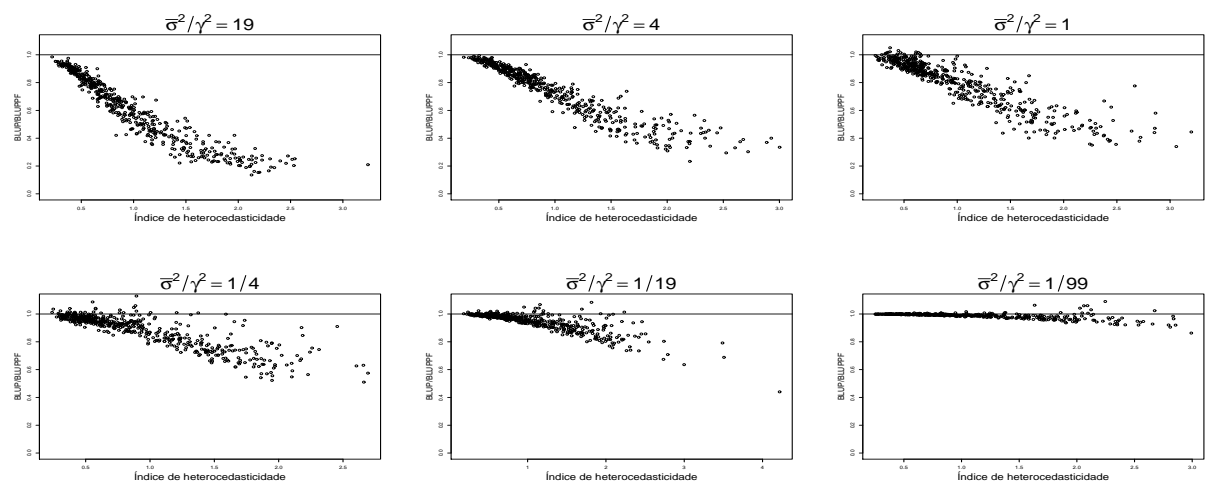
Figura J.3: Quocientes $\operatorname{EQM}\left[\hat{Q}_{i}^{(1)}\right] / \operatorname{EMQ}\left[\hat{Q}_{i}^{(2)}\right]$ sob erros de medida endógenos com distribuição gama, $N=50$ e $n=25$.

Y's gerados com distribuição uniforme
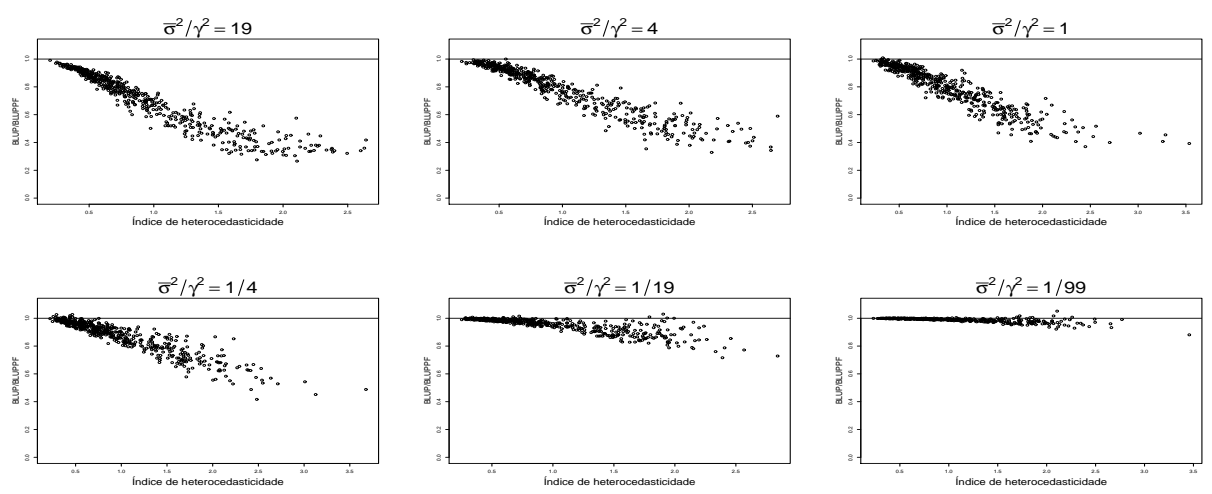

Y's gerados com distribuição assimétrica à esquerda
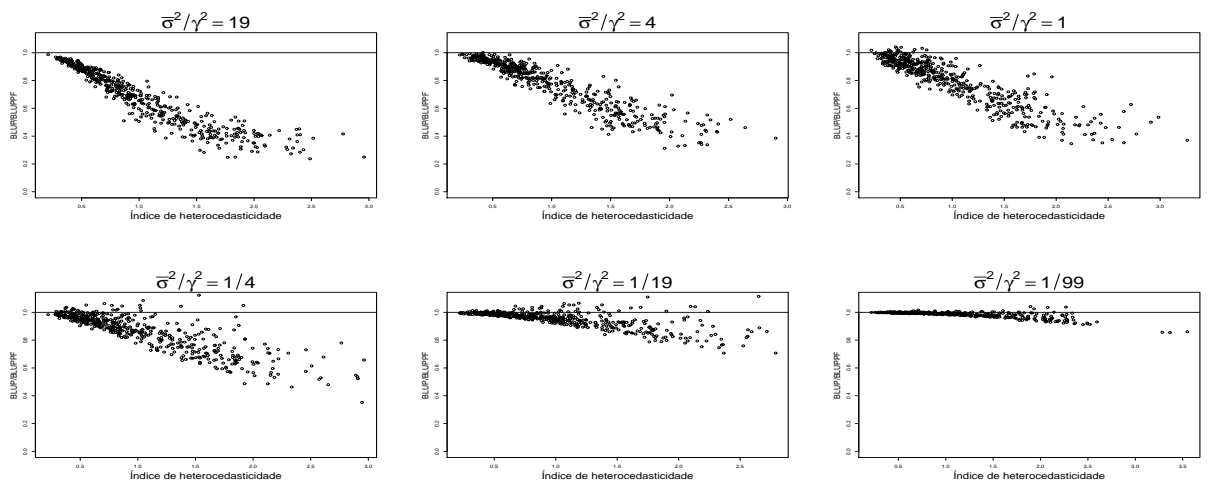

Y's gerados com distribuição assimétrica à direita
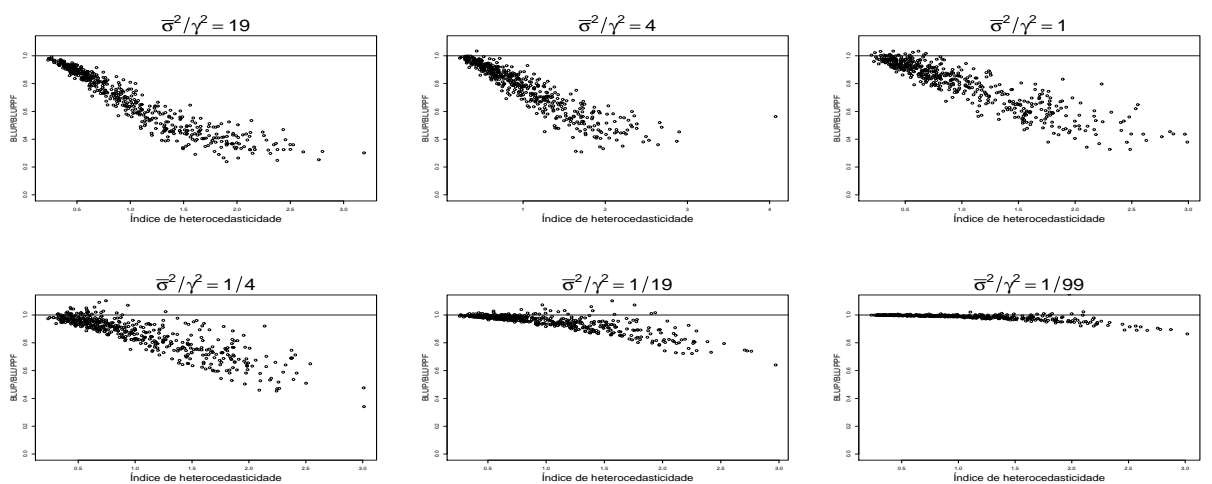
Figura J.4: Quocientes $\operatorname{EQM}\left[\hat{Q}_{i}^{(1)}\right] / \operatorname{EMQ}\left[\hat{Q}_{i}^{(2)}\right]$ sob erros de medida endógenos com distribuição gama, $N=100$ e $n=10$.

Y's gerados com distribuição uniforme
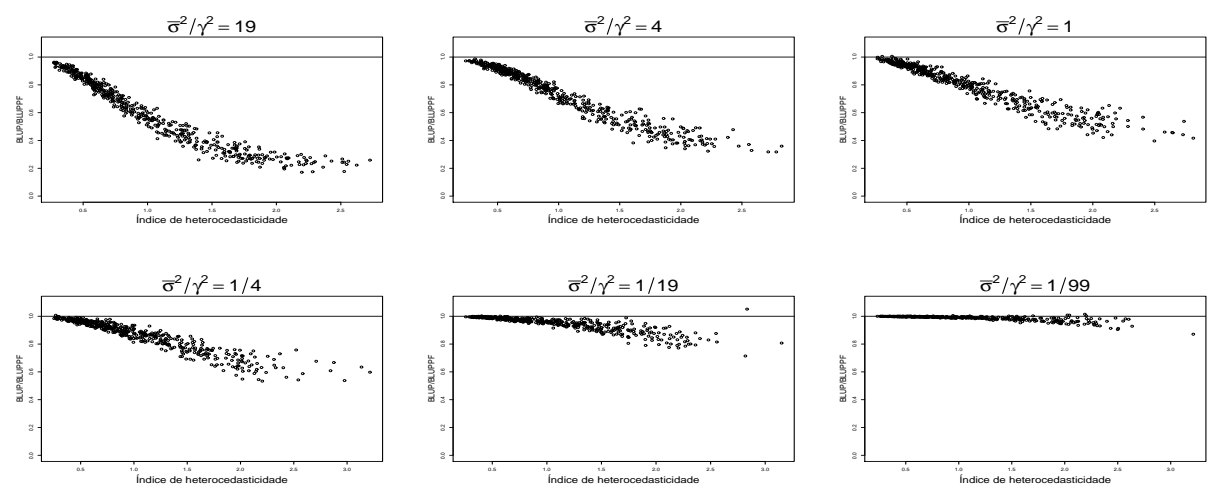

Y's gerados com distribuição assimétrica à esquerda
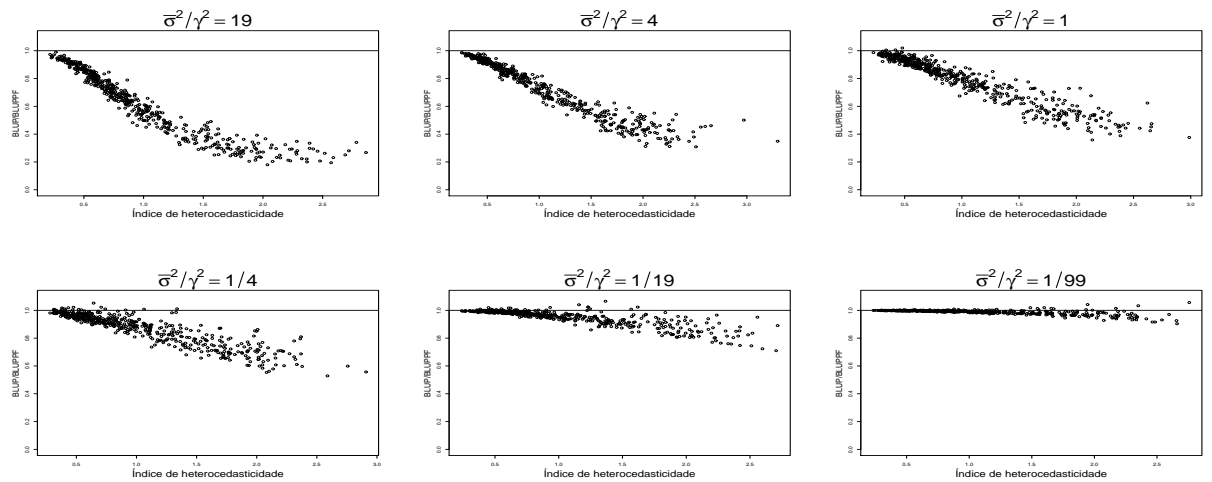

Y's gerados com distribuição assimétrica à direita
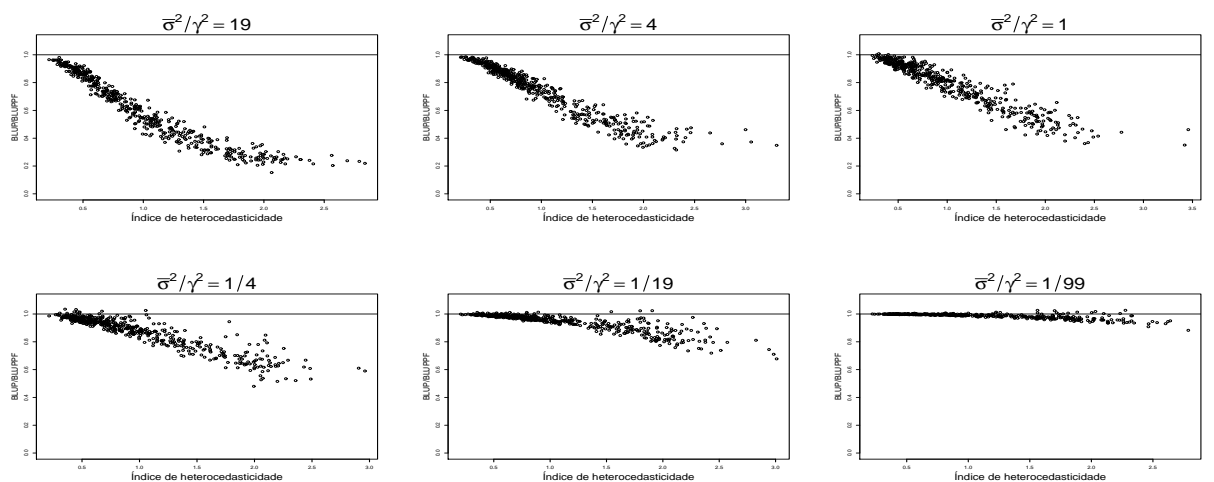
Figura J.5: Quocientes $\operatorname{EQM}\left[\hat{Q}_{i}^{(1)}\right] / \operatorname{EMQ}\left[\hat{Q}_{i}^{(2)}\right]$ sob erros de medida endógenos com distribuição gama, $N=100$ e $n=30$.

Y's gerados com distribuição uniforme
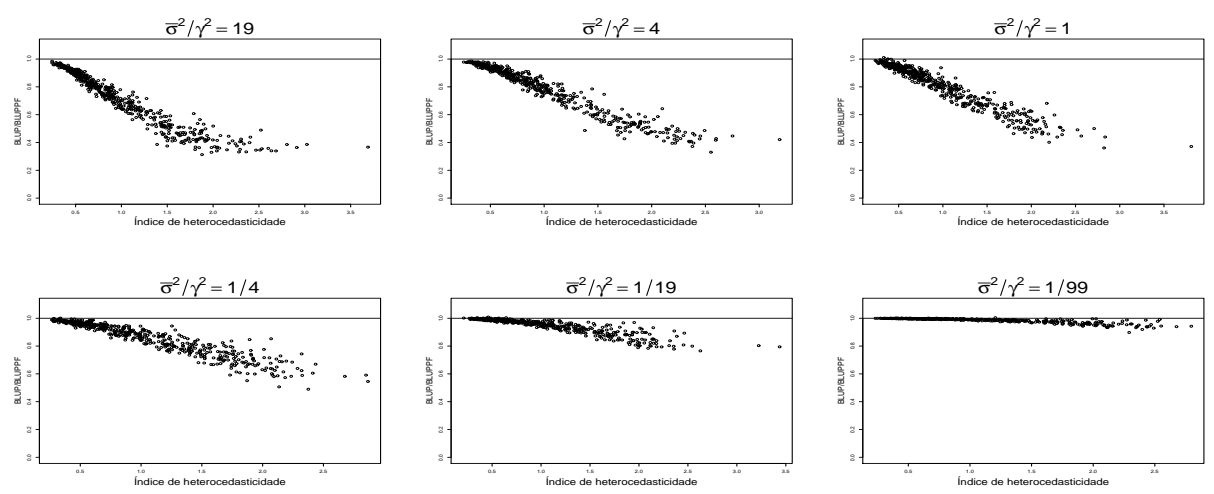

Y's gerados com distribuição assimétrica à esquerda
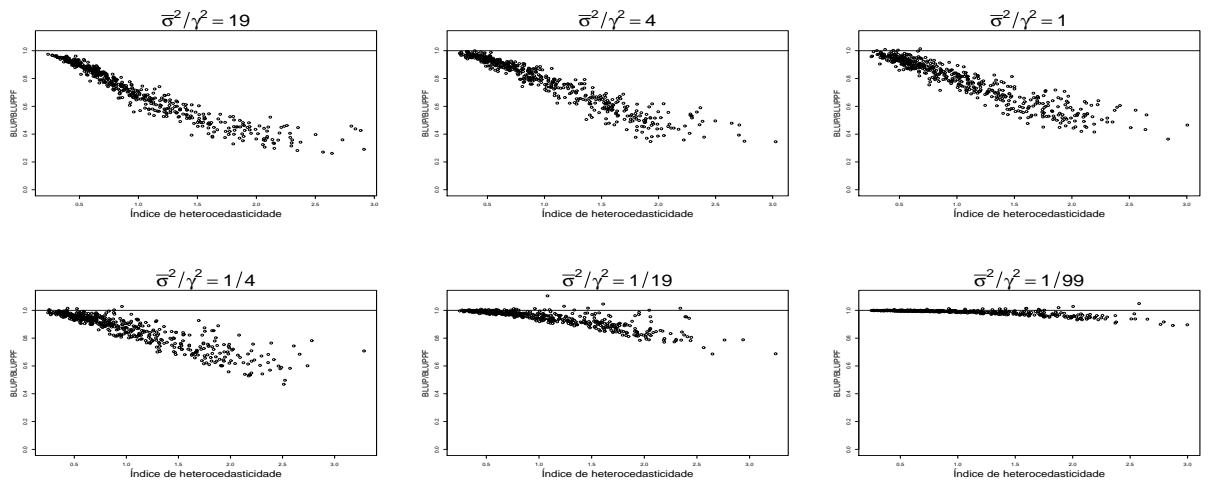

Y's gerados com distribuição assimétrica à direita
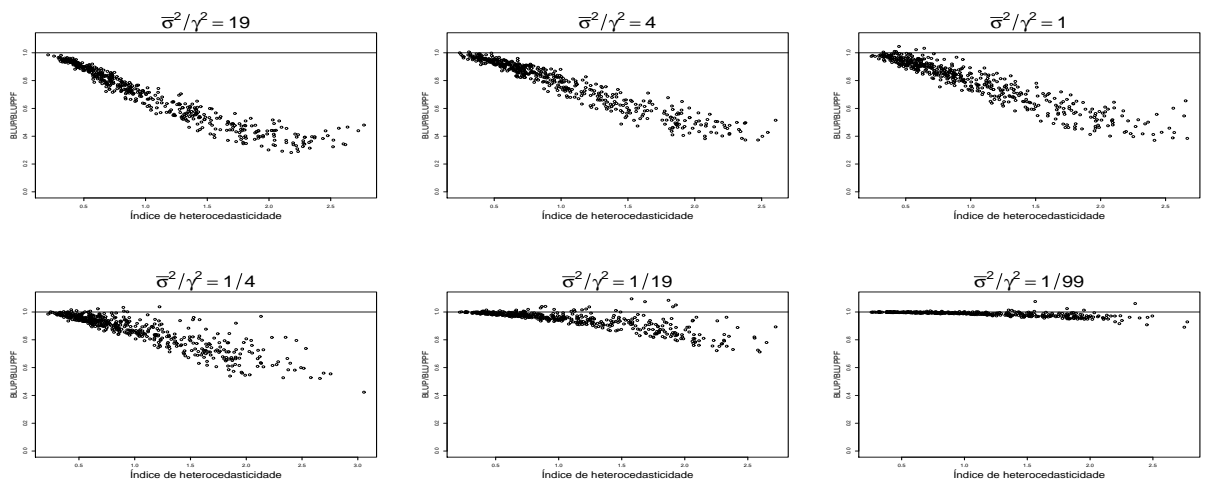
Figura J.6: Quocientes $\operatorname{EQM}\left[\hat{Q}_{i}^{(1)}\right] / \operatorname{EMQ}\left[\hat{Q}_{i}^{(2)}\right]$ sob erros de medida endógenos com distribuição gama, $N=100$ e $n=50$.

Y's gerados com distribuição uniforme
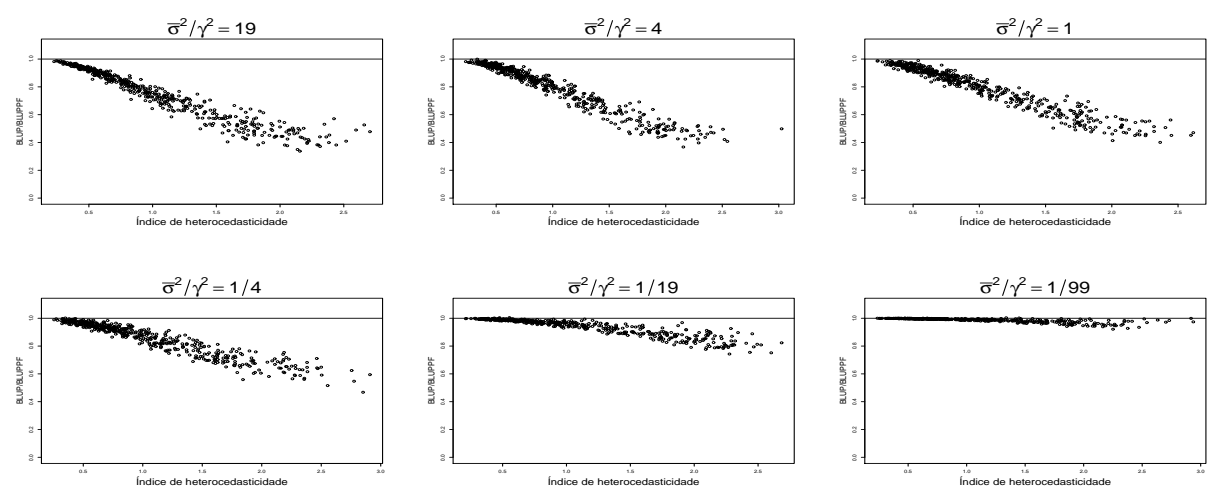

Y's gerados com distribuição assimétrica à esquerda
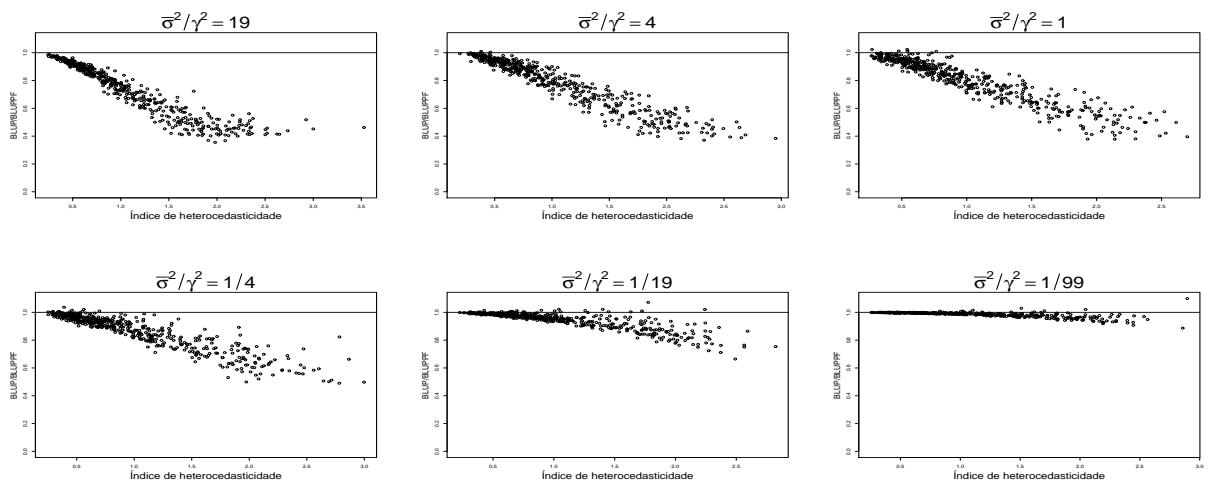

Y's gerados com distribuição assimétrica à direita
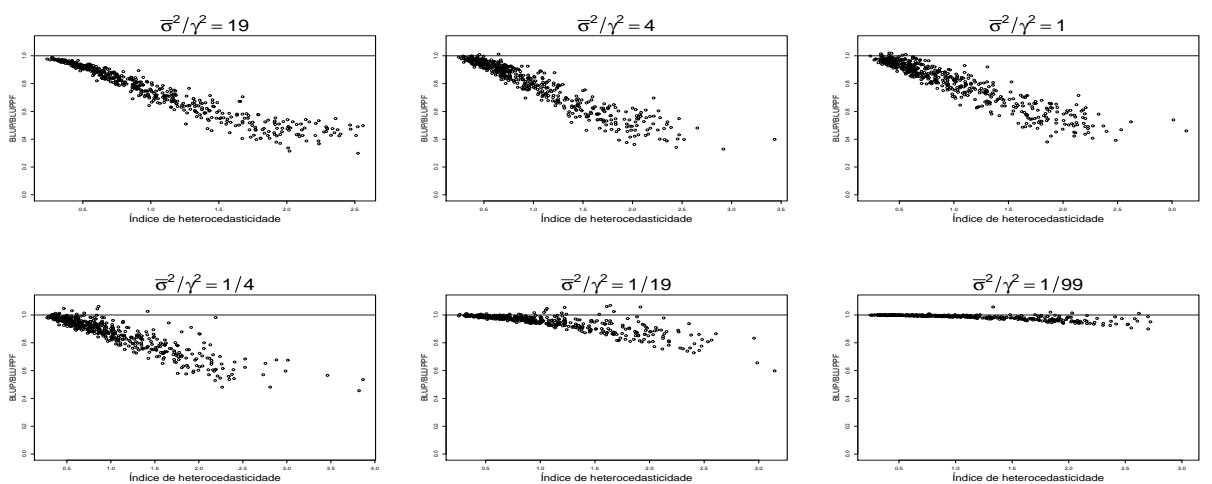
Figura J.7: Quocientes $\operatorname{EQM}\left[\hat{Q}_{i}^{(1)}\right] / \operatorname{EMQ}\left[\hat{Q}_{i}^{(2)}\right]$ sob erros de medida endógenos com distribuição gama, $N=300$ e $n=30$.

Y's gerados com distribuição uniforme
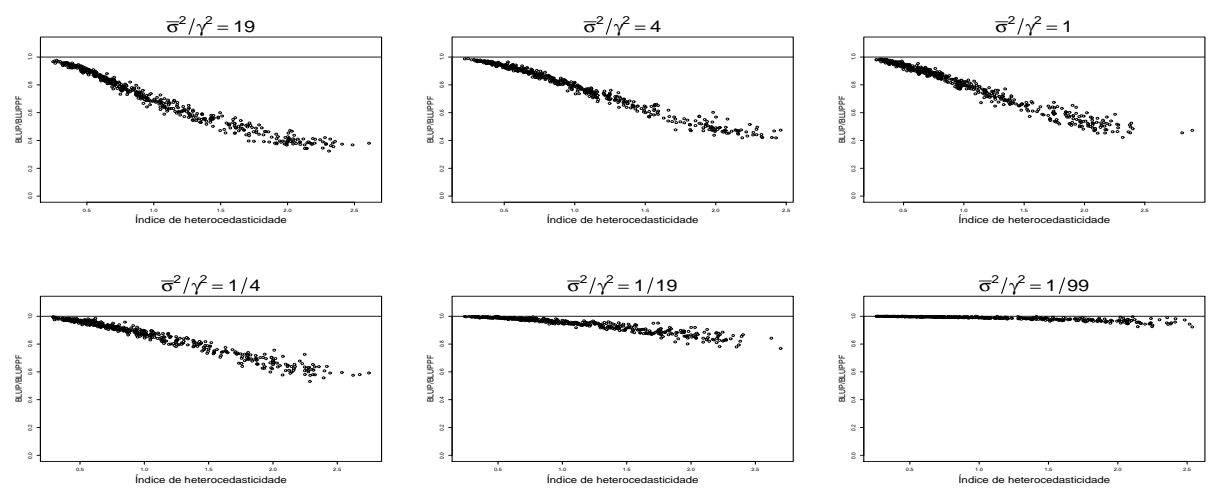

Y's gerados com distribuição assimétrica à esquerda
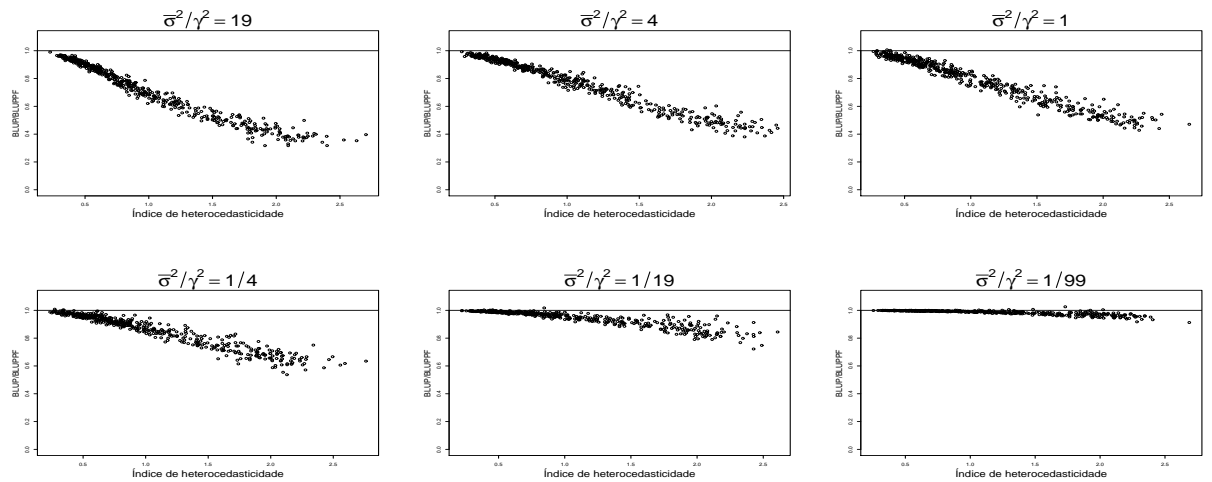

Y's gerados com distribuição assimétrica à direita
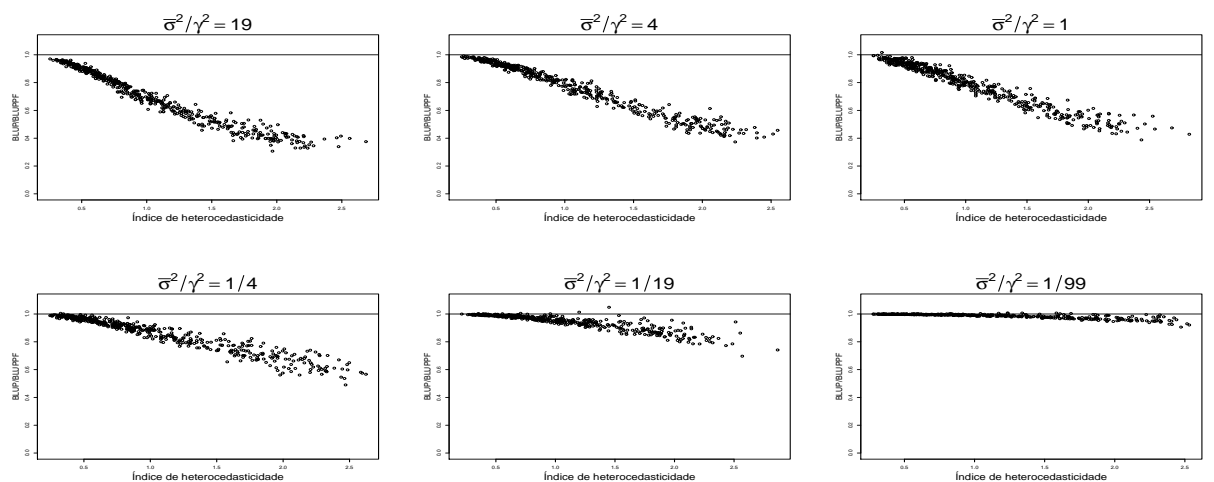
Figura J.8: Quocientes $\operatorname{EQM}\left[\hat{Q}_{i}^{(1)}\right] / \operatorname{EMQ}\left[\hat{Q}_{i}^{(2)}\right]$ sob erros de medida endógenos com distribuição gama, $N=300$ e $n=50$.

Y's gerados com distribuição uniforme
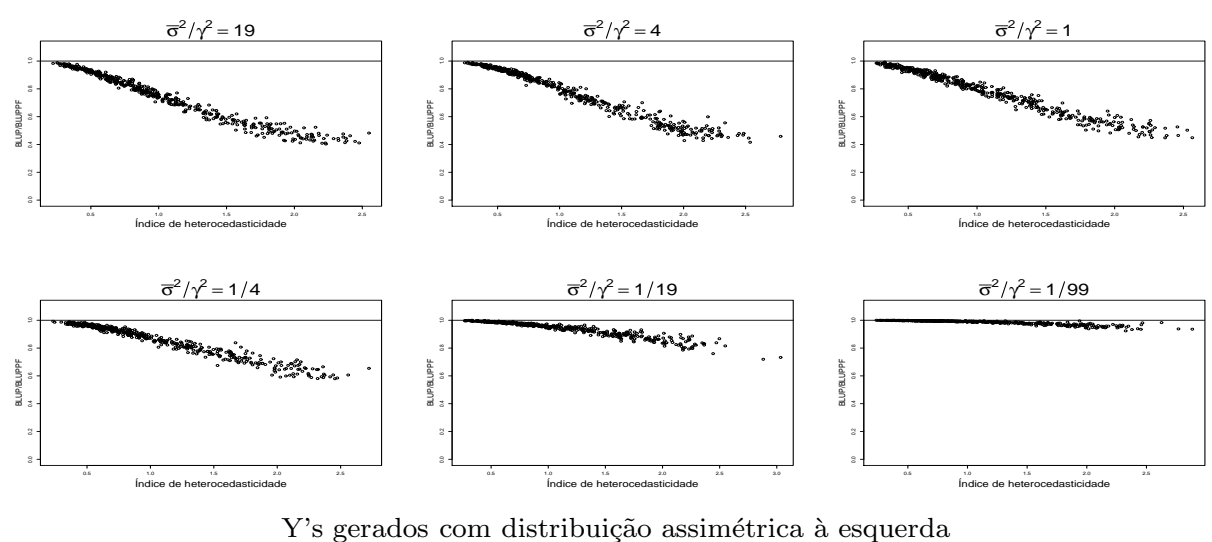

Y's gerados com distribuição assimétrica à esquerda
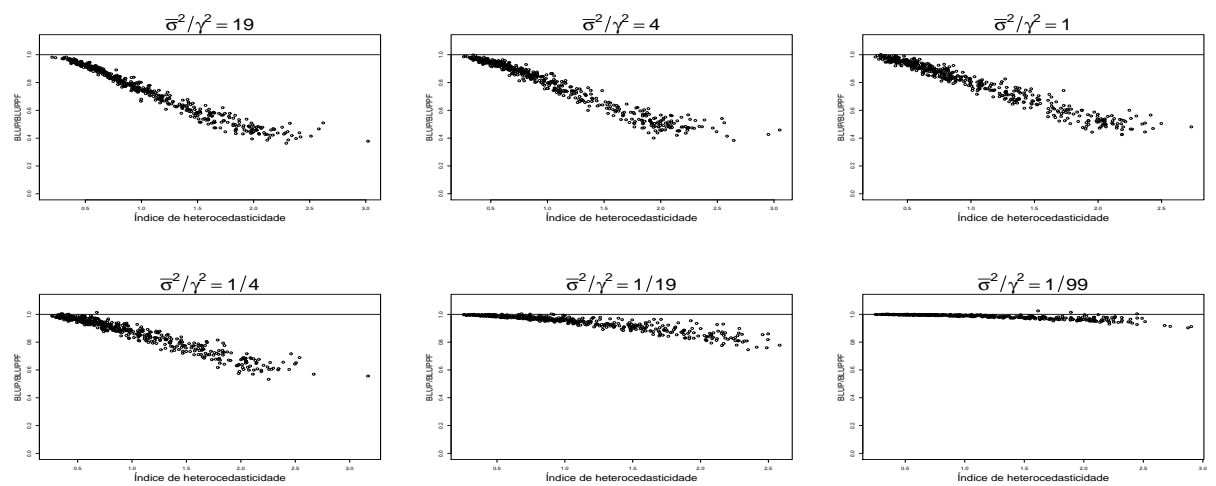

Y's gerados com distribuição assimétrica à direita
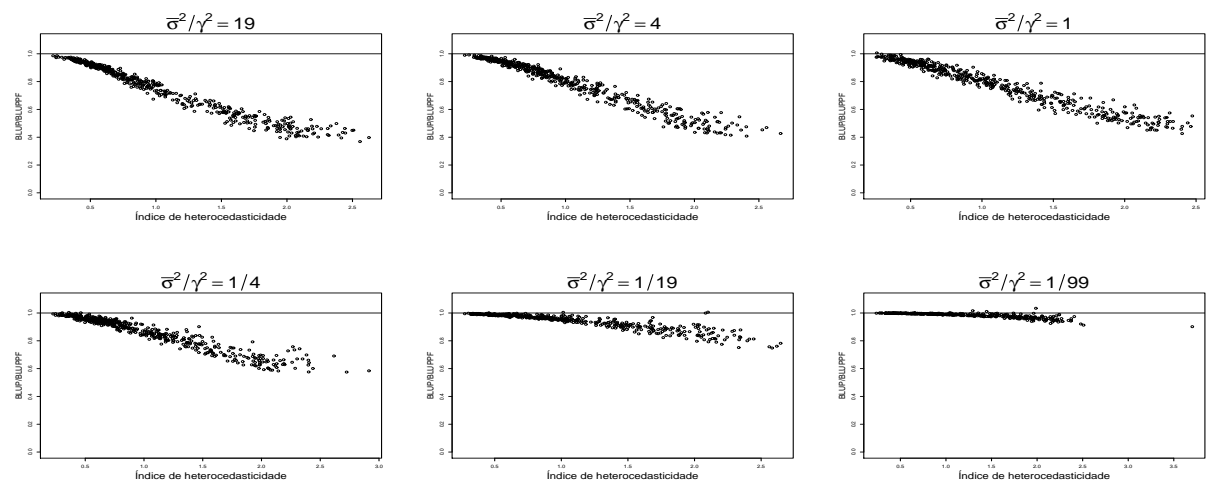
Figura J.9: Quocientes $\operatorname{EQM}\left[\hat{Q}_{i}^{(1)}\right] / \operatorname{EMQ}\left[\hat{Q}_{i}^{(2)}\right]$ sob erros de medida endógenos com distribuição gama, $N=300$ e $n=100$.

Y's gerados com distribuição uniforme
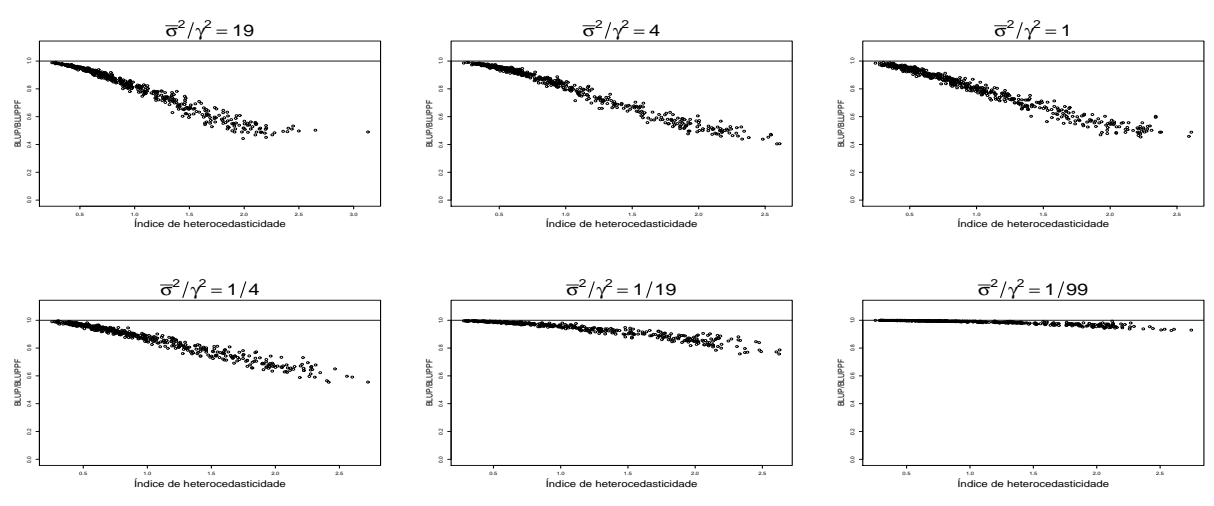

Y's gerados com distribuição assimétrica à esquerda
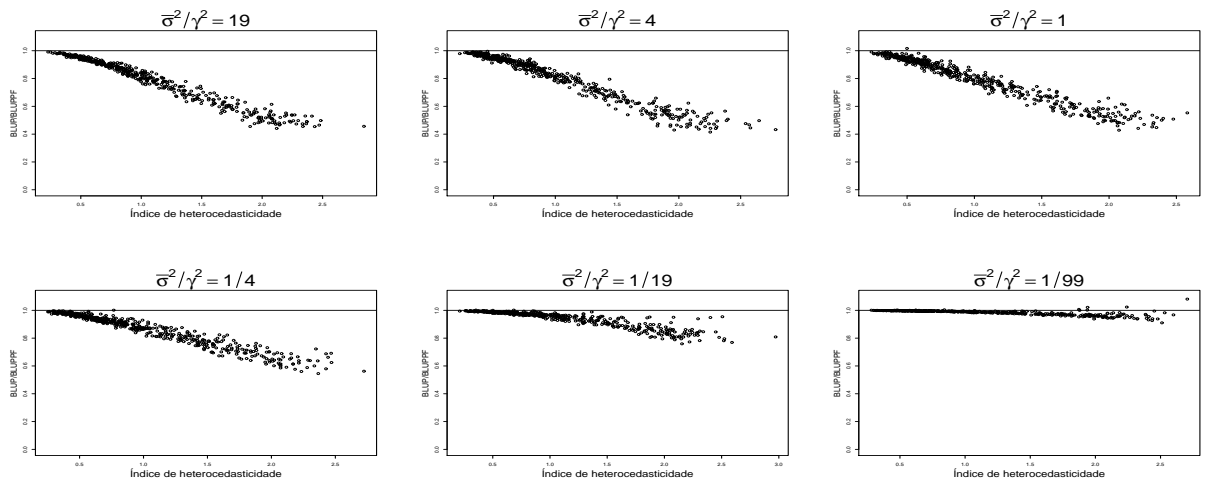

Y's gerados com distribuição assimétrica à direita
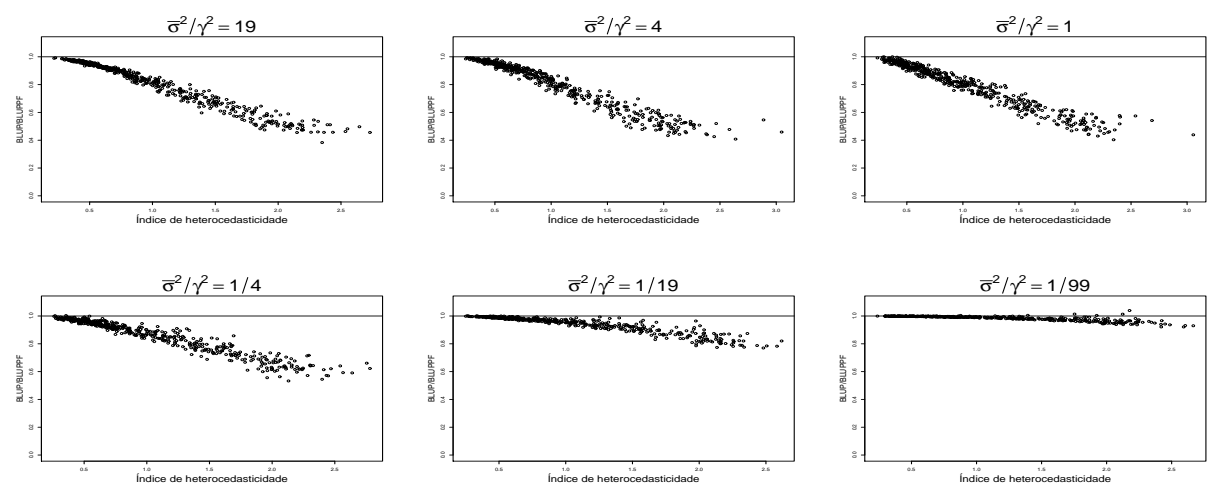


\section{Apêndice $K$}

\section{Códigos R do Capítulo 4}

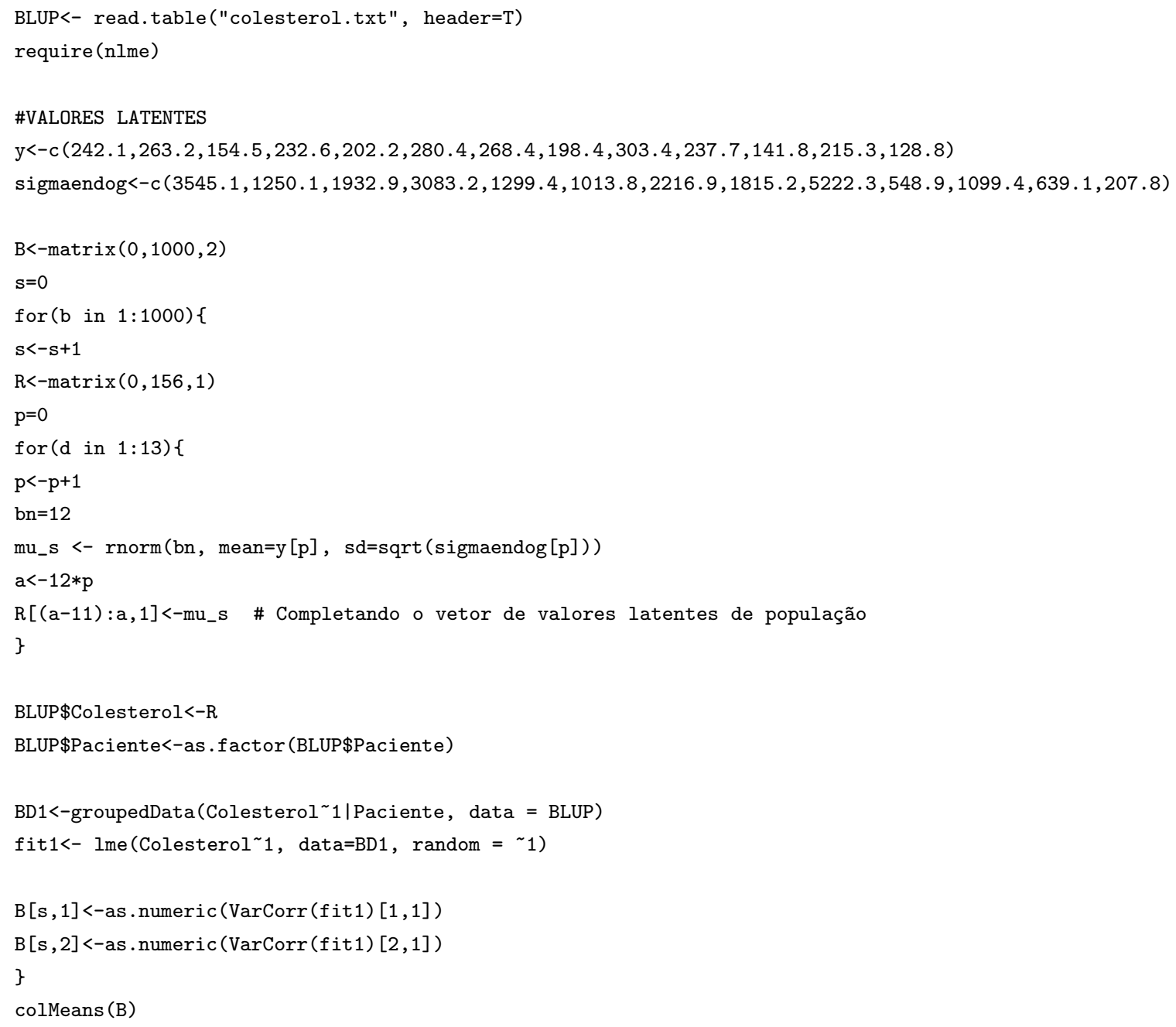




\section{Referências Bibliográficas}

Bolfarine, H. (1991), 'Finite-population prediction under error-in-variables superpopulation models', The Canadian Journal of Statistics 19, 191-207.

Bolfarine, H. \& Zacks, S. (1992), Prediction Theory for Finite Populations, New York: Springer-Verlag.

Bolfarine, H., Zacks, S., Elian, S. \& Rodrigues, J. (1994), 'Optimal prediction of the finite population regression coefficient', Sankhyā B 56, 1-10.

Buonaccorsi, J. (2006), 'Estimation in two-stage models with heteroscedasticity', International Statistical Review 74, 403-418.

Cassel, C. M., Särndal, C. E. \& Wretman, J. H. (1977), Foundations of Inference in Survey Sampling, New York: Wiley.

Cochran, W. G. (1977), Sampling Techniques, New York: Wiley.

Demidenko, E. (2004), Mixed Models: Theory and Applications, New York: Wiley.

Diggle, P., Heagerty, P., Liang, K. \& Zeger, S. (2002), Analysis of Longitudinal Data, New York: Oxford University Press.

Fitzmaurice, G., Davidian, M., Verbeke, G. \& Molenberghs, G. (2008), Longitudinal Data Analysis: A Handbook of Modern Statistical Methods, New York: Chapman \& Hall.

Godambe, V. P. (1955), 'A unified theory of sampling from finite populations', Journal of the Royal Statistical Society, Serie B 17, 269-278.

Goldberger, A. (1962), 'Best linear unbiased prediction in the generalized linear regression model', Journal of the American Statistical Association 57, 369-375.

Harville, D. A. (1997), Matrix Algebra From A Statistician's Perspective, New York: Springer-Verlag.

Henderson, H. V. \& Searle, S. R. (1981), 'On deriving the inverse of a sum of matrices', SIAM Review 23, 53-60.

Horvitz, D. G. \& Thompson, D. J. (1952), 'A generalization of sampling without replacement from a finite universe', Journal of the American Statistical Association 47, 663-685.

Lencina, V. B. (2002), 'Modelos de efeitos aleatórios e populações finitas', Unpublished PhD thesis, Statistics, USP. São Paulo: Universidade de São Paulo . 
Lencina, V. B., Singer, J. M. \& Stanek III, E. J. (2005), 'Much ado about nothing: the mixed models controversy revisited', International Statistical Review 73, 9-20.

McCulloch, C. E. \& Searle, S. R. (2001), Generalized, Linear, and Mixed Model, Wiley, New York.

Merriam, P. A., Ockene, I. S., Hebert, J. R., Rosal, M. C. \& Matthews, C. E. (1999), 'Seasonal variation of blood cholesterol levels: Study methodology', Journal of Biologic Rhythms 14, 330-339.

Neter, J., Kutner, M. H., Nachtsheim, C. J. \& Wasserman, W. (1996), Applied Linear Statistical Models, Boston: WCB/McGraw-Hill.

Rao, J. \& Bellhouse, D. (1978), 'Optimal estimation of a finite population mean under generalized random permutation models', Journal of Statistical Planning and Inference 2, 125-141.

Robinson, G. K. (1991), 'That blup is a good thing: The estimation of random effects', Statistical Science 6, 15-51.

Royall, R. (1968), 'An old approach to finite population sampling theory', Journal of the American Statistical Association 63, 1269-1279.

Royall, R. (1970), 'On finite population sampling theory under certain linear regression models', Biometrika 57, 377387.

Scott, A. J. \& Smith, T. M. F. (1969), 'Estimation in multistage surveys', Journal of the American Statistical Association 64, 830-840.

Searle, S. R. (1982), Matrix Algebra Useful for Statistics, New York: Wiley.

Singer, J. M., Stanek III, E. J., Lencina, V. B., González, L. M., Li, W. \& San Martino, S. (2009), 'Prediction with measurement error: do we really understand the blup?', Submitted .

Smith, T. M. F. (1976), 'The foundations of survey sampling: A review', Journal of the Royal Statistical Society, Serie A 139, 183-204.

Särndal, C. E., Swensson, B. \& Wretman, J. (1992), Model Assisted Survey Sampling, New York: Springer-Verlag.

Stanek III, E. J. \& Singer, J. M. (2004), 'Predicting random effects from finite population clustered samples with response error', Journal of the American Statistical Association 99, 1119-1130.

Stanek III, E. J. \& Singer, J. M. (2008), 'Predicting random effects with an expanded finite population mixed model', Journal of Statistical Planning and Inference 138 (10,1), 2991-3004.

Stanek III, E. J., Well, A. \& Ockene, I. (1999), 'Why not routinely use best linear unbiased predictors (blups) as estimates of cholesterol, per cent fat from kcal and physical activity?', Statistics in Medicine 18, 2943-2959.

Stanek III, E., Singer, J. \& Lencina, V. (2004), 'A unified approach to estimation and predition under simple random sampling', Journal of Statistical Planning and Inference 121, 325-338.

Sukhatme, P. V., e. a. (1984), Sampling Theory of Surveys Applications, lowa State University Press.

Valliant, R., Dorfman, H. A. \& Royall, R. M. (2000), Finite Population Sampling and Inference., New York: Wiley.

Verbeke, G. \& Molenberghs, G. (2000), Linear Mixed Models for Longitudinal Data., New York: Springer-Verlag. 